\title{
Genome-wide screening and genetic networks in pleiotropic drug resistance, oxidative stress, and drug targets in S. cerevisiae
}

\author{
By \\ Ploi Yibmantasiri \\ Victoria University of Wellington \\ A thesis submitted to the Victoria University of Wellington \\ in fulfilment of the requirements for the degree of \\ Doctor of Philosophy \\ in Cell and Molecular Bioscience
}

Victoria University of Wellington

2012 


\section{Abstract}

One of the major problems in biology is to identify genes that are involved in specific processes. Classical genetics and biochemistry, although powerful and informative, can be very labour intensive and do not necessarily characterise networked genes in processes that may overarch numerous biochemical pathways. Here we utilised genomic tools that are capable of defining networks to identify genes involved the complex target mode-of-action of a novel antifungal compound, neothyonidioside and in regulating specific stress processes and the PDR phenotype.

The first part of this study investigated the mode-of-action of the antifungal compound, neothyonidioside (neo). We developed a neo resistant mutant strain then utilising a modification of SGAM, a genetic mapping tool, and application of genome-wide chemical-genetic profiling, we identified the neo resistant locus NCP1. This gene acts at a late step in ergosterol biosynthesis but is not the target of neo. The finding that many of the component genes in the ESCRT complex were necessary for neo resistance allowed us to predict and verify by high-content fluorescence microcopy that interruptions in the endosome-multivesicular body pathway were involved. From the known function of the ESCRT proteins and that neo binds ergosterol only above threshold concentrations of ergosterol (explaining the mutant phenotype) we concluded that neo disruption of membrane curvature and fusion capability in the endosome-vacuole pathway is its target.

In the second part of this study we identified genes in a genome-wide fashion that modulate the pleiotropic drug resistance (PDR) phenotype and oxidative stress response. Many PDR targets are well studied ABC transporters (e.g. PDR5, YOR1), but the modulating events between xenobiotic sensing and transcription factor activation, and possible crosstalk between PDR and other stress responses such as oxidative stress are not well characterised.

To identify specific genes involved in the PDR and oxidative stress processes, we developed a fluorescent reporter screen for effects on the PDR-target ABCtransporters, Pdr5p and Yor1p tagged with GFP. For the oxidative stress response, the oxidative stress (OS) transcription factor Yap1p tagged with GFP was used. Each reporter was placed in the yeast non-essential gene deletion 
background of $\sim 4800$ strains which were then subjected to either xenobiotic treatments (PDR -GFP reporters) or oxidant treatments (Yap1p-GFP). We then screened for gene deletions which prevented the normal upregulation of PDR reporters in the presence of xenobiotics. Controls were included in the screens that assured we were assessing genes that must contribute to or act before the transcription of the $A B C$-transporters. A similar screening strategy was pursued for identifying gene deletions that prevent the normal nuclear re-localisation of Yap1p in the presence of oxidants.

A major finding in this study was identification of genes contributing to the PDR phenotype that involved signalling (Rho-GTPase, MAPK), that were involved in RNA polymerase II mediator complexes and chromatin modification (subunits of ADA and SAGA histone acetyltransferase complexes), and that were involved in sphingo/phosphorlipids biosynthesis. Secondary screens comprising spot dilution growth assays and Western blots of Pdr5p abundance confirmed key genes of the primary screen and showed that these were specific and not global transcriptional effects.For some of the gene-dependencies, our results can only be construed to indicate the existence of alternative pathways underpinning the PDR phenotype in a Pdr1p/Pdr3p independent manner.

We then supposed that if in fact PDR phenotypes are the result of genetic networks, then genes known to interact with the most highly connected hubs from our PDR screen results should also to some extent contribute to the PDR phenotype (spot dilution growth assays, Western blot abundance). A selection of 18 such genes that also appeared in our primary screen but were deemed to be below the cut-off point were phenotype tested and in $60 \%$ of the cases showed similar phenotypes to the genes already identified. This result not only proved the validity of the screening methods but validated the original supposition, i.e. that PDR phenotypes can be affected, through gene networks. 


\section{Acknowledgements}

$\mathrm{PhD}$ research has been a long hard journey and many times the difficulties did not lie in the scientific experiments but the emotional part. How do you cope when experiments fail again and again, how do you stay focussed, and how do you overcome all the stress in the write up state where all you want to do is give up and sleep. Just like cells we have all the mechanisms to cope with stresses, I have many people both academic and at home who have helped me, guided me and supported me through my PhD. To me they all contributed to this work, and without both academic and emotional support from all of them, I would not have been able to start or finish this work.

First and foremost, I would like to THANK (in bold and capital) to my supervisor Professor Paul Atkinson. You are not just a supervisor, you are a great teacher who always always believes and has faith in your students. Who values and treats his students and their ideas with respect and open mind. You taught me to think, to see the big picture, to live, and you had faith in me even when I did not. You taught me all the slangs in my dictionary which I always have and always will use at the most appropriate (inappropriate) moments. The seventh planet in the solar system will always be my favourite. I really do think that our theory about Gas Giants and Klingons and formation of the solar system is the most correct one, and that everything can be explained by putting the word "dark" in front, such as dark matter, dark energy, dark signalling pathway. I am much honoured to be your student. You are one of the "Coolest" men. You always see things beyond what people can see, and I think New Zealand can never keep up with you Paul.

I would also like to thank Dr. David Bellows, my co-supervisor for having me in the lab and making me want to know about drug resistance. Many thanks to Professor John Miller, and Associate Professor Paul Teesdale-Spittle, and Dr. Bill Jordan for all your guidance, ideas, the use of equipments and cookies. Also Dr. David Maass, thank you Dave for being the molecular and PCR god in the lab who makes the most sexist comments and yummiest guacamole on the planet.

Secondly, I would like to thank my lovely boyfriend (flatmate), Reece Dixon who after all I put you through for 3 and a half years; you still stuck with me and believed in me. You always were the greatest emotional support, and understood 
me without having to speak. You were the only one who thought I still look pretty on the last week of thesis write up when I had breakouts, dark circles, very snotty nose, bad cold, and potential to breakdown every 5 minutes. I recommend you should get both your brain and eyes checked.

To Goodie Goodie Yee Syuen Low (future Dr. Yeemy), I will never forget how you always say "I don't have time for this" when we invite you to parties, but you walked in the heavy rain and Wellington breeze (continuous Gusty wind) to get me my favourite Thai food and a DVD so I could stay warm at university and write my thesis. You are the best Yeemy. Thank god we were in the same BIOL 111 tutorial.

To Miss Reemy. You are like my older sister. You are the last person who sends me actual birthday and Christmas cards by post. You always were there to pick me up when I am down, and go out of your way to help me with anything. Thank you very very much. Wellington is not the same since you left for US, and thank you CHEM101 that found me in your lab stream.

To Dr. Arun Kanakkanthara, thank you very much for motivating me through all this tough time of finishing lab work and writing up. I will always remember your quotes "highly dedicated", "guilt", "what is this?", and "from tomorrow on wards". Thank you so much for teaching me Western Blots. I have to admit that you are the Western Blot god. Also thanks to Ariane and Vishy for helping me with Western Blots as well.

To Matthew Baird (Mammu), thank you for giving me ride to the book-binding place to bind my thesis at the early hours in the morning. Thank you Nick (Gunny), for drawing my network diagrams,making me cocktails, stealing my food, and bought me cute Totoro (I am still waiting for the Monolo Blahnik though)

The girl pile:

Thank you Russian (Christina), and Katie for reading and correcting my thesis chapters. Thank you Russ for always be so willingly to help me, both in and outside of the lab stuffs. Thank you for teaching me how to drink Vodka like "The Real Russian", and above all, for waiting with me all night to finish my thesis, and putting up with me screaming. Juliana, the hottest sci-fi geek in the Milky Way galaxy, thank you for your amazing caipirinha night, and how you slowly but surely 
transformed me to a sci-fi geek. Thank you to both Dora and Heather, my past and present running partner. I would be a hippo by now if it were not for you ladies who motivated me (thanks for the baking afterwards too). Dr. Veroo00000000, it was a "tragedy" when you left and now the girl piles have gone to extinction. I do not think a new "girl pile" will ever been the same, and no one can replace you in the shower experience. Thank you for all your support Han Han, the fashionista, who invented the "Hi Five" test to test the shortness of tunic.

To Chemical Genetics Gang:

Thank you Rose, for be the most reliable person in the lab who put up with all our demands and whining. Thank you Wormy, Peter William Bircham for writing all my Acapella scripts (to shut me up.... know), for teaching me how to make YPD+ G418 and tasted it with me to make sure we added glucose. I would like you to know that getting my hair cut short will not make me lose weight and that "porky" is not an adjective any girls would like to hear. However, you are the greatest person to go shopping with. Bedo, you always make me laugh with your dirty jokes. Thank you for always be honest with me, "you look shit today Ploi" was a great joke after 10 hours sleep. Thank you for always helping me in the lab and teaching me all the tricks with Word documents. Namal, it has been later Namal..... that is all I can say.

And, everybody in the lab. Thank you. Thank you. Thank you.

Lastly, I would like to thank my mum and dad who always encouraged me to do whatever I liked and believed in me, and gave me the opportunity to come and study here in New Zealand. Who always said they are proud of me. You have been the great support, and "home" where I can always turn to for wise advice and great comfort. I would also like to thank The Coates family and the Sutton Family for opening and giving me my NZ home and family. To Mr. Greame Sutton, my Kiwi Dad, I wish you were here to see this. You have always been my inspiration to work harder and die trying and NEVER give up. This work is also for you...... 


\section{Table of contents}

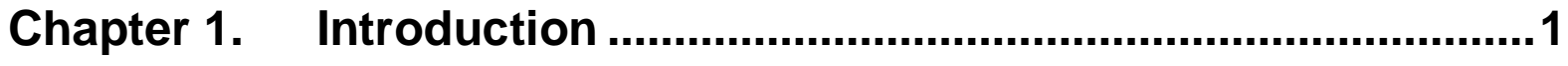

Section I: Genomics as a tool for studying biological processes.................1

Saccharomyces cerevisiae as model organism ...........................................

Section II: Genomics and drug target identification ......................................

Section III: Genomics and multidrug resistance ..........................................10

PDR1 and PDR3; the main transcription factors in PDR ............................. 13

Membrane transporters in pleiotropic drug resistance ..................................17

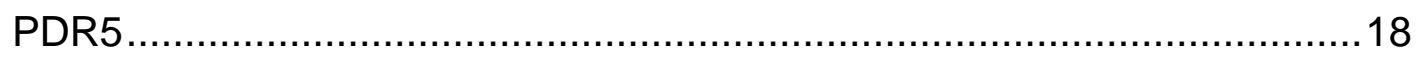

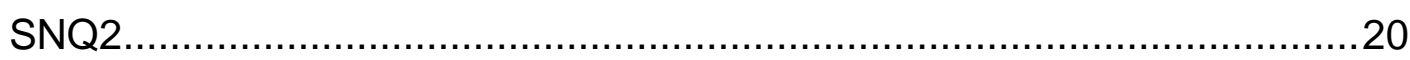

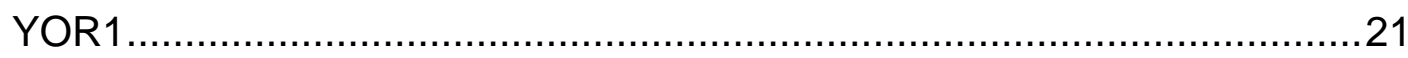

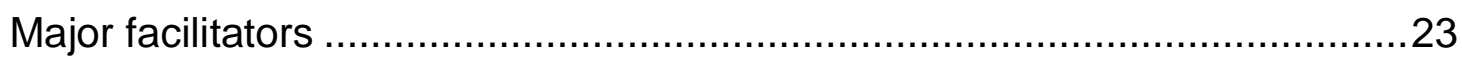

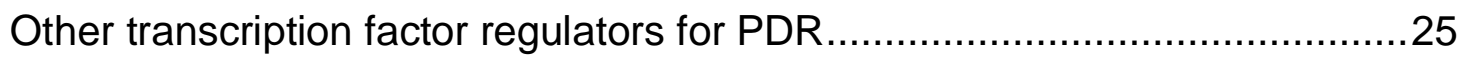

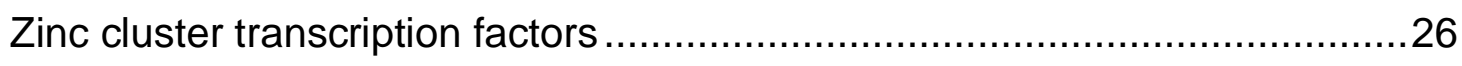

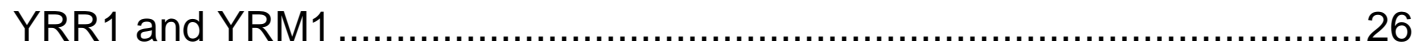

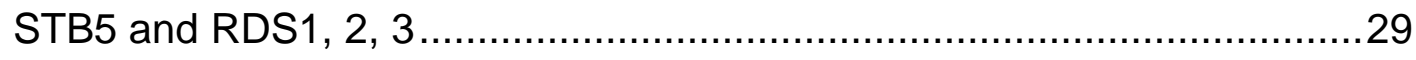

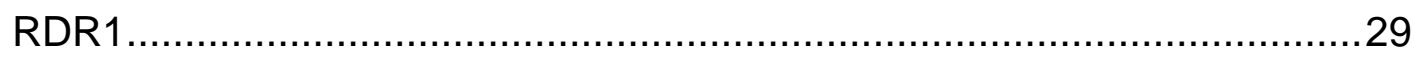

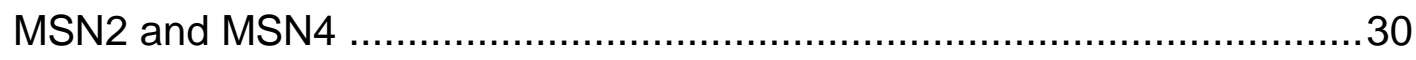

Basic Region-Leucine Zipper Transcriptional regulators (bZIP), Yap1p ..........31

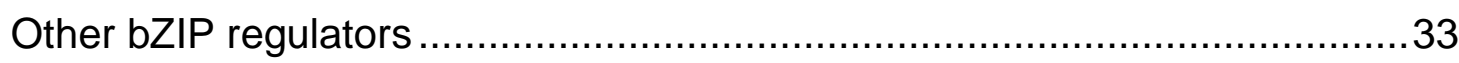

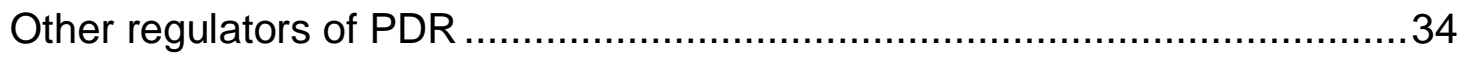

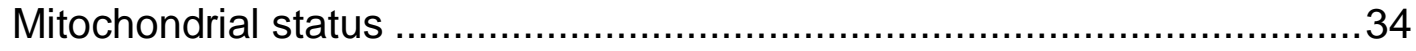

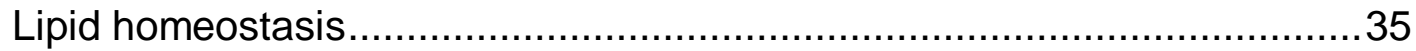

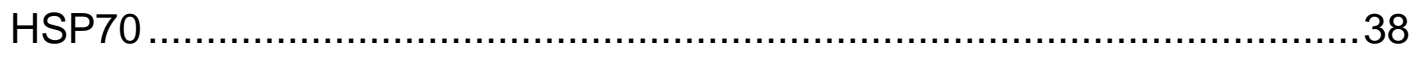

RNA polymerase II mediator complex …….............................................

Mammalian multidrug resistance (MDR) ………..................................... 41

Questions concerning PDR and genomic tools .......................................... 45

Use of a Yap1p-GFP reporter to investigate relationship between oxidative

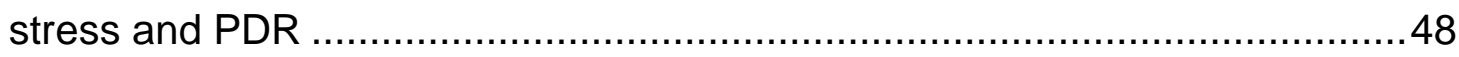

YAP1 and oxidative stress response .................................................. 48 
Mutation in the C-terminus and N-terminus of Yap1p disrupts Yap1p nuclear localisation

Other proteins involved in regulation of Yap1p.......................................51

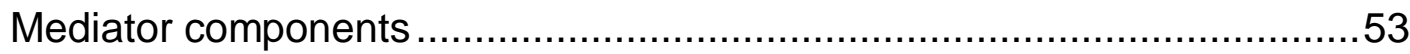

Other transcription factors that regulate oxidative stress response ............53

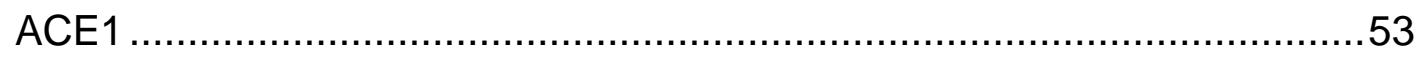

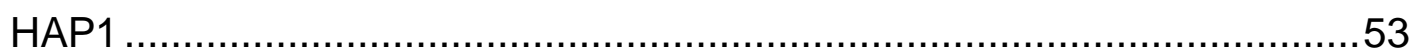

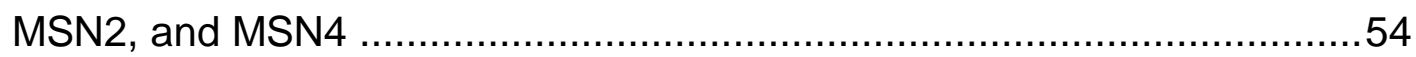

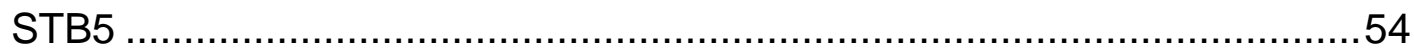

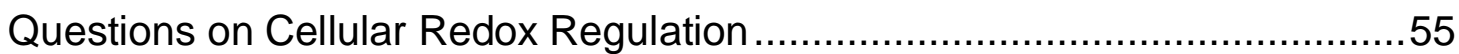

Yap1p and regulation of the multidrug resistance response ..........................56

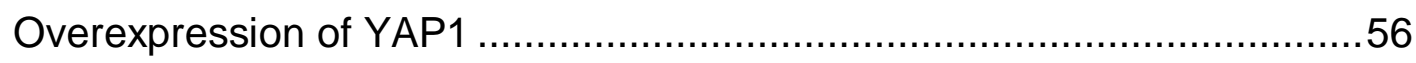

Section IV: Aim, hypothesis and experimental design ................................58

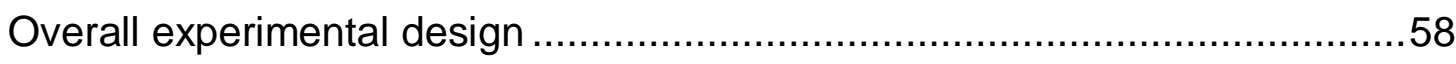

Mode-of-action of toxic compounds using a genome-wide approach for network analysis 58

Identification of upstream regulators of PDR transcription factors

Pdr1p/Pdr3p .60

Identification of regulators/sensors in different stress responses 61

Chapter 2. General Materials and Methods................................63

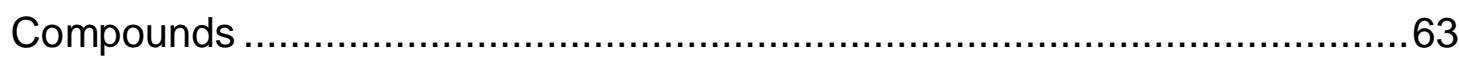

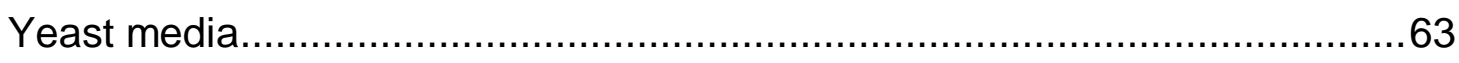

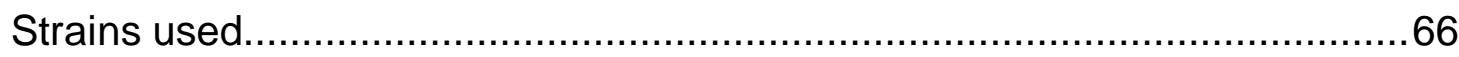

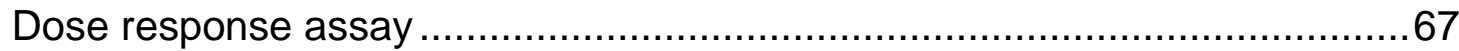

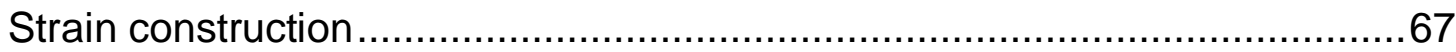

Screening the library of Pharmacological Active Compounds (LOPAC) ..........70

Synthetic Genetic Array (SGA) and drug screening .................................... 71

Identification of possible regulators of pleiotropic drug resistance ..................71

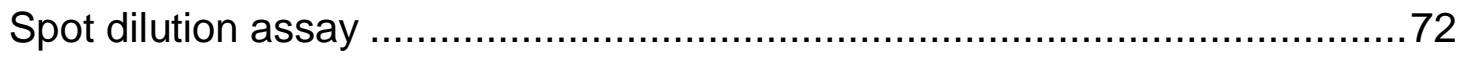

Yeast cell extract and western blot ...................................................... 


\section{Chapter 3. Mechanism of action for a natural product anti- fungal compound, Neothyonidioside, that is not an $A B C$ transporter substrate}

Introduction .74

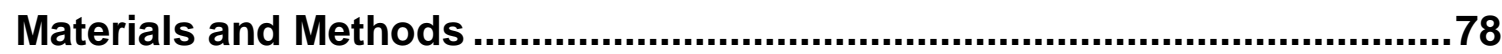

Compound. .78

Identification of neo resistant locus using SGAM and chemical-genetic profiling of neo .78

Vacuole staining and visualisation of live cells .78

Results 79

Neothyonidioside blocks multivesicular body/ vacuole formation. 79

Neothyonidioside treated cells show similar morphology to class B and E vps Mutants.

Neothyonidioside resistant mutant showed that wild type vacuole morphology

Discussion.

Chapter 4. Developing the PDR Response Reporters.

Introduction. .87

Results. 90

Susceptibility of Efflux Pump Reporters .90

Selection of Xenobiotics Which Activate PDR Response .93

Primary Screen Results .96

Validation of the Primary Screen Hits. 100

Genes which Increase Sensitivity in the double mutant $\Delta p d r 1 \Delta p d r 3$ background 104

Gene deletions and effect on Pdr5p abundance. 108

Network analysis predicts gene deletions contribute to PDR phenotype......110

Discussion.

Section I: Methodologies, validation and limitations of the reporter screens.114 (a). Reporters for Investigating Possible PDR Modulators: Primary Screen with Pdr5p and Yor1p Reporters

(b). Does the primary screen specifically identify genes needed for increased expression of $A B C$ transporters in the plasma membrane? .....115 (c). Lack of appearance of putative positive controls 
(d). Validation of primary screen hits using spot dilution growth assays ... 116

(e). Western blot analysis of reporter protein abundance.

Section II: Gene deletions that show negative genetic interaction with

$\Delta \mathrm{pdr} 1 \Delta \mathrm{pdr} 3$

Section III. Signalling pathway related genes that were identified by the screens.

Section IV: Genes involved in RNA-transcription activation (transcription factors, mediator subunits, chromatin modification complexes). 123

Section V: Lipid pathway genes. 126

Section VI: Genes predicted to be involved in the mediation/ modulation of the PDR response via network interaction. 126

Conclusion

\section{Chapter 5. Use of a Yap1p-GFP reporter to investigate relationships between oxidative stress and PDR}

Introduction. 131

Materials and Methods 132

Compounds and Concentration used 132

Yap1p GFP Reporter Strain/ Screens 132

Data analysis. 133

Yap1p-GFP pulse chase experiment.. 134

Results. 135

Yap1p-GFP reporter behaviour in the presence of oxidants 135

Yap1p -GFP reporter peroxide $\left(\mathrm{H}_{2} \mathrm{O}_{2}\right)$ and diethyl maleate screens. 137

Pulse chase experiments of Yap1p-GFP Reporter.

Discussion.

Section I: The validity of the reporter 143

Section II. Yap1p regulation is part of the DNA repair and DNA integrity network.

Section III: A different transcriptional activation complex and different subunits of RNA polymerase II mediator complex are used for Yap1p regulation when exposed to different oxidants.

Section IV: Genes involved in ubiquitination might regulate Yap1p and are more $\mathrm{H}_{2} \mathrm{O}_{2}$ specific

Section V: Stress response genes which are targets of Yap1p might provide a feedback loop for Yap1p regulation. 
Section VI: Yap1p-GFP screens and PDR screens. 149

Conclusion 151

Chapter 6. Final Discussion and Conclusion 153

Section I: New Methodologies 153

(a) Modification of SGAM and application of chemical-genetic profile to identify drug target and mode-of-action 153

(b) Development of genome-wide high content microscopy screen with fluorescent reporters 154

Section II: New gene functions identified in these studies by genomic approaches. 155

(a) Enrichment of ESCRT genes in the non-efflux-pump substrate neothyonidioside 155

(b) Enrichment of genes involved in chromatin modification, RNA polymerase II mediator complexes, and transcription factors in PDR screens. 156

(c) Different genes involved in regulating Yap1p nuclear localization in different oxidants 157

Section III: Predicting and verifying genes from network information .....157

Section IV: New pathways contribute to PDR network. 159

(a) Kinase signalling pathway relay xenobiotics information and activate PDR response. 159

(b) Lipids, mitochondria and PDR. 159

(c) Possible pathways to achieve PDR phenotypes. Lessons from our genomic studies. 160

Section V: Comparison of our results to relevant key papers 162

Section VI: Power of Genomics 163

Future Direction. 164

References. 166

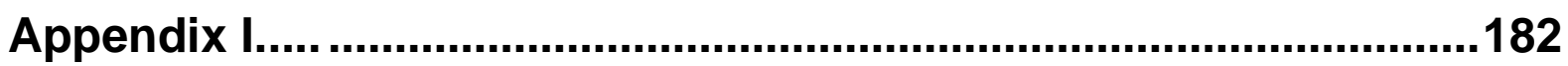

Appendix II. 


\section{Table of Figures}

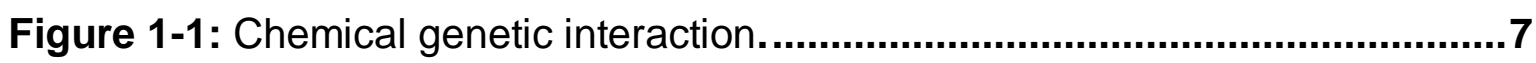

Figure 1-2: Drugs with similar mode-of-action show similar chemical-genetic

profiles.

Figure 1-3: Schematic presentation of RNAseq as technique to identify drug resistant loci .9

Figure 1-4: Mechanisms of multidrug resistance.

Figure 1-5: Possible regulation PDR transcription factors PDR1, PDR3 and YRR1. "Ub" equals sensory input..

Figure 1-6: Membrane $A B C$ transporters in yeast and their locations. .17

Figure 1-7: multidrug $A B C$ transporter structure of Staphylococcus aureus (Sav1866). 18

Figure 1-8: Pdr5p structure. 19

Figure 1-9: Gene regulatory network (GRN) 23

Figure 1-10: PDR regulation in $S$. cerevisiae. 26

Figure 1-11: Overlapping targets between different PDR transcription factors.....28 Figure 1-12: Structure of Basic Leucine Zipper and Zinc Cluster Transcription Factors. 31

Figure 1-13: Yap1p target genes which are involved in PDR phenotypes. 33

Figure 1-14: Composition of eukaryotic plasma membrane 36

Figure 1-15: Yeast mediator complexes.

Figure 1-16: Diagram summarising of the regulators and modulators that contribute to the PDR network and phenotype in yeast.

Figure 1-17: Mechanisms by which cancer cells achieve multidrug resistant phenotype. 44

Figure 1-18: Proposed view of how cells sense xenobiotics 47

Figure 1-19: Different pathways of Oxidative stress response in yeast. 49

Figure 1-20: Model for regulation of Yap1p nuclear localisation. 52

Figure 1-21: Schematic representation of oxidative stress response regulation in yeast 55

Figure 1-22: Structure of known polyene antifungal drugs. 59

Figure 1-23: Schematic presentation of PDR efflux pumps GFP reporters. 61

Figure 1-24: Schematic presentation of Yap1p_GFP reporter 62

Figure 2-1: Tetrad dissection using Singer Dissection Microscope. 
Figure 2-2: Parameter of the Texture Analysis Software......................................70

Figure 3-1: Chemical genetic profile of neothyonidioside.....................................77

Figure 3-2: Plasma membranes and vacuole membranes in wild-type cells untreated and treated with neo was stained with FM 4-64 FX

Figure 3-3: Percentage of cells with wild-type vacuole morphology in control samples and treated samples. 80

Figure 3-4: Vacuole morphology of neo treated cells and vps mutants.

Figure 3-5: Vacuolar morphology of wild-type cells and neothyonidioside resistance mutant cells (neoR).

Figure 3-6: Susceptibility of erg mutants to the tolerance to neothynidioside........83

Figure 4-1: ABC transporter GFP reporters.....................................................91

Figure 4-2: Susceptibility of ABC-GFP reporters...........................................92

Figure 4-3: Fold change GFP reporters in different xenobiotic treatments (control/treated)

Figure 4-4: PDR Primary screen network.

Figure 4-5: Examples of gene deletions that prevented normal upregulation of Pdr5p-GFP and Yor1p-GFP reporters.

Figure 4-6: Examples of gene deletions from primary screens that conferred sensitivity to compound(s) tested.

Figure 4-7: Examples of gene deletions which result in an increase in sensitivity toward compound(s) tested as both single deletions $(\Delta x x x)$ and in the $\Delta p d r 1 \Delta p d r 3$ background ( $\Delta x x x \Delta p d r 1 \Delta p d r 3)$.

Figure 4-8: Example of gene deletions which result in increase in sensitivity toward compound(s) tested only when in $\Delta p d r 1 \Delta p d r 3$ background. 107

Figure 4-9: Network of genes from PDR secondary screen...............................107

Figure 4-10: Gene deletions that reduce Pdr5p abundance...............................109

Figure 4-11: Published genetic (http://thebiogrid.org/) networks among our screen results and some of the known PDR-contributing genes.

Figure 4-12: Published physical interaction (http://thebiogrid.org/) networks among our screen results and some of the known PDR-contributing genes.

Figure 4-13: Signalling genes in the network of genes for PDR secondary validation

Figure 4-14: transcriptional controlling genes in the network of genes for PDR secondary validation 
Figure 4-15: Spatial assembly of the transcription machinery

Figure 4-16: Possible alternative pathways to activate the PDR response in S. cerevisiae.

Figure 5-1: Yap1p-GFP reporter treated with different oxidants. 136

Figure 5-2: The punctate pattern of Yap1p-GFP observed when cells were treated with diamide or diethyl maleate. .137

Figure 5-3 : Examples of gene deletions that prevented normal nuclear localisation of Yap1p-GFP upon treatment with $1 \mathrm{mM} \mathrm{H}_{2} \mathrm{O}_{2}$. 138

Figure 5-4 : Examples of gene deletions that prevented normal nuclear localisation of Yap1p-GFP upon treatment with $2 \mathrm{mM}$ diethyl maleate. 138

Figure 5-5: Gene deletions that prevent normal nuclear localisation of Yap1pGFP upon $\mathrm{H}_{2} \mathrm{O}_{2}$ treatment

Figure 5-6: Gene deletions that prevent normal nuclear localisation of Yap1pGFP upon diethyl maleate treatment 139

Figure 5-7 : Pulse chase experiment of Yap1p-GFP treated with $\mathrm{H}_{2} \mathrm{O}_{2}$ 142

Figure 5-8: The diagram shows that different sets of genes may be used to regulate the Yap1p response to different oxidative stress-inducing agents. 152 Figure 6-1: Diagram representing possible pathways in S.cerevisiae activated by xenobiotics 162 


\section{Table of Tables}

Table 2-1: Compounds used in spot dilution assay ..............................................63

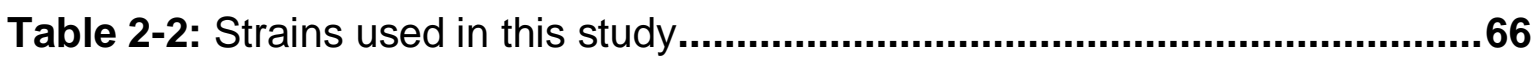

Table 4-1: Compounds from LOPAC Library which upregulated ABC pumps with C-terminal GFP tagged reporters

Table 4-2: Eleven Genes relating to RNA transcription were GO enriched as revealed in the PDR primary screens.

Table 4-3: Results from Validation of Primary Screen Result with Spot Dilution Assays and Western Blots

Table 4-4 : Genes which interact with secondary hits nodes and /or PDR1 PDR3 and might have effect on PDR phenotype

Table 4-5: LOPAC compounds that activate PDR reporters and inhibit signallingrelated pathways or molecules

Table 5-1 :GO function enrichment of gene deletions preventing normal nuclear localisation of Yap1p-GFP reporter upon $\mathrm{H}_{2} \mathrm{O}_{2}$ treatment

Table 5-2 :GO function enrichment of gene deletions preventing normal nuclear localisation of Yap1p-GFP reporter upon diethyl maleate treatment.

Table A-1:List of gene deletions which cause insignificant change in PDR reporters upon atorvastatin treatment 182

Table A-2:Hit genes from Yap1p-GFP screen with $\mathrm{H}_{2} \mathrm{O}_{2}$ treatment. 188

Table A-3 :Hit genes from Yap1p-GFP screen with diethyl maleate treatment...195 


\section{Important genes in this study}

PDR1: Zinc finger transcription factor that regulates the pleiotropic drug response.

PDR3: Transcriptional activator of the pleiotropic drug resistance network. Pdr3p forms heterodimers with Pdr1p.

PDR5: A major plasma membrane $\mathrm{ABC}$ multidrug transporter actively regulated by Pdr1p. Used here as a reporter for Pdr1p and Pdr3p activation.

SNQ2: Plasma membrane $\mathrm{ABC}$ multidrug transporter involved in multidrug resistance and resistance to singlet oxygen species

YOR1: Plasma membrane $A B C$ multidrug transporter. Used here as a reporter for Pdr1p and Pdr3p activation.

YAP1: Basic leucine zipper (bZIP) transcription factor; required for oxidative stress tolerance. Overexpression confers resistance to many compounds, including cycloheximide and fluconazole. Thought to played part in multidrug resistance.

\section{Abbreviations}

ABC transporter: ATP-binding cassette $(\mathrm{ABC})$ transporter

EMS: Ethyl methanesulfonate (act a mutagen)

GSH: Glutathione

ORF: Open reading frame

PKC: Protein kinase C

MAPK: Mitogen-activated protein (MAP) kinases

NLS: Nuclear localising signal

ROS: Reactive oxygen species

SAGA: Histone acetyltransferase complex that contains Spt8p 
SWI/SNF: (SWItch/Sucrose NonFermentable), a nucleosome remodeling complex

TF: Transcription factor

\section{Glossary}

Xenobiotics: toxic or inhibitory small molecules

PDR: Pleiotropic drug resistance, here referred to as mechanisms that cells use to compensate for compound toxicity (such as alternative pathways to the one that has been affected by xenobiotics), as well as classic efflux pumps controlled by Pdr1p/Pdr3p, and other stress responses which can lead to resistance to a variety of compounds

Genetic interaction: "Two genes A and B "genetically interact" when the phenotype generated as the result of mutations in both genes (double mutant ab) is unexpectedly not just an additive phenotype of the two single mutants a and b". (Eddy S. R., 2006 Genetics)

Different forms of genetic interaction are listed below.

Dosage lethality: Form of genetic interaction, overexpression or increased dosage of one gene causes lethality in a strain that is mutated or deleted for another gene.

Dosage rescue: Form of genetic interaction. Dosage-rescue-genetic interaction is inferred when overexpression or increased dosage of one gene rescues the lethality or growth defect of a strain that is mutated or deleted for another gene.

Negative genetic: Form of genetic interaction. This occurs when mutations/deletions in separate genes, each of which alone causes a minimal phenotype, result in a more severe fitness defect or lethality under a given condition when combined in the same cell.

Phenotypic enhancement: In this type of interaction, a genetic interaction is inferred when mutation or overexpression of one gene results in enhancement of 


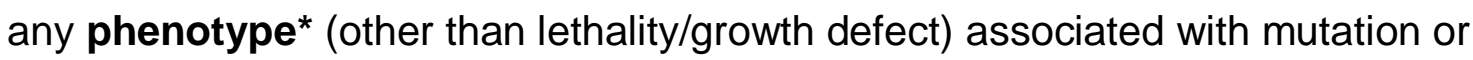
overexpression of another gene.

(* phenotype: observable traits of strains that carry a mutation at that locus)

Phenotypic suppression: In this process, a genetic interaction is inferred when mutation or overexpression of one gene results in suppression of any phenotype (other than lethality/growth defect) associated with mutation or overexpression of another gene.

Positive genetic: Form of genetic interaction. In this type of interaction, mutations/deletions in separate genes, each of which alone cause a minimal phenotype, result in a less severe fitness defect than expected under a given condition when combined in the same cell.

Synthetic genetic interaction: A genetic interaction in which a combination of mutations in two or more genes of a single strain results in a phenotype that is different in degree or nature from the phenotypes conferred by the individual mutations. The most common type of synthetic interaction is synthetic lethality, in which two mutations, neither of which causes inviability when present individually, cause inviability when present together in the same strain. Synthetic interactions may result in other phenotypes, e.g., slow growth or respiratory growth defects. Synthetic interaction can be an indication that the genes involved participate in the same pathway or process; synthetic interaction between two point mutant alleles may be an indication that the gene products physically interact.

Synthetic growth defect: a genetic interaction is inferred when mutations in separate genes, each of which alone cause a minimal phenotype, result in a significant growth defect under a given condition when combined in the same cell.

Synthetic lethality: Form of genetic interaction when mutations or deletions in separate genes, each of which alone cause a minimal phenotype, result in lethality when combined in the same cell under a given condition.

Synthetic rescue: Form of genetic interaction when mutation/deletion of one gene rescues the lethality or growth defect of a mutation in another gene.

Epistasis: a general term for synthetic genetic interactions 


\section{Chapter 1. Introduction}

\section{Section I: Genomics as a tool for studying biological processes}

Much of our understanding of biological processes comes from studying the effects of gene mutation/disruption on the phenotypes of interests. Forward classic genetics utilises mutagens such as ethyl methane sulfonate (EMS), UV, viruses to introduce mutations, then screens for the phenotypes. This method normally produces clearly defined and easy to score phenotypes, and an example of successful application of this approach is the discovery of the major components of the Ras signalling pathways (Boutros and Ahringer 2008). Nevertheless, the identity of the mutations needs to be established, usually through cloning which is labour intensive and time consuming. An alternative is presented by genomic tools, such as the complete sequence and gene deletion sets in model organisms such as the yeast Saccharomyces cerevisiae, the nematode Caenorhabditis elegans, and the fruit fly Drosophila melanogaster. These provide geneticists with powerful means to study gene function in a systematic and genome-wide manner. These collections of genome-wide screening tools, particularly in yeast, have many advantages over the classic genetic approach. Firstly, every gene in the deletion collections has been sequenced and identified, and therefore the resultant phenotype can be recorded for every gene. Secondly, the function of some genes may not be probed in classical genetics, an approach which screens random mutations. In genome-wide screens, the mutations and screen is systematic, and furthermore, all of the genes in the genome are tested simultaneously. In such a systematic process, therefore, "there is no place to hide", and the result is a more complete picture of the genetic contribution to the biological phenotypes and or processes of interest (Carpenter and Sabatini 2004). It has long been known in classical genetics that most phenotypes are the result of complex gene expression (Lobo 2008)_for example, drug resistance (Perlstein, Ruderfer et al. 2007). Yet, classical genetics has not cracked the molecular basis of complex gene expression beyond quantitative trait loci approaches, which are powerful, but limited in their resolution for determining all the underlying genetic contribution. This situation has become a key driver in specific literature of the last decade to utilise genomic tools in a different approach to genetic complexity. 
Biological processes that have been studied using genome-wide screens included, for example, RNA interference (RNAi) screens in order to identify enzymes involved in a development defect in $C$. elegans, and eye development in $D$. melanogaster. Employing various reporter genes in combination with an RNAi (gene knockdown) collection has been used to study the Hedgehog signalling pathway (Carpenter and Sabatini 2004). The reporter genes have allowed examination of intracellular phenotypes, which cannot be seen in gross cellular morphology, growth or in changes in gene expression.

Gene deletion collections (S. cerevisiae) or knockdown collections (C. elegans) have been used for exploring all the genes involved in a growth phenotype in response to drug (Simon and Bedalov 2004; Artal-Sanz, de Jong et al. 2006; Kwok, Ricker et al. 2006), and more recently, phenotypic assays that involve the morphology of cells utilising high-throughput screening microscopes have been developed (Zemanová, Schenk et al. 2003; Pepperkok and Ellenberg 2006; Paran, llan et al. 2007). This has allowed for observation of developmental morphology, and intracellular localization in C. elegans (Chung, Crane et al. 2008) and intracellular localization in S. cerevisiae (Vizeacoumar, van Dyk et al. 2010), as well as for investigation of complexes that are involved in protein folding in ER in S. cerevisiae (Carpenter and Sabatini 2004; Boutros and Ahringer 2008)

A DNA microarray is a collection of gene-specific oligonucleotides that are attached to a solid surface such as a glass slide. Genomic DNA, or complementary DNA from cells subjected to different treatments, or from different tissue types, are hybridised to the slide, and the expression profile on the arrays is analysed. This method is widely used to study processes such as the dynamics of gene expression during development and differentiation, differential expression between normal cells and cancer cells, or gene expression and transcription activity in different stress environments (Causton, Ren et al. 2001; Kurimoto and Saitou 2010).

Although genome-wide based "reagents" (that is deletion strains) are available in a number of genetic model organisms (there is a complete set for Saccharomyces cerevisiae, Saccharomyces pombe, a partial set for C. elegans, and 
approximately 8000 mouse strains as cell lines), the most widely used collection is the yeast deletion array in S. cerevisiae.

\section{Saccharomyces cerevisiae as model organism}

Yeast, especially $S$. cerevisiae or baker's yeast, is a popular model organism, owing to a variety of attributes. These include a high homology to mammalian genes $(31 \%$ of protein coding gene/ORF in yeast have homologues in mammals (Botstein, Chervitz et al. 1997)), their small size, a sequenced compact genome, high rates of homologous recombination, which allows for precise allele-specific genetic manipulation, and existence of both haploid and diploid life cycles. The haploid life cycle allows for easy recovery of recessive mutations and attenuates the necessity of considering epistatic interaction between alleles (Forsburg 2001) by removing dominant epistasis.

S. cerevisiae was the first eukaryote for which precise gene deletion collections were compiled. In yeast, there are approximately 6300 genes that have been identified, and approximately 4800 genes can be deleted without showing growth phenotypic defects when grown in rich medium. This set of non-essential gene "knockouts" has proved to be a valuable tool for studies of many biological processes. The first example was the study of a complex genetic interaction network using synthetic genetic array (SGA) methodology (Tong, Evangelista et al. 2001). Genetic interactions are identified when deletion of two genes results in enhancement or reduction in phenotype that is greater than the expected single additive effect of each deletion. The most extreme type of genetic interaction, synthetic lethality, occurs when combination of deletions of two viable gene deletions results in death. Such studies of genome-wide genetic interactions have identified genes that function in the same pathways, in buffering pathways, in related processes, as well as in prediction of function of novel genes based on the fact that genes which contribute to the same processes usually show genetic interaction to one another (Forsburg 2001).

Because of its importance to this dissertation, synthetic genetic array (SGA) analysis is described here in more detail, as a method utilised to study genetic interactions in a genome-wide manner. In brief, a gene of interest is chosen based on the cell biology one wishes to study. In the deletion strain of this gene, the gene 
is replaced with an antibiotic selection marker cassette, thus generating a "query strain". This query strain is then mated against the non-essential genome-wide deletion mutant array (DMA) in which each gene has been systematically deleted and replaced with a different antibiotic selection marker. The diploid from this mating is sporulated to produce haploid cells, which are subjected to a sophisticated screen that selects for haploids of a specific mating type with the result of haploid strains each containing a query gene deletion and non-essential gene deletion genome-wide. A specific mating type must be selected to prevent opposite mating type haploids mating to form diploid cells. For genetic interaction, the growth phenotype of each deletion is scored and DMA deletions that show synthetic lethality in combination with the query gene deletion are scored as genes which genetically interact with the query gene. SGA has been used to study global genetic interaction in yeast (Tong, Lesage et al. 2004; Costanzo, Baryshnikova et al. 2010) as well as has many other specific biological processes such as protein folding and the secretory pathways (Schuldiner, Collins et al. 2005; Jonikas, Collins et al. 2009; Bircham, Maass et al. 2011), the cellular chaperone network (Zhao, Davey et al. 2005), and the yeast histone modification network (Lin, Qi et al. 2008). Experimentation with haploid cells allows us to reduce the allelic effects of the same genes found in diploid cells which can complicate the interpretation of the genetic -interactions between two genes.

The SGA-based method of creating double mutants in high-throughput can also be modified, and combined with fluorescent reporters to investigate the subcellular morphological effects of different stimuli such as chemicals or mutations. Bircham et al 2011 investigated genes which play roles in the secretory pathway by observing the subcellular localisation of plasma membrane proteins tagged with the green fluorescent protein (GFP) in the different genetic background provided by the DMA (Bircham, Maass et al. 2011). Because resistance mutations to certain chemicals can act in lieu of antibiotic selection markers, and because the yeast genome deletion array is arrayed according to the chromosome position of genes, it is possible to use the DMA as a high-density genetic map. Thus the SGA method can be modified and used as a way to identify both mutations and multigenic traits without the necessity of cloning with plasmids (Jorgensen, Nelson et al. 2002). Jorgensen et al utilised this method to identify SSD1 as the 
suppressor of the lethality associated with defects in the Cbk1 signalling pathway, and named this method "synthetic genetic array mapping" (SGAM).

It was the purpose of the foregoing to show that systematic genome-wide screening is a powerful genetic tool that can be used to investigate the molecular basis of genetic complexity, including drug resistance. It is a major aim of this dissertation to further develop these methodologies that may help identify components of many important biological processes in a way that is faster and less labour intensive than classical genetics and biochemistry.

\section{Section II: Genomics and drug target identification}

Genomic methodologies have proved to be useful for bioactive target identification and mode-of-action characterization. Both allow us to understand the side effects of the compound, as well as help to increase efficacy and potency of future drug design. Target identification is eventually required for drug approval, but can be quite difficult when using biochemical methods. There are a number of genomic methodologies, which have been developed for the identification of drug targets. These include affinity capture, in which the "target is purified from cellular extract using an immobilized or radio-labeled version of the chemical" (Baetz, McHardy et al. 2004; Bradner, McPherson et al. 2006).

Another technique, based on a similar idea to affinity capture, is a protein array in which purified protein molecules are arrayed on a glass slide, and thus can be used to study protein-protein, or ligand-protein interaction (Huang, Zhu et al. 2004).

DNA microarray-based methods are another form of genomic approach. The yeast deletion mutant array (DMA), has unique DNA barcodes flanking each ORF deletion (Winzeler, Shoemaker et al. 1999), thus enabling identification of individual strains within a pooled population (Giaever, Shoemaker et al. 1999; Lum, Armour et al. 2004). Using the DMA, pooled (multiplexed) genome-wide screens of deletion mutants that display drug hypersensitivity allows the identification of pathways that compensate or "buffer" genes affected by drug toxicity in a process known as chemical-genetic interaction profiling (Parsons, Brost et al. 2004; Parsons, Lopez et al. 2006). This multiplexed approach is extremely efficient in 
terms of using scarce test-drug resources. The assumption behind the chemical genetic approach is that small molecules may bind to and inhibit specific proteins, thus acting in lieu of a mutation in that gene to elucidate the biological processes that gene is involved in (Figure 1-1). Chemical-genetic interactions, which may occur between an inhibitory drug and a deletion mutant, can appear as synthetic lethal (SL) interactions, giving us insight into the mode-of-action of the inhibitor, and the protein/gene involved. Similarly, to genetic interaction profiles, where genes that interact with one another tend to have similar functions, small molecule inhibitors may show a synthetic lethal profile with genes that are related to the drug target. A comparison of interaction profiles that have a gene deletion as a "query" with those that have a small molecule inhibitor as a "query" provides revealing information about the drug target. This information is very useful in studying novel compounds with unknown mode-of-action (Parsons, Brost et al. 2004; Parsons, Lopez et al. 2006). Nevertheless, chemical genetic profiling does not necessarily reveal the direct drug target. Information that we can obtain from chemical-genetic profiling is that small molecules that affect the same/similar pathway tend to have similar profiles (Figure 1-2). By comparing the chemicalgenetic profile of known compounds to novel ones, profile similarity information can be used to predict pathways that are affected by novel compounds.

Another recent genomic application of DNA genome-wide deletion mutant arrays is "haploinsufficiency profiling" (HIP). This technique, developed by Giaever et al. (1999), is based on the assumption that reducing the number of gene copies encoding the drug target will result in an increased sensitivity to the drug compared to diploid wild-type cells. Haploinsufficiency profiling has many advantages, such as identifying potential drug targets directly (Lum, Armour et al. 2004). However, HIP also has limitations, since in practice it can only be used to study essential genes, because non-essential genes tend to have back-up pathways. Other limitations include the fact that the "noise" of false positives or false negatives is high (Lum, Armour et al. 2004), and that protein production is not necessarily correlated with the gene dosage effect of haploinsufficiency. 
a
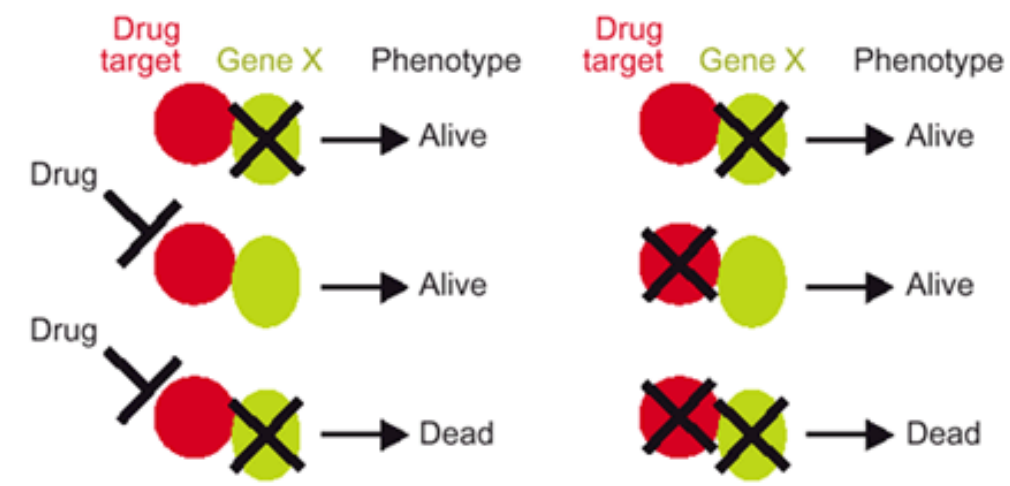

b

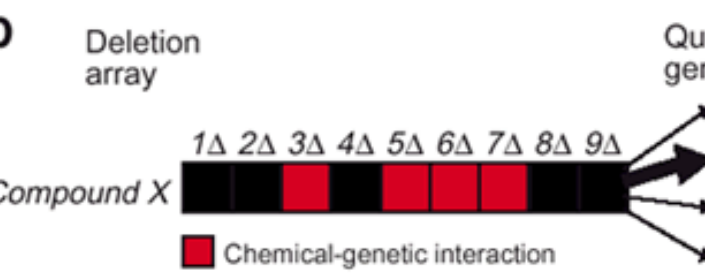

\section{Query Deletion array}

genes $1 \Delta 2 \Delta 3 \Delta 4 \Delta 5 \Delta 6 \Delta 7 \Delta 8 \Delta 9 \Delta$

Chemical-genetic interaction
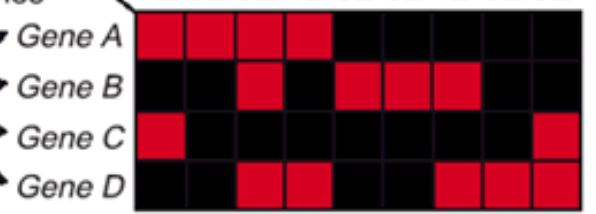

Synthetic lethal interaction

Figure 1-1: Chemical genetic interaction.

(a) Right panel: the drug target interacts with the product of gene $X$, and the two buffer each other effect, so that deletion of either the drug target gene or gene $X$ is non-lethal, but the deletion of both genes leads to lethality. (a) Left panel: a small molecule (drug) inhibits its target and act in lieu to the deletion of the drug target gene. Cells that contain a deletion in gene $X$ in the presence of the drug will show the same phenotype as cells that contain both deletions in the drug target gene and gene $X$-lethality (red block). (b) Comparing the chemical-genetic profile of the compound of interest with the genetic-genetic interaction profiles of genes across the genome can lead us to identify the drug target, or genes in the pathway which is affected by the drug (their profiles should be similar). Figure from Parsons, A. B., R. L. Brost, et al. (2004). 


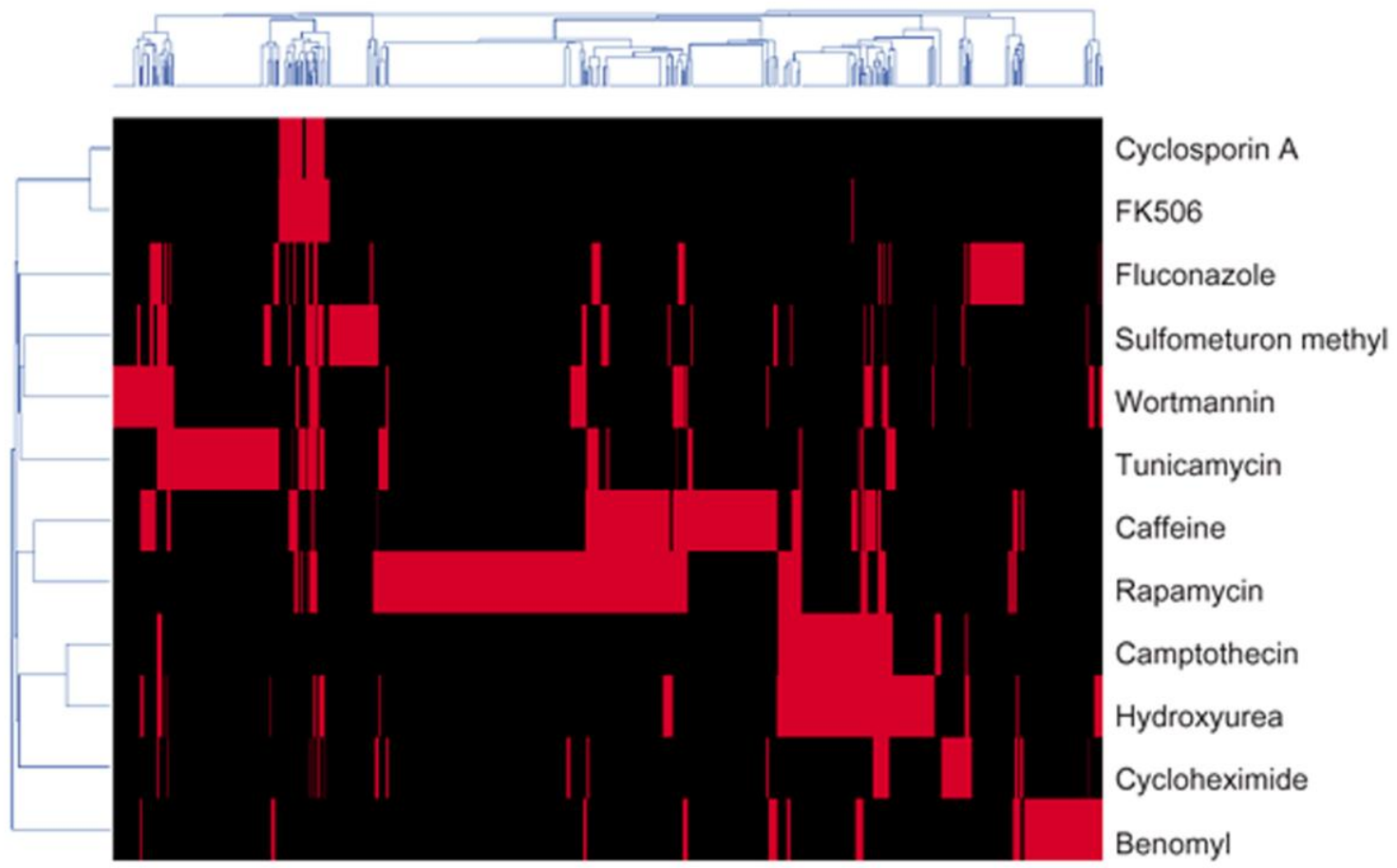

Figure 1-2: Drugs with similar mode-of-action show similar chemical-genetic profiles.

For example, cyclosporin A and FK506 are both immunosuppressive drugs and show similar chemical-genetic profiles (similar sets of genes involved in buffering drugs toxicity). A similar match is observed with camptothecin and hydroxyurea, both of which affect DNA integrity. Camptothecin inhibits DNA enzyme topoisomerase $I$, while hydroxyurea inhibits enzyme ribonucleotide reductase. Diagram modified from Parsons, A. B., R. L. Brost, et al. (2004).

A commonly used method to identify targets is through the generation of drugresistant mutants. Complementation cloning relies on finding a condition where the drug resistant mutant does not grow, e.g. minimal media, temperature sensitivity, various carbon sources, formamide etc. A plasmid-based genomic library can then be used to complement the growth defect, i.e. a gene that can rescue the growth defect should be the one that gives rise to drug-resistance when mutated (Parsons, Lopez et al. 2006). Another way of identifying the drug-resistance gene is to transform wild-type strains with a plasmid-based genomic library derived from a resistant mutant. A mutant gene which can rescue a wild-type growth defect in the presence of drug should be the target of the drug (Miyamoto, Machida et al. 2002). The first method has the disadvantage that we must identify a condition where the resistant mutant will not grow, while the second method is extremely time consuming, labor intensive, and must be repeated for every mutant. In addition, both methods are plasmid-based method, which can be time consuming; 
there are difficulties around the construction of plasmid libraries and getting full coverage of the genome.

An emerging field to identify the mechanism of drug action and resistance is mRNA sequencing (RNAseq). In this technique drug resistant clones are selected, mRNA from the resistant clones and parental clones are then massively parallel sequenced. Bioinfomatics analysis is used to identify the high frequency mutated genes as potentials drug resistant loci (Figue1-21) (Wacker, Houghtaling et al. 2012). This method, although very fast and allows chemical modifications of the drug of interest, relies on powerful bioinformatics to distinguish between natural occurring single nucleotide variations, and the nucleotide changes that cause drug resistant.

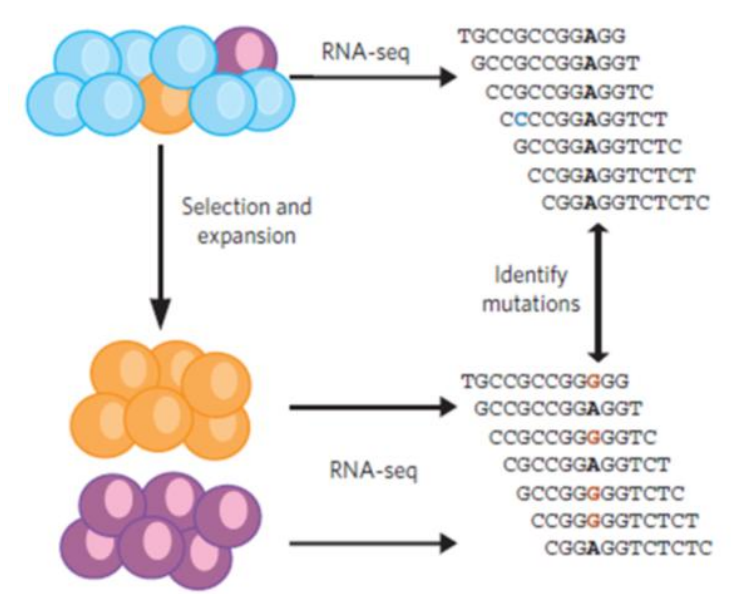

Figure 1-3: Schematic presentation of RNAseq as technique to identify drug resistant loci Image from Wacker, Houghtaling, et al. (2012).

In seeking drug targets of novel compounds, especially natural products, small quantities of the novel material is a major limitation; thus, finding a condition that mimics a drug to determine the resistance locus by complementation is a viable stratagem, but can be a vast wasted effort if a condition is not found. We therefore employed an alternative genome-wide strategy to identify the resistance locus of a drug (neothyonidioside) by using the linkage disequilibrium group that characteristically surrounds the query gene interactions in SGAs. This technique, known as "SGAM" (Jorgensen, Nelson et al. 2002), reasons that if a drug is potently cytotoxic, then resistant mutants are equivalent to antibiotic markers and the drug can be used in lieu of an antibiotic for selection. The drug resistant strain 
is therefore used as a query strain and is crossed into the DMA background by mass mating, creating a comprehensive set of haploid double mutants (Tong, Evangelista et al. 2001). This technique relies on the fact that in haploids, the drug target locus can only be occupied by the mutated drug target that confers drug resistance, or the antibiotic marker from the DMA, but not both. Therefore, growing the DMA mutant that corresponds to the drug target on both the antibiotic and an inhibitory concentration of the drug will result in no growth. Gene deletions closely linked to the query gene form double mutants that, though they are not the target, still show reduced growth, owing to linkage disequilibrium with the target gene, in media containing the combined antibiotic and toxic drug. Because the strains in the DMA are ordered according to chromosome position, this "linkage group" is clearly visible on SGA plates and can be used for mapping of the mutant gene ("SGAM") at a single gene resolution (Jorgensen, Nelson et al. 2002). In this way, SGAM may be utilized to map the locus that confers resistance to neothyonidioside to just a few possible gene candidates i.e. middle of the linkage group in just a few assay steps. We can then sequence relatively few candidate genes for point mutations instead of sequencing the entire genome or transcriptomes (RNAseq).

\section{Section III: Genomics and multidrug resistance}

Organisms require a very particular internal cellular environment in order to function, grow and reproduce. However, cells can encounter various environmental conditions which lead to stress, such as oxidative stress, heat shock, hyper-and hypo-osmotic stress, and toxin challenge (either from cellular metabolism or from external sources). To maintain the optimal internal environment, cells develop various mechanisms to protect from, cope with, or adapt to stress. These protective mechanisms involve many types of proteins and transcription factors, which can be activated in response to stress, and then go on to rapidly activate many more target genes (Gasch, Spellman et al. 2000). Many of these pathways involve signaling, which, on sensing the stress, then relay the information to the nucleus (Hanlon, Rizzo et al. 2011). For example, the osmosensor for sensing osmotic stress, known as MAPK signal transduction, activates a range of general stress-response transcription factors. Other pathways, 
such as the oxidative stress response pathway, make use of enzymes that convert toxic metabolites to non-toxic products (Jamieson 1998; Ikner and Shiozaki 2005), and some of pathways utilise certain membrane protein transporters in cellular detoxification by effluxing toxic compounds/metabolites. An example of one of these pathways is the multidrug resistant stress response (Kuchler and Schüller 2007).

Multidrug resistance (MDR) is usually defined as the ability of a living cell to show resistance to a wide variety of structurally and functionally unrelated compounds. This phenomenon is conserved in bacteria to humans and allows organisms to be able to survive or defend themselves against a variety of cytotoxic compounds in the environment. MDR is also one of the main mechanisms by which pathogenic fungi or cancer cells develop drug resistance.

The MDR response can be mediated in many ways such as mutation in drug targets, inactivation of drugs, reduced cellular uptake of drugs by reduction of membrane permeability (Kuchler and Schüller 2007), Figure 1-4. However, the most commonly observed MDR response is mediated through overexpression of membrane-spanning efflux pumps. Two major classes of transporters that play important roles in MDR are the ABC (ATP binding cassette) family of transporters that are ATP-dependent, and Major Facilitator Superfamily (MFS) transporters. In many organisms the master regulator of MDR pump expression are transcription factors belonging to the Zinc Cluster protein class, which are further detailed below (Gottesman, Fojo et al. 2002; Sipo and Kuchler 2006; Gulshan and Moye-Rowley 2007). 


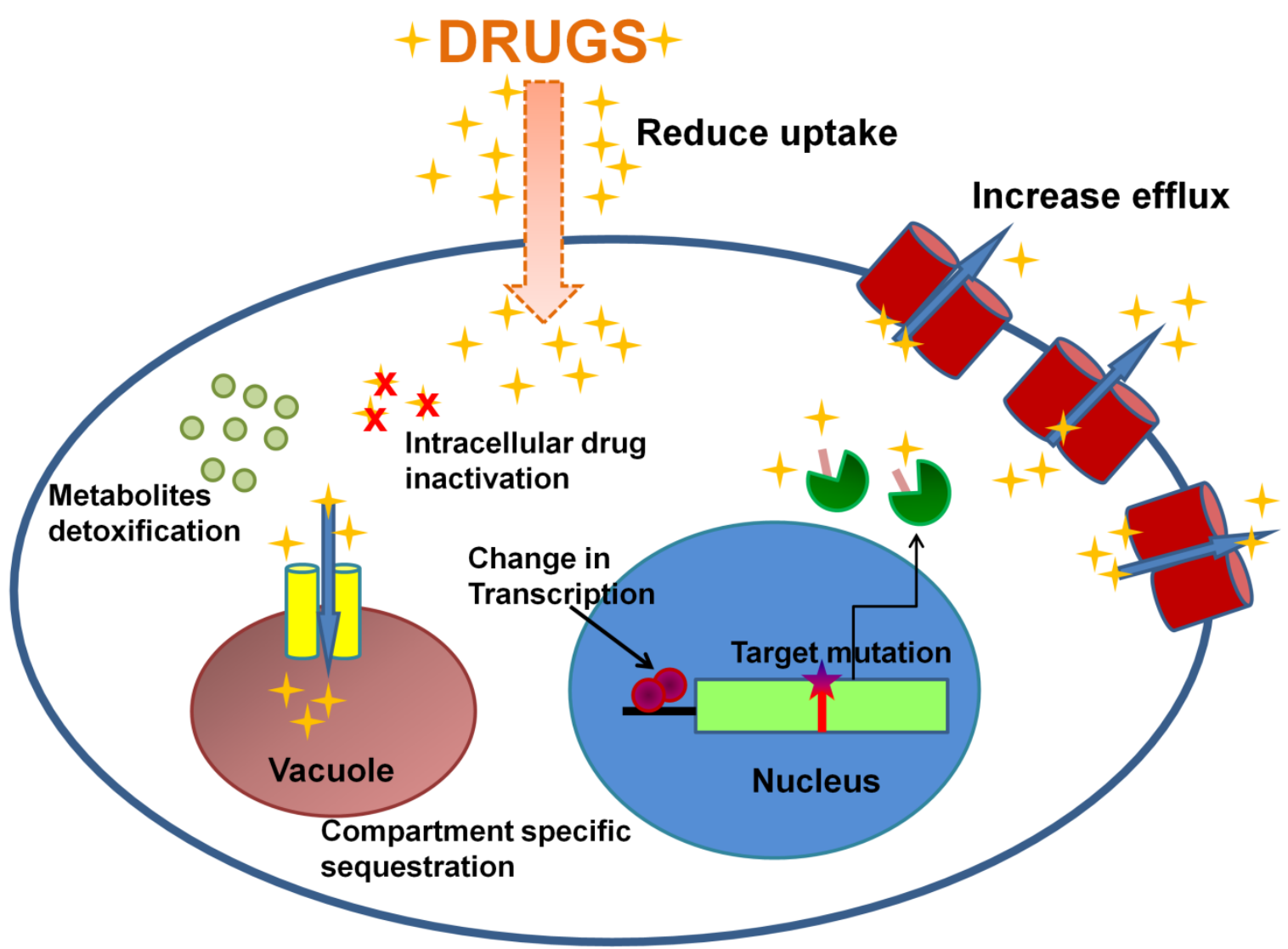

Figure 1-4: Mechanisms of multidrug resistance.

Cells can achieve the MDR phenotype in many ways such as reducing cellular uptake, changing membrane permeability, using of molecular scavengers, intracellular inactivation of drugs, sequestering drugs to specific compartments where they can be destroyed or effluxed out of the cells, changes in transcription or mutation in the target genes, and increased expression of efflux pumps. Diagram modified from (Kuchler and Schüller 2007)

Multidrug resistance has been widely studied in budding yeast and S.cerevisiae shows a great range of small molecule resistance (Gulshan and Moye-Rowley 2007). In yeast, MDR is referred to as pleiotropic drug resistance (PDR).

Although, the transcription factors (Pdr1p/Pdr3p,discussed below) and ABCtransporters (Pdr5p, Snq2p, and Yor1p discussed below) involved in PDR have been widely studied, the way in which cells sense the presence of the drugs, relay this information to the transcription factor and how the transcription factors themselves are controlled are still unclear. As discussed below there are many transcription factors from other stress response pathways that seem to contribute to the PDR phenotypes and might act in either Pdr1p/Pdr3p-dependent or 
Pdr1p/Pdr3p-independent manners; how they actually contribute to the phenotype is also unclear.

In this project, we probed how cells sense the presence of xenobiotic drugs (toxic or inhibitory small molecules in the environment), by investigating possible new regulators/modulators of $\mathrm{Pdr} 1 \mathrm{p} / \mathrm{Pdr} 3 \mathrm{p}$ (Chapter 4). Another concept that we investigated (Chapter 5) was the relationship between PDR and Yap1p, an oxidative stress transcription factor which has been shown to confer resistance to many drugs when overexpressed (Wendler, Bergler et al. 1997). Recognition sequences for Yap1p are also found on main PDR ABC-transporter genes PDR5 and SNQ2, which suggests that it controls many major facilitators of the PDR pathway (Kolaczkowska and Goffeau 1999) (discussed below). We investigated if there are any common regulators between Yap1p and Pdr1p/Pdr3p, which could in turn lead to cross talk between oxidative stress response and the PDR response. The possible crosstalk between these two processes has been suggested in numerous papers and reviews (Miyahara, Hirata et al. 1996; Wendler, Bergler et al. 1997; Zhang, Cui et al. 2001; Moye-Rowley 2003). We then investigated another mechanism of drug resistance that does not require Pdr1p/Pdr3p or activation of ABC-transporters (Chapter5), but involves the sterol composition of the plasma membrane, and how a drug-like compound may exert its cellular toxicity via endocytosis and vacuole formation pathway.

\section{PDR1 and PDR3; the main transcription factors in PDR}

PDR gene expression is rapidly induced in the presence of xenobiotics ( toxic or inhibitory small molecules under the control of two transcription factors, Pdr1p and Pdr3p that are members of the $\mathrm{Zn}_{2} \mathrm{Cys}_{6}$ family of transcription factors (Balzi, Chen et al. 1987; Balzi and Goffeau 1995; DeRisi, van den Hazel et al. 2000; Gulshan and Moye-Rowley 2007). PDR1 was first identified in 1987 by Balzi et al. as a gene which regulates the resistance to various small molecules such as cycloheximide and oligomycin in S. cerevisiae (DeRisi, van den Hazel et al. 2000). In 1986 Subik et al. mapped the locus of PDR3 which encodes another zinc finger transcription factor controlling the PDR response in budding yeast (Šubik, Ulaszewski et al. 1986). Co-immunoprecipitation and chromatin 
immunoprecipitation experiments showed that Pdr1p and Pdr3p can form both homo and heterodimers and Pdr1p constitutively binds to its target promoters in vivo (Mamnun, Pandjaitan et al. 2002; Fardeau, Lelandais et al. 2007). These two zinc cluster proteins are $36 \%$ identical to each other and contain six highly conserved cysteines that bind zinc, a C-terminal acid activation domain and a DNA binding domain (Delaveau, Delahodde et al. 1994). This domain recognizes a DNA sequence known as the pleiotropic drug resistance element (PDRE) that contains the consensus sequence TCCGCGGA (MacPherson, Larochelle et al. 2006). One or several PDREs are found within the 5' UTR of their target genes, mostly ABC pumps, such as PDR5, SNQ2, YOR1, PDR10, and PDR15, each of which is described more fully below and is known to be involved in the PDR response (MacPherson, Larochelle et al. 2006). Other major targets of Pdr1p and Pdr3p are MFS genes such as RTA1, HXT2, HXT9, HXT11, TPO1, RSB1, and some genes involved in lipid homeostasis such as PDR16 and IPT1 (Kolaczkowska and Goffeau 1999). These targets of Pdr1p and Pdr3p overlap but are not identical. For example, Pdr15p which is a close homologue to Pdr5p is more responsive to Pdr3p, and while deletion of $P D R 1$ causes reduction of mRNA level of PDR5, the mRNA level of PDR15 is shown to increase in this background (Wolfger, Mahe' et al. 1997). Pdr1p is also active under normal growth condition because one of its targets, Pdr5p, is a highly expressed protein, suggesting that Pdr1p has a partially constitutive role (Mamnun, Schuller et al. 2004). The difference in transcriptional regulation between Pdr1p and Pdr3p might be due to post-translational modifications. Pdr1p is possibly post-translationally regulated by Ssz1p (Pdr13p), a member of Hsp70 family in yeast (Hallstrom, Katzmann et al. 1998; Eisenman and Craig 2004) while Pdr3p is shown to be controlled by signals from mitochondria as well (Hallstrom and Moye-Rowley 2000; Devaux, Carvajal et al. 2002).

Although a single PDRE is sufficient to respond to Pdr1p/Pdr3p transcriptional activation (YOR1 contains only a single PDRE in its promoter), it has been shown that multiple PDREs are required for full promoter activity, at least in PDR5 (Katzmann, Hallstrom et al. 1996). Interestingly, PDR3 also contains two PDREs in its promoter as does $Y R R 1$, another $\mathrm{Zn}_{2} \mathrm{Cys}_{6}$ zinc-finger transcription factor involved in multidrug resistance response which will be discussed later (Sipo and 
Kuchler 2006). These PDREs act in auto-regulation as well as in response to Pdr1p regulation (Delahodde, Delaveau et al. 1995; Moye-Rowley 2003). Experiments have shown that the mRNA level of PDR3 increased in the hyperactive form of PDR1 (the PDR1-12 mutation confers hyper-resistance to diazaborine) and that the autoregulation process is relevant for cycloheximide response (Delahodde, Delaveau et al. 1995; Wehrschütz-Sigl, Jungwirth et al. 2004). Wehrschütz-Sigl et al. also showed by Northern Blot that the mRNA level of $P D R 1$-12 was increased in this strain compared to wild-type; however they did not detect an increase in expression of a lac $Z$ reporter linked to the $P D R 1$ promoter in the PDR1-12 strain as expected. They suggested that the higher PDR1-mRNA level in this mutant strain might be due to higher mRNA stability and other transcriptional stimulation at the PDR1 promoter.

Figure 1-2 shows the possible way that Pdr1p might regulate the expression of other PDR transcription factors such as PDR3, and YRR1lt is possible PDR1 expression may be subjected to other modifications such as ubiquitination or activation by mediator complexes. Pdr1p then regulates the transcriptional expression of $P D R 3$ and YRR1 as well as other PDR genes (ABC transporters, MFS etc.), all containing PDRE in their promoter regions. Pdr3p is autoregulated as well as regulating YRR1 and other PDR genes that might overlap with Pdr1p. Yrr1p is activated by both Pdr1p and Pdr3p and transcriptionally activates other PDR genes, and therefore, amplify the signal input.

There are about 200 genes which contain PDRE in their promoter regions, but only $10 \%$ of these genes respond to a hyperactive form of PDR1 and/or PDR3 (discussed below) (Fardeau, Lelandais et al. 2007), which is unexpected if Pdr1p/Pdr3p are the sole controllers of the PDR response. This fact suggests that there may be other modulators that are required, in addition to the presence of PDRE, that are necessary for transcriptional activation by Pdr1p/Pdr3p. 


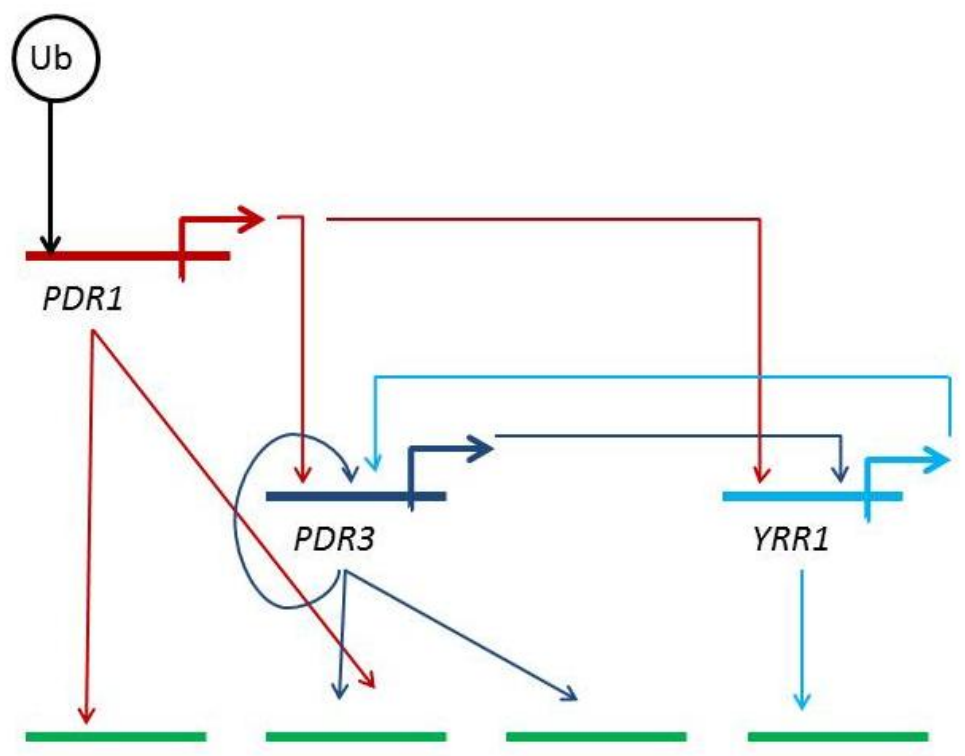

Different PDR genes (ABC transporter, MFS etc.)

Figure 1-5: Possible regulation PDR transcription factors PDR1, PDR3 and YRR1. "Ub" equals sensory input. Adapted from Bolouri \& Davidson, 2002.

Multidrug resistance in fungi is often achieved via change in activity of transcription factors, mainly, Pdr1p and Pdr3p. Loss of either PDR1 or PDR3 results in differential drug tolerance, and loss of both $P D R 1$ and $P D R 3$ results in severe drug hypersensitivity(Kolaczkowska and Goffeau 1999). Gain-of-function pdr1 and pdr3 mutant alleles produce hyperactive Pdr1p and Pdr3p, which in turn overproduce their target efflux pumps, and therefore confer a multidrug resistance phenotype (Kolaczkowska and Goffeau 1999; Gulshan and Moye-Rowley 2007). Many single point mutations in both $P D R 1$ and $P D R 3$ have been identified that cause hyperactive transcriptional activity, such as pdr1-2, pdr1-3, pdr1-6, pdr1-7, pdr1-8, pdr3-2 to pdr3-10. Of these, pdr1-3 mediates the highest increase in resistance to many compounds, such as cycloheximide, oligomycin, 4-nitroquinoline- $\mathrm{N}$-oxide and ketoconazole ((Carvajal, van den Hazel et al. 1997). Polymorphic forms of PDR1, pdr1-11, and pdr3-11 also disrupt the inner bilayer leaflet steady-state and cause translocation of phosphatidylethanolamine, across the plasma membrane, leading to the notion that control of phospholipid composition is also regulated in multidrug resistance. Indeed, it has been shown by many experiments that normal multidrug resistance in fungi depends on the lipid homeostasis (Kolaczkowska and Goffeau 1999; Schüller, Mamnun et al. 2007; Shahi and Moye-Rowley 2009). This will be discussed in later chapters. 


\section{Membrane transporters in pleiotropic drug resistance}

In yeast, the main targets of Pdr1p and Pdr3p that cause the majority of PDR phenotypes are ATP-binding cassette $(A B C)$ drug transporters (Sipo and Kuchler 2006). This family of proteins is conserved from bacteria to humans. $A B C$ transporters are present in all cellular compartments except the endoplasmic reticulum (ER) and nuclear membrane (Figure 1-6) (Wolfger, Mamnun et al. 2001)

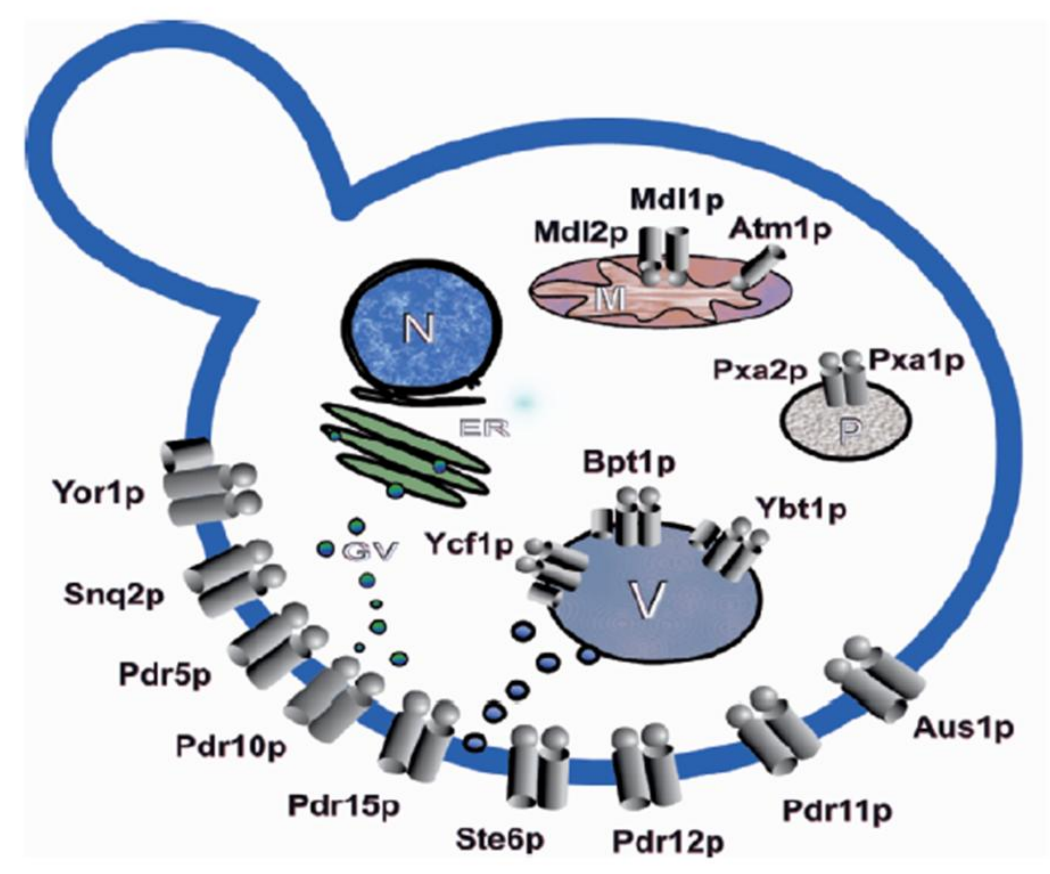

Figure 1-6: Membrane ABC transporters in yeast and their locations.

Figure from (Kuchler and Schüller 2007) with permission.

All $A B C$ proteins share similar hallmark domain organization, shown in Figure 1-7 which includes at least one conserved ATP-binding cassette (ABC), also known as nucleotide binding domain (NBD), and several $\alpha$-helical membrane-spanning segments (transmembrane segment, TMS) (Wolfger, Mamnun et al. 2001). $S$. cerevisiae ABC-proteins are often used as a model to study the clinical problem of drug resistance in infectious disease and cancer as well as in pharmaceutical screens for novel drugs (Gottesman, Fojo et al. 2002; Golin, Ambudkar et al. 2003; Simon and Bedalov 2004; Jungwirth and Kuchler 2006; Kuchler and Schüller 2007; Xia, Yang et al. 2010). The most prominent and well-characterized ABC transporters in yeast that are targets of Pdr1p/Pdr3p are Pdr5p, Snq2p, and Yor1p. These plasma membrane proteins have broad specificity to structurally and 
functionally unrelated compounds, which they "pump" out of the cell in an ATPdependent manner.

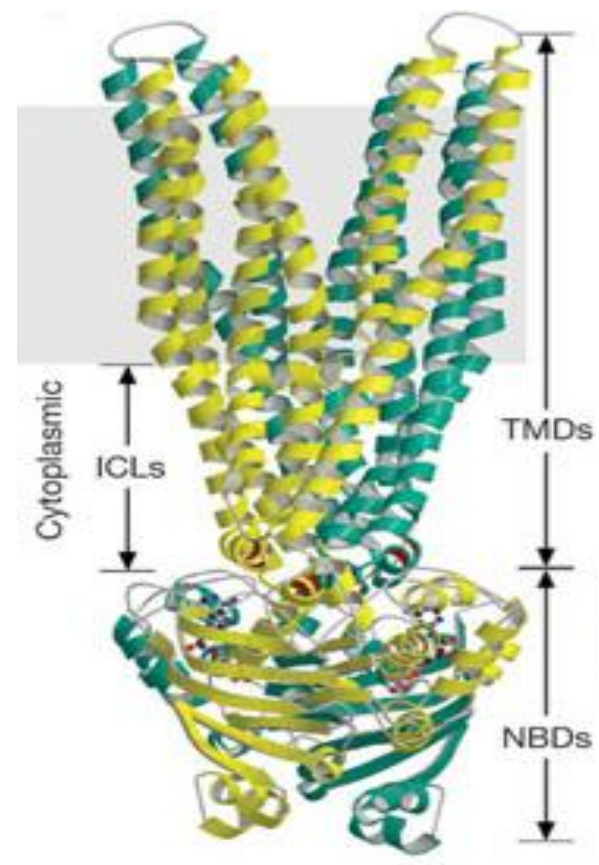

Figure 1-7: multidrug $A B C$ transporter structure of Staphylococcus aureus (Sav1866).

TMD: transmembrane domain. NBD: Nucleotide binding domain. ICL: intracellular loops (between transmembrane helices). Image from (Dawson RJ and Locher KP 2006) http://www.rcsb.org/pdb.

\section{PDR5}

Pdr5p has a six membrane-spanning domains and a repeated putative ATPbinding domain. It is the functional homologue of mammalian ATP transporters, Pglycoproteins (P-gps), which are upregulated in many cancer cell lines (Balzi and Goffeau 1995; Wolfger, Mamnun et al. 2001; Gottesman, Fojo et al. 2002). The structure of Pdr5p is shown in Figure 1-8 (Rutledge, Esser et al.2001). Pdr5p was purified and characterized from the overexpression of pdr1 mutants (Decottignies, Kolaczkowski et al. 1994). Pdr5p function involves PDR phenotype, as well as cation resistance (Miyahara, Hirata et al. 1996) and lipid translocation (Kihara and Igarashi 2004). 


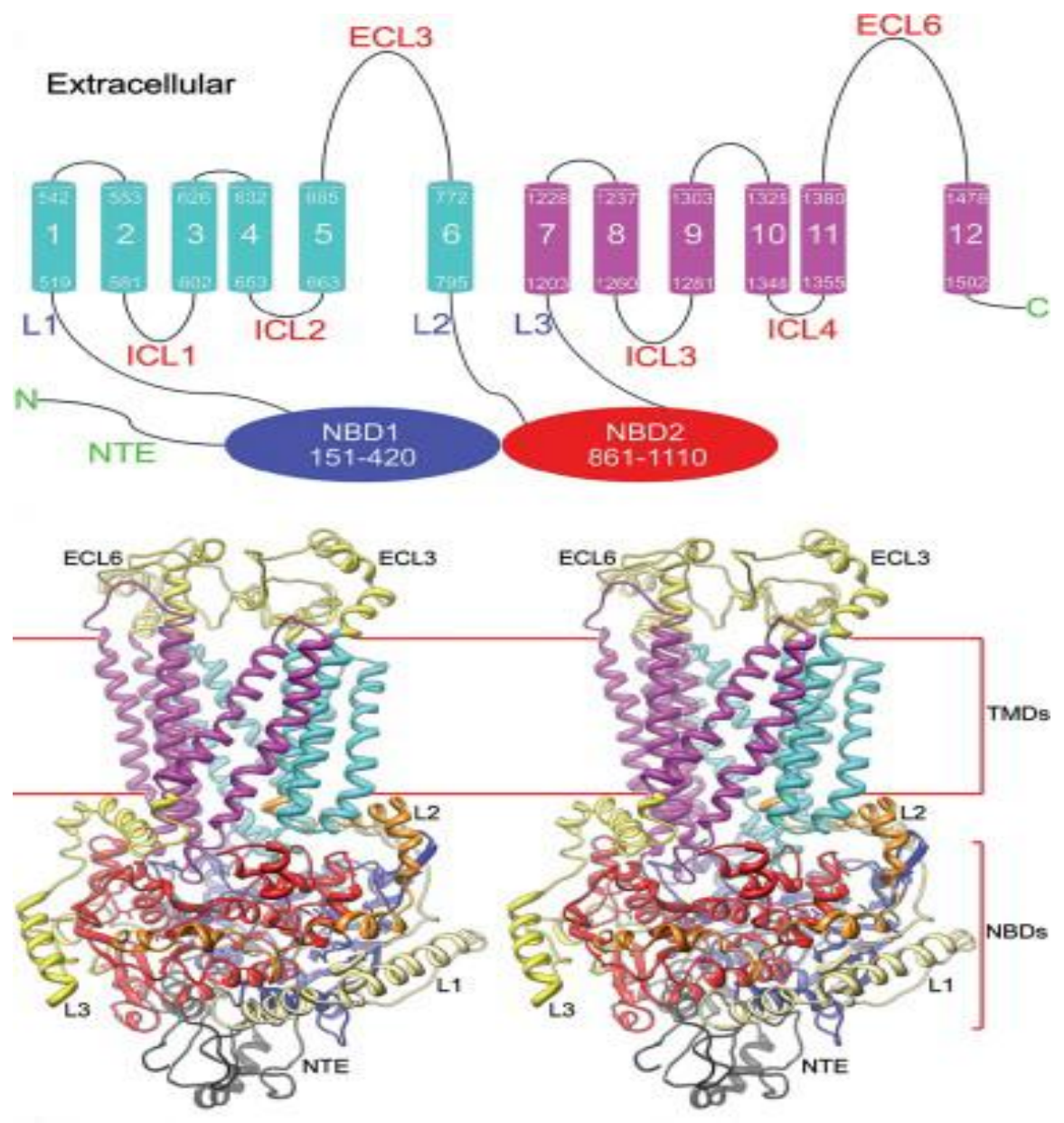

Figure 1-8: Pdr5p structure.

Top: Predicted topology of Pdr5p. Bottom: Stereoscopic view of the Pdr5p model showing two trans-membrane domains: TMDs. Underneath the TMDs are the nucleotide binding domains, NBDs (in blue and red). The NBDs are connected by loops L1-L3 in yellow and orange. Images from (Rutledge, Esser et al. 2011)

Disruption of PDR5 is non-lethal, but causes sensitivity towards many compounds, such as cycloheximide, chloramphenicol, ketoconazole, and sulfometuron methyl. A strain lacking PDR5 also exhibits a metal salt hypersentive phenotype (Balzi and Goffeau 1995; Miyahara, Hirata et al. 1996). Pdr5p is observed in the highest abundance during exponential growth phase and is thought to be involved in detoxification of toxic metabolites, in a similar manner to its closest homologue, Pdr15p. However, unlike Pdr15p, Pdr5p abundance is strongly repressed in stationary phase and Pdr15p seemed to be more dependent on Pdr3p and less dependent on Pdr1p than Pdr5p is (Mamnun, Schuller et al. 2004). Another close homologue of Pdr5p is Pdr10p, sharing 66\% identical residues (Wolfger, Mamnun et al. 2001). Pdr10p was shown to play a role in maintaining the distribution and function of a subset of other plasma membrane proteins (Rockwell, Wolfger et al. 
2009). Cells lacking PDR10 show a defect in maintaining plasma membrane asymmetry, a defect in endocytosis of phosphatidylethanolamine (PE), and chitin synthase Chs3p function (Rockwell, Wolfger et al. 2009).

PDR5 contains at least three known PDREs in its promoter and its expression is positively regulated by Pdr1p and Pdr3p (Balzi and Goffeau 1995; Katzmann, Hallstrom et al. 1996), and negatively regulated by Rdr1p (Hellauer, Akache et al. 2002). Experiments have shown that $P D R 5$ can also be heat-shock induced via the transcription factors Yap1p and Cad1p (Miyahara, Hirata et al. 1996).

\section{SNQ2}

As with PDR5, SNQ2 encodes an ABC transporter that may confer PDR in yeast when overexpressed. It is a functional homologue to the mammalian Pglycoprotein. The Snq2p transporter confers multidrug resistance particularly to mutagens such as methyl-nitro-nitrosoguanidine (MNNG) and 4-nitroquinoline-Noxide (4-NQO) (Servos, Haase et al. 1993; Bauer, Wolfger et al. 1999). Like PDR5, the null mutant of SNQ2 is non-lethal but causes hypersensitivity toward many drugs and metal salts (Balzi and Goffeau 1995; Miyahara, Hirata et al. 1996). However; the null mutation of SNQ2 does not increase in sensitivity toward cycloheximide, a well-known xenobiotic that is removed from cells by Pdr5p, or oligomycin (Decottignies, Kolaczkowski et al. 1994; Gulshan and Moye-Rowley 2007). It has been shown that besides functioning in the PDR response Snq2p also is involved in cation resistance and can be upregulated by the transcription factors Yap1p and Cad1p via binding site in its promoter(Miyahara, Hirata et al. 1996).

The closest homologue of Snq2p is Pdr12p which shares $60 \%$ primary sequence identity (Bauer, Wolfger et al. 1999). However, they have differential expression since $P D R 12$ is highly induced in response to weak organic acids such as sorbate and benzoate rather than to hydrophobic drugs like SNQ2 (Piper, Mahe et al. 1998; Wolfger, Mamnun et al. 2001). Some other stressors such as high osmolarity or ethanol level can also induce PDR12 but the increase in expression is not as pronounced as with sorbate. PDR12 expression induction by cellular stress is also independent from Pdr1p/Pdr3p and does not require other well-known stressresponse transcription factors such as Yap1p or Msn2p. The fact that SNQ2 and 
PDR12 require different inducers despite high similarity in primary sequence suggest that there might be other novel transcription factors control yet to be found.

\section{YOR1}

YOR1 encodes a multidrug-resistance -associated protein (MRP) in a subfamily of plasma membrane ATP-binding cassette $(\mathrm{ABC})$ proteins. The YOR1 null mutation is viable but confers sensitivity to many drugs and metals. It shows significant homology to mammalian MRP and to some extent to the cystic fibrosis conductance transmembrane regulator (CFTR) (Wolfger, Mamnun et al. 2001). YOR1 was identified in the genetic screens for oligomycin and reveromycin $\mathrm{A}$ resistance (Katzmann, Hallstrom et al. 1996). Besides multidrug resistance, Yor1p also plays a role in metal detoxification, for example, that of cadmium, mercury, and arsenite (Katzmann, Hallstrom et al. 1995; Cui, Hirata et al. 1996; Decottignies, Grant et al. 1998; Liu 2011), and also in transportation of toxic compounds such as unconjugated bilirubin, selenodigluthione, and oxidized glutathione (Lazard, Ha-Duong et al. 2011).

Yor1p shares many substrates with Pdr5p and Snq2p but also has distinct substrates preferences (Rogers, Decottignies et al. 2001). Like PDR5 and SNQ2, YOR1 expression is positively regulated by $\mathrm{Pdr} 1 \mathrm{p} / \mathrm{Pdr} 3 p$ through the presence of PDREs in its promoter. While expression of PDR5 is severely affected in the $\Delta p d r 1 / \Delta p d r 3$ background, the expression of $S N Q 2$ and YOR1 is decreased but can still maintain a Pdr1p/Pdr3p-independent expression (Kolaczkowska and Goffeau 1999). PDR5 and SNQ2 both contain binding sites for an AP-1-like basic leucine zipper (bZIP) transcriptional activator such as Yap1p (Yap1p response element, YREs) which are involved in the oxidative stress response, and Cad1p which is involved in stress responses and iron metabolism(Kolaczkowska and Goffeau 1999). These binding sites are not found in the YOR1 promoter region. Yor1p however, can confer oligomycin resistance via activation by Yrr1p, another $\mathrm{Zn}(\mathrm{II})_{2} \mathrm{Cy} 6$ transcription factor involved in PDR which binds to the Yrr1p response element ,similar to PDRE, in the promoter region. Interestingly the Yrr1p binding site is absent in the promoter regions of PDR5 and SNQ2 (Kolaczkowska and Goffeau 1999; Gulshan and Moye-Rowley 2007). 
The closest homologues of Yor1p are Ycf1p and Mrp1p, where both act as glutathione conjugate transporters. Ycf1p plays a role in cadmium resistance and, to some extent, lies under the control of Yap1p (Bauer, Wolfger et al. 1999). Ycf1p also has function in unconjugated bilirubin, selenodigluthatione, and oxidized glutathione transport (Lazard, Ha-Duong et al. 2011).

Interestingly, deletion of the SNQ2 and/or YOR1 open reading frame (ORF) increases resistance to ketoconazole and other compounds that are specific to Pdr5p (Kolaczkowska, Kolaczkowski et al. 2008). Similar results were observed when PDR5 was deleted namely resistance to Yor $1 p$, and Snq2p specific substrates was increased. These data suggest some sort of "compensatory activation" (Kolaczkowska, Kolaczkowski et al. 2008). Such activation of paralogous transporters is Pdr1p-dependent and occurs when one or more of the other transporters are deleted even in the absence of drugs. Compensatory activation is Pdr3p-independent; indeed, deletion of PDR3 stimulates even more induction of this "compensatory activation" suggesting that Pdr3p is a suppressor of this compensatory process. Such observations in general support the idea that even though Pdr1p and Pdr3p share very similar sequences they regulate their (overlapping) downstream targets differently. Moreover, they might be subjected to different regulation and other unknown proteins/ modulators might involve in the upstream regulation of the PDR master regulator Pdr1p/Pdr3p. Figure 1-9 from Davidson EH 2010, shows how a gene regulatory network (GRN) provides "system level explanations of developmental and physiological functions" such as differentiation in different stage of development. Perhaps PDR networks in yeast might employ similar "logic" systems to control the PDR response dependant on combinations of "overall inputs" modifying the overall "remain-on" circuitry shown in figure 1-6 caused by general xenobiotics (initial inputs) where particular kinases are "signal inputs". 


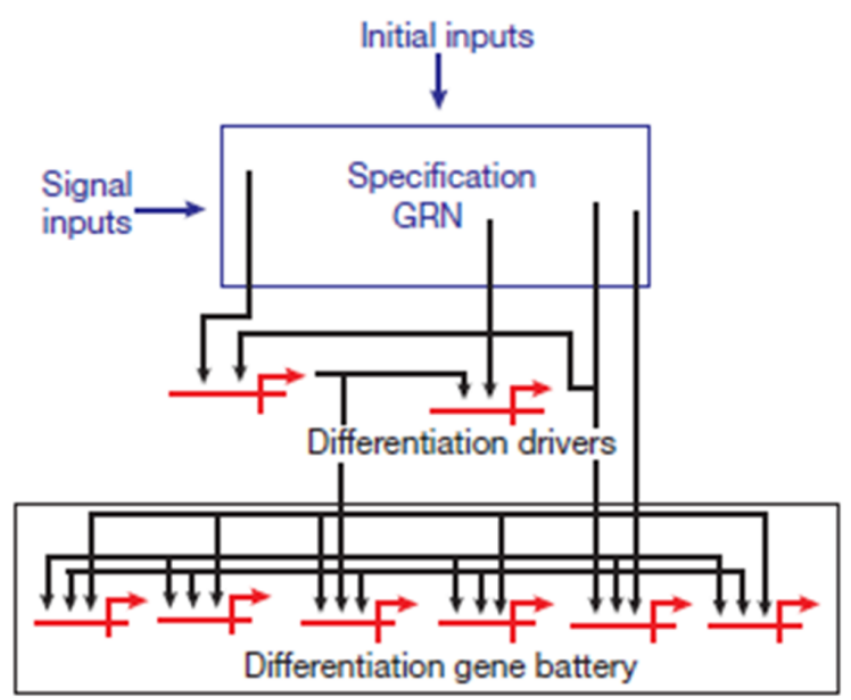

Figure 1-9: Gene regulatory network (GRN)

The Gene Regulatory Network is shown with a master controller and downstream effectors. In multicellular organisms, a specific GRN receives the signal input that can then turn on a few master regulators. Specific downstream effectors encode for cell-type-specific proteins that respond to number of regulatory factors that then produce the final phenotype. There may be cross talk between regulators to activate downstream effectors. It is possible that different ABC transporters (downstream effectors), might be regulated by Pdr1p/Pdr3p and other regulators which are all controlled by unknown upstream GRN. Image from (Davidson 2010).

\section{Major facilitators}

Besides the $A B C$ transporter, another type of transporter that contributes to the PDR phenotype is the major facilitator superfamily (MFS) which is protonmotive-force -dependent. There are at least 20 different MFS proteins that have been shown to be involved in multidrug resistance(Gulshan and Moye-Rowley 2007) and often share overlapping substrate specificity with ABC transporters. An example is Flr1p, a plasma membrane multidrug transporter that contributes to fluconazole and cycloheximide resistance in a similar way to Pdr5p but also pumps out benomyl (Alarco, Balan et al. 1997; Brôco, Tenreiro et al. 1999).

MFS-MDR transporters are found in bacteria, Achaea and eukaryotes. They are classified by the number of predicted transmembrane spans into two families. First is the 12-spanner drug: $\mathrm{H}^{+}$-antiporter-1 (DHA1), and second is the 14 spanner drug: $\mathrm{H}^{+}$-antiporter-2 (Gbelska, Krijger et al. 2006). 
In S.cerevisiae, 12 genes comprise the DHA1 family. These are AQR1, QDR1, QDR2, QDR3, FLR1, DTR1, TPO1, TPO2, TPO3, TPO4, HOL1, and YHK8 (Gbelska, Krijger et al. 2006). Aqr1p, Qdr1-3p and Flr1p are all plasma membrane proteins that are involved in PDR phenotypes with the first four proteins involved in resistance to quinidine, an anti-arrhythmic agent (Nunes, Tenreiro et al. 2001; Tenreiro, Vargas et al. 2005). Tpo1p and Tpo1-4p are plasma membrane proteins and confer resistance to polyamine toxicity by detoxifying excess spermidine and putrescine, produced during the breakdown of amino acids (Sá-Correia and Tenreiro 2002; Albertsen, Bellahn et al. 2003). Tpo1p and Tpo4p can mediate growth resistance to cycloheximide, a well-known Pdr5p substrate, and quinidine similar to Aqr1p and Odr1-3p (Sá-Correia and Tenreiro 2002). Furthermore the null mutant of TPO1 (Atpo1) displays increased sensitivity to nystatin, a wellknown antifungal drug (Sá-Correia and Tenreiro 2002).

There are 10 DHA2 drug: $\mathrm{H}^{+}$-antiporters that have 14 predicted membranespanning segments in $S$. cerevisiae. This group consists of VBA1, VBA2, VBA3, VBA4, VBA5, ATR1, AZR1, SGE1, YML1, and YMR279C.

Vba1-3p are vacuolar membrane proteins involved in basic amino acid transportation (Shimazu, Sekito et al. 2005). ATR1 (former name SNQ1) was one of the first characterized yeast efflux pumps and is required for resistance to aminotriazole and 4-nitroquinoline-N-oxide (Kanazawa, Driscoll et al. 1988; Gömpel-Klein and Brendel 1990). Azr1p, a plasma membrane transporter confers resistance to azole antifungal drugs such as ketoconazole and fluconazole which are also pumped out by $A B C$ transporter Pdr5p. Azr1p also pumps acetic acid ,propanoic acids (weak organic acids), and crystal violet dye (Tenreiro, Rosa et al. 2000; Bauer, Rossington et al. 2003).

Although most DHA1 and DHA2 transporters are involved in the PDR phenotype and have similar primary sequences, only TPO1 contains a PDRE in its promoter and is regulated by Pdr1p. ATR1 and FLR1 are both regulated by Yap1p,the oxidative stress transcription factor which as mentioned above also has a binding site in front of $A B C$ transporters Pdr5p and Snq2p (Kolaczkowska and Goffeau 1999). In fact the FLR1 mRNA level is hardly detectable during yeast exponential 
growth phase, and is highly induced in the presence of benomyl; such induction is highly Yap1p-dependent (Sá-Correia and Tenreiro 2002).

Other proteins in the major facilitator superfamily (MFS) transporters that might be involved in multidrug resistance are the hexose transporters (Nourani, Wesolowski-Louvel et al. 1997; Ozcan and Johnston 1999). MFS proteins can transport glucose down a concentration gradient (Ozcan and Johnston 1999). HXT9, and $H X T 11$ encode nearly identical proteins that are not induced by glucose as other HXT genes (HXT1-4) are, but are under the transcription control of both Pdr1p and Pdr3p where Pdr3p is shown to bind directly to the promoter region of $H X T 11$, probably at a PDRE (Ozcan and Johnston 1999). Interestingly, deletion of one or both of HXT9 or HXT11 increases sensitivity to cycloheximide, sulfometuron methyl, and 4-NQO (Ozcan and Johnston 1999) which is reverse of what normally is observed when Pdr1p/Pdr3p targets, such as PDR5, SNQ2, YOR1, or TPO1, are deleted. Thus, it was speculated that hexose transporters could negatively regulate $A B C$ transporters or are involved in drug uptake itself (Nourani, Wesolowski-Louvel et al. 1997).

\section{Other transcription factor regulators for PDR}

Even though Pdr1p and Pdr3p are the main transcription factors for multidrug resistance in yeast, there are other transcription factors as well as other modulators that play important roles in the PDR phenotype.

For example, Thakur et al. 2008 showed that Pdr1p and Pdr3p directly bind to ketoconazole in the carboxy $(\mathrm{C})$-terminal of DNA binding domain (Thakur, Arthanari et al. 2008), and that a Gal11p/Med15p, a subunit of the RNA polymerase II mediator complex, is required for the xenobiotic response and multidrug resistance. Furthermore, not all ABC transporters involved in PDR are entirely dependent on Pdr1p / Pdr3p. This observation is supported by experiments showing that deletion of both $P D R 1$ and PDR3 greatly reduces the basal level of their main target, Pdr5p but do not eliminate PDR5 induction upon cycloheximide or diazaborine treatment. This is similar to SNQ2 expression in 4-nitroquinoline 1-oxide (4-NQO) which can be Pdr1p/Pdr3p independent (Cui, Shiraki et al. 1998; Wehrschütz-Sigl, Jungwirth et al. 2004). 
Both $A B C$ and MFS genes together with many other genes involved in the control of lipid biosynthesis, contain sites for multiple transcription factors for various stress responses leading to cross pathway regulation events. Gain-of-function of mutations in many transcription factor besides PDR1/PDR3 cause change in plasma membrane composition via their target genes (Lucau-Danila, Delaveau et al. 2003).

To date, there is little known about what may regulate the regulators (Pdr1p/Pdr3p). This dissertation questions whether other transcription factors and proteins play roles in multidrug resistance in $S$. cerevisiae. Figure 1-10 shows possible PDR phenotype regulation in $S$. cerevisiae, and some of the proteins involved in PDR regulation are discussed below.

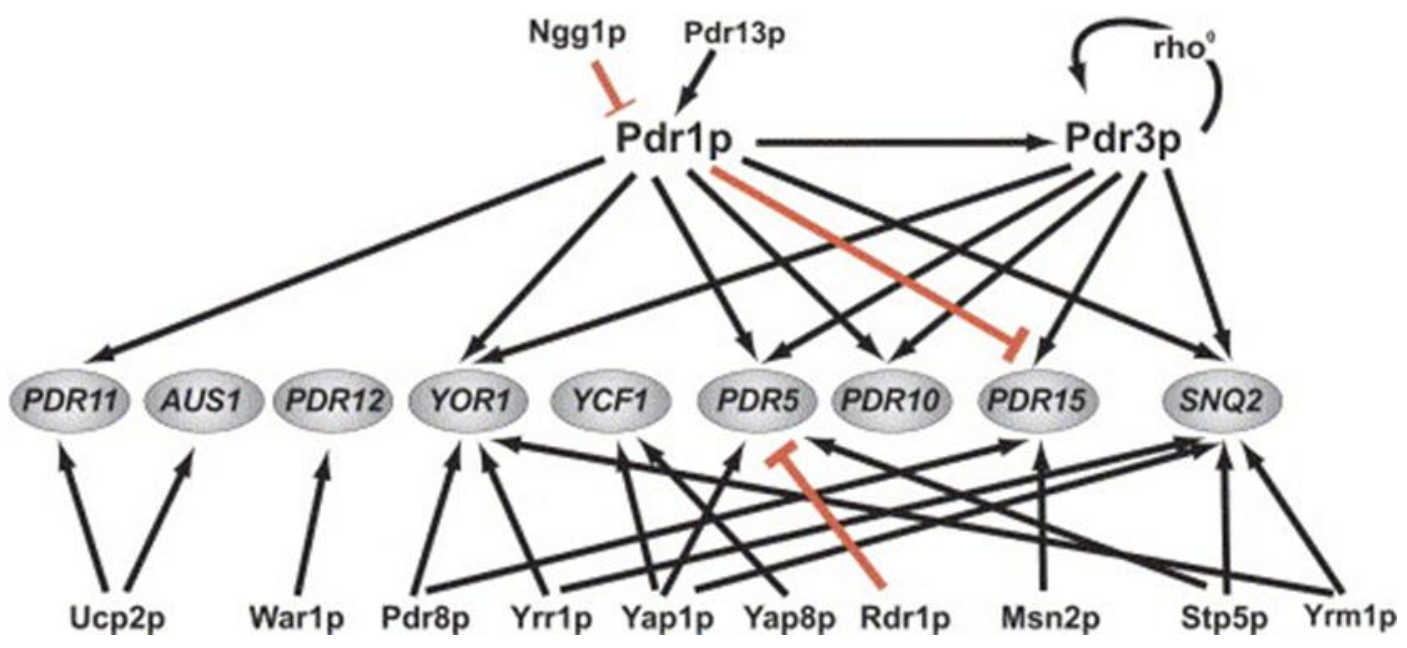

Figure 1-10: PDR regulation in S. cerevisiae.

Besides Pdr1p and Pdr3p, many of the ABC transporters and MFS may be controlled by other transcription factors from other stress responses, such as the Yap1p from oxidative stress response and the Msn2p from general stress reponse. Pdr1p and Pdr3p can also be controlled by other proteins such as Pdr13p (Ssz1p) and SAGA complexes, Ngg1p, as well as mitochondrial status (discussed in the section "Other regulators of PDR", page 28) . Figure from (Jungwirth and Kuchler 2006).

\section{Zinc cluster transcription factors}

\section{YRR1 and YRM1}

Yrr1p is another $\mathrm{Zn}_{2} \mathrm{Cys}_{6}$ zinc-finger transcription factor identified through a mutation that increases resistance to the cell-cycle inhibitor revoromycin A, 4-NQO, and oligomycin (Cui, Shiraki et al. 1998; Zhang, Cui et al. 2001; Moye-Rowley 
2003). Yrr1p mediates drug resistance via binding to the promoter region of the $A B C$ transporter genes, SNQ2 and YOR1, and upregulating them, since loss of YRR1 reduced expression of these two important efflux pumps. There has been defined a Yrr1p response element (YRRE) that is found in front of the coding region of these genes with the consensus sequence of T/ACCGC/TG/TG/TA/TA/T (Le Crom, Devaux et al. 2002), similar to PDRE (5'TCCGCGGA-3'). Other genes with promoters that interact with Yrr1p are SNG1, which encodes a plasma membrane protein, $A Z R 1$, and $F L R 1$. The latter two encode MFS transporters, and FLR1 is also controlled by the Yap1p transcription factor. The promoters of YLLO56C and YLR346C also contain the YRRE. Both of these genes encode putative proteins of unknown function, and the latter is found in mitochondria and also lies under the control of Pdr1p, implying crosstalk between Pdr1p and Yrr1p (Le Crom, Devaux et al. 2002). Similarly to PDR3, YRR1 also has a PDRE in its promoter region and YRR1 expression is autoregulated, as well as being regulated by Pdr1p, and possibly Pdr3p (Zhang, Cui et al. 2001). They showed that although Yrr1p contributes to oligomycin resistance via Yor1p, it does so in a Pdr1p/Pdr3p-dependent manner. Nevertheless, the YRR1 gain of function mutant (YRR1-1) which exhibits drug resistance and increased target expression was found to contain short duplication of residues in the C-terminus that can activate YOR1 gene expression in Pdr1p/Pdr3p-independent manner (Zhang, Cui et al. 2001). The authors speculate that it is possible that the signal from Pdr1p and or Pdr3p was amplified by another transcription factor, like Yrr1p, which shares overlapping target genes with Pdr1p and Pdr3p. Figure 1-10 shows the PDR target genes that are controlled by Pdr1p, Pdr3p, Yrr1p and Yrm1p. Some of the targets are controlled by more than one transcription factor.

The closest YRR1 closest homologue is YRM1 (Lucau-Danila, Delaveau et al. 2003), another $\mathrm{Zn}_{2} \mathrm{Cys}_{6}$ zinc-finger transcription factor. Lucau-Danila et al. have shown using genome-wide expression analysis and chromatin immunoprecipitation that Yrm1p upregulated 23 genes, 14 of which are also regulated by Yrr1p. These include $A B C$ and MFS transporter genes such as AZR1, YOR1, SNG1, and SNQ2. FLR1 is the only Yrr1p target in which its promoter does not interact with Yrm1p. Furthermore, Yrm1p only interacts with 
targets in the absence of Yrr1p, and that gene expression in the 14 common targets as well as gain-of-function mutant of $Y R R 1$ activity are repressed when YRM1 is overexpressed. To make the multidrug resistance phenotype yet even more complex, deletion of YRM1 does not affect expression of YRR1. However, a Yrm1p mutant that has lost its central regulatory region will not recognize the presence of Yrr1p. This suggests that post-transcriptional processes might also play role in transcription factor regulation. Furthermore, Lucau-Danila et al. also suggested that the central middle homology region (MHR) region of $\mathrm{C} 6$ zinc cluster transcription factors might play roles in DNA target discrimination (Schjerling and Holmberg 1996). As mentioned previously, most targets of these two transcription factors are plasma membrane ABC and MFS transporters, as well as genes involved in lipid metabolism that can affect the plasma membrane composition and change sensitivity to different drugs.

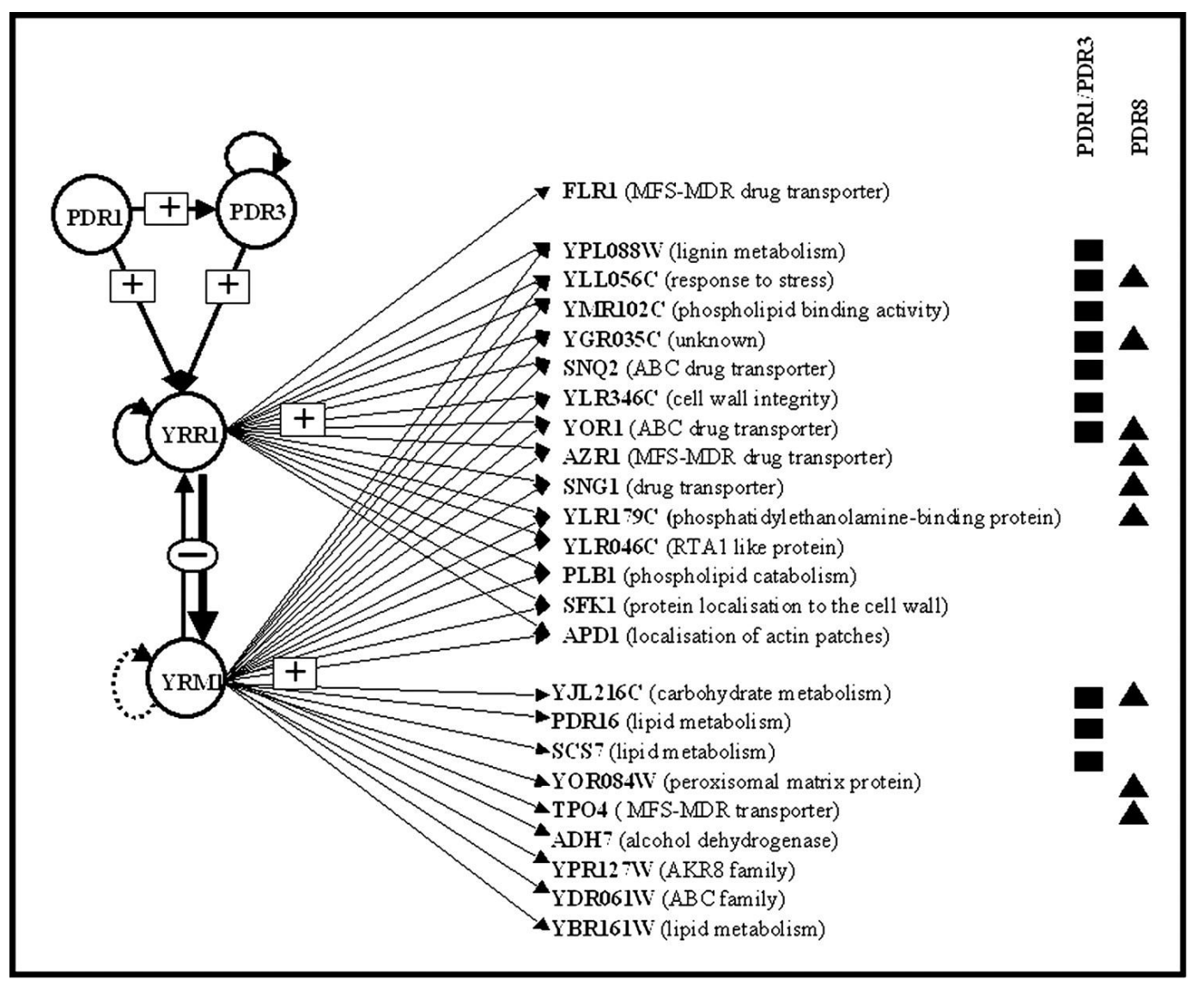

Figure 1-11: Overlapping targets between different PDR transcription factors.

Target genes involved in the PDR phenotype mostly encode plasma membrane proteins. Genes that are controlled by Pdr1p/Pdr3p and Yrr1p/Yrm1p are shown as black squares. Genes that are regulated by Pdr8p and Yrr1p/Yrm1p are indicated by black triangles. Figure from (Lucau-Danila, Delaveau et al. 2003). 


\section{STB5 and RDS1, 2, 3}

Stb5p was found to play roles in multidrug resistance by forming a heterodimer with Pdr1p and binding to PDRE in front of target genes such as PDR5 and SNQ2 (Akache, MacPherson et al. 2004). STB5 encodes for a $\mathrm{Zn}_{2} \mathrm{Cys}_{6}$ zinc finger transcription factor. As well as multidrug resistance regulation, STB5 regulates oxidative stress response via genes in the pentose phosphate pathway, and genes involved in the production of NADPH, which is a metabolite required for oxidative stress resistance (Larochelle, Drouin et al. 2006). Deletion of STB5 causes hypersensitivity toward cycloheximide, even in the presence of Pdr1p and Pdr3p, as well as reduced PDR5 and SNQ2 expression (Akache, MacPherson et al. 2004).

Similarly to STB5, RDS1, 2, 3 are zinc cluster proteins, and strains lacking RDS1 and/or RDS3 are sensitive to cycloheximide, while deletion of RDS2. which involves in regulating gluconeogenesis and the glyoxylate cycle, confers sensitivity toward ketoconazole. PDR5 expression was decreased upon deletion of the RDS genes, and deletion of RDS2 caused a decrease in the PDR16 RNA level (Akache, MacPherson et al. 2004). As discussed above, these transcription factors generate another level of multidrug resistance regulation and interplay between different regulators. However, much is still unknown about the function of these genes.

\section{RDR1}

Rdr1p is a member of the Gal4p family of zinc cluster proteins, which are proposed to act as transcriptional repressors. Deletion of RDR1 results in an inability for cells to grow in non-fermentable carbon sources and causes upregulation of many membrane protein-encoding genes, including $A B C$ transporters, $P D R 5, P D R 15$, and PDR16. It also confers increased resistance to cycloheximide (Hellauer, Akache et al. 2002). Nevertheless, deletion of RDR1 does not increase resistance to cycloheximide in cells lacking Pdr5p nor does it cause a change in expression of SNQ2. These results suggest that Rdr1p is a PDR5 suppressor and might act via the Pdr1p/Pdr3p binding site in front of the PDR5 promoter since insertion of a PDRE in front of a minimal CYC1 promoter increases PDRE-CYC1 reporter expression by 8 fold in $\Delta r d r 1$ cells compared to wild-type. Another explanation for rdr1 1 -induced PDR5 expression is that deletion of RDR1 causes damage in mitochondria which results in a phenotype similar to the one observed 
in $\rho^{0}$ cells, which then activate Pdr3p and therefore increases PDR5 expression indirectly (Moye-Rowley 2003). This explanation is supported by the fact that $\Delta r d r 1$ phenotype includes the inability of cells to grow on non-fermentable carbon sources as mentioned above. More studies are needed to understand how RDR1 regulates the $A B C$ efflux pump Pdr5p.

\section{MSN2 and MSN4}

Both Msn2p and Msn4p are $\mathrm{C}_{2} \mathrm{H}_{2}$ zinc finger transcription activators that are involved in general stress responses, such as heat shock, osmotic shock, oxidative stress, low $\mathrm{pH}$, glucose starvation, sorbic acid and high ethanol concentrations. Upon induction, they move from the cytoplasm to the nucleus and bind to stress response elements (STREs), 5'-CCCCT-3', located in the promoters of their target genes (Schmitt and McEntee 1996; Görner, Durchschlag et al. 1998). MSN4 gene expression is autoregulated, as well as being Msn2p-dependent, and is induced by stress, while MSN2 expression is constitutive (Görner, Durchschlag et al. 1998; Gasch, Spellman et al. 2000).

Fardeau et al 2006 had showed the contributions of Msn2p and Msn4p to PDR phenotype. Fardeau's group investigated early cellular responses that lead to the PDR phenotype finding six transcriptional regulatory motifs that significantly contribute the cellular response to fluphenazine, a PDR inducer. In fluphenazine induction, 15 induced genes contain Msn2p/Msn4p binding sites, including SNQ2. This suggested roles of Msn2p, and Msn4p in PDR response.

Consistent with this finding, Schuller et al 2007 found that perturbation of the plasma membrane with 2, 4-dichlorophenol (DCP), and polyoxyethylene-9laurylether (POELE) induced many changes in gene expression and a significant number of those genes contained binding sites for Pdr1p/Pdr3p, as well as Msn2/Msn4p. Further investigation showed that double mutation $\Delta m s n 2 \Delta m s n 4$ confers sensitivity in high concentrations of DCP, and that both DCP and POELE cause re-localisation of Msn2p-GFP from the cytoplasm to the nucleus. They suggested that Msn2p and Msn4p contribute to DCP resistance in a Pdr1p/Pdr3p dependent manner.

PDR15 encodes an $\mathrm{ABC}$ transporter involved in effluxing chloramphenicol, and is the closest homologue to Pdr5p. It contains Msn2p/ Msn4p binding sites and is 
regulated by both proteins, as well as by Pdr1p/Pdr3p. Although Pdr1p and Pdr3p modulate basal level of pPdr15p, under many stress conditions, such as osmotic stress and weak acid stress, PDR15 expression is induced by Msn2p but not Msn4p, and does not require Pdr1p/Pdr3p, nor the high osmolarity glycerol (HOG) MAPK signalling pathway, which normally tightly regulates Msn2p and Msn4p (Wolfger, Mamnun et al. 2004). These results show extensive complex crossreactions between the general stress response and PDR response.

\section{Basic Region-Leucine Zipper Transcriptional regulators (bZIP), Yap1p}

Another class of transcription factors that has been associated with the regulation of PDR is the basic region-leucine zipper (bZIP) family (Figure 1-12). The most studied bZIP transcription factor that associates with PDR phenotype is YAP1. The Yap1p transcription factor is required for oxidative stress tolerance (Moye-Rowley, Harshman et al. 1989). The Yap1p protein was purified based on the its biochemical similarity to mammalian AP1 which is also involved in regulation of oxidative stress (Harshman, Moye-Rowley et al. 1988). Yap1p binds to the yeast AP-1(yAP-1) recognition element (YRE) with the consensus sequence of TTACTAA differing only in the second base pair from the mammalian AP-1 site (Fernandes, Rodrigues-Pousada et al. 1997).

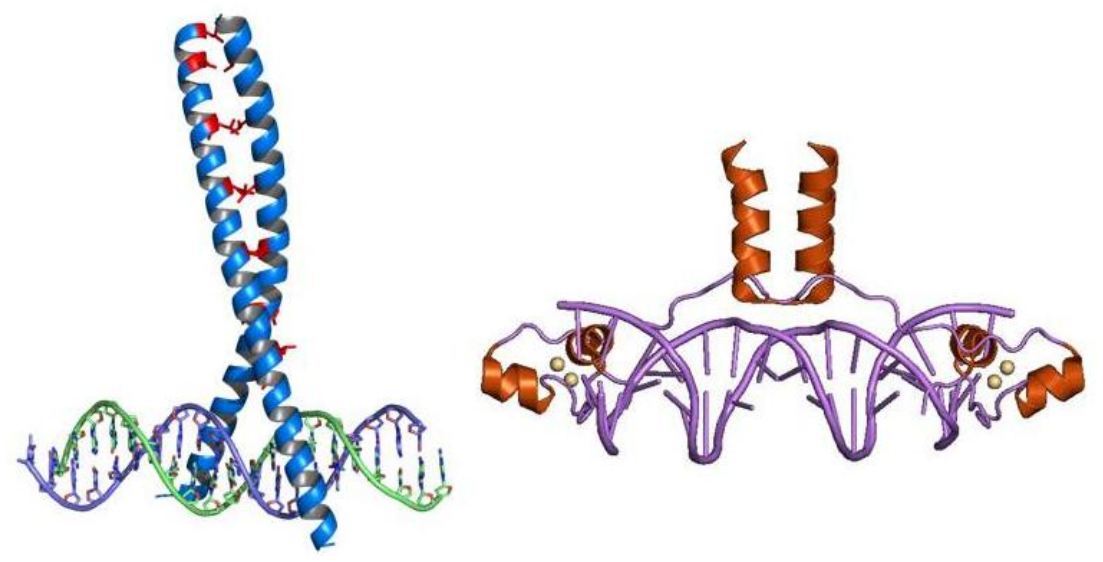

Figure 1-12: Structure of Basic Leucine Zipper and Zinc Cluster Transcription Factors.

The basic leucine zipper transcription factor (left). The leucine residues which comprise the zipper, are represented in red (image from http://en.wikipedia.org), and the Zinc Cluster Transcription Factor, Zn(II)2Cys6 (Right), is represented by yellow zinc atoms (image from MacPherson, S., M. Larochelle, et al. (2006)) 
YAP1 is activated by a change in cellular redox state caused by oxidants such as $\mathrm{H}_{2} \mathrm{O}_{2}$ and diamide through formation of disulfide bonds. The altered state causes re-localisation of Yap1p from the cytoplasm to the nucleus where it activates downstream target genes. Most of these genes encode radical scavengers such as glutathione (Gsh1p, Glr1p) or thioredoxin (Trx2p)(Jamieson 1998). It also mediates cadmium tolerance. More details about the Yap1p transcriptional activity mechanism will be discussed in later section "YAP1 and oxidative stress response" page 45 .

Interestingly, the first known biological phenotype associated with Yap1p was not in the oxidative stress response but it's ability to confer resistance to cycloheximide, sulfometuron, and 4-NQO when overexpressed (Moye-Rowley 2003). However, deletion of YAP1 does not cause hypersensitivity toward cycloheximide as does the deletion of $P D R 1 / P D R 3$ or ABC-transporter gene PDR5 (Wu, Wemmie et al. 1993). Wendler et al. showed that overexpression of YAP1 results in diazaborine resistance in a PDR1-and PDR3-dependent manner, thus linking the oxidative stress response with PDR. In contrast, overexpression of YAP1 can still confer resistance to cycloheximide in the $\Delta p d r 1 \Delta p d r 3$ background independently of PDR5 (Wendler, Bergler et al. 1997). As discussed above, many of the ABC transporter genes (PDR5, SNQ2) and major facilitators (ATR1, FLR1) contain YRE in their promoters and lie under the control of Yap1p. It is thought that cycloheximide tolerance might be achieved via regulation of $F L R 1$, and therefore bypasses the requirement for Pdr5p (Moye-Rowley 2003). Yap1p also activates Ycf1p, another ABC transporter that has the closest homology to Yor1p. Ycf1p has a role in detoxifying cells of metals such as cadmium, lead, and mercury, as well as oxidizing agents such as diamide ( $\mathrm{Li}$, Lu et al. 1997; Gueldry, Lazard et al. 2003). Ycf1p also involved in sequestering endogenous cellular toxins from metabolic processes (Paumi, Chuk et al. 2009), and act as one of the systems for exporting electrophiles that are conjugated to glutathione ( $\mathrm{Li}$, Szczypka et al. 1996).Therefore, activation of YCF1 by Yap1p could lead to drug resistance. The ability of Yap1 $p$ to activate MFS and other transporters, besides the ones activated by Pdr1p/Pdr3p, suggests that Yap1p acts on a parallel pathway to Pdr1p/ Pdr3p and functions to strengthen the PDR phenotype. Figure 1-13 shows some targets 
of Yap1p which contribute to the PDR phenotypes, including some that overlap with known PDR transcription factors, such as Pdr1p and Pdr3p.

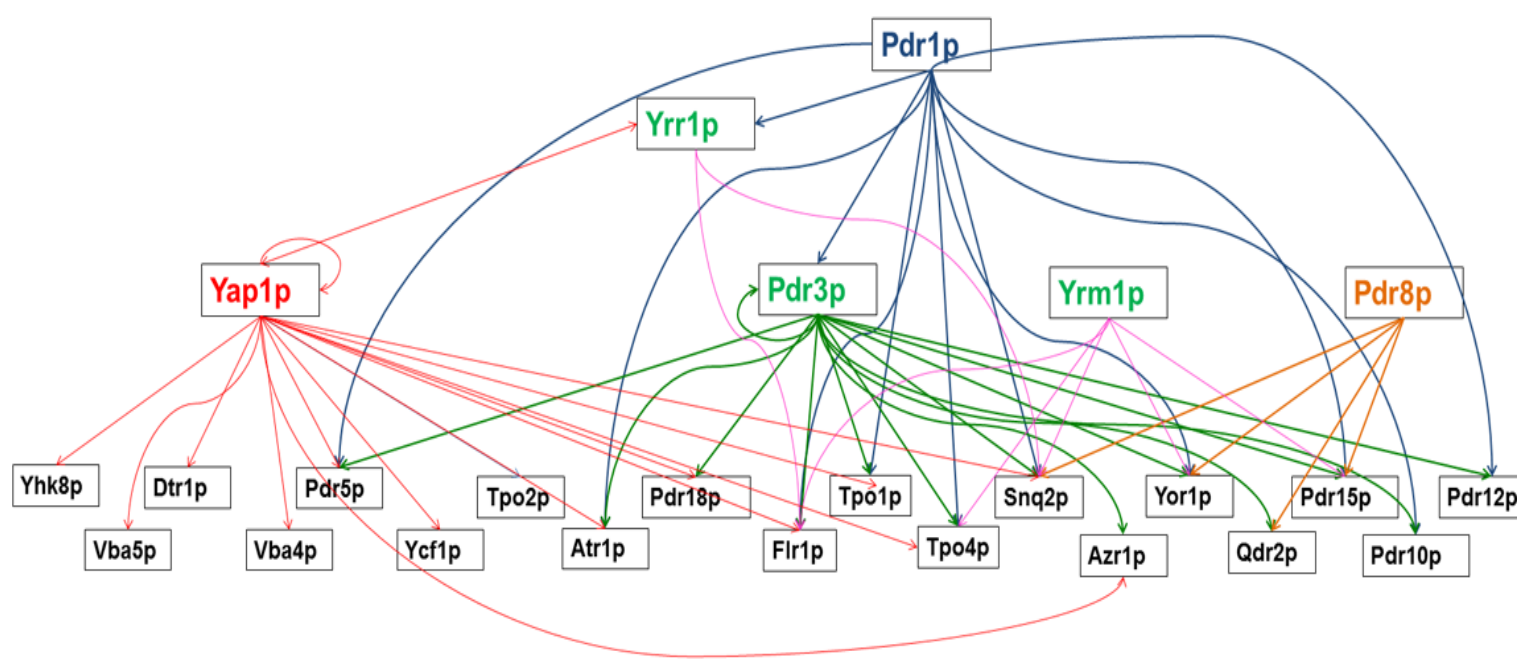

Figure 1-13: Yap1p target genes that are involved in PDR phenotypes.

\section{Other bZIP regulators}

Many close homologs of Yap1p in S. cerevisiae such as Cad1p and Cin5p show the ability to elevate tolerance to cadmium (Moye-Rowley 2003) and the chemotherapy drug cisplatin tolerance respectively (Furuchi, Ishikawa et al. 2001). As mentioned above PDR5 and SNQ2 expression can also be heat-shock activated by Yap1p as well as Cad2p (Miyahara, Hirata et al. 1996). Although Yap1p and Cad2p have been shown to be $88 \%$ identical in their DNA binding domain, Cad2 $p$ target genes are often involved in protein folding and degradation, while Yap1p target genes often show involvement in detoxification (Cohen, Pilpel et al. 2002). This property might allow cells to tolerate a larger variety of xenobiotic compounds.

Another class of bZIP regulators, called the activating transcription factor /cAMP response element binding protein (ATF/CREB) family, Aca1p and Aca2p, are involved in utilization of non-optimal carbon sources and chromosome stability. They have been shown to increase sensitivity toward many drugs including hygromycin B and cycloheximide when deleted (Garcia-Gimeno and Struhl 2000). 


\section{Other regulators of PDR}

\section{Mitochondrial status}

Screens aimed at identifying negative regulators of PDR5 show that cells carrying a deletion of $F Z O 1$ and/or OXA1 (genes involved in mitochondrial integrity) exhibit resistance to cycloheximide via a large increase in PDR5 expression. This occurs in a PDR3-, but not a PDR1-dependent manner (Hallstrom and Moye-Rowley 2000). FZO1 encodes an integral membrane protein involved in mitochondrial outer membrane tethering (by SNARES) and fusion, as well as having a role in mitochondrial genome maintenance (Rapaport, Brunner et al. 1998). Loss of $F Z O 1$ causes rapid loss of the mitochondrial genome ( $\rho^{0}$ cell) (Rapaport, Brunner et al. 1998). OXA1, on the other hand, encodes a mitochondrial inner membrane protein that interacts with mitochondrial ribosomes (Jia, Dienhart et al. 2003). Both $\rho^{0}$ and $\triangle o x a 1$ cells are defective in electron transport and oxidative phosphorylation and display an increased expression of PDR5 (Hallstrom and Moye-Rowley 2000).

Hallstrom and Moye-Rowley (2000) suggested that PDR3-dependent upregulation of $P D R 5$ and $P D R 3$ expression in $\rho^{0}$ cells occurs because PDR1 is constitutively expressed while $P D R 3$ is autoregulated. It follows that; $P D R 3$ may be regulated by Pdr1p via two PDREs in its promoter region. Cells that have lost their mitochondria genome $\left(\rho^{0}\right)$ have a 15-fold increase in PDR3 expression with no increase in $P D R 1$ expression (Zhang, Cui et al. 2001). The same authors showed that PDREs in the PDR3 promoter, and thus autoregulation property of $P D R 3$, are required for up-regulation of $P D R 5$ in the $\rho^{0}$ cells. The signal from the $\mathrm{F}_{0}$ subunit of the mitochondrial ATPase leads to PDR5 activation. Interestingly expression of SNQ2 and YOR1, two other targets of PDR3, do not increase as much in $\rho^{0}$ cells as $P D R 5$ does (Zhang, Cui et al. 2001).

Later experiments that screened for mutants, in which cycloheximide resistance in $\rho^{0}$ cells was abolished, identified a mutation in LGE1. Deletion of LGE1 causes a strong reduction of PDR3-dependent PDR5 expression in $\rho^{0}$ cells (Moye-Rowley 2005; Zhang, Kolaczkowska et al. 2005). Lge1p was required with other ubiquitin enzymes to attach ubiquitin to lysine in histoneH2B (Hwang, Venkatasubrahmanyam et al. 2003). It was demonstrated that PDR3 required Lge1p for normal induction in $\rho^{0}$ cells. Nevertheless, the actual mechanism of how 
Lge $1 p$ regulates $P d r 3 p$ in $\rho^{0}$ cells is still unknown. Lge1p has been linked with retrograde transport (Gulshan, Schmidt et al. 2008), and ubiquitination might play role in post translational modification of Pdr3p in $\rho^{0}$ cells.

Another possible link between mitochondrial status and PDR is a translocation of the phospholipid phosphatidylethanolamine (PE) from the inner leaflet of the plasma membrane bilayer to the outer leaflet stimulated by unknown "flippases" (Kean, Grant et al. 1997). PE biosynthesis is catalyzed by the enzyme phosphatidylserine decarboxylase (Psd1p) in the mitochondrial inter-membrane space. Experiments demonstrated that PSD1 in a high-copy-number plasmid confers an increase in cycloheximide tolerance in wild type cells via an increase in the PDR5 and PDR15 expression in a PDR3-dependent manner (Gulshan and Moye-Rowley 2007). Gulshan and Moye-Rowley also showed that loss of PSD1 in $\rho^{0}$ cells prevents normal activation of PDR5, and that the region in Psd1 $p$ required for this is in the non-catalytic amino-terminal of the protein (Gulshan and MoyeRowley 2007). Furthermore, Psd1p from other fungi, the human fungal pathogens, Candida albicans and Candida glabrate, also has the ability to activate cycloheximide resistance. This showed that Psd1p is also involved in regulation of multidrug resistance and is conserved throughout the fungal family.

Altogether, these findings show that mitochondrial status and regulation of the retrograde membrane flow pathway from the plasma membrane involves the multidrug resistance phenotype in S. cerevisiae, thus demonstrating the extent of diversity of the pathways affected by PDREs and their transcription factors.

\section{Lipid homeostasis}

As previously discussed, Pdr1p and Pdr3p control many ABC and MFS transporters that are plasma membrane proteins. Assuming that some transporters are flippases, it may be suggested that one major outcome of Pdr $1 \mathrm{p}$ and Pdr3p action is a change in plasma membrane composition, including "flipping" of PE to the outer-membrane bilayer leaflet (Decottignies, Kolaczkowski et al. 1994; Pomorski, Holthuis et al. 2004).

Lipid transporters establish a distinctly asymmetric lipid distribution between the outer membrane and inner membrane leaflet (Figure 1-14) and thus may affect the physiological function of the membrane. Many membrane-damaging agents can 
strongly induce $A B C$ pumps, demonstrating a coupling between lipid homeostasis and multidrug resistance (Pohl, Devaux et al. 2005; Sipo and Kuchler 2006).

Deletion of PDR5 and YOR1 decreases the phospholipid PE in the outer membrane leaflet of cells. Both Pdr5p and Yor1p translocate phospholipids across the membrane in an ATP-dependent manner (Decottignies, Grant et al. 1998). However, Pdr5p ATPase activity is much higher than that of Yor1p (Decottignies, Grant et al. 1998). The authors speculated that these differences in ATPase activity between these two $A B C$ transporters are possibly related to their differential activation by certain yeast phospholipids.

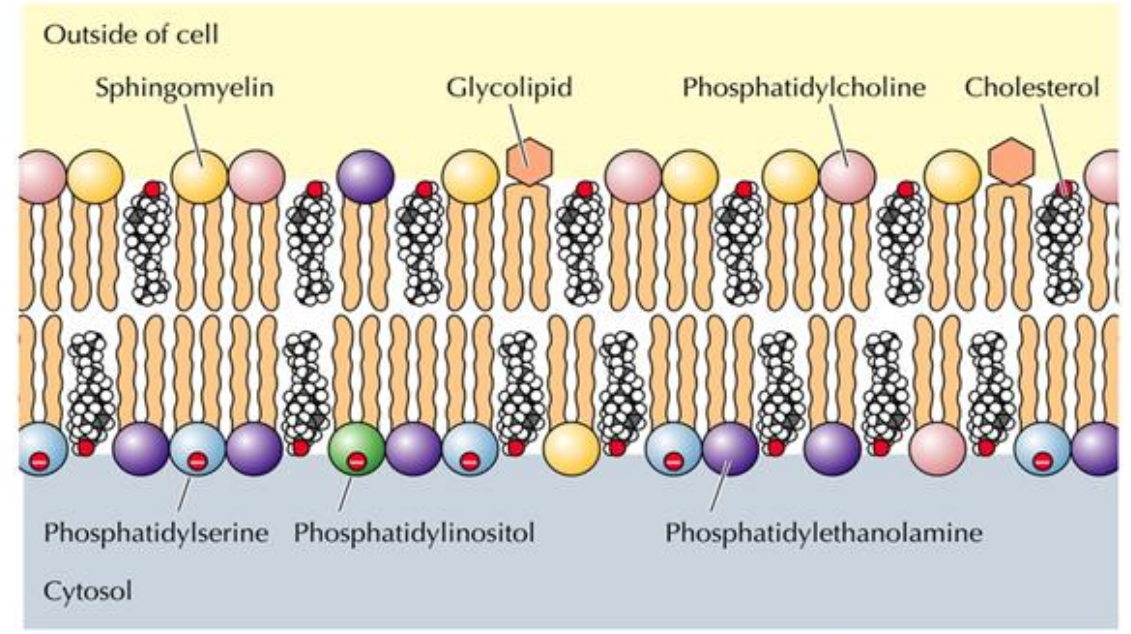

Figure 1-14: Composition of eukaryotic plasma membrane (courtesy ASM Press \& Sinauer Associates Inc, 2000)

The diagram shows that the plasma membrane comprises phospholipid bilayers in which specific phospholipids are found in one or other of the membrane leaflets.

It is also known that many eukaryotic $A B C$ transporters, such as yeast Pdr10 $p$ are found in lipid rafts (Pohl, Devaux et al. 2005; Sipo and Kuchler 2006). As mentioned above, PDR10 is a close homologue of PDR5, and is involved in maintaining the proper distribution of plasma membrane proteins. Deletion of this gene causes resistance toward weak organic acids due to accumulation of the $A B C$ transporter Pdr12p in the lipid raft. It might be expected, therefore, that flipping PE distribution could cause alteration in transporter function by affecting raft formation and retrograde membrane trafficking (Kihara and Igarashi .2004; Rockwell, Wolfger, et al. 2009). 
Mahe et al (1996) conducted experiments showing that certain ABC transporters have roles in both detoxification and transport of lipids and human steroids, which can also be toxic in certain forms. They demonstrated that overexpression of either PDR5 or SNQ2 can reduce cellular estradiol concentration in yeast cells containing an estradiol-inducible chimeric VP-16 human estrogen receptor. Deletion of both genes showed high level of estradiol accumulated in cells. Although the mammalian steroid hormone signaling pathway is not known in yeast, steroid derivatives play important roles in the yeast cell membrane, especially ergosterol, which is the main membrane sterol in yeast (Mahé, Lemoine et al. 1996).

Strains lacking Pdr5p and Yor1p are resistant to phytosphingosine (PHS), a precursor of many sphingolipids, involved in cellular signalling. Phytosphingosine induces apoptosis in human T-cell lymphoma and non-small cell lung cancer cells, and it induces caspase-independent cytochrome $C$ release from mitochondria. The PHS resistance is dependent on PDR1 and RSB1. Kihara and Igarashi suggested that there might be some signal transduction from Pdr1p to Rsb1p, which in turn alters the cell membrane sphingolipid composition (Kihara and Igarashi 2002). Close homologues of Rsb1p in Candida species, referred to as RTA, are also linked to multidrug resistance, as they are found to contribute to azole resistance in these pathogenic fungi (Jia, Ma et al. 2008).

The main phospholipids in $S$. cerevisiae are sphingolipids. IPT1 encodes inositolphosphotransferase, an enzyme responsible for the synthesis of mannose(inositol-P)2-ceramide $(\mathrm{M}(\mathrm{IP}) 2 \mathrm{C})$, the most abundant sphingolipid in the cell (Dickson and Lester 2002). IPT1 was found to be transcriptionally induced by Pdr1p and Pdr3p (Hallstrom, Lambert et al. 2001). Furthermore, mutation in IPT1 can confer resistance to the antifungal syringomycin $E$ (Thevissen, Cammue et al. 2000).

PDR16, and its closest homologue PDR17, which encodes phosphatidylinositol transfer protein (PITP) are found to be under the control of Pdr1p and influence levels of phospholipid and drug resistance (van den Hazel, Pichler et al. 1999). Deletion of PDR16 results in hypersensitivity to the azole antifungals miconazole and ketoconazole. Deletion of either gene, or both genes, causes change in sterol 
composition in the cell membrane, especially in the levels of lanosterol and zymosterol, as well as phospholipid composition (van den Hazel, Pichler et al. 1999). These results suggest that Pdr16p and Pdr17p contribute to multidrug resistance mainly via regulation of lipid composition rather than directly sequestering drugs, as do the other $\mathrm{ABC}$-transporter targets of Pdr1p.

Induction of phospholipid translocation across the membrane bilayer is also found in mammals. Mouse mdr1, mdr2 and human MDR1 and MDR3 act as phospholipid transporters or flippases (Decottignies, A., A. M. Grant, et al. (1998) and their references). MDR1 in the human adrenal cortex was suggested to be involved in transport steroid hormone and other lipids (Mahé, Lemoine et al. 1996). This shows that $A B C$-transporters, and their roles in phospholipid homeostasis, are conserved across species.

The review of the literature in the last few pages demonstrates fundamental effects of xenobiotics and disruption of the PDR systems on membrane composition (Sipo and Kuchler 2006) and trafficking. These effects devolve down to the molecular organization of membranes, not just the expression of the pumps themselves, as discussed in initial parts of this Introduction. What we do not know is whether this co-ordination of regulation affects just PDR pumps, as a special class of membrane proteins, or if it affects all membrane proteins.

Cells may also become resistant to toxic compounds by changing in the membrane ergosterol composition (Yibmantasiri, Leahy et al. 2012). Work by Emter et al 2002 showed that Erg6p an enzyme in the ergosterol biosynthesis pathway, determines the rate of passive drug diffusion, without affecting Pdr5p efflux pump activity in effluxing drug (Emter, Heese-Peck et al. 2002). This is a further hint as to the complexity of the PDR phenotype.

\section{HSP70}

Ssz1p (Pdr13p) is a member of the Hsp70 protein family. SSZ1 was first designated as PDR13 based on its ability to increase expression of both PDR5 and YOR1 when overexpressed, and therefore increase both cycloheximide and oligomycin tolerance (Hallstrom, Katzmann et al. 1998). Further investigation showed that Pdr13p acts via Pdr1p, but not Pdr3p, in inducing PDR5 and YOR1 expression. A dominant, gain-of-function mutation, S295F, in SSZ1 leads to 
resistance to cycloheximide and oligomycin and seems to be a more efficient activator of drug resistance than overexpression of the wild type allele (Hallstrom, Katzmann et al. 1998). Hallstrom et al (1998) suggested that Ssz1p regulates Pdr1p through post-translational phosphorylation, since mutations that change serine to phenyalanine suggest that phosphorlylation might be involved.

Another Hsp70 protein family member which seems to play a role in PDR regulation is Ssa1p as identified in a tandem TAP-tag affinity purification (Shahi, Gulshan et al. 2007). Ssa1p repressed PDR5 expression in a Pdr3p-dependent manner without having an effect on either Pdr1p-dependent gene expression or on other efflux pump targets of Pdr3p, i.e. Yor1p and Snq2p.

Thus, Hsp70 protein families provide another level of regulatory control of multidrug resistance in yeast emphasising the complexity of the PDR network.

\section{RNA polymerase II mediator complex}

The 'Mediator Complex' is a group of proteins that serve as "adaptors" between transcription factors and RNA polymerase. Mediators are essential components of the RNA polymerase II-mediated transcription machinery, a major point in gene expression regulation. Mediators consist of at least 20 subunits with multiple activities. They are conserved from yeast to humans, and play a role in both basal and regulated transcription (Casamassimi and Napoli 2007). Mediators can both activate and repress the expression of a particular gene, depending on which subunits are interacting with each other in the whole complex (Figure 1-15).

GAL11 (MED15) encodes a subunit of the RNA polymerase II mediator complex that is involved in multidrug resistance in yeast. Gal11p forms a complex with Pdr1p, via its activator-targeted KIX domain, and is required for drug resistance (Thakur, Arthanari et al. 2008). Deletion of GAL11 in both hyperactive mutants of $P D R 1$ and PDR3, although decreasing levels of PDR5 expression, does not completely abolish all the PDR5 expression from these strains. As with many of the strands of evidence discussed above, this literature suggests that there must be other mediators required for activation of multidrug resistance. 

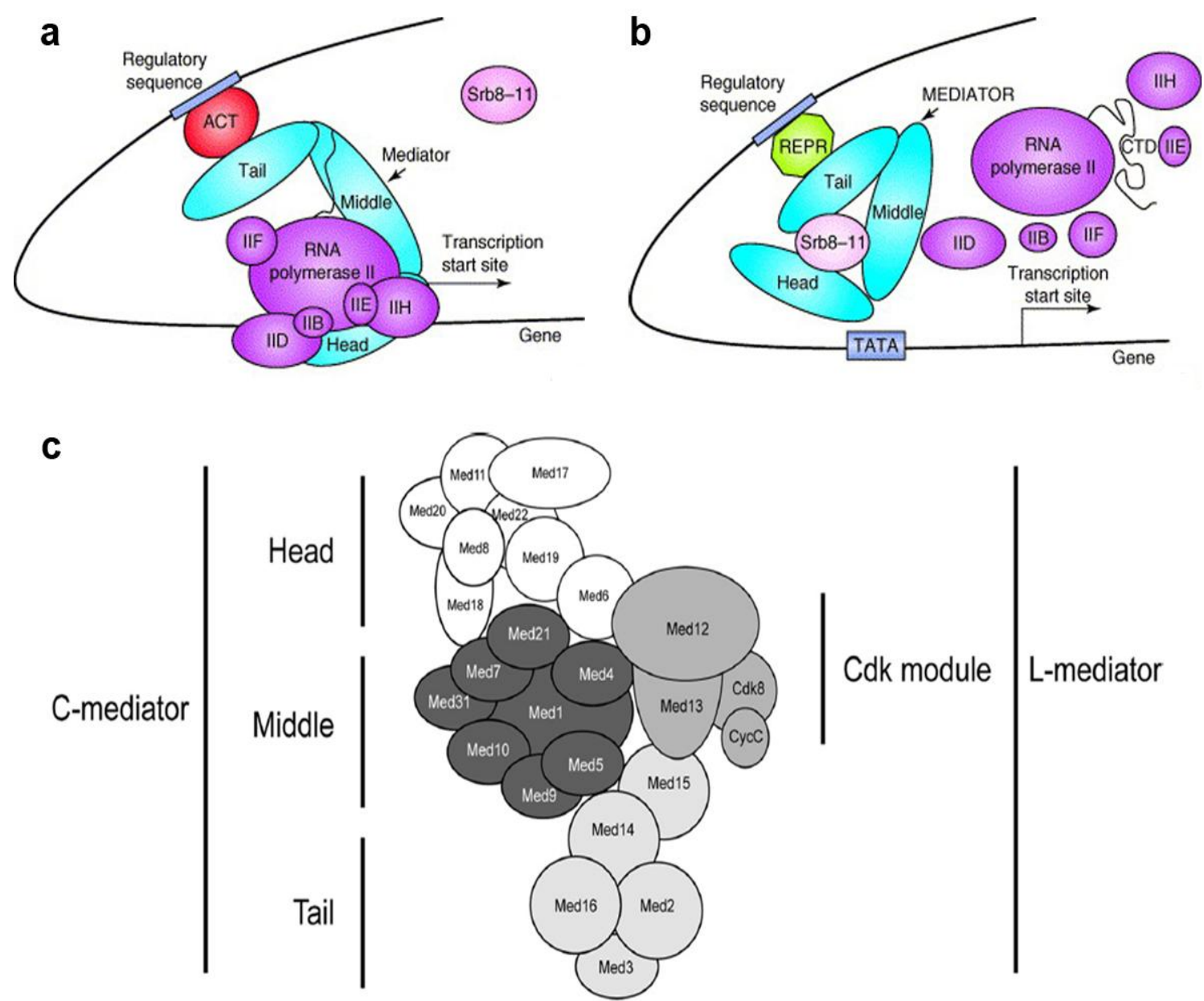

Figure 1-15: Yeast mediator complexes.

(a) The "Mediator" (blue) functions as a bridge between gene-specific activators (ACT; red) and the general pol II transcription machinery (purple) at the promoter". (b) "Gene-specific repressors (REPR; green) interact with specific Mediator subunits (blue) and recruit the complex to upstream regulatory DNA sequences. Mediator that is recruited by repressors contains the Srb8-11 (Med12-Med13-Cdk8-CycC) module (pink), which prevents interactions with Pol II and the basal transcription machinery (purple)". This figure and description is from (Björklund and Gustafsson 2005). (c) Yeast mediator components, figure from (Shahi, Gulshan et al. 2010).

Shahi et al 2010 investigated other possible mediators required for PDR3 dependent PDR5 activation in $\rho^{0}$ cells. They found that loss of the mediatorencoding gene SRB8 (referred to as MED12 in the paper) strongly reduced PDR5 induction and cycloheximide resistance in $\rho^{0}$ cells bur not in wt $\left(\rho^{+}\right)$cells. Further experiments by the same authors suggested that Srb8p plays an important role in mediating PDR3-dependent PDR5 activation, and does not interact with Pdr1p. In contrary, Gal11p is important for PDR5 induction, regardless of mitochondrial status, and interacts with both Pdr1p and Pdr3p (Shahi, Gulshan et al. 2010). 
Interestingly, a $\Delta p g d 1$ ( $\Delta$ med3) strain had a reduced $P D R 5$ expression in $\rho^{0}$ cells, as measured using a PDR5- $\beta$-galactosidase reporter, but did not change cycloheximide tolerance in the $\rho^{0}$ cells, while $\rho^{+} \Delta p g d 1$ cells are hypersensitive to cycloheximide. This might be due to different regulation/mediator requirements for cycloheximide tolerance in different genetic backgrounds. Each mediator subunit can also affect transcription of hundreds of genes and result in global alteration of gene expression. This in turn can result in specific PDR phenotypes, without directly regulating the major transcription factors Pdr1p/Pdr3p. Furthermore, disruption of each subunit in the same complex can result in different phenotypes. For example, loss of Srb8p and but not Ssn2p (Med13p), prevents the cycloheximide resistance phenotype in $\rho^{0}$ cells (Shahi, Gulshan et al. 2010).

A summarising diagram of the regulators/modulators that contribute to the PDR network and phenotype in yeast is shown in Figure 1-16.

\section{Mammalian multidrug resistance (MDR)}

Similarly to yeast, there are many ways cells, such as cancer cells, can become resistant to drugs, such as through the development of a block in the apoptosis pathway, or activation of detoxification systems involving cytochrome P450. There is evidence that activation of detoxification systems, such as DNA repair, can also induce expression multidrug transporters (Gottesman, Fojo et al. 2002). However, the most common way in which mammalian cells achieve multidrug resistance involves up-regulation of ATP-dependent efflux pumps, which is the same principle as that observed in yeast cells. Nevertheless, the exact mechanism of how cells sense the presence of drugs, and the possible signaling pathways that control transcriptional activation of $\mathrm{ABC}$-transporters, is still not clear. 


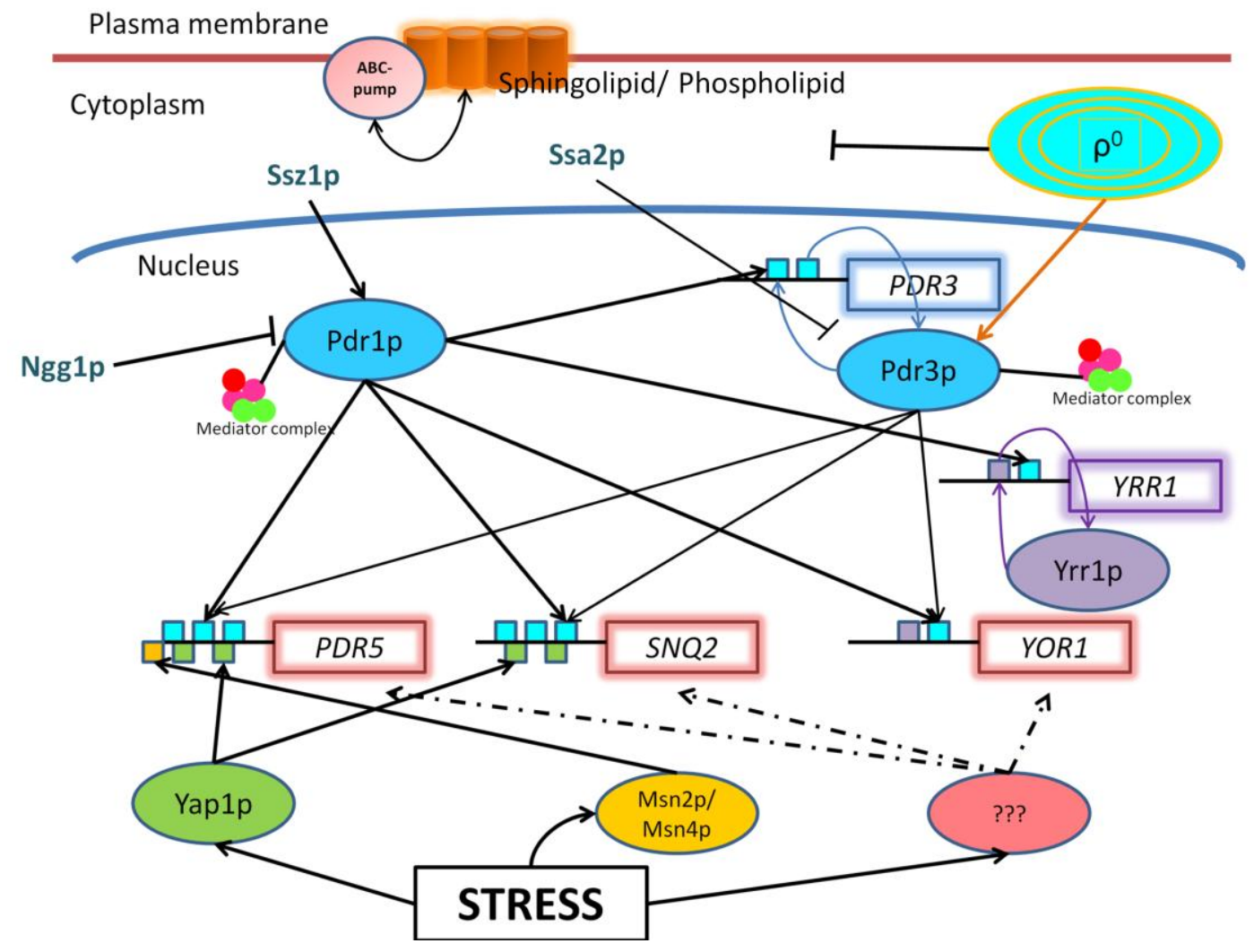

Figure 1-16: Diagram summarising of the regulators and modulators that contribute to the PDR network and phenotype in yeast.

Pdr1p and Pdr3p are the main transcription factors that activate (pointed arrow) the expression of ABC-transporter-encoding genes, such as PDR5 SNQ2 and YOR1, as well as autoregulating themselves. Other transcription factors, such as Yrr1p, Yap1p, and Msn2p, also contain recognition site(s) in the promoters of some of these ABC-transporter genes and help contribute to the PDR phenotype. Loss of mitochondrial DNA in cells $\left(\rho^{0}\right)$ activates Pdr3p, which in turn activates PDR5. Sphingolipids and phospholipids might also play roles in the PDR phenotype. Most ABC transporters are found in lipid rafts, and enhance outward movement of certain phospholipids. Some of the chaperone proteins (Ssz1p, Ssa2p) activate Pdr1p, while some inhibit its action (blunt arrowhead). There are likely to be more as yet unknown stress induced activators/ modulators, that could activate Pdr1p/Pdr3p, or activate $A B C$-transporter genes. Blue box: PDRE, Purple box: Yrr1p recognition site, Green: Yap1p recognition site (YRE), Yellow: Msn2p/Msn4p recognition site.

While multidrug resistance in yeast is often achieved via changes in transcriptional activity of $P D R 1$ and $P D R 3$, most multidrug resistance in mammalian cells seems to emerge via amplification of expression of $A B C$ transporter genes, which pump out drugs, and therefore lower cellular concentration of drugs (Gulshan and MoyeRowley 2007). The human ABCB1/MDR1, encoding the ABC transporter Pglycoprotein (Pgp), is overexpressed in certain tumour cells, leading to significant multidrug resistance to many chemotherapeutic drugs. Pgp is an efflux pump with 
a broad spectrum of substrates. Pgp has 12 transmembrane regions, and two ATP-binding sites. The transmembrane regions bind to drugs/substrates, which then stimulates the ATPase activity of the Pgp, which in turn results in conformational change that releases the drugs/substrates outside the cell. ABC transporters are found throughout the body in normal tissues and not just in cancer cells. In a different role, ABC transporters in the blood-brain barrier have a major role in protecting the brain against many toxins generated in the blood during metabolism. The liver, kidney, and gastrointestinal tract also use ABC transporters as a way to excrete toxins (Gottesman, Fojo et al. 2002). Many ABC pumps in mammals are used as a way to expose specific lipids on the outer membrane bilayer leaflet, for example as uptake receptors or to cause retrograde membrane transport to specific locations. As previously mentioned, in yeast these activities are mediated by MDR components acting as "flippases", which allow lipids to rapidly equilibrate between the two-layers and alter the biological properties of membranes (Pohl, Devaux et al. 2005).

Cancer cell lines, especially those from colon, kidney, adrenocortical, and hepatocellular cancers, were shown to highly express Pgp efflux pumps and exploit these transporters as mechanism to protect cancer cells from chemotherapeutic drugs (Gottesman, Fojo et al. 2002). Examples of drugs which are affected by up-regulation of efflux pumps are vinblastine, doxorubicin, daunorubicin, and paclitaxel (Gottesman, Fojo et al. 2002).

Since not all cancer cells exhibit high expression levels of Pgp pumps and because cancer cells are normally genetically heterogeneous due to accumulations of mutations, it is likely that other mechanisms might also be involved in multidrug resistance (Gottesman, Fojo et al. 2002). These include upregulation of other detoxification pathways, such as DNA repair as mentioned above, and mutations in the drug target. For example, many mutations are found in tubulin subunits, which form microtubules. These mutations prevent microtubule binding agents, such as the chemotherapeutic drug paclitaxol, from binding or interacting with microtubules, therefore making the cells become resistance to the drugs (Kavallaris 2010). Defects in the apoptosis pathways can also lead to drug resistance. Some cancer cells can reduce the levels of ceramide, which normally 
acts as signal for apoptosis, or some cancer cells are altered in their regulation of the cell cycle (Figure 1-17) (Gottesman, Fojo et al. 2002).

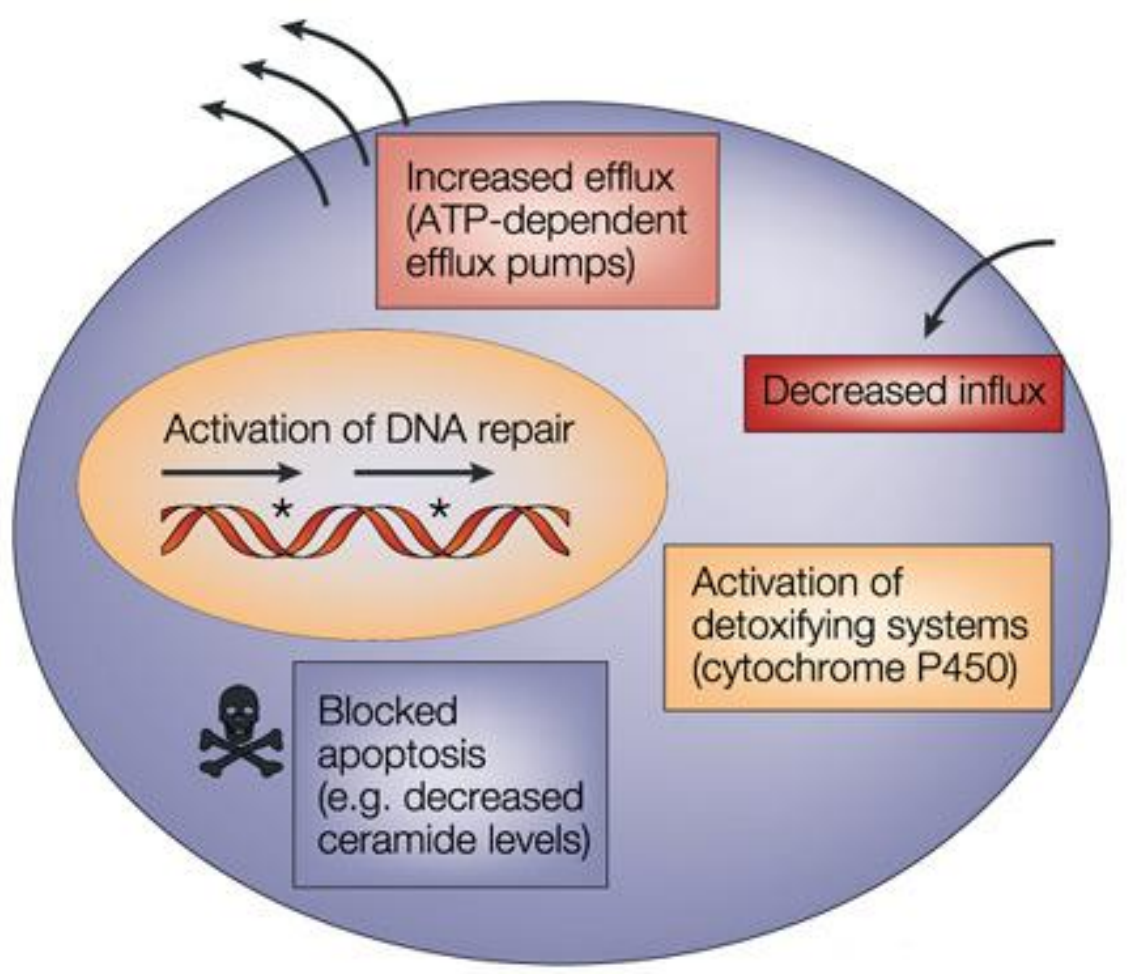

Figure 1-17: Mechanisms by which cancer cells achieve multidrug resistant phenotype.

Figure from (Gottesman, Fojo et al. 2002).

Although cancer cells use more than a single mechanism to develop resistance to a variety of drugs, and though the cytotoxic effects of many compounds may be exerted via physiological mechanism not present in yeast, many of the mechanisms underlying multidrug resistance are conserved from yeast to higher eukaryotes. Moreover, many of the $A B C$ transporters in yeast have functions analogous to Pgp pumps (e.g. Pdr5p and MDR1 Pgp), and Yor1p in yeast is related to the human MRP/CFTR family, as mentioned above. It is clear that a detailed knowledge of cellular mechanisms of multidrug resistance in yeast cells may contribute to a better understanding of the mechanism of multidrug resistance in mammalian cells. Particularly of how cells sense the presence of the drug, and how transcription factors that activate $A B C$ transporters are regulated, as well as answering some questions of how sphingolipids interact with $A B C$ transporters and contribute to the MDR response. All this information will greatly help in cancer 
treatment and might lead to the discovery of a new potential combination of drug targets-for example, one drug might help reduce the up-regulation of $A B C$ transporters, so that chemotherapeutic drugs will not get pumped out and remain at a concentration toxic to the cell.

\section{Questions concerning PDR and genomic tools}

Due to the complexity of the PDR network, many questions remain to be answered. For example, how do cells sense the presence of the xenobiotics? Pdr1p is found inside the nucleus and binds to the promoter of targets independent of the presence of drugs (Fardeau, Lelandais et al. 2007). Most of the PDR pumps (both $A B C$, and MFS) are kept at a low level during normal growth but are rapidly and highly induced in the presence of xenobiotics. How can exogenous compounds activate Pdr1p, which is found inside the nucleus in a rapid manner? Thaker et al 2008 suggested that Pdr1p/Pdr3p act as receptors and directly bind to xenobiotics (Thakur, Arthanari et al. 2008). This suggests that drugs must rapidly get through the plasma membrane to the nucleus to be able to bind directly to Pdr1p/Pdr3p to induce the rapid PDR response. So, how does such a wide variety of drugs get inside the cell? Some of the drugs are not lipophilic so they cannot diffuse across the lipid bilayers of the plasma membrane, and yet they trigger the PDR response. Are there any genes upstream of Pdr1p/Pdr3p which relay the information about the presence of xenobiotics to the nucleus? In addition, are there other unknown modulators that help regulate the transcriptional activity of Pdr1p/Pdr3p? (Figure 1-18).

As mentioned previously, there are many factors which can also contribute to the multidrug drug resistance phenotype, such as another transcription factor,Yap1p , a transcription factor for the oxidative stress response which, when overexpressed, confers resistance to many drugs and whose binding site is found in the promoter region of many of the $A B C$ transporters and major facilitators. Lipid homeostasis has also been a major contributor to the drug resistance phenotype. Many of the antifungal agents, such as amphotericin and nystatin (polyenes) are not $A B C$ transporter substrates and it has been shown that mutations in the ERG gene family, which is involved in sterol biosynthesis in $S$. cerevisiae, such as mutations in ERG2 and ERG3, confer resistance to these antifungal agents due to change in 
lipid/sterol homeostasis (Woods, Bard et al. 1974; Morrissey and Osbourn 1999; Balkis, Leidich et al. 2002; Emter, Heese-Peck et al. 2002).

Alteration in sterol composition also confers drug resistance in other fungal species, such as Candida albicans and Candida tropicalis (Hsuchen and Feingold 1974; Woods, Bard et al. 1974). Antifungal like ketoconazole, which is a Pdr5p substrate, can be less potent in cells which have a defect in sterol composition (Balkis, Leidich et al. 2002) suggesting that Pdr1p/Pdr3p despite being transcription factors with a major role in the multidrug resistance phenotype are not the sole contributors to PDR. It is very likely that many unknown regulators, modulators, transcription factors and lipid alterations also contribute to this phenotype. Cross talk between different responses are possible, and signaling cascades such as PKA, a signalling pathway known to be involved in general stress response, might also play an important role in relaying information about the presence of drug at the plasma membrane to the transcription factors Pdr1p/Pdr3p in the nucleus. If a signaling pathway such as PKA is involved, how can the cell distinguish between a general stress response and the drug resistance response? Is it possible that cells initially activate all the stress response pathways, and then distinguish them later through possible feedback loop? Alternatively, do cells use one pathway to strengthen another pathway's response? All these questions are still waiting to be answered. The more knowledge we gain about other pathways that contribute to the drug resistance network, the more we are equipped to answer these questions and try to overcome this problem in the future.

In the past, in order to study the regulators and modulators of Pdr1p/Pdr3p activity, one used classical genetics that involves screening for mutations that reverse certain phenotypes one by one, which is extremely arduous but may not always work for complex phenotypes. The traditional biochemical and genetic approaches can miss the pleiotropic nature of the function of many genes/proteins and can be affected by many factors, such as low affinity during protein-protein interaction purifications. The advantage of using yeast as a model organism is the availability of the genome-wide deletion set. This means that now we can design the screen in using a genome-wide approach and identify gene deletions that prevents the normal expression of reporter genes when cells are challenged with xenobiotics (the reporter genes here can be ABC transporters, which are Pdr1p/Pdr3p targets). 
Therefore, we can identify most of the non-essential genes that are involved in the modulation/regulation of the transcription factors Pdr1p/Pdr3p and therefore contribute to the PDR/MDR phenotype. We think that some of the genes which regulate or modulate the function of Pdr1p/Pdr3p might be non-essential, because most of the transcription factors involved in stress responses, including PDR, are non-essential, and many pathways of the yeast are redundant in optimal growth media (Zheng, Benschop et al. 2010; Hahn and Young 2011). Although we cannot rule out one of the essential genes being the "control switch" of the PDR response, our screen of non-essential genes is a good start for a relatively simple screening method using the deletion mutant array that became widely available since 2000 .

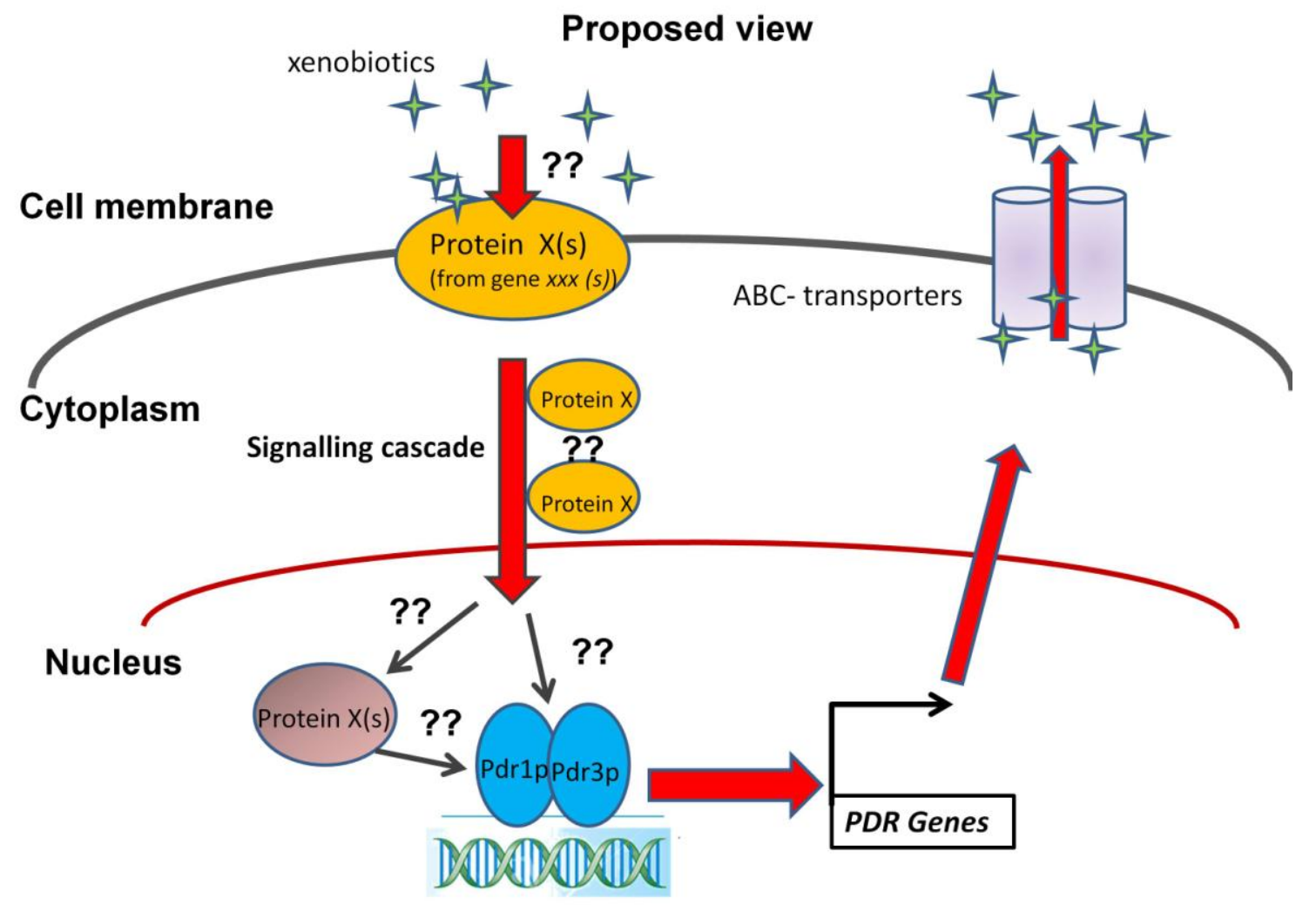

Figure 1-18: Proposed view of how cells sense xenobiotics

How cells sense the presence of the xenobiotic compounds and relay the information to the $\mathrm{dr} 1 / \mathrm{Pdr} 3 p$ in the nucleus. We propose that there is pathway (question mark in the figure) conducting drug information to Pdr1p and Pdr3p in the nucleus. 


\section{Use of a Yap1p-GFP reporter to investigate relationship between oxidative stress and PDR}

As mentioned above, expression of many of major drug transporter genes such as $P D R 5, S N Q 2$, and FLR1 can be induced by Yap1p, an oxidative stress response transcription factor. Much experimental evidence shows Yap1p contributes to the PDR phenotype (Wu, Wemmie et al. 1993; Alarco, Balan et al. 1997; Wendler, Bergler et al. 1997). However, numerous aspects of Yap1p regulation in oxidative stress are still unclear and the crosstalk if any between these two processes is unknown. Better understanding of Yap1p regulation during the oxidative stress response induced by different oxidants might also shed light on PDR.

\section{YAP1 and oxidative stress response}

Reactive oxygen species (ROS), such as the superoxide anion $\left(\mathrm{O}_{2}{ }^{-}\right)$, the hydroxyl free radical $\left(\mathrm{OH}^{\circ}\right)$ and hydrogen peroxide $\left(\mathrm{H}_{2} \mathrm{O}_{2}\right)$, are generated during oxygen metabolism, which causes oxidative stress. Some heavy metals (e.g. cadmium), UV radiation, and genotoxic agents can also generate oxidative stress causing damage to various cellular macromolecules, including DNA, lipids and proteins (Jamieson 1998). In general, cells maintain a particular redox state by utilising oxidative stress response systems. Similarly to the regulation of the multidrug resistance response, oxidative stress response regulation involves many transcription factors and modulators, the most well-known of which is a basic leucine zipper (bZIP) transcription factor called Yap1p (Moye-Rowley, Harshman et al. 1989). Yap1p functional orthologs have been identified in many other eukaryotes species such as S. pombe (Toda, Shimanuki et al. 1991), Arabidopsis thaliana (Babiychuk, Kushnir et al. 1995), C. glabrata (Chen, Miyazaki et al. 2007). Yap1p is one of the three conserved mechanisms involved in sensing oxidative stress and inducing anti-oxidant genes in S. cerevisiae, S. pombe and $C$. albicans (Ikner and Shiozaki 2005). The other two mechanisms are the stress-responsive MAPK (SAPK) cascade and the multistep phosphorelay system briefly discussed below (Figure 1-19) 

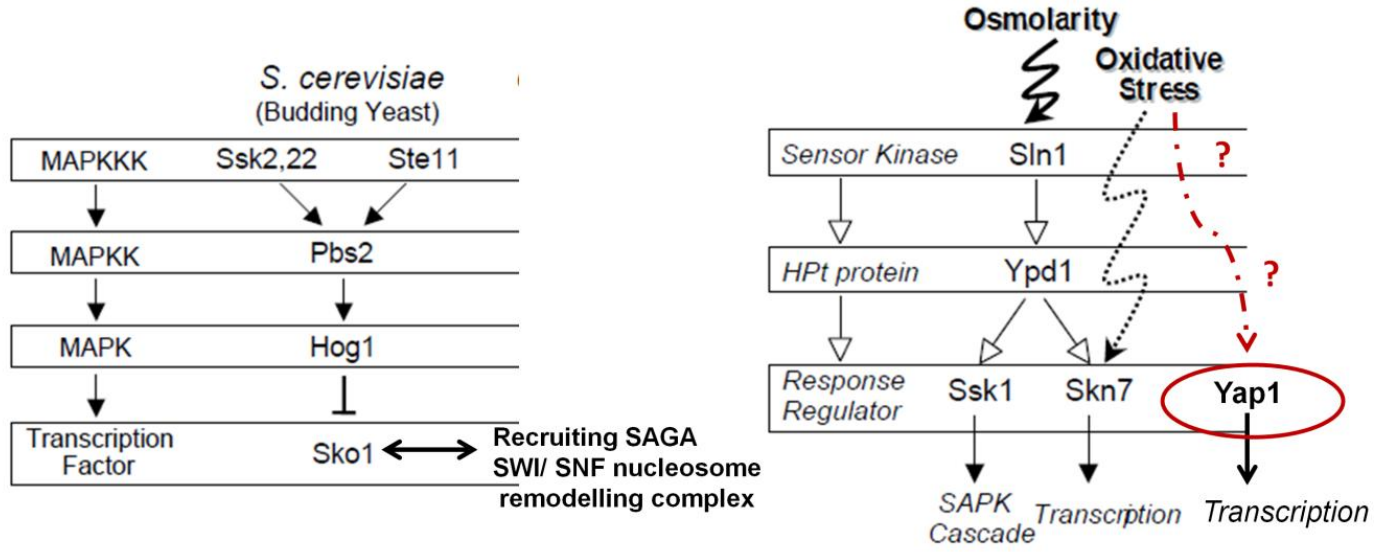

Figure 1-19: Different pathways of Oxidative stress response in yeast.

Figure modified from Ikner, A. and K. Shiozaki (2005)

The MAPKs are activated in response to stresses such as osmolarity, oxidative stress, heat shock and nutrient starvation (Ikner and Shiozaki 2005). The SAPK process involves a MAPK protein Hog1p, which transmits the stress signal and is well known in regulation of osmotic stress. Deletion of HOG1 confers sensitivity to both $\mathrm{H}_{2} \mathrm{O}_{2}$ and diamide. Upon osmotic stress, Hog1p enters the nucleus and phosphorylates Sko1p, which recruits the SAGA and SWI/SNF complexes. These complexes have roles in the chromatin remodelling and gene activation, but to date, there is no evidence that Hog $1 p$ MAPK regulates oxidative stress gene expression.

The multistep phosphorelay to sense oxidative stress involves Skn7p, which was first identified as a gene involved in cell wall biosynthesis and whose deletion caused hypersensitivity toward $\mathrm{H}_{2} \mathrm{O}_{2}$ in the genetic screen (Brown, North et al. 1993; Krems, Charizanis et al. 1996). Skn7p is a nuclear response regulator and transcription factor which regulates many anti-oxidant genes, such as TRX2 (thioredoxin), TSA1 (thioredoxin peroxidase), CTT1 (cytosolic catalase T; degrades hydrogen peroxide), TRR1 (thioredoxin reductase), and SOD1 (superoxide dismutase, which plays a role in oxygen radical detoxification and in copper ion buffering ) (Ikner and Shiozaki 2005). Some of these genes are also the target of Yap1p (TRX2, TRR1, SOD1). However, it is still unknown how oxidative stress signals are transmitted to Skn7p. 
Yap1p recognizes the sequence TTACTAA (Fernandes, Rodrigues-Pousada et al. 1997) in the 5' promoter sequence of its target genes, which are mainly antioxidant genes such as TRR1 (cytoplasmic thioredoxin reductase, which determines the redox state of the thioredoxin system), TRX2 (thioredoxin), GSH1 (rate limiting enzyme in glutathione biosynthesis), GLR1 (cytosolic and mitochondrial glutathione oxidoreductase, which converts oxidized glutathione to reduced glutathione,) and SOD1.

The Yap1p N-terminus contains the DNA-binding domain and nuclear localization signal (NLS), while the C-terminus contains a nuclear export signal (NES). Yap1p also contains two clusters of cysteine residues, referred to as the cysteine-rich domain in both $\mathrm{N}$-terminus ( $\mathrm{n}-\mathrm{CRD}$ ) and $\mathrm{C}$-terminus ( $\mathrm{c}-\mathrm{CRD}$ ). The $\mathrm{C}$-terminus contains three conserved cysteine residues Cys598 Cys620 and Cys629 (Coleman, Epping et al. 1999). Under normal growth conditions, Yap1p is found primarily in the cytoplasm due to dominant activity of the NES, and thus Yap1p which enters the nucleus is rapidly transported out by the exportin protein Crm1p, which binds to the Yap1p NES (Kuge, Jones et al. 1997; Yan, Lee et al. 1998). Yap1p and Crm1p interact in an oxidant-sensitive manner. When cells are in a normal oxidative state, Yap1p is reduced, and the interaction between Yap1p and Crm1p is strong. In response to oxidative stress such as $\mathrm{H}_{2} \mathrm{O}_{2}$, which results in a change in cellular redox status, a disulfide bond is formed between Yap1p Cys598 in the C-terminus and an oxidized cysteine residue of Gpx3p, thiol peroxidase. The Gpx3p-Yap1p disulfide bond then isomerises to one between Cys-598 on Yap1p C-terminus and Cys-3032 on the N-terminus of Yap1p (Delaunary, Pflieger et al. 2002). This alteration of the C-terminus is thought to mask the NES from Crm1p, thus disrupting the interaction between the two proteins and leading to the rapid re-localization of Yap1p to the nucleus, where it activates redox target genes (Moye-Rowley 2003; Ikner and Shiozaki 2005). The disulfide bond between the Cterminal cysteine residue and $\mathrm{N}$-terminal residues is not observed when the cell is challenged with a different oxidative stress inducer, diamide. It was found that during diamide treatment, multiple disulfide bridges were formed between cysteine residues in the C-terminus (Kuge, Arita et al. 2001).

This nuclear localization mechanism involves interaction between the NLS, NES and nuclear pore proteins and karyopherins (proteins involved in transporting 
molecules from the cytoplasm into the nucleus). It allows cells to rapidly recruit pre-existing proteins into an activated complex in the response to stress without having to synthesize them de novo (Moye-Rowley 2003).

\section{Mutation in the C-terminus and N-terminus of Yap1p disrupts Yap1p nuclear localisation}

Loss of the C-terminal cysteine-rich domain (C-CRD) or mutation in one of the conserved cysteine residues (C629A, S630A, and E631A) causes disruption in the NES, which results in Yap1p being constitutively localized to the nucleus. These mutants are resistant to diamide, but hypersensitive to $\mathrm{H}_{2} \mathrm{O}_{2}$, possibly because they fail to activate TRX2, a gene encoding the thioredoxin isoenzyme (Coleman, Epping et al. 1999).

Deleting different portions of the $\mathrm{N}$-terminal cysteine-rich domain result in constitutive Yap1p nuclear localization. Such systematic deletions pinpointed the sequences that are specific for regulation of response towards $\mathrm{H}_{2} \mathrm{O}_{2}$ and uncovered the fact that the $\mathrm{N}$-terminus is required for the normal function and regulation of Yap1p (Coleman, Epping et al. 1999).

\section{Other proteins involved in regulation of Yap1p}

Gpx3p also functions as a thiol peroxidase by mediating reduction of cellular $\mathrm{H}_{2} \mathrm{O}_{2}$ (Delaunay, Pflieger et al. 2002). Nevertheless, Delaunay et al 2002 showed that in a $\Delta g p \times 3$ strain, $10-30 \%$ of the cells in the population showed partial GFP-Yap1p ( $\mathrm{N}$-terminus tagged) staining in nucleus after 30 min of $\mathrm{H}_{2} \mathrm{O}_{2}$ treatment. The partial Yap1p nuclear localization in the $\Delta g p \times 3$ background suggested that other unknown mediators might play a role in Yap1p regulation.

TRX2 and TRR1 are targets of Yap1p, and are also important in recycling Yap1p and Gpx3p from the oxidized state to the normal reduced state once oxidative stress target genes are activated. As previously mentioned, Gpx3p is required to oxidize, and thus activate, Yap1p in the presence of $\mathrm{H}_{2} \mathrm{O}_{2}$ by disulfide bond formation. Gpx3p is directly oxidized by $\mathrm{H}_{2} \mathrm{O}_{2}$ and yields $\mathrm{H}_{2} \mathrm{O}$ and a sulfenic acid, Cys36-SOH, which in turn oxidizes Yap1p. In order to recycle oxidized Gpx3p and Yap1p, thioredoxin (mainly Trx1p andTrx2p) acts as electron donor and reduces both proteins (Delaunay, Pflieger et al. 2002). Delaunay et al 2002 proposed that reduction of Gpx3p by thioredoxin (Trx1p, Trx2p), with recycling of Gpx3p and 
Yap1p, provides cells with an efficient dual mechanism for hydroperoxide scavenging and regulation of Yap1p. Another protein that contributes to Yap1p regulations is Ybp1p. Veal et al. (2003) showed that Ybp1p acts in the same pathway as Gpx3p (a $\Delta g p x 3 \Delta y b p 1$ strain was no more sensitive to $\mathrm{H}_{2} \mathrm{O}_{2}$ than each of the single mutants). Ybp1p forms a stress-induced complex with Yap1p (even prior to $\mathrm{H}_{2} \mathrm{O}_{2}$ treatment), which influences the nuclear localization of Yap1p in the presence of $\mathrm{H}_{2} \mathrm{O}_{2}$, but not diamide. Furthermore, deletion of YBP1 results in increased diamide resistance (Veal, Ross et al. 2003). Ybp1p helps regulate the $\mathrm{H}_{2} \mathrm{O}_{2}$-induced expression of TRX2 and TRR1 in a Yap1p-dependent manner and is $\mathrm{H}_{2} \mathrm{O}_{2}$ specific. It might be that $\mathrm{H}_{2} \mathrm{O}_{2}$ stimulates the formation of the complex between Ybp1p and Yap1p and, in turn, Ybp1p helps stimulate the formation of disulfide bonds required for nuclear localization of Yap1p in response to $\mathrm{H}_{2} \mathrm{O}_{2}$ treatment (Veal, Ross et al. 2003). This information is summarised in Figure 1-20

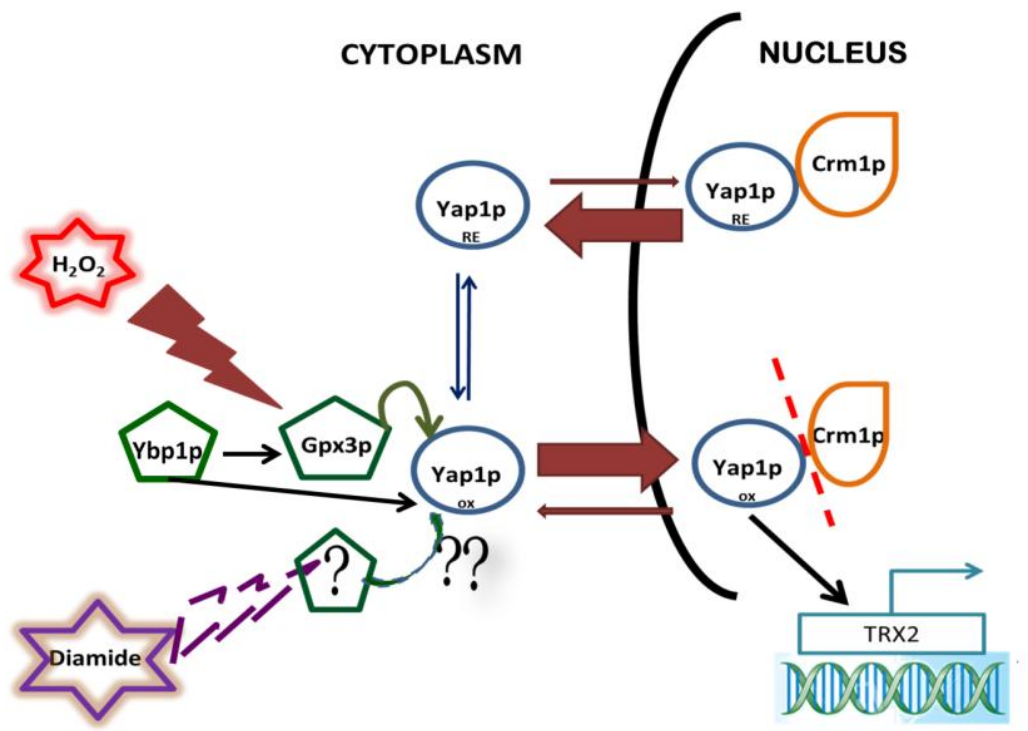

Figure 1-20: Model for regulation of Yap1p nuclear localisation.

In a normal oxidizing state, Yap1p is in a reduced state and interacts with Crm1p, which exports Yap1p back to the cytoplasm. Upon encountering oxidative stress induced by $\mathrm{H}_{2} \mathrm{O}_{2}$, Yap1p forms a complex with Ybp1p and Crm1p. Gpx3p induces disulfide bond formation between $\mathrm{n}$ - and $\mathrm{c}-\mathrm{CRD}$. This leads to a conformational change and prevents interaction between Yap1p and Crm1p. As a result, Yap1p is relocalised to the nucleus, where it activates redox target genes such as TRX2. Although proteins involved in Yap1p disulfide formation upon $\mathrm{H}_{2} \mathrm{O}_{2}$ encounter are known, less is known about proteins involve in C-CRD disulfide formation during treatment with diamide and other oxidants. Diagram adapted from Veal, Ross et al. (2003). 


\section{Mediator components}

Rox3p (Med19p) is a subunit of the RNA polymerase II mediator complex and it has been found that loss of $R O X 3$ results in a dramatic decrease in $\mathrm{H}_{2} \mathrm{O}_{2}$ tolerance while deletion of other mediator subunits such as GAL11 (mediator of PDR1 $P D R 3$ ) , MED2, MED1, or MED9 do not result in any change in $\mathrm{H}_{2} \mathrm{O}_{2}$ tolerance (Gulshan, Rovinsky et al. 2005). Nevertheless, loss of YAP1 has a more profound effect on the $\mathrm{H}_{2} \mathrm{O}_{2}$ tolerance than loss of $R O X 3$, and therefore YAP1 expression, to a certain extent, bypasses the requirement of Rox3p. The increase in sensitivity toward $\mathrm{H}_{2} \mathrm{O}_{2}$ in $\triangle$ rox 3 cells was shown to be the result of a loss of Yap1p induction by $\mathrm{H}_{2} \mathrm{O}_{2}$ in this strain, which results in reduced levels of TRX2 (Gulshan, Rovinsky et al. 2005). Gulshan $\mathrm{K}$ et al. also showed that correct folding of Yap1p in an $\mathrm{H}_{2} \mathrm{O}_{2^{-}}$ treated environment is required for the binding between Rox3p and Yap1p. He suggests that Rox3p might also be involved in receiving a redox signalling.

\section{Other transcription factors that regulate oxidative stress response}

Similar to the PDR response, the oxidative stress response is also thought to be modulated by many transcription factors, some which are also involved in the PDR response such as Msn2p/Msn4p. We will discuss these transcription factors in detail below.

\section{ACE1}

Copper and metals such as iron have the ability to transfer and gain electrons from ROS, which makes these metals potentially toxic. Therefore, activity of free copper and iron is tightly controlled in cells as a way to defend themselves against oxidative damage in addition to ROS detoxification (De Freitas, Wintz et al. 2003). The copper-binding transcription factor Ace1p regulates genes such as CUP1, which encodes the antioxidant metallothionein, and SOD1, which encodes a cytosolic copper-zinc superoxide, which plays a role in ROS detoxification and copper ion buffering. Although, the exact role of Ace1p in oxidative stress regulation is unclear, it is thought that Ace1p might act as an oxidant sensor (Jamieson 1998).

\section{HAP1}

The zinc-finger transcription factor Hap1p is involved in the complex regulation of gene expression in response to levels of oxygen and heme, as well as regulating 
SOD1, CTT1, and CTA1 (all of which play a role in ROS detoxification). The last step of heme biosynthesis is the incorporation of iron into porphyrin, which occurs in mitochondria. This results in influx of free iron into mitochondria, and generation of free radicals, thus causing mitochondrial oxidative stress. Cta1p is catalase A, which breaks down hydrogen peroxide, found in both peroxisome and mitochondria matrix. Nevertheless, how much Hap1p contributes in oxidative stress regulation is less clear. The Hap1p recognition sequence is found in the YAP1 promoter (Harbison, Gordon et al. 2004), but strains containing a mutation in $\mathrm{HAP} 1$ still possess the ability to adapt to $\mathrm{H}_{2} \mathrm{O}_{2}$-induced oxidative stress (Jamieson 1998; De Freitas, Wintz et al. 2003).

\section{MSN2, and MSN4}

As mentioned previously, the Msn2p/Msn4p zinc-finger transcription factors are activated under stress conditions and regulate general stress response (MartínezPastor, Marchler et al. 1996; Gasch, Spellman et al. 2000; Causton, Ren et al. 2001). Msn2p/Msn4p are activated by phosphorylation by Hog1p, which is a protein in the SAPK-cascade thought to link to the oxidative stress response, as mentioned previously. In response to oxidative stress, Msn2p and Msn4p regulate the expression of CTT1 (which encodes cytosolic catalase T). Similarly, to Yap1p, Ms2p and Msn4p localize to the nucleus in response to stress where they activate transcription of target genes.

\section{STB5}

In the presence of $\mathrm{H}_{2} \mathrm{O}_{2}$, Stb5p, a zinc cluster protein, binds and regulates the expression of many genes in the pentose phosphate pathway, as well as genes involved in the production of NADPH, a metabolite required for oxidative stress tolerance. NADPH is used in the thioredoxin pathway to convert $\mathrm{H}_{2} \mathrm{O}_{2}$ to $\mathrm{O}_{2}$ and $\mathrm{H}_{2} \mathrm{O}$ as well as acting as the eletron donor of reducing equivalents to the oxidized glutathione via glutathione reductase. Deletion of STB5 leads to diamide and $\mathrm{H}_{2} \mathrm{O}_{2}$ sensitivity, and following the addition of diamide, Stb5p binding increases at some of its targets' promoters (Larochelle, Drouin et al. 2006). Interestingly, activation of Stb5p does not seem to be regulated by the MAP kinase pathway or a mechanism similar to Yap1p. This suggests that there might be other unknown modulators that provide an alternative pathway for both the oxidative stress response and the multidrug resistance response, both of which rely on Stb5p. 
Many of the stress transcription factors mentioned above (Hap1p, Msn2p, Skn7p, Stb5p) have recognise elements in the YAP1 promoter (Lee, Rinaldi et al. 2002; Harbison, Gordon et al. 2004; Ni, Bruce et al. 2009). However, little is known about the interplay among transcription factors or about regulators other than Yap1p that may play a role in this stress regulation.

\section{Questions on Cellular Redox Regulation}

: Do redox-sensing proteins have the ability to discriminate between different oxidative stresses?

: Do these proteins produce distinct outputs which lead to the cells responding to a specific stress?

: Do different stress-inducing agents modify the same sensing/ regulator proteins to give different outcomes, or do different stresses use different sensing/regulator proteins?

A diagram representing the possible regulation of the oxidative stress response in S. cerevisiae is shown in Figure 1-21.

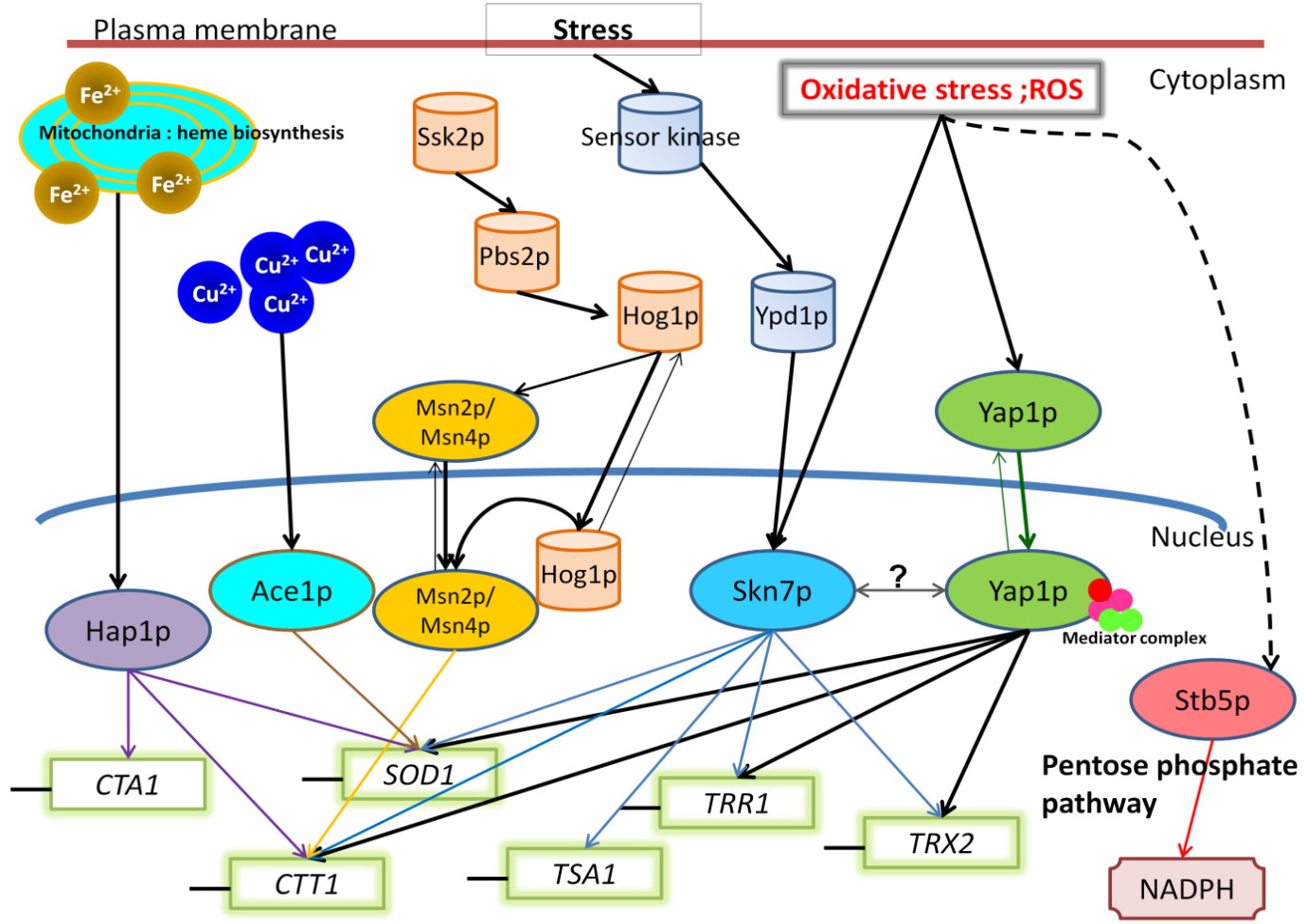

Figure 1-21: Schematic representation of oxidative stress response regulation in yeast 


\section{Yap1p and regulation of the multidrug resistance response}

\section{Overexpression of YAP1}

The role of YAP1 in the multidrug resistance phenotype was identified in a screen that searched for genes that gave rise to multidrug resistance when expressed from a high-copy plasmid (Moye-Rowley, Harshman et al. 1989; Hertle, Haase et al. 1991). The Yap1p binding site was found in the promoters of many of the transporters involved in the PDR, such as PDR5, SNQ2,YCF1, and FLR1 (Kolaczkowska and Goffeau 1999), although PDR5 and SNQ2 expression in a $\triangle y a p 1$ background was similar to that in wild-type (Miyahara, Hirata et al. 1996). When the $\triangle$ yap 1 cells were exposed to heat shock at $37^{\circ} \mathrm{C}$, however, the expression level of $P D R 5$ and SNQ2 was dramatically reduced in comparison to that in the wild-type cells (Miyahara, Hirata et al. 1996), suggesting that PDR5 and SNQ2 respond to heat shock stress in a Yap1p-dependent manner. Overexpression of YAP1 confers resistance to cycloheximide, which is thought to be the result of increase in the expression of FLR1, because the resistant phenotype is also observed in a background strain lacking PDR5 (Moye-Rowley 2003). Although expression of PDR5 and SNQ2, the two major ABC transporters, respond to Yap1p transcriptional activity, the disruption of these two genes had no effect on oxidative stress response. Pdr5p and Snq2p might have extra roles in other stress responses via activation by Yap1p.

It is not certain if Yap1p works in the same pathway as Pdr1p/Pdr3p when it comes to regulation of multidrug resistance. Deletion of YAP1 does not confer extra sensitivity toward cycloheximide in a $\Delta p d r 1 \Delta p d r 3$ background strain (Namal Coorey, personal communication). It is thought that Yap1p might be used as the "signal amplifier" of Pdr1p/Pdr3p. This is supported by the fact that YAP1mediated resistance to diazaborine and 4-NQO is PDR1 and PDR3-dependent, with a greater dependency on PDR3 (Wendler, Bergler et al. 1997). Nevertheless, the Pdr1p/Pdr3p recognition sequence (PDRE) is not found in the YAP1 promoter region (www.yeasttract.com). However, 1,10-phenanthroline hyper-resistance was observed only when YAP1 was overexpressed and the phenotype was stronger than the one observed in the gain-of-function mutant of PDR1-12 and PDR3-33, suggesting that Yap1p might also have a role which is Pdr1p/Pdr3p-independent (Wendler, Bergler et al. 1997). It is interesting to note that neither overexpression 
of Yap1p target genes, which are normally involved in the oxidative stress response, such as GSH1 GLR1 and TRX2, nor deletion of YAP1 ( $\triangle$ yap1) conferred resistance to diazaborine (Wendler, Bergler et al. 1997), implying that Yap1p might have a distinct role in drug resistance which is separate from its wellknown role in oxidative stress regulation. Wendler et al. suggested that a possible explanation for the fact that deletion of YAP1 did not cause extra sensitivity to diazaborine in the $\triangle p d r 1 \Delta p d r 3$ background might be that $Y A P 1$ acts on the same targets, or that $Y A P 1$ is acting on the same sequential pathway as PDR1, PDR3. These transcription factors might also share common regulatory proteins, which would normally suppress the function of their target proteins, and when YAP1 was overexpressed, these regulatory proteins bind mostly to Yap1p, leaving Pdr1p and Pdr3p free to activate target genes. As we know, Yap1p has many targets which overlap with Pdr1p and Pdr3p, such as Pdr5p and Snq2p, but Yap1p displays a different level of resistance to difference toxic compounds when YAP1 is overexpressed (e.g. diazaborine, cycloheximide and 1,10-phananthroline), suggesting that another unknown regulator might be involved.

Although there is a large body of experimental evidence indicating that YAP1 contributes to the multidrug resistance phenotype, the actual sensors or modulators that linked between Yap1p in oxidative stress response and Pdr1p Pdr3p in the pleiotropic drug resistant response are yet to be established. In addition, how Yap1p might distinguish its redox gene targets from PDR targets is unknown. 


\section{Section IV: Aim, hypothesis and experimental design}

The overall aim of my research was to understand complex traits through genetic network analysis. Such traits include drug resistance and most drug mechanisms.

There were three main questions in my project:

- Can we determine a drug target and mechanism of action by genome-wide deletion array analysis assuming a drug can act in lieu of a mutation?

- Are there upstream regulators (to the PDR TFs) that mediate the PDR response?

- Are such "sensors" or "stress regulators" stress specific? Or are they pleiotropic?

The aim of my project was to develop genomic methodologies to study drug mechanisms of actions and resistance mechanisms, and in the latter, specifically genes involved in regulation of pleiotropic drug resistance (PDR) signaling pathways that lie "upstream" of the PDRE-activated gene expression of pumps. It was aimed to identify regulators of Yap1p and the possible "link" between oxidative stress response and PDR.

In the case of PDR/MDR it is implicit in this aim that we planned to test whether there is a specific pathway of events that conducts information from the cell surface to the nucleus where Pdr1p and Pdr3p are located quite probably including internal signalling receptors.

The thesis is aimed at the question of whether an unbiased genome-wide of genetic interaction networks can identify previously unsuspected regulators of how cells deal with xenobiotics.

\section{Overall experimental design}

\section{Mode-of-action of toxic compounds using a genome-wide approach for network analysis}

Determining the compounds/drugs mode-of-action remains a major challenge in drug discovery. Genetic interaction networks illustrate sets of compensating pathways thus understanding which genes compensate for compound toxicity 
should give us insight as to how toxic compounds work, the possible side effects as well as mechanisms of resistance.

Many xenobiotics are neither substrates of the ABC transporters, nor MFS transporters, and are not pumped out of the cells in a Pdr1p/Pdr3p -dependent manner. The well-known antifungals, amphotericin B and nystatin fall into this category (Figure 1-22). Both of these antifungals bind to the ergosterol and disrupt the membrane integrity, presumably by forming pores and causing cellular leakage of internal constituents, which leads to cell death (Bossche, Engelen et al. 2003). Interestingly, cells that become resistant to both compounds have mutations in either ERG2 or ERG3, both of which play roles in ergosterol biosynthesis. The fact that sterol composition of the resistant strains also changed (Bossche, Engelen et al. 2003) strongly suggested that lipid homeostasis has a significant impact on the PDR phenotype. Although much of the thesis aims revolve around the modulators and regulators of the transcription factors Pdr1p/Pdr3p, and, to some extent, those of Yap1p, we will briefly explore the role of lipids as contributors to the PDR phenotype.
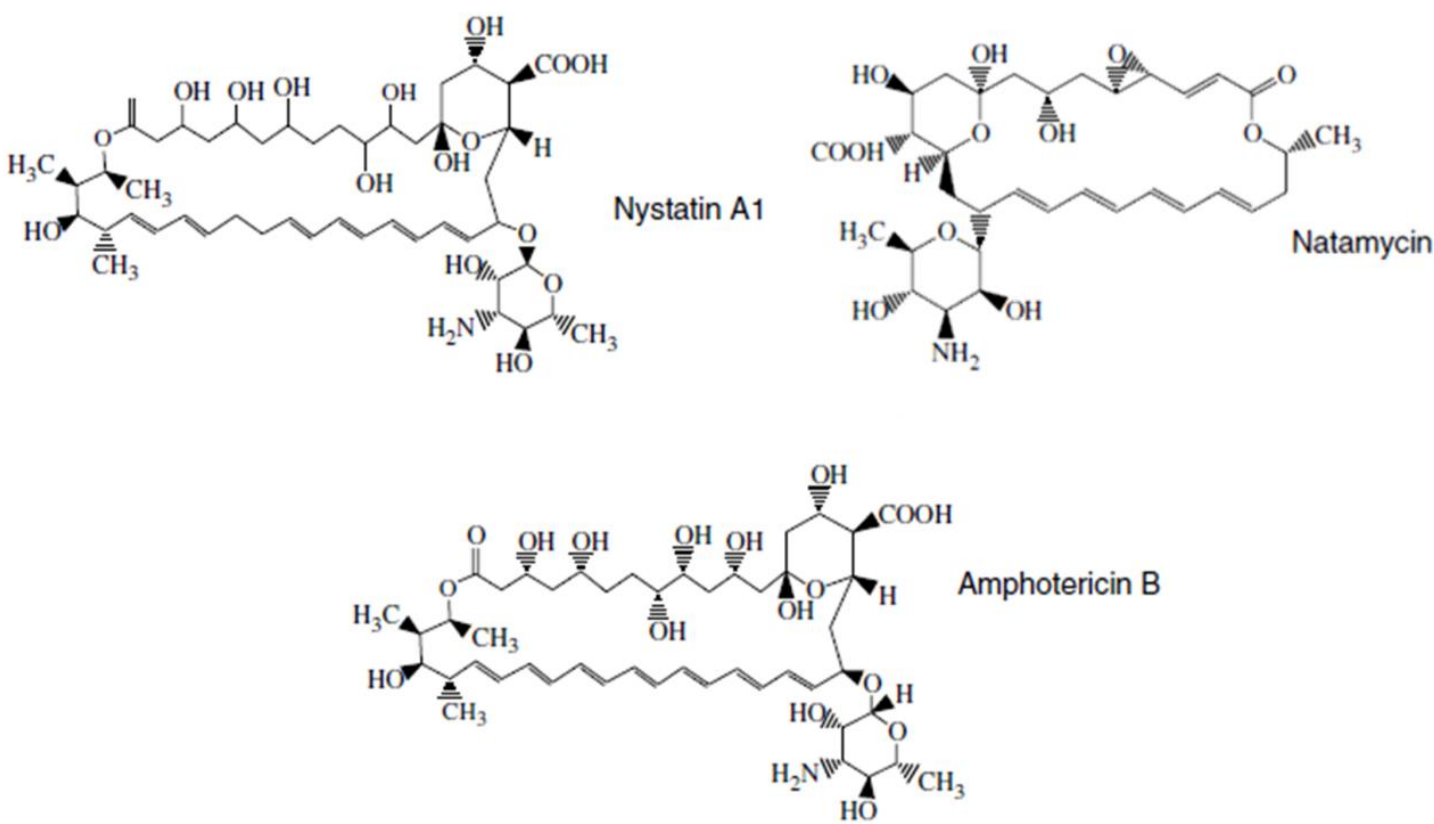

Figure 1-22: Structure of known polyene antifungal drugs.

Structures of the known polyene antifungals, nystatin, and amphotericin $B$, and the antifungal natamycin. None of these compounds are of ABC or MFS transporters. 
In this regard, we studied the mode of action of the novel compound neothyonidioside, from Australostichopus mollis. Neothyonidioside (Neo) has been shown to bind to ergosterol and is not a substrate of $A B C$ pumps. The gene which gives rise to neo resistance is NCP1, encoding NADP-cytochrome P450 reductase, which interacts with ERG11 and is involved in ergosterol biosynthesis. Cellular ergosterol levels of the neo-resistant cells are half of what is observed in normal wild-type cells (Yibmantasiri, Leahy et al. 2012). Neo also does not perforate the cell membrane and cause cellular leakage, like the classic antifungal amphotericin. Since $\mathrm{Neo}$ is not an $\mathrm{ABC}$-transporter substrate, nor does it behave like classic polyenes, it is of interest to us to explore how neo exerts its cytotoxic effect, what pathway might be involved and how affecting lipid homeostasis can lead to the drug resistant phenotype in a Pdr1p/Pdr3p-independent pathway.

\section{Identification of upstream regulators of PDR transcription factors Pdr1p/Pdr3p}

To identify modulators of the PDR response which regulate Pdr1p/Pdr3p in a genome-wide (genomic) fashion, we developed a Green Fluorescent Protein (GFP)-based reporter fused to Pdr5p, Snq2p, and Yor1p, as a subset of important $A B C$ transporters that are targets of Pdr1p/Pdr3p. The current literature does not have such an approach, probably because the automated microscope needed for these screens has only recently been feasible for academic laboratories. Such assays allow GFP fluorescence to serve as a sensitive, real-time indicator of Pdr1p/Pdr3p activation. Utilising high-throughput (genomic) mating techniques, our GFP-reporters were placed into the yeast gene deletion background, creating a $\sim 4800$ GFP-strain collection for each reporter. These new strains were then challenged with xenobiotics that in the "wild-type" would upregulate the efflux pumps. We then measured the efflux pump reporter fluorescence imaged by highthroughput confocal microscopy, and analysed it by specialized scripts developed for the work. This approach allowed for comparison of the changes between the control and treated experiment for each of the deletion strains. We then looked for genes that are required for PDR induction. These genes, when absent, cause changes in GFP-pump abundance, which should imply that these genes are required for normal pleiotropic drug resistance involving PDR1 and PDR3 and are potentially involved in PDR signaling upstream of Pdr1p/Pdr3p (Figure 1-23). 


\section{The Reporters: PDR5_GFP fusion}

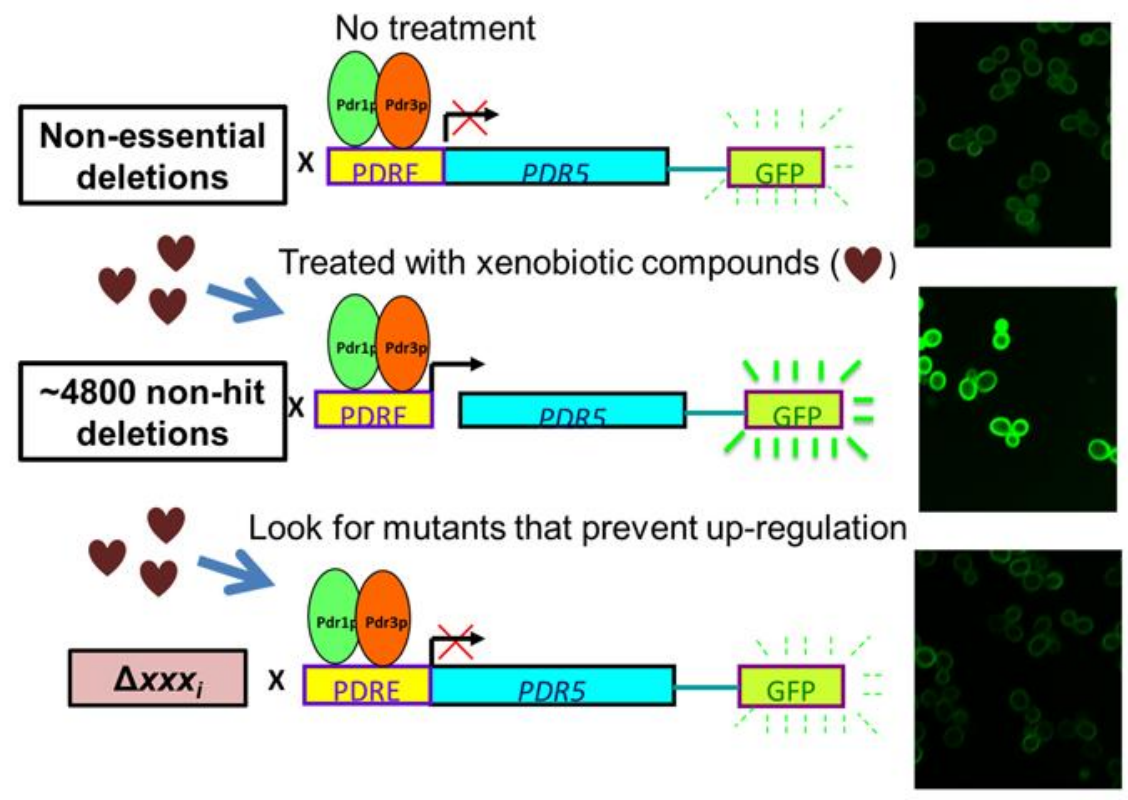

Figure 1-23: Schematic presentation of PDR efflux pumps GFP reporters

\section{Identification of regulators/sensors in different stress responses}

As described above, PDR is a complex network involving interactions between transcriptional activators, also mediator complexes, where we might expect there would be overlap between particular processes and other stress responses, such as oxidative stress response. As previously mentioned Yap1p might link between these two stress responses. A better understanding of the Yap1p regulatory role could help us understand more about these two stress pathways.

Under low-oxidative stress conditions, Yap1p is found in the cytoplasm. Upon oxidative stress induction, Yap1p re-localised to the nucleus, where it activates its target genes (Kuge, Jones et al. 1997). To investigate genes that have roles in regulating Yap1p localization in the presence of different oxidants, we constructed a Yap1p-GFP reporter and repeated the screen with this reporter under oxidative stress induced by hydrogen peroxide $\left(\mathrm{H}_{2} \mathrm{O}_{2}\right)$ and diethyl maleate. In these screens, we looked for genes that prevent nuclear re-localisation upon oxidative stress induction. These genes again are the potential upstream mediators and regulators (Figure 1-24). In the results section, we compared the efflux pump reporter 
screens with the Yap1p reporter screens to see if there are any common regulators or modulators between these two stress response pathways.

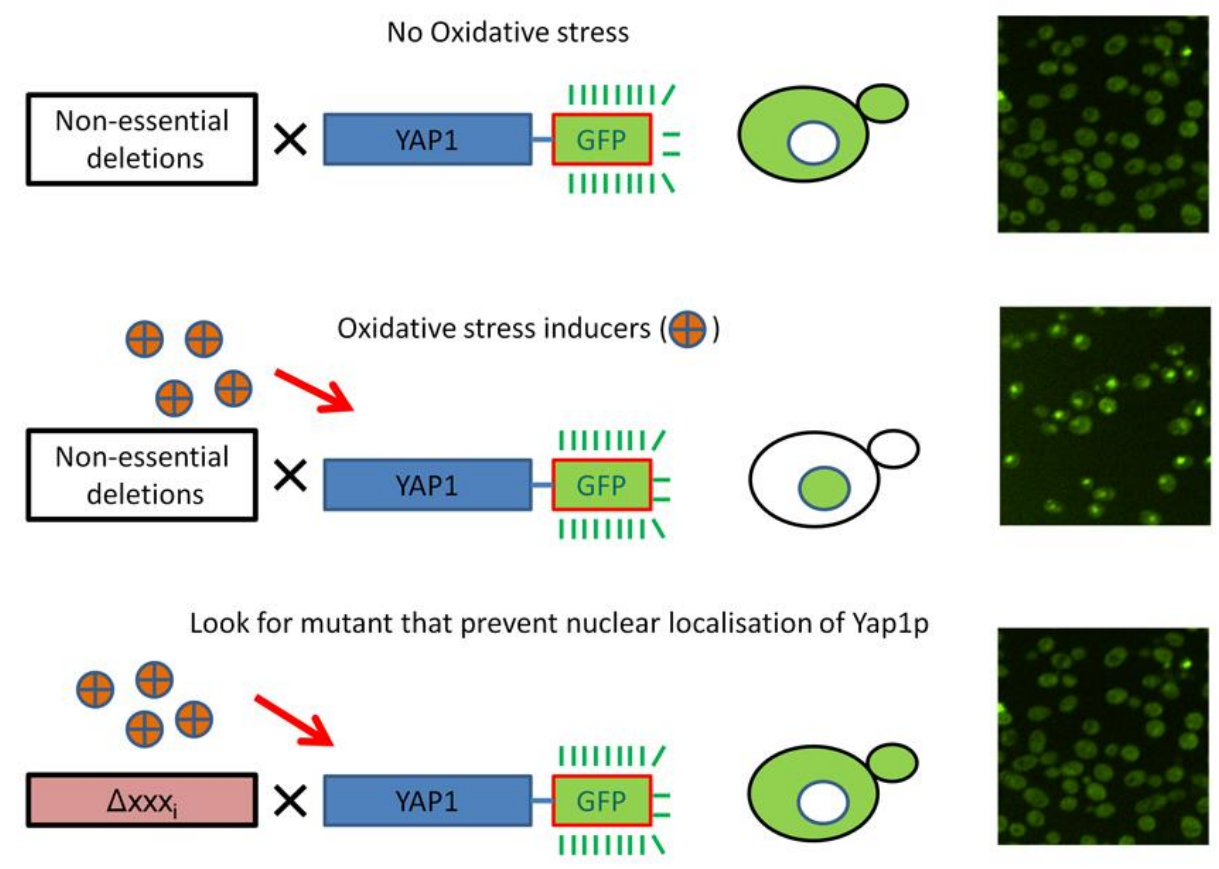

Figure 1-24: Schematic presentation of Yap1p_GFP reporter 


\section{Chapter 2. General Materials and Methods}

\section{Compounds}

Unless otherwise stated, all compounds were purchased from Sigma Aldrich, and dissolved in DMSO.

Neothyonidioside (neo) (Chapter3) was a gift from Dr. Peter Northcote from School of Chemical and Physical Science Victoria University of Wellington. The stock concentration is $20 \mathrm{mM}$ in DMSO.

Compounds used in the spot dilution assay to validate PDR primary hits (Chapter 4) are listed in Table 2-1 below

\section{Table 2-1: Compounds used in spot dilution assay}

\begin{tabular}{|c|c|c|}
\hline Compound & $\begin{array}{l}\text { Concentration used in } \\
\Delta x x x \text { background }\end{array}$ & $\begin{array}{l}\text { Concentration used } \\
\text { in } \Delta x x x \Delta p d r 1 \Delta p d r 3 \\
\text { background }\end{array}$ \\
\hline cycloheximide $(\mathrm{CHX})$ & $0.15 \mu \mathrm{g} / \mathrm{ml}$ & $40 \mathrm{nM}$ \\
\hline ketoconazole & $1 \mu \mathrm{M}$ & $0.5 \mu \mathrm{M}$ \\
\hline fluconazole & $31 \mu \mathrm{M}$ & $12 \mu \mathrm{M}$ \\
\hline oligomycin (in YPGE plate) & $0.35,0.5 \mu \mathrm{g} / \mathrm{ml}$ & $0.35 \mu \mathrm{g} / \mathrm{ml}$ \\
\hline benomyl (SC plate) & $60 \mu \mathrm{M}$ with $25 \mathrm{mM}$ HEPES & Was not used \\
\hline atorvastatin (SC plate) & $35 \mu \mathrm{M}$ & $5 \mu \mathrm{M}$ \\
\hline
\end{tabular}

Hydrogen peroxide, diamide, and diethyl maleate stocks were made up in sterile distilled water. The final concentrations were $1 \mathrm{mM}, 1.5 \mathrm{mM}$, and $2 \mathrm{mM}$ respectively (Chapter 5$)$

\section{Yeast media}

Yeast cells were grown in YPD under non-selective conditions, or in appropriate synthetic complete (SC) media under selective conditions at $30^{\circ} \mathrm{C}$ or at $30^{\circ} \mathrm{C}$ with shaking for liquid culture. 
Yeast Peptone Dextrose (YPD) $1000 \mathrm{ml}$

$\begin{array}{ll}\text { Bacto-Yeast Extract } & 10 \mathrm{~g} \\ \text { Bacto-peptone } & 20 \mathrm{~g} \\ \text { Adenine } & 0.12 \mathrm{~g} \\ 40 \% \text { glucose solution (added after autoclaving) } & 50 \mathrm{ml} \\ \text { Agar } & 20 \mathrm{~g}\end{array}$

Yeast Peptone Glycerol Ethanol (Glycerol and/or ethanol as carbon source)

$\begin{array}{ll}\text { Bacto-Yeast Extract } & 10 \mathrm{~g} \\ \text { Bacto-peptone } & 20 \mathrm{~g} \\ \text { Glycerol } & 30 \mathrm{ml} \\ \text { Ethanol (added after autoclaving) } & 30 \mathrm{ml} \\ \text { Distilled water } & 970 \mathrm{ml} \\ \text { Agar } & 20 \mathrm{~g}\end{array}$

$\underline{\text { SC Media } 1000 \mathrm{ml}}$

Yeast Nitrogen Base without amino acid $\quad 1.7 \mathrm{~g}$

MSG $1 \mathrm{~g}$

$40 \%$ glucose solution (add after autoclaving) $50 \mathrm{ml}$

Amino Acid Mixture to suit $2 \mathrm{~g}$

Agar $20 \mathrm{~g}$

GNA (pre-sporulation media) $1000 \mathrm{ml}$

Bacto-Yeast Extract $\quad 8 \mathrm{~g}$

Bacto-peptone $3 \mathrm{~g}$

$40 \%$ glucose solution (added after autoclaving) $250 \mathrm{ml}$

Agar $20 \mathrm{~g}$ 
Sporulation (Spo) Media 1000ml

Potassium Acetate $\quad 10 \mathrm{~g}$

Bacto-Yeast Extract $1 \mathrm{~g}$

Complete Amino Acid Mixture $2 \mathrm{~g}$

$40 \%$ glucose solution (add after autoclaving) $50 \mathrm{ml}$

Agar $20 \mathrm{~g}$ 


\section{Strains used}

Yeast strains used in this study were derived from S288C and their genotypes are listed in Table 2-2.

\section{Table 2-2: Strains used in this study}

\begin{tabular}{|c|c|c|}
\hline Strain & Genotype & Description \\
\hline BY4741 & 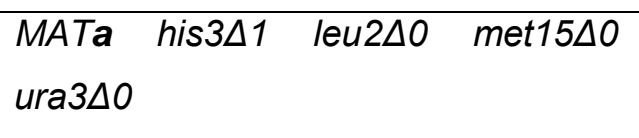 & Wild-type \\
\hline YCG204 & $\begin{array}{l}\text { neo }{ }^{R} \text { MATa can1A:: STE2pr-HIS5 } \\
\text { IypD1:: STE3prLEU2 [CEN-URA3] }\end{array}$ & neo resistant strain \\
\hline YCG253* & 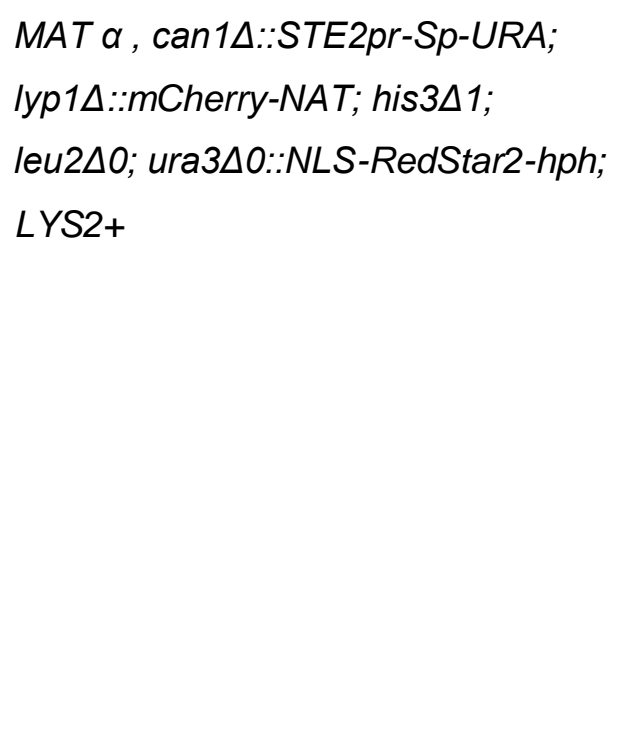 & $\begin{array}{l}\text { SGA starting strain } \\
\text { - mCherry-NAT is red fluorescent protein } \\
\text { (RFP) linked with the NAT(nourseothricin) } \\
\text { dominant selectable marker under a } \\
\text { constitutive promoter It has a cytoplasmic } \\
\text { location. } \\
\text {-NLS-RedStar2-hph is RFP linked with the } \\
\text { hygromycin dominant selectable marker } \\
\text { and with NLS (localised to the nucleus). } \\
\text { Both RFPs act as internal controls and as } \\
\text { markers for automated cell recognition } \\
\text { software. }\end{array}$ \\
\hline YCG255* & 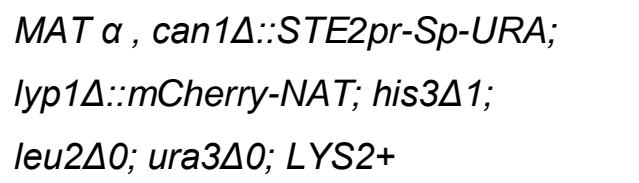 & $\begin{array}{l}\text { SGA starting strain } \\
\text { Please see YCG253 for description }\end{array}$ \\
\hline YCG281 & 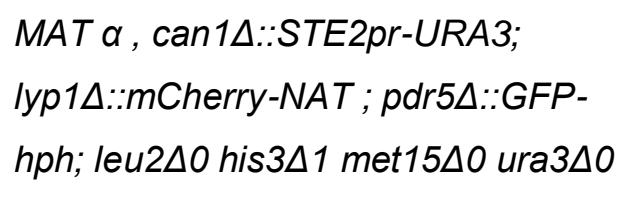 & $\begin{array}{l}\text { Pdr5p reporter strain with PDR5 ORF is } \\
\text { replaced by hygromycin dominant } \\
\text { selectable marker linked with GFP }\end{array}$ \\
\hline YCG285 & 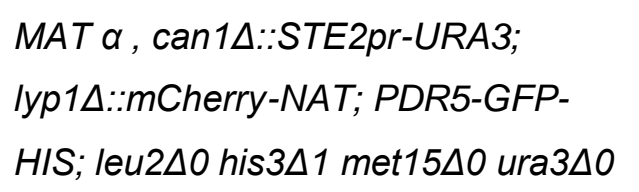 & $\begin{array}{l}\text { Pdr5p reporter strain with C-terminal GFP } \\
\text { linked }\end{array}$ \\
\hline YCG330 & 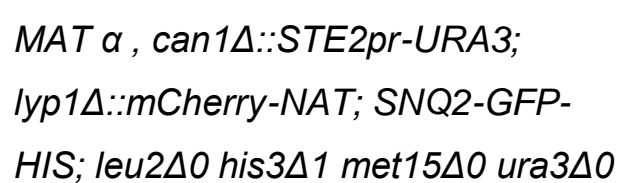 & Snq2p-GFP reporter strain \\
\hline
\end{tabular}


YCG334 MAT $\alpha$, can1 $1:$ :STE2p-URA3; $\quad$ Yor1p-GFP reporter strain

Iyp1A::mCherry-NAT; YOR1-GFP-

HIS; leu2 $\Delta 0$ his $3 \Delta 1$ met $15 \Delta 0$ ura3 $\Delta 0$

YCG379 MAT $\alpha$, can1A::STE2pr_URA3; $\quad$ Yap1p-GFP reporter strain

lyp1 $1:: m$ Cherry-NAT; ura3 $\triangle:$ NLS-

RS2-hph; YAP1-GFP-HIS; leu2 $\triangle 0$

his $3 \Delta 1$ met15 $\Delta 0$

* : these strains were generated by Dr. David Maass, Chemical Genetics Laboratory, School of Biological Sciences, Victoria University of Wellington

\section{Dose response assay}

Cells at a concentration of $5 \times 10^{5} \mathrm{cell} / \mathrm{s} / \mathrm{ml}$ were treated with various concentrations of cycloheximide. DMSO was used as a negative control. After $18 \mathrm{~h}$ incubation at $30{ }^{\circ} \mathrm{C}$, cell density was determined by measuring absorbance at $590 \mathrm{~nm}$ using a spectrophotometer (Perkin-Elmer Wallac Envision 2012). Percent residual growth was calculated using the formula

$$
\frac{A b_{590} \text { treated }}{A b_{590} \text { control }} \times 100
$$

\section{Strain construction}

Yeast strains YCG285, YCG330 and YCG344 were generated by tetrad dissection of spores from crossing YCG255 × Pdr5p-GFP strains, Snq2p-GFP, or Yor1p-GFP respectively. Strain YCG379 was generated by tetrad dissection from crossing YCG253 $\times$ Yap1p-GFP. All the GFP strains were commercial strains from Invitrogen with C-terminal labeled green fluorescent protein (GFP). The haploid

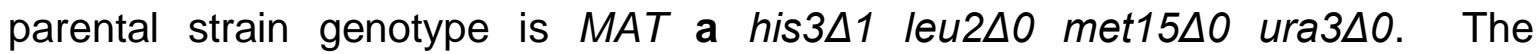
commercial GFP strains were mated against SGA starting strains so that the final progeny strains had the genotype suitable for SGA methodology (see below).

The method for tetrad dissection was as follows:

Fifty microliters of cells grown in liquid sporulation media for 7 days was spun down at $7000 \mathrm{rpm}$ for $15 \mathrm{sec}$, and supernatant was discarded. The cell pellet was re-suspended in $50 \mu \mathrm{l}$ of zymolase in sorbital $(0.5 \mathrm{mg} / \mathrm{ml}$ zymolyse in $1 \mathrm{M}$ sorbitol), and incubated for $10 \mathrm{~min}$ at $30{ }^{\circ} \mathrm{C}$. Three hundred microliters of sterile distilled 
water was added to the suspension and the tube was gently inverted to mix. Fifteen microliters of the suspension was spotted onto YPD plates to separate tetrads. Tetrads were dissected using Singer Dissection Microscope (Figure 2-1).

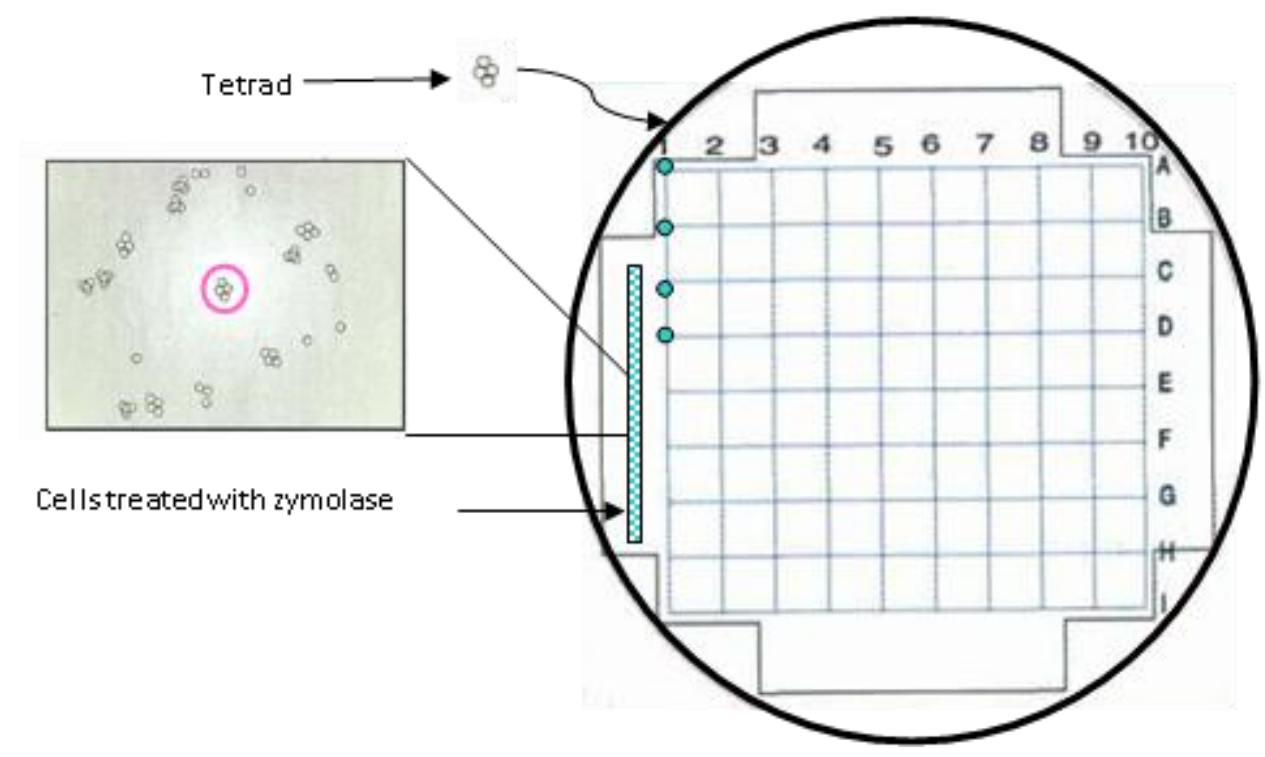

Figure 2-1: Tetrad dissection using Singer Dissection Microscope.

The dissected spores were grown for $2-3$ days at $30{ }^{\circ} \mathrm{C}$, then replica-plated onto selection plates.

For YCG 285, YCG 330 and YCG 344 selection plates were

YPD+ 100 mg/L nourseothricin (clonNAT; Werner BioAgents, Germany) (select for mCherry)

$\left.\begin{array}{l}\text { SC }- \text { Arg }+50 \mathrm{mg} / \mathrm{L} \text { canavanine } \\ \mathrm{SC}-\text { Ura }\end{array}\right\}^{\text {Mating type specific selection }}$

SC - Lys $+50 \mathrm{mg} / \mathrm{L}$ Thialysine (S-Aminoethyl-L-cysteine hydrochloride)

SC- His (selected for GFP)

Spores of the desired genotype listed in Table 2-2 should grow in all conditions except SC - Arg + 50mg/L canavanine (only expressed in Mat a).

For YCG 379 the selection plates were as listed above with extra selection of YPD+ $200 \mathrm{mg} / \mathrm{L}$ of hygromycin (Invitrogen, California). 
Deletion of the PDR5 ORF might increase the difference in GFP intensity changes between control and treated cells. This was tested using PCR and homologous recombination in vivo. The open reading frame of PDR5 was deleted and replaced with the GFP cassette from pYM25 (Janke, Magiera et al. 2004) with a hygromycin selectable marker, while its natural promoter region was kept intact. The forward and reverse primers were

5'AGACCCTTTTAAGTTTTCGTATCCGCTCGTTCGAAAGACTTTAGACAAAAAT GAGTGCTTCTTCTGAAGATGTC3' and

5'TTAAAAAAGTCCATCTTGGTAAGTTTCTTTTCTTAACCAAATTCAAAATTATC GATGAATTCGAGCTCG3' , respectively.

Foot note: The red text represented base pairs complementary to pYM25.

PCR reaction and PCR cycle were as follows:

PCR Reaction of $50 \mu \mathrm{l}$ volume using Qiagen PCR kit

$\begin{array}{ll}\times 10 \text { buffer } & 5 \mu \mathrm{l} \\ \text { Q buffer } & 10 \mu \mathrm{l} \\ \text { Forward Primer } & 0.5 \mu \mathrm{l} \\ \text { Reverse Primer } & 0.5 \mu \mathrm{l} \\ \text { Hot Star Taq } & 0.25 \mu \mathrm{l} \\ \text { dNTP } & 2 \mu \mathrm{l} \\ \text { pYM25 (template) } & 2 \mu \mathrm{l}\end{array}$

Sterile distilled water $29.75 \mu \mathrm{l}$

PCR cycle

$\left.\begin{array}{ll}95{ }^{\circ} \mathrm{C} & 15 \mathrm{~min} \\ 95{ }^{\circ} \mathrm{C} & 1 \mathrm{~min} \\ 55^{\circ} \mathrm{C} & 30 \mathrm{sec} \\ 68^{\circ} \mathrm{C} & 3 \mathrm{~min} \\ 95^{\circ} \mathrm{C} & 1 \mathrm{~min} \\ 55^{\circ} \mathrm{C} & 30 \mathrm{sec} \\ 68^{\circ} \mathrm{C} & 3 \mathrm{~min} \\ 68^{\circ} \mathrm{C} & 10 \mathrm{~min}\end{array}\right\} \times 10$




\section{Screening the library of Pharmacological Active Compounds (LOPAC)}

Reporter strains were grown on SC agar plates for $18 \mathrm{~h}$ at $30^{\circ} \mathrm{C}$ before robotically (Singer RoTorTM) transferred to 384 well PekinElmer Cell Carrier plates (Massachussetts, USA) containing liquid SC. LOPAC compounds (Sigma Aldrich, Auckland, New Zealand) were added to the cell plates using the CyBIO ${ }^{\mathrm{TM}}$ and V\&P Scientific liquid transfer floating pin system. $1 \mu \mathrm{l}$ of compounds were delivered to the recipient plate so that the final compound concentration was $27 \mu \mathrm{M}$. DMSO was used as a control. Cells were incubated for $4 \mathrm{~h}$ at $30^{\circ} \mathrm{C}$ before cell images were taken using automated PekinElmer's Opera $^{R}$ High content microscope (Massachussetts, USA). ). The images were analyzed using Acapella ${ }^{\mathrm{TM}}$ "Texture Analysis" software to automatically identify cells using RFP cytoplasmic mCherry and RFP RedStar2-nuclear localization signal in the analysis of subtle intracellular changes of biological relevance that could be missed by looking at fluorescence intensity alone. The parameters measured by this software were "Spot", “Hole”, "Saddle”, “Edge”, "Ridge”, “Valley”, "Bright", and "Dark” (Figure 2-2).

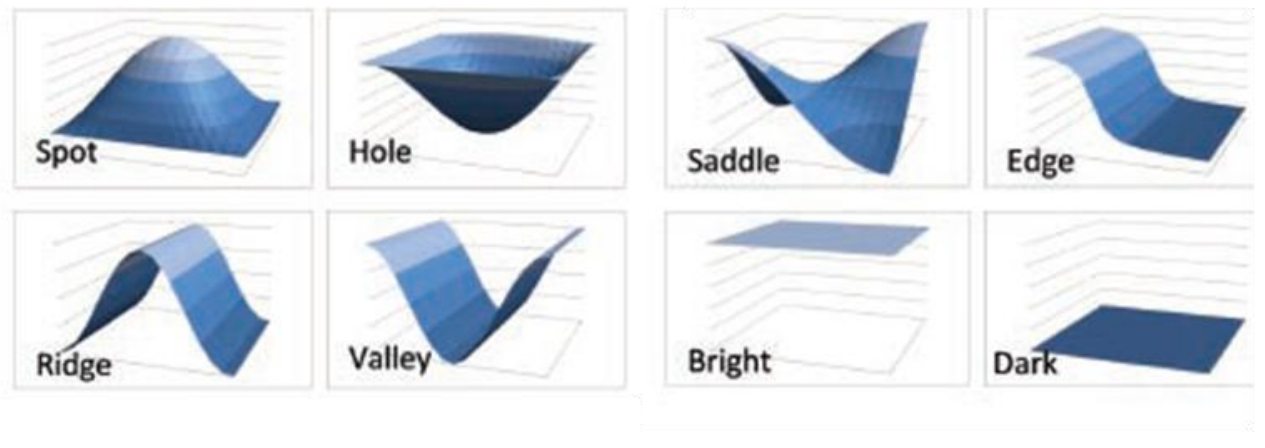

Figure 2-2: Parameter of the Texture Analysis Software

Cellular textures parameter "Ridge", "Bright", and" Edge" were selected due to the fact that they best represented the correct localization of GFP-reporter (Pdr5p, and Yor1p) and gave reproducible results across the different screens.

For each gene deletions, intensity change and change in texture parameters were calculated. For intensity, the fold change between treated and control after normalization against an internal RFP control was used. For texture analysis, Z 
score was calculated according to the equation stated (Materials and Methods $\mathrm{p}$. 71)

\section{Synthetic Genetic Array (SGA) and drug screening}

SGA analysis was carried out according to the method as previously described (Tong, Evangelista et al. 2001)using a Singer RoTorTM HAD bench-top replicator robot (Somerset, UK) in which the Mata query strain was crossed en masse with the MAT a yeast deletion mutant array in 384 colony format. After 2 days incubation at $30{ }^{\circ} \mathrm{C}$ final selections plates were then pinned to SC plates in which plates were arrayed so that each gene deletion was duplicated side by side in 384 format using Singer RoTorTM HAD bench-top replicator robot. Plates were then incubated at $30{ }^{\circ} \mathrm{C}$ overnight, before cells were transferred from colonies to liquid in 384 PekinElmer Cell Carrier plates containing DMSO (control) and drug (atorvastatin $35 \mu \mathrm{M}$ ) in alternate columns. After 4 hours of incubation, cell images were taken using PerkinEImer's OperaR High content microscope. The set up was as follows: 60XWater Immersion lens $N A=1.2$ utilising 488 and 561 lasers with power $(\mu \mathrm{W})$ of 4110 and 731 respectively. Filter for camera 1 (488 laser):520/35, camera 2 (561 laser):600/40, Detect Dichro: 568, Primary Dichro:405/488/561/640. Images were recorded utilising "binning" of 2 and exposure time of $400 \mathrm{msec}$. Images were analysed using Texture analysis in Acapella software with the texture properties of "Ridge", "Bright" and "Edge".

\section{Identification of possible regulators of pleiotropic drug resistance}

In each experiment in the primary screens, $Z$ scores for each deletion were calculated.

$$
\mathrm{Z}=\frac{\left(\left|\bar{X}_{\text {control }}-\bar{X}_{\text {treated }}\right|\right)}{\sqrt{\frac{s_{\text {control }}^{2}}{n_{\text {control }}}+\frac{s_{\text {treated }}^{2}}{n_{\text {treated }}}}}
$$

Where:

$\bar{X}=$ the mean of texture properties (ridge, bright or edge) from texture analysis in Acapella software from each group (control or treated).

$S=$ standard deviation of the texture properties (ridge, bright or edge) ) from texture analysis in Acapella software from each group (control or treated). 
$\mathrm{n}=$ number of cells identified by Acapella software from each group (control or treated).

Gene deletions with the $Z$ score lower than $5^{*}$ in 2 out of 3 texture analysis ("Ridge", "Bright" ,"Edge") were counted as hits. Gene deletions that appeared as hits from 4 out of 5 screens were subjected to further validation.

* 4.753 is the corrected $Z$ value for multiple testing with equivalent significant level of 0.01 . Correction for multiple testing was required because we compared control versus treated for 4500 genes $=4500$ multiple testing. The new corrected $p$-value was $0.01 / 4500=0.000002$.

For the YAP1 screen we also looked at "Spot" property from texture analysis as well as compared intensity of $\frac{\text { NuclearGFP }}{\text { Cytoplasm } G F P}$ between treated and control.

\section{Spot dilution assay}

Yeast cells at a concentration of $5 \times 10^{8}$ cells $/ \mathrm{ml}$ were serially diluted in water (1:10), and were $2 \mu \mathrm{l}$ was spotted on YPD plates (unless stated differently) containing drugs of concentration stated in Table 2-1. Plates were incubated for 2 days at $30{ }^{\circ} \mathrm{C}$ and growth phenotype was observed for each strain.

\section{Yeast cell extract and western blot}

Cell lysate and protein extraction was conducted according to (Egner, Mahe et al. 1995). Cells from different strains were grown in YPD to the same $O D_{600}$ of 2 , and were then harvested by centrifuging at $4000 \mathrm{rpm}$ for $2 \mathrm{~min}$, then washed once with $\mathrm{H}_{2} \mathrm{O}$. Cell extract was prepared by lysising the pellet with $150 \mu \mathrm{l}$ of $1.85 \mathrm{M} \mathrm{NaOH}$ $7.5 \%$ 2-mercaptoethanol for $10 \mathrm{~min}$ on ice, followed by addition of $150 \mu \mathrm{l}$ of $50 \%$ trichloroacetic acid and 10 min incubation on ice. The pellet was collected by centrifugation at $13,000 \mathrm{~g}$ for $3 \mathrm{~min}$ and then re-suspended in $10 \mu \mathrm{l}$ of $1 \mathrm{M}$ Tris base and $50 \mu$ sample buffer $(40 \mathrm{mM}$ Tris- $\mathrm{HCl} \mathrm{pH} \mathrm{6.8,} 8 \mathrm{M}$ urea, $5 \%$ sodium dodecylsulfate (SDS), 0.1M EDTA, 1\% 2-mercaptoethanol, 0.01\% bromphenol blue). Samples were then heated at $42{ }^{\circ} \mathrm{C}$ for $15 \mathrm{~min}$, then $20 \mu \mathrm{l}$ of each samples was loaded to $7.5 \%$ polyacrylamide-SDS gel and electrophoresed for $2 \mathrm{~h}$ at $200 \mathrm{~V}$. The running buffer used was $3.3 \mathrm{~g}$ Tris-base, $14.4 \mathrm{~g}$ Glycine, $1 \mathrm{~g}$ SDS , and distilled water added up to $1000 \mathrm{ml}$. Proteins were then transferred to an 
Immobilon ${ }^{\mathrm{TM}}$ transfer membrane (IPVH00010) pore size $0.45 \mu \mathrm{m}$ which had been washed in methanol for 1-2 min, at $20 \mathrm{~V}$ for $17 \mathrm{~h}$. The transfer buffer used was $3.3 \mathrm{~g}$ Tris-base, $14.4 \mathrm{~g}$ Glycine, $200 \mathrm{ml}$ Methanol, and distilled water added up to $1000 \mathrm{ml}$.

After $17 \mathrm{~h}$, the membrane was blocked with $5 \%$ bovine serum albumin (BSA) in $0.1 \%$ Tween in Tris-buffered saline (TBST) for $1 \mathrm{~h}$ to reduce non-specific binding. Membrane was then washed with TBST for 10 min three times. The membrane was then incubated with a Pdr5p primary antibody (polyclonal Pdr5 (yN-18) antibody raised in goats (Santa Cruz Co., California, USA stock \#SC-27253) diluted 1:500 in 5\% TBST for $3 \mathrm{~h}$ at room temperature. The membrane was then washed with TBST for 10 minutes three times before being incubated with the secondary antibody, Donkey polyclonal antigoat antibody labelled with Cy5 obtained from abcam $\AA$ ab6566 was diluted 1:4000 in 5\% TBST for $1 \mathrm{~h}$ in the dark. The membrane was washed in Tris-buffered saline (TBS) for $10 \mathrm{~min}$ three times before scanning at $635 \mathrm{~nm}$ using Fujiflim FLA-5100 series fluorescent scaner. Pma1p/Pma2p, which are plasma membrane $\mathrm{H}^{+}$-ATPase were used as internal control. Primary antibody for Pma1p/Pma2p raised in rabbits was obtained from Santa Cruz, SC-33735 (diluted at 1:500).Pma1p/Pma2p primary antibody and Cy5 anti-rabbit antibody diluted at 1:4000 were incubated and detected after scanning for Pdr5p. 


\section{Chapter 3. Mechanism of action for a natural product anti-fungal compound, Neothyonidioside, that is not an $A B C$ transporter substrate}

\section{Introduction}

Target identification of bioactive compounds and mechanism-of-action characterization is a major component of drug discovery. Besides the mechanism this knowledge allows an understanding of side effects of a compound as well as enabling design of new compounds with increased efficacy and potency. Bioactive compound and target(s) interactions are usually complex traits genetically, and have historically been quite difficult to unravel using biochemical methods alone.

As mentioned in the general introduction, Chapter 1, genomic methodologies have been proven useful for bioactive target identification and mode-of-action characterization. This chapter describes further genomic approaches including chemical genetic profiling using microarrays, as well as synthetic genetic array mapping (SGAM) to explore drug mode-of-action.

Although $A B C$ transporters pump a broad spectrum of compounds antifungals of the polyene family such as nystatin and amphotericin $B$ are not $A B C$ transporter substrates. Systemic fungal infection presents a major problem in immunocompromised hosts such as cancer and HIV patients (Richardson 2005). Currently, only five classes of antifungal drugs are available in the clinic (Balkis, Leidich et al. 2002; Kanafani and Perfect 2008; Mathew and Nath 2009) which are

i) Azoles, which inhibit biosynthesis of ergosterol, the main sterol in fungal membranes and not present in human membranes.

ii) Polyenes, which binds to membrane sterols and permeabilize the cell, causing leakage of cytoplasmic materials.

iii) Allylamines that block ergosterol biosynthesis by inhibiting squalene epoxidase encoded by ERG1 causing accumulation of squalene which is toxic to the cells.

iv) Echinocandins, which inhibit synthesis of $\beta$ 1, 3-glucan, the major structural polymer of the cell wall.

v) Flucytosine, which inhibits RNA and DNA synthesis. 
The relative dearth of clinical agents coupled with increasing multidrug resistance highlights the need for new antifungals, as well as an improved understanding of antifungal mode-of-action and possible side effects. Natural products have historically provided a wide variety of selective compounds that are highly potent (Gullo, McAlpine et al. 2006). A saponin fraction isolated from the sea cucumber, Australostichopus mollis, is a mixture of triterpene glycoside isomers with a 2.2:1fold excess of neothyonidioside (Avilov, Kalinovskii et al. 1990) and its closely related saponin mollisoside A (Moraes, Norhcote et al. 2004). Unlike neothyonidioside, mollisoside $A$ was shown to have little activity against mammalian cells and is probably an inactive constituent (G.W.Moraes, $\mathrm{PhD}$ thesis 2003) and for brevity we will refer to the mixture as "neothyonidioside" (neo). Neo has potency similar to the polyene antibiotics, nystatin that binds ergosterol, but it has a different mode of toxicity. Unlike nystatin, neothyonidioside does not permeabilize membranes nor does it produce a prodrug (Yibmantasiri, Leahy et al. 2012).

Drug resistant mutants often provide valuable insight into the pharmacodynamics and pharmacokinetics of small molecules. We generated a neo resistant strain using EMS, and applied SGAM methodology to identify the resistance locus. SGAM exploits the fact that mutants in the Yeast Genome Deletion Set (YGDS) are arrayed according to their chromosome position, thus providing a highresolution genetic map. Tightly linked genes exhibit a distinctive pattern of growth inhibition during synthetic genetic array (SGA) analysis (Tong et al. 2001) due to linkage disequilibrium. Theoretically, this growth defect pattern could be used to identify unknown genetic loci. Using SGAM, we identified NCP1, NADPcytochrome P450 reductase, involved in ergosterol biosynthesis as the resistant locus (Yibmantasiri, Leahy et al. 2012).

Although neo binds to ergosterol, the resistant strain has lower cellular ergosterol than wild-type cells. This is unexpected since in most circumstances mutant cells overexpress the compound target(s) to display resistance. We performed chemical-genetic profiling to explore genes involved in this complex trait, in order to understand the interaction between neo and its target and its mode of action, 
Gene Ontology (GO) analysis of neo chemical-genetic profile had shown that genes compensating for neo toxicity are specifically enriched in ESCRT (endosomal sorting) complex genes (Figure 3-1). Nine ESCRT gene deletions, comprising members of all the ESCRT complexes (0, I, II and III), and VPS4, an endosome ATPase providing motive force for ESCRT-III complex action caused neo hypersensitivity. Since multivesicular body (MVB) and autophagic vacuole formation require all the ESCRT complexes, we thus reasoned that endocytosis and multivesicular body (MVB) vesicle formation might be blocked by neo. Neo in this way resembles the polyene antibiotic natamycin that also binds to ergosterol but does not permeabilize the cell membrane rather acting on endocytosis which is known to be dependent on sterol (Munn 2001; Wachtler and Balasubramanian 2006). To test the hypothesis that neo blocked vacuole formation, we used the vacuole-membrane vital dye FM-4-64 FX (Vida and Emr 1995) and observed the phenotype of cells by confocal fluorescence microscopy. We also compared the phenotype of neo treated cells with those that have mutations in vacuolar protein sorting (vps) genes.

There are six morphological classes (A-F) of vacuolar protein sorting (vps) mutants (Raymond, Roberts et al. 1992). Class A mutants show wild-type morphology with a spherical shape of one to three vacuoles as observed in vps35. Fragmented vacuoles were observed in class $\mathrm{B}$, for example vps17. Class $\mathrm{C}$, for example, vps18, shows very small cytoplasmically dispersed fragments of fluorescence. A single enlarge vacuole is characteristic of class $D$ mutant, for example vps15. In Class E mutants, for example vps27, vacuoles are not strongly stained with FM-4-64 dye (Vida and Emr 1995). However, a membrane-enclosed structure observed next to normally single vacuoles was intensely stained in this mutant class. This organelle is thought to be a vacuolar intermediate blocked in this compartment, as well as containing the internalized a-factor receptor (Davis, Horecka et al. 1993; Vida and Emr 1995). A mix of one to three large vacuoles and fragmented vacuoles was observed in vps 1 , class $\mathrm{F}$ mutant. 


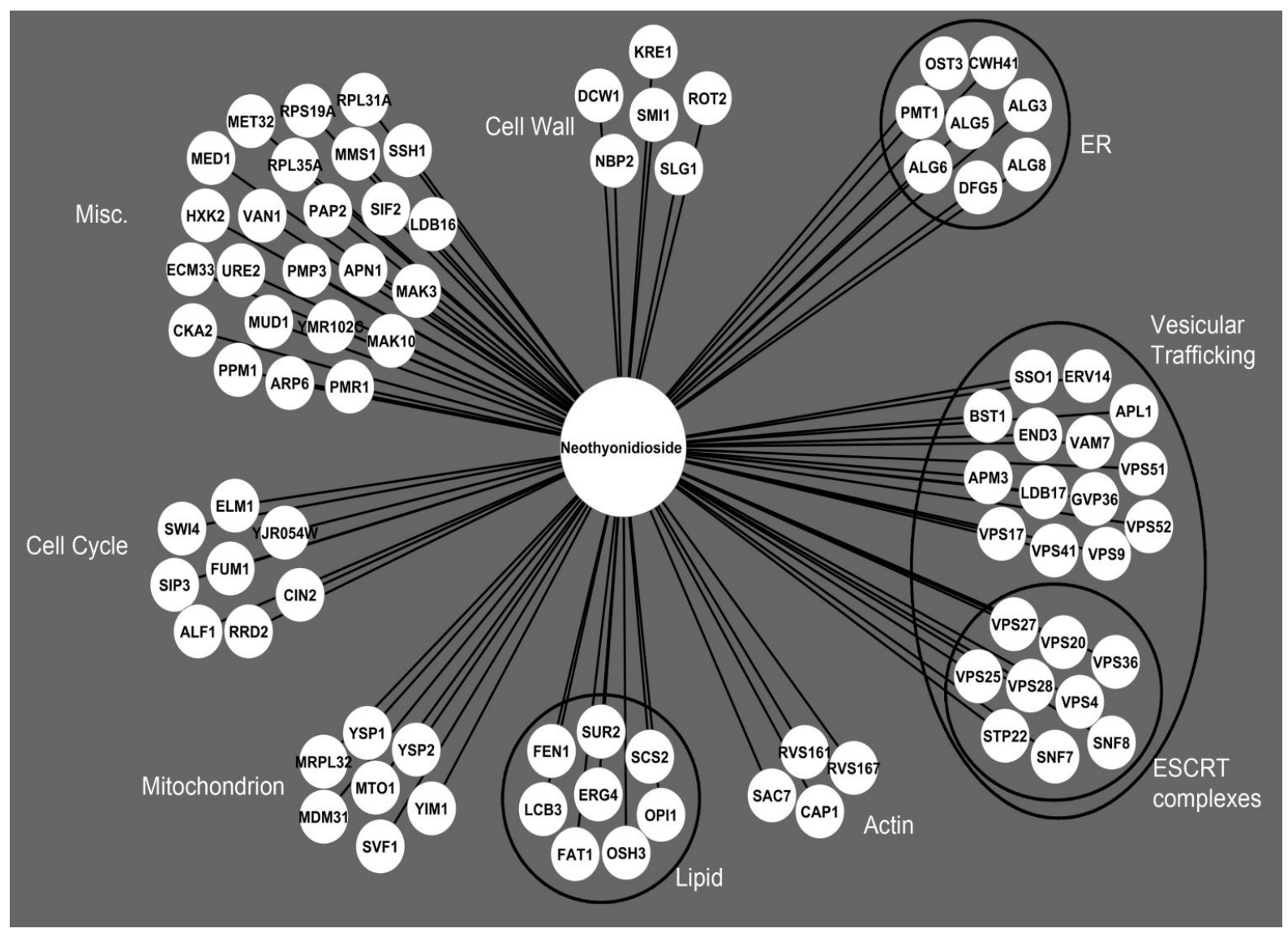

Figure 3-1: Chemical genetic profile of neothyonidioside.

Each node represents a gene deletion that sensitised cells to neothyonidioside

This chapter encompasses a compilation of work published in 2012 (Yibmantasiri et al 2012) focused around endosome-vacuolar intermediates carried out in a number of student projects in this laboratory. The microarray work and chemical genetic profiling (HOP assay) that showed a clustering of ESCRT 0, I, II, III genes buffering the effect of neothyonidioside was performed by S. Andreas Angermayr, Alice G Sorgo and Rose Heathcott. The creation and screening for neo-resistant mutants $\left(n e o^{R}\right)$ was done by Ploi Yibmantasiri in her Honours project utilizing the SGAM technology narrowing down the neo ${ }^{R}$ potential genes to five essential gene candidates. Bede Busby performed the ergosterol quantitation measurements in wild-type and mutant cells. The rest of the mutant characterisation necessary to complete this paper were carried out as part of this dissertation. The microscopy work following the fate of plasma membrane staining dye as a function of the drug neo and as a function of specific deletion mutations was solely part of this dissertation. 


\section{Materials and Methods}

\section{Compound}

Neothyonidioside mixture was provided by Dr. Peter Northcote from the Natural Product Group, School of Chemical and Physical Sciences Victoria University of Wellington. The composition of the compound was determined by Jacqui Barber using high-field NMR spectroscopy and mass specrometry analysis as described in Yibmantasiri et al (2012).

\section{Identification of neo resistant locus using SGAM and chemical-genetic profiling of neo}

For details for the neo resistant strain generation, the identification resistance mediating mutant gene using SGAM, and the chemical-genetic profiling of neo, please see Appendix II.

\section{Vacuole staining and visualisation of live cells}

Vacuole membrane staining with the red-fluorescent dye FM 4-64FX (Invitrogen) was initiated on ice according to Vida and Emr 1995. The strains BY4741 (wildtype), $\Delta v p s 35, \Delta v p s 35, \Delta v p s 17, \Delta v p s 18, \Delta v p s 15, \Delta v p s 27$, and $\Delta v p s 1$ were grown in YPD liquid media at $30^{\circ} \mathrm{C}$ to an $\mathrm{OD}_{600}$ of $0.4-0.8 / \mathrm{ml}$. $1 \mathrm{ml}$ of cells were harvested by centrifugation at $700 \mathrm{~g}$ for $3 \mathrm{~min}$, and resuspended in $50 \mu \mathrm{l}$ YPD. The FM 4$64 \mathrm{FX}$ dye was added to the samples so the final concentration was $40 \mu \mathrm{M}$. In treated cells, a concentration of $0.8 \mu \mathrm{M}(20 \% \mathrm{MIC}$ dose) neo was used. DMSO treated samples were used as controls. Samples were stained for $15 \mathrm{~min}$ at $22^{\circ} \mathrm{C}$ before harvesting by centrifugation at $700 \mathrm{~g}$ for $3 \mathrm{~min}$ and washed in $1 \mathrm{ml}$ YPD followed by $45 \mathrm{~min}$ of "chase" in the absence of dye at $22^{\circ} \mathrm{C}$ in the presence of 0.8 $\mu \mathrm{M}$ neo in the treated cells. Redistribution of the dye after drug addition was observed with an Olympus FluoView FV1000 confocal laser scaning microscope using a $100 x$ oil-immersion objective. 


\section{Results}

\section{Neothyonidioside blocks multivesicular body/vacuole formation.}

In control cultures, labeling of vacuoles was clearly discernible after 45 minutes of dye "chase" (Figure 3-2). Neothyonidioside at $1 \mu \mathrm{M}$ concentration blocked labeling of vacuoles/multivesicular bodies (Figure 3-2). Drug-treated cells showed punctate staining similar to that seen in a $15^{\circ} \mathrm{C}$ temperature block (Vida and Emr 1995), and occasional incomplete vacuole labeling could be observed in some cells in the microscopy fields of our study. Numbers of cells with fully formed vacuoles and the total number of cells observed in the field were counted, and percent of the number of cells with wild-type vacuole morphology, $N_{\text {vacuole }} / N_{\text {total }} \times 100$

was calculated. We found that control samples have nearly three-fold more fully formed vacuoles than neo treated samples (Figure 3-3).

$25 \min$

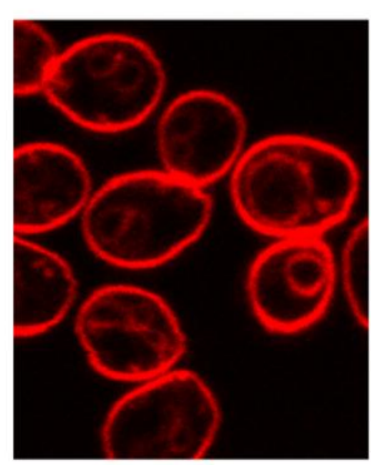

Control

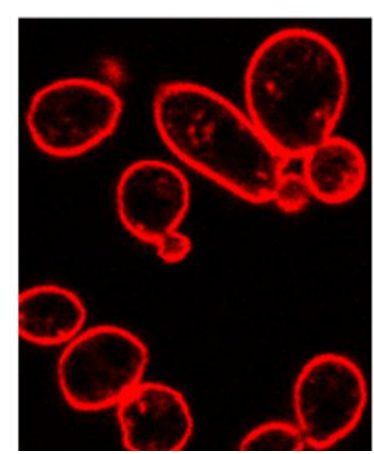

$60 \mathrm{~min}$
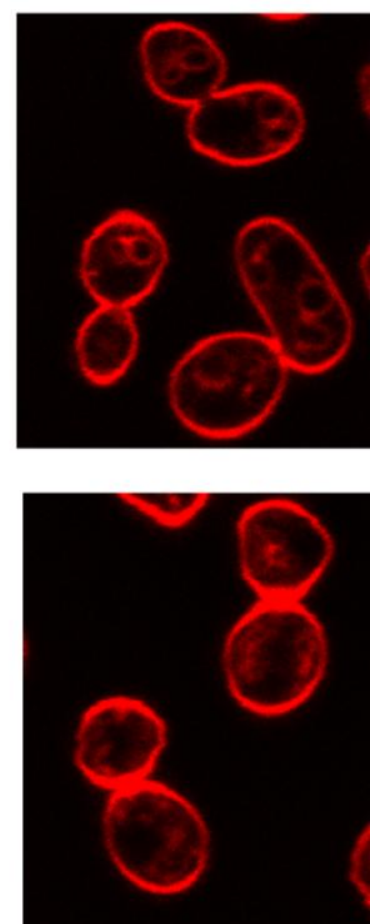

Figure 3-2: Plasma membranes and vacuole membranes in wild-type cells un-treated and treated with neo was stained with FM 4-64 FX

Cells were stained with FM 4-64FX according to Vida and Emr 1995. Cells were visualized using an Olympus FluoView FV1000 confocal laser scanning microscope as described in Materials and Methods for this Chapter. 


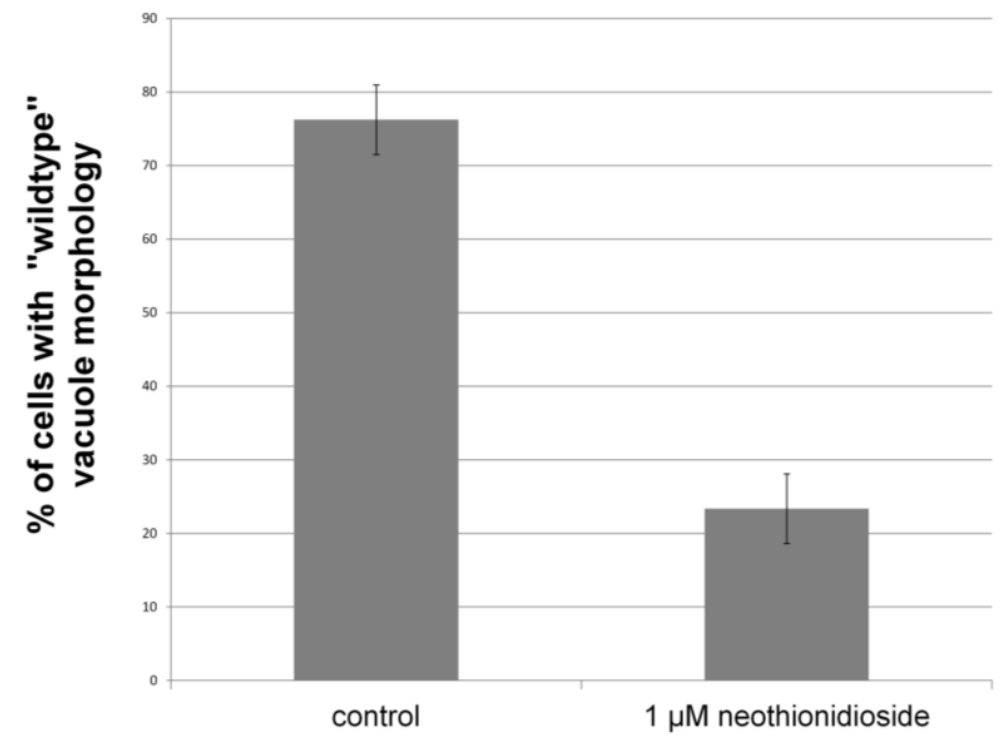

Figure 3-3: Percentage of cells with wild-type vacuole morphology in control samples and treated samples.

Cells from 5 samples of control and drug treatment were counted, $n=471$ and 348 respectively

Neothyonidioside treated cells show similar morphology to class $B$ and $E$ vps Mutants.

Vacuole morphology of wild-type cells, cells treated with neo and vacuolar protein sorting (vps) mutants stained with FM-4-64 FX was investigated as described in the Materials and Methods section of this chapter. Cells treated with a sub-lethal concentration of neo showed defects in vacuole formation similar to those observed in $\Delta v p s 17$ and $\Delta v p s 27$ (Figure 3-4). 

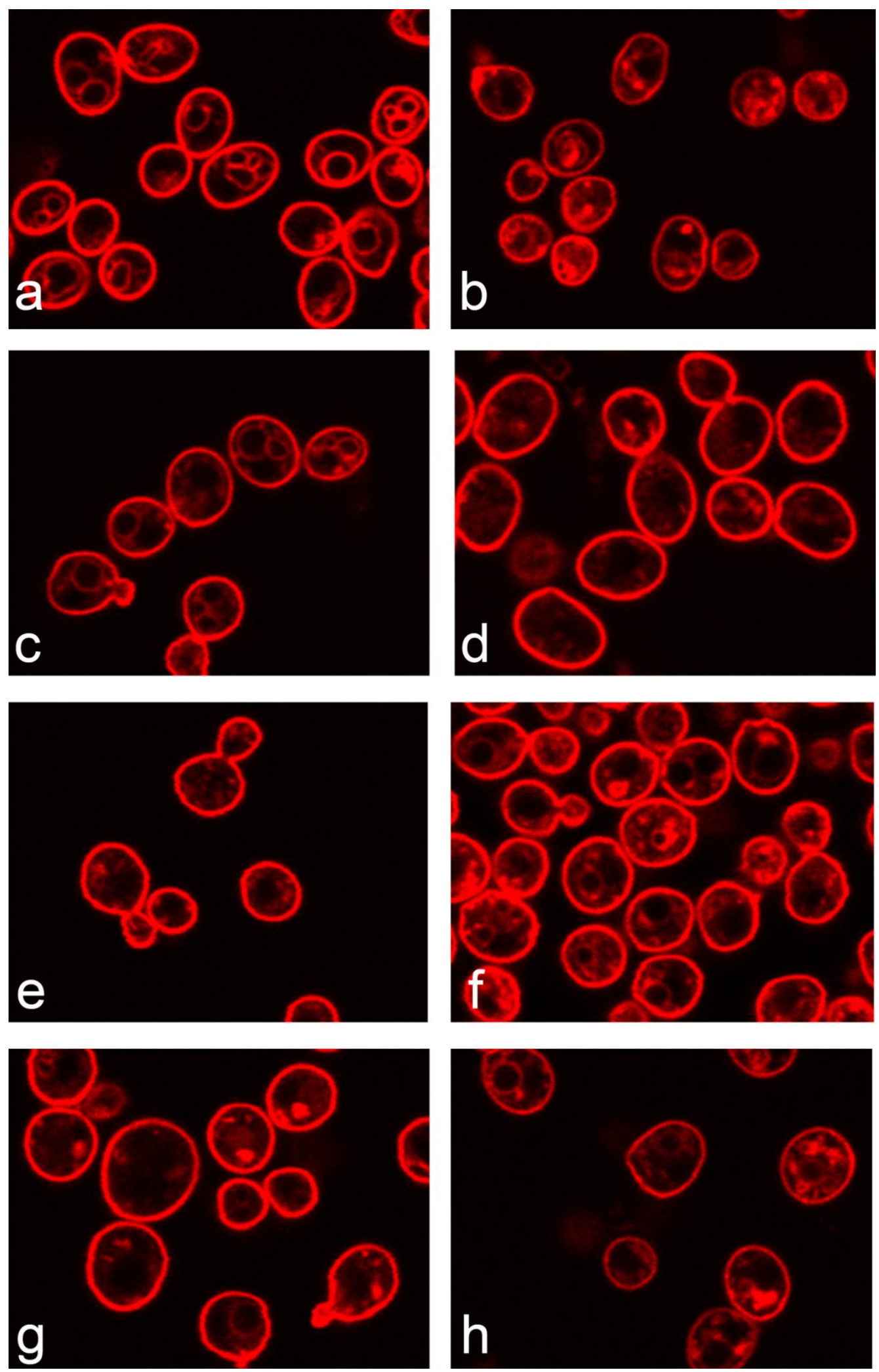

Figure 3-4: Vacuole morphology of neo treated cells and vps mutants.

(a) wild-type control, (b) wild type with sublethal concentration of neo, (c) $\Delta v p s 35$, (d) $\Delta v p s 17$, (e) $\Delta v p s 18$, (f) $\Delta v p s 15$, (g) $\Delta v p s 27$, (h) $\Delta v p s 1$ 


\section{Neothyonidioside resistant mutant showed that wild type vacuole morphology}

Our published study showed that neo resistant cells $\left(n e o^{R}\right)$ have half the amount of cellular ergosterol compared to wild-type (Yibmantasiri, Leahy et al. 2012). To investigate if the neo resistant mutant developed in that study showed any change in vacuole morphology due to changes in ergosterol content we stained the wildtype and mutant cells with FM-4-64FX. The results in Figure 3-5 showed that the mutant cells have the same vacuole morphology as wild-type

\section{wildtype}

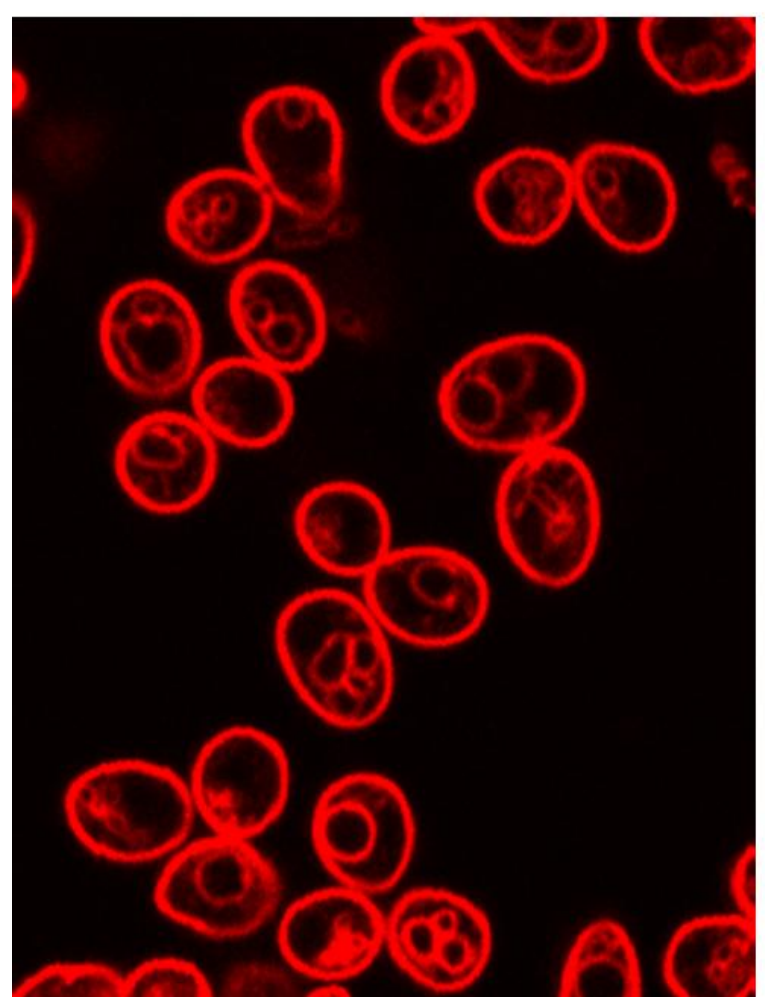

\section{neo ${ }^{R}$}

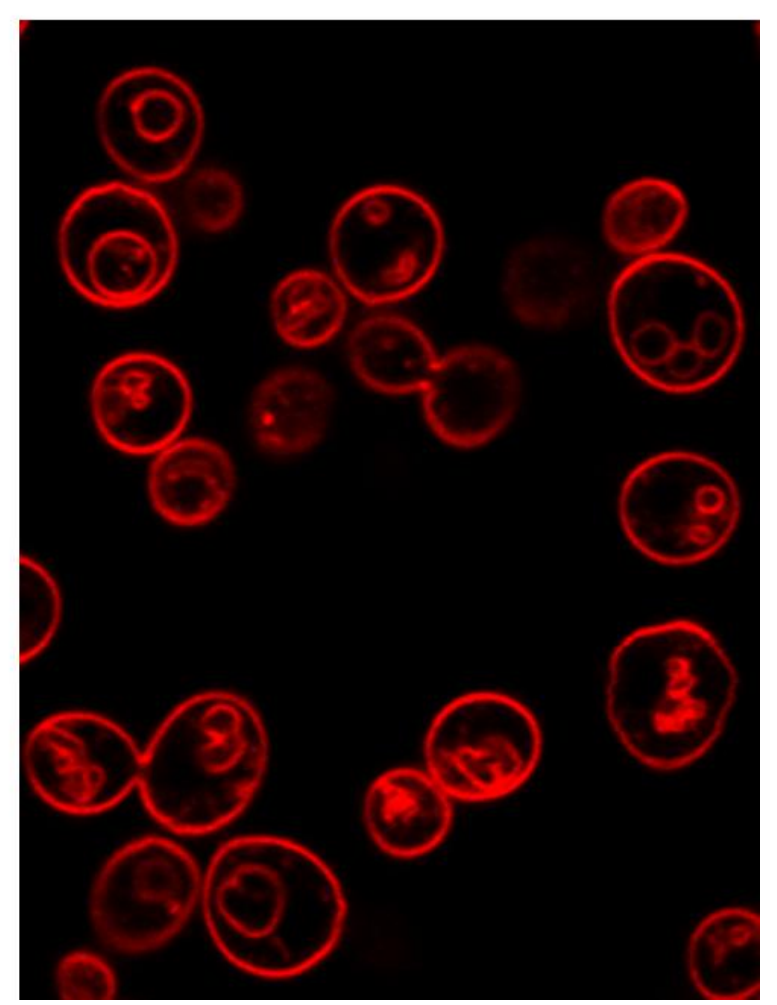

Figure 3-5 : Vacuolar morphology of wild-type cells and neothyonidioside resistance mutant cells (neoR) 


\section{Discussion}

We have shown in our published study that neothyonidioside (neo) is cytotoxic, directly binds to ergosterol, and is not a main ABC transporter substrate (knockout of PDR1, PDR3, PDR5, SNQ2, and YOR1 did not increase cell sensitivity to neo (Yibmantasiri, Leahy et al. 2012). Deletion of genes involved in ergosterol biosynthesis (erg -null mutant) did not cause extra sensitivity to neo. However, a null mutation in ERG3 increased neo tolerance (Figure 3-6; Kristina Boeger summer student report). ERG3 encodes a C-5 sterol desaturase which catalyzes the formation of a C-5(6) double bond into episterol, a precursor in ergosterol biosynthesis reducing the amount ergosterol product (Arthington, Bennett et al. 1991). It has been shown that disruption of ERG3 causes resistance to polyene antifungals such as nystatin and amphotericin (Woods, Bard et al. 1974; Balkis, Leidich et al. 2002) both non-ABC substrates. We found that neo resistant cells have half the amount of cellular ergosterol compared to wild-type cells which is a fundamentally different response to the classic resistance mechanisms in which resistant cells usually increase amount of drug target(s) (Yibmantasiri, Leahy et al. 2012). This suggests to us that neo does not simply bind and sequester ergosterol but has a more complicated mechanism.

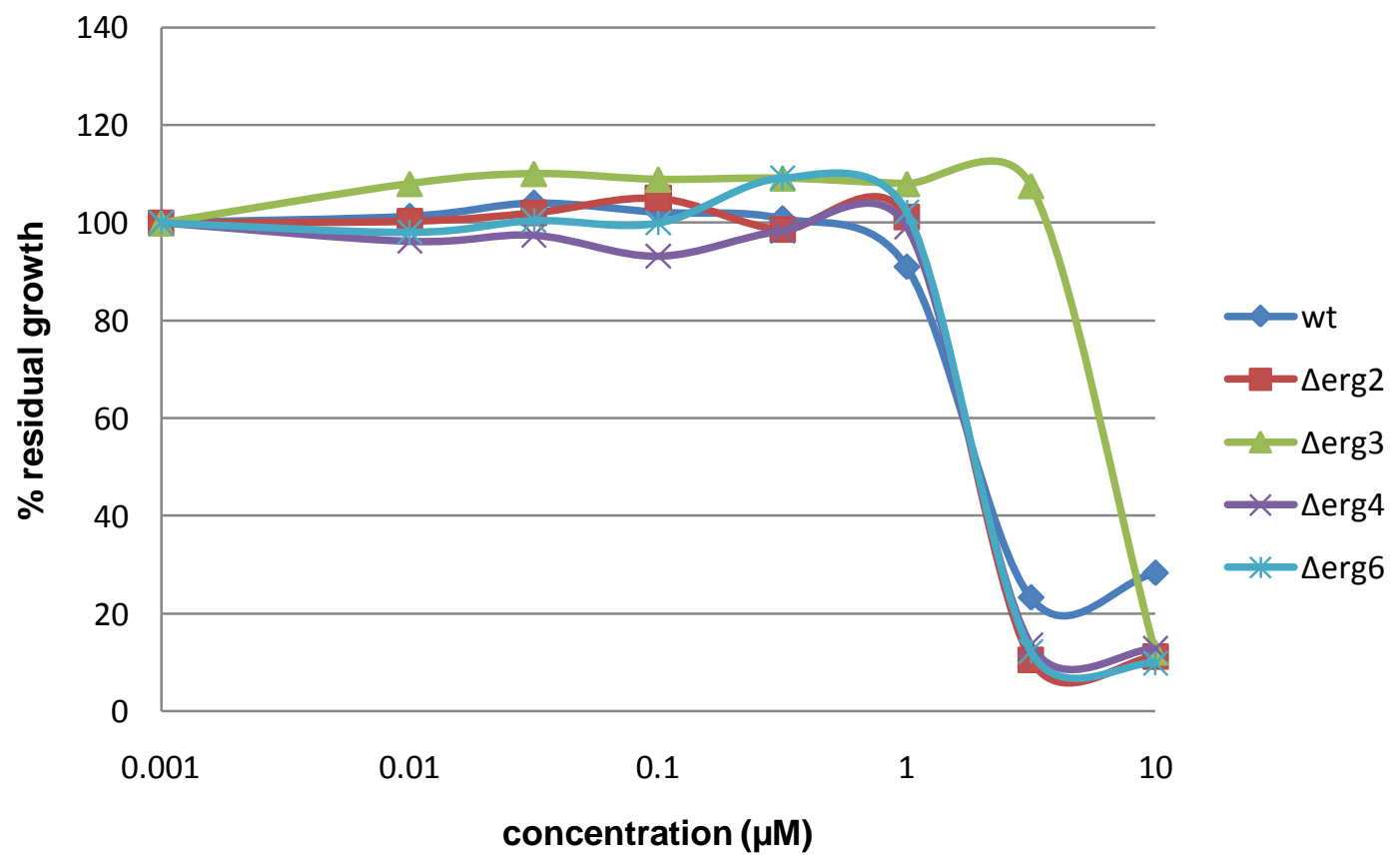

Figure 3-6: Susceptibility of erg mutants to the tolerance to neothyonidioside 
Because of the enrichment of ESCRT genes observed in the chemical-genetic profiling HOP-assays, we hypothesised here that neo might exert its toxic effect via vacuole formation similarly to the antifungal polyene natamycin which binds to ergosterol but does not permeabilize the cell membrane(te Welscher, Napel et al. 2008). This suggested that neo might exert its toxicity by blocking endocytosis and vacuole formation. This prediction was borne out by the microscopy experiments observing the fate of a vital stain for endocytosis of plasma membrane to vacuoles. We observed a blockage in vacuole membrane staining with a build-up of punctuate staining, probably prevacuolar, in the presence of neo (Figure 3-2). In control cultures, labeling of vacuoles was clearly discernible after 1 hour of treatment (Figure 3-2). Neo at this concentration blocked labeling of vacuoles/multivesicular bodies after 1 hour of treatment (Figure 3-2) where only $30 \%$ of the control level of vacuoles was observed (Figure 3-3). Labelling with FM-4$64 \mathrm{FX}$ in neo treated cells was similar in the deletion mutants, $\Delta v p s 17$ and $\Delta v p s 27$ Figure 3-4. Both of these genes were identified as hits in chemical-genetic homologous deletion profiling (HOP) assay. This confirmed our hypothesis that neo disrupts vesicular formation similar to vps mutant and formation of these vesicles is essential in overcoming the drug effect.

An endosome-vacuole pathway alteration is not the only effect of neo because Vps17p is a subunit of the membrane-associated retromer complex and assembles with $\operatorname{Vps} 5 p$ to promote vesicle formation in an endosome Golgi retrograde transport pathway. Vsp27p is an endosomal protein and a member of ESCRT-0 complex, which is required for recycling Golgi proteins. These results strongly support that neo affects both endocytosis process as well as vesicle/ multivesicular body formation involving the ESCRT complex.

Endocytosis of plasma membrane receptors has been shown to involve genes encoding both endocytosis-associating proteins, including those in lipid rafts, clathrin, clathrin-adaptors as well as the ESCRT complex (Burston, MaldonadoBáez et al. 2009). Our results, which showed that neo can affect both endocytosis and multivesicular body formation further support the view that these two processes interact with one another and are essential for cell survival (prevention of these processes by neo results in cell death). 
The neo morphological effects, however, are different from a polyene antifungal, natamycin ,which causes multiple vacuoles formation (te Welscher, Jones et al. 2010), whereas neo appears to block prior to vacuole/MVB formation as shown here (Figure 3-2). We also note that, beside deletion mutants in ESCRT complexes, deletion mutants of the GARP complex (VPS51 and VPS52) and vesicle transport genes (VPS5, VPS41, VPS17), also endocytosis gene deletions (END3, CAP1, RVS167, RVS161) appear in our chemical-genetic profiling as showing hypersensitivity to neo. This is further evidence pointing to the existence of compensating membrane-recycling pathways in response to the neo treatment.

It is possible that by binding to ergosterol, neo, which itself has a sterol-like core, imparts too much rigidity to specific parts of the membrane reducing its ability to bend and form multivesicular body vesicles presumably essential for membrane recycling and lysosomal degradation (Gruenberg and Stenmark 2004; Wollert and Hurley 2010). Increasing sterol concentration is known to increase membrane curvature rigidity and different forms of sterols have different rigidity properties (Gondré-Lewis, Petrache et al. 2006; Henriksen, Rowat et al. 2006). The notion that ergosterol rigidity is involved in neo toxicity is supported by our published work that showed that an ergosterol threshold level is necessary for toxicity. It follows that by reducing ergosterol levels below the required toxicity threshold, the cells become resistant to neo explaining the neo ${ }^{R}$ phenotype. We also observed that $n o^{R}$ cells can still form vesicles/vacuoles the same way as wild-type even though neo $^{R}$ cells have half the amount of cellular ergosterol (Figure 3-5) (Yibmantasiri, Leahy et al. 2012). This suggested that it was the disruption of sterol distribution by neo, and hence prevention of bending of the membrane, and not the amount of sterol on plasma membrane that caused toxicity. ESCRT genes probably compensate neo toxicity by providing aid to membrane inward budding. Taken together, our results suggest a model in which the neo triterpene glycoside binds directly to plasma membrane sterols and at a critical sterol concentration forms a complex that interferes with multivesicular body vesicle formation in endosome intermediate steps.

Although neo is not an $A B C$ substrate, we cannot be sure that neo binding to ergosterol does not trigger a signalling pathway that activates Pdr1p/Pdr3p thereby activating lipid alterations (see Chapter 4). It might be that Pdr1p/Pdr3p is 
activated but neo is not the right substrate to be pumped out by the main $A B C$ pumps. What is clearly shown here however, is a link between lipid composition and drug resistance. Change in lipid composition allows cells to be more or less susceptible to drugs as well as affecting many pathways such as vacuole formation and endocytosis.

It has been known that cells can transport drugs to specific cellular compartment for degradation (Kuchler and Schüller 2007). This compartment-specific drug sequestering involves vacuoles and multivesicular formation, which require ESCRT complex. Genes encoding components of ESCRT complex also came up in chemical -genetic profiling of polyenes antifungal drugs such as amphotericin and nystatin but not as enriched as found with neo (Parsons, Brost et al. 2004; Parsons, Lopez et al. 2006). This result emphasizes the importance of ESCRT complex and vacuoles as ways to compensate for drug toxicity as well as mechanism for multidrug resistance besides the classic up-regulation of $\mathrm{ABC}$ transporters.

Lastly, we have shown that we can determine a drug target and mechanism by genome-wide deletion analysis assuming a drug can act in lieu of a mutation. Although, the actual compound-target binding mechanism required classic biochemistry, chemical-genetics is a fast and powerful method in identifying what pathways the compounds of interest affect, and how cells can compensate for the toxic effects of the compound of interest. 


\section{Chapter 4. Developing the PDR Response Reporters}

\section{Introduction}

Pleiotropic Drug Resistance (PDR) is a robust network achieved by interactions between transcriptional activators, mediator complexes and efflux pumps. The expression of PDR genes is rapidly induced in the presence of xenobiotics through the control of two transcription factors, Pdr1p and Pdr3p. Although the downstream efflux pumps and targets of Pdr1p and Pdr3p are well annotated, the events between sensing of xenobiotics and transcriptional activation by Pdr1p and Pdr3p are poorly understood.

To characterize these events, reporters were developed to investigate genes that are involved in activating/ modulating PDR1/PDR3, hence the multidrug resistance phenotype in yeast. The functional rationale of the reporter(s) was to generate an easily measurable output at specific locations (here is plasma membrane), and dependent on Pdr1p/Pdr3p, as a function of the genome-wide deletion set and the addition of xenobiotic(s).

To achieve this aim, an assay for activation of PDR1 and PDR3 upon treatment with xenobiotic(s) was required. Initially we sought to find change in expression or localisation of the GFP-labeled transcription factors themselves (Pdr1p-GFP and Pdr3p-GFP). However, there were no detectable fluorescent intensity changes upon xenobiotic treatment in the various strains examined. Pdr1p-GFP and Pdr3pGFP also were found localised in the nucleus in both control and treated cells. Therefore, we could not use localisation or fluorescence intensity as a way to measure activation of transcriptional activity of Pdr1p/Pdr3p.

Accordingly, it was decided to use PDR target genes as reporters of Pdr1p/Pdr3p transcriptional activity upon xenobiotic treatment. To this end Pdr5p was chosen because it is the main ABC transporter in S. cerevisiae, being highly expressed in cells with PDR phenotype and dependent on Pdr1p/Pdr3p. It has also been demonstrated to increase expression upon xenobiotic treatment (Balzi and Goffeau 1995; DeRisi, van den Hazel et al. 2000; Gulshan and Moye-Rowley 2007). 
As noted Pdr5p is a naturally high abundance protein in cells, and its use as a reporter requires a detectable increase in fluorescence due to xenobiotic treatment. To cope with possible dynamic range limitations between the basal level of Pdr5p (non-treated cells) and activated level of Pdr5p (addition of xenobiotics) we generated two different reporters.

In the first approach, PDR5-GFP (Invitrogen ${ }^{\mathrm{TM}}$ ), Pdr5p on the plasma membrane was used. The assay was designed to detect increased intensity of Pdr5p-GFP upon activation with xenobiotics detected by Opera ${ }^{\mathrm{TM}}$ confocal microscopy with the analysis software Acapella ${ }^{\mathrm{TM}}$ identifying the plasma membrane region of the cell. This strain (PDR5-GFP) was mated against YCG255 (Table 2-2) the diploid was sporulated and tetrad dissection was conducted as in Materials and Methods (page 67) to yield the hybrid strain YCG285. This provided an appropriate genotype such that the hybrid could be mated against the deletion mutant array and recombinants selected for high-throughput screens in later steps.

In the second approach, the PDR5 open reading frame (ORF) was replaced with GFP, but keeping the promoter intact. This reporter should result in cytoplasmic GFP, where entire cells should turn bright green upon treatment with xenobiotics. It was reasoned that this approach should help to increase the dynamic range between basal level and activation level detected by Acapella ${ }^{\mathrm{TM}}$ software since now the whole cell would be identified instead of only the plasma membrane region. The appropriate PCR product (primers for replacing PDR5-ORF listed in strain construction section in Materials \& Methods from page 67 ) was used to transform YCG255. The transformants were selected in YPD+ $200 \mu \mathrm{g} / \mathrm{ml}$ hygromycin. This generated the reporter YCG281 (Table 2-2 page 66)

In addition to the GFP labels, both YCG285 and YCG281 contained constitutively expressed cytoplasmic red fluorescent protein (RFP) under the LYP1 promoter. RFP was used for automated cell detection by Acapella ${ }^{T M}$ software as well as an internal control. All analyses involving measurement of GFP intensity were normalized against the RFP intensity. To exclude gene deletions which might affect the correct folding and localisation of general proteins (including GFP) and therefore appearing as false-positives, a pre-existing screen from this laboratory (Bircham, Maass et al. 2011) with a plasma membrane protein reporter that is not 
involved in the PDR phenotype was used. Mrh1p is a plasma membrane protein and used in this laboratory to study the unfolded protein response and protein localisation. Gene deletions that were identified as affecting the plasma-membrane reporter levels in both PDR reporter screens and in Mrh1p-GFP screens were regarded as genes required for general plasma membrane folding/localisation and were therefore counted as false positives. These genes were not subjected to further validation.

These reporters allow GFP fluorescence to serve as sensitive real-time indicators for of Pdr1p/Pdr3p activation. Each reporter was mated into the yeast gene deletion background, creating a $\sim 4800$ strain reporter collection as a function of the genome-wide non-essential gene deletions. These new strains were then measured for Pdr5p fluorescence by high throughput confocal microscopy after activation of the PDR response with $35 \mu \mathrm{M}$ atorvastatin, a known PDR-inducer (LIPITOR: Materials \& Methods 71)

In addition to the Pdr5p-GFP reporter on the deletion set background, two other major $A B C$ transporters in $S$. cerevisiae that are Pdr1p/Pdr3p targets namely Snq2p, and Yor1p were used as reporters. Commercial strains from the Invitrogen GFP collection were used to generate SNQ2 and YOR1 reporters similar to the PDR5 reporter, YCG285, already described. The resulting strains for Snq2p and Yor1p are referred to as YCG330 and YCG334 respectively (for genotypes see Materials and Methods Table 2-2 page 66. All three reporters were used for drug screening studies but owing to time limitations, only the Yor1p reporter was mated into the deletion set background as described here for the Pdr5p reporter. 


\section{Results}

To assess whether the ABC-transporters linked to GFP (targets of Pdr1p/Pdr3p) respond measurably to the xenobiotic challenge for use in deletion set screens, we tested YCG285, YCG281, YCG330 and YCG334 in the presence of atorvastatin, a PDR inducer, and the intensity of the GFP reporter of each strain was measured. We found that YCG281 (the strain with PDR5 ORF replaced with the GFP cassette), although the promoter region was intact, it was not used for upregulation upon xenobiotic treatment as anticipated (Figure 4-1). However, YCG285 increased upon atorvastatin treatment by 2.5 fold as measured by Acapella $^{\mathrm{TM}}$ software analysis and therefore this strain was used for further screening.

Analysis of the other two main ABC transporters targets of Pdr1p/Pdr3p, Snq2pGFP (YCG 330) and Yor1p-GFP (YCG334) showed between 1.5-2 fold changes in intensity compared to non-treated cells determined by Acapella ${ }^{\mathrm{TM}}$ software analysis (Figure 4-1).

These results showed that the ABC-transporters labeled with GFP could be used to measure the response to xenobiotic challenge and therefore could be used as the indicator for PDR response activation.

\section{Susceptibility of Efflux Pump Reporters}

To assess that C-terminal GFP tagging does not change the ability of the proteins function normally, a susceptibility assay of the reporters against the PDR substrates cycloheximide (CHX) was conducted. We observed no significant difference in sensitivity between any reporter strain and wild-type (Figure 4-2). 

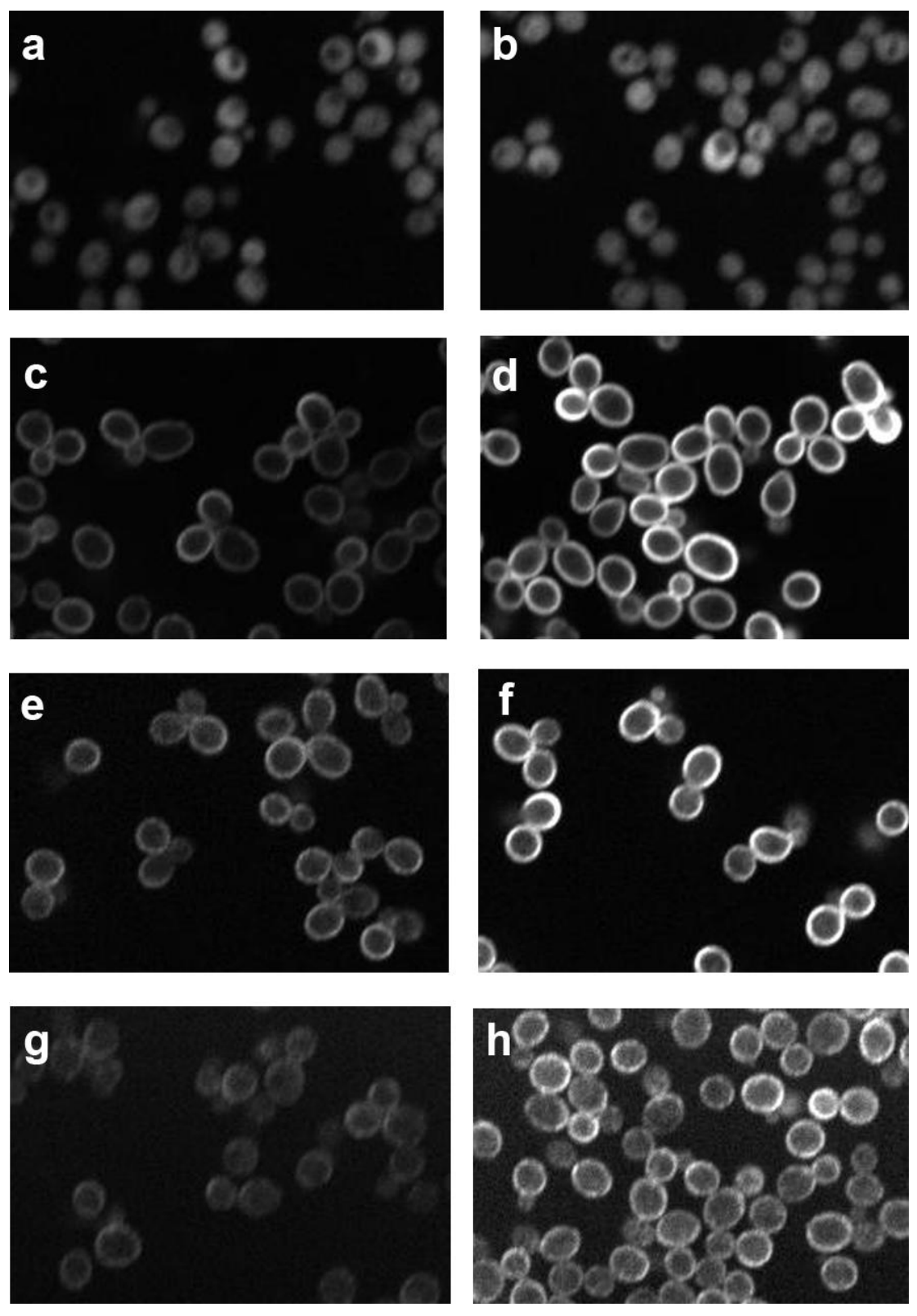

Figure 4-1: ABC transporter GFP reporters.

Left panels: DMSO control. Right panels: $35 \mu \mathrm{M}$ atorvastatin treatment for 4 hours before images were recorded by the OPERA confocal microscope and analysed by Acapella software. a-b) $\triangle p d r 5:: G F P$ (YCG281). c-d) PDR5-GFP (YCG285). e-f) SNQ2-GFP (YCG330). g-h) YOR1-GFP (YCG334). 


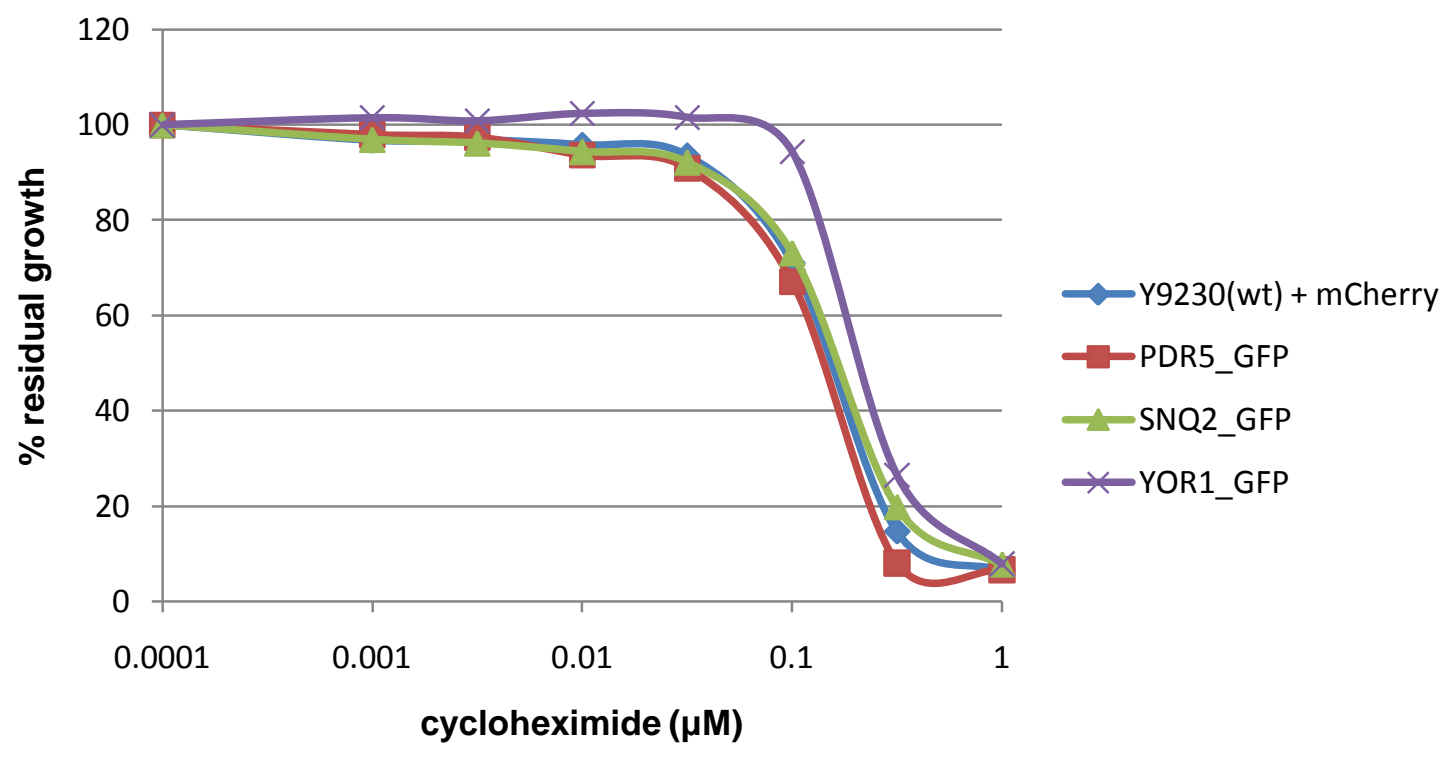

Figure 4-2: Susceptibility of ABC-GFP reporters

Dose response of reporters compared with wild-type (Y9320 + mCherry) at various concentrations of cycloheximide.

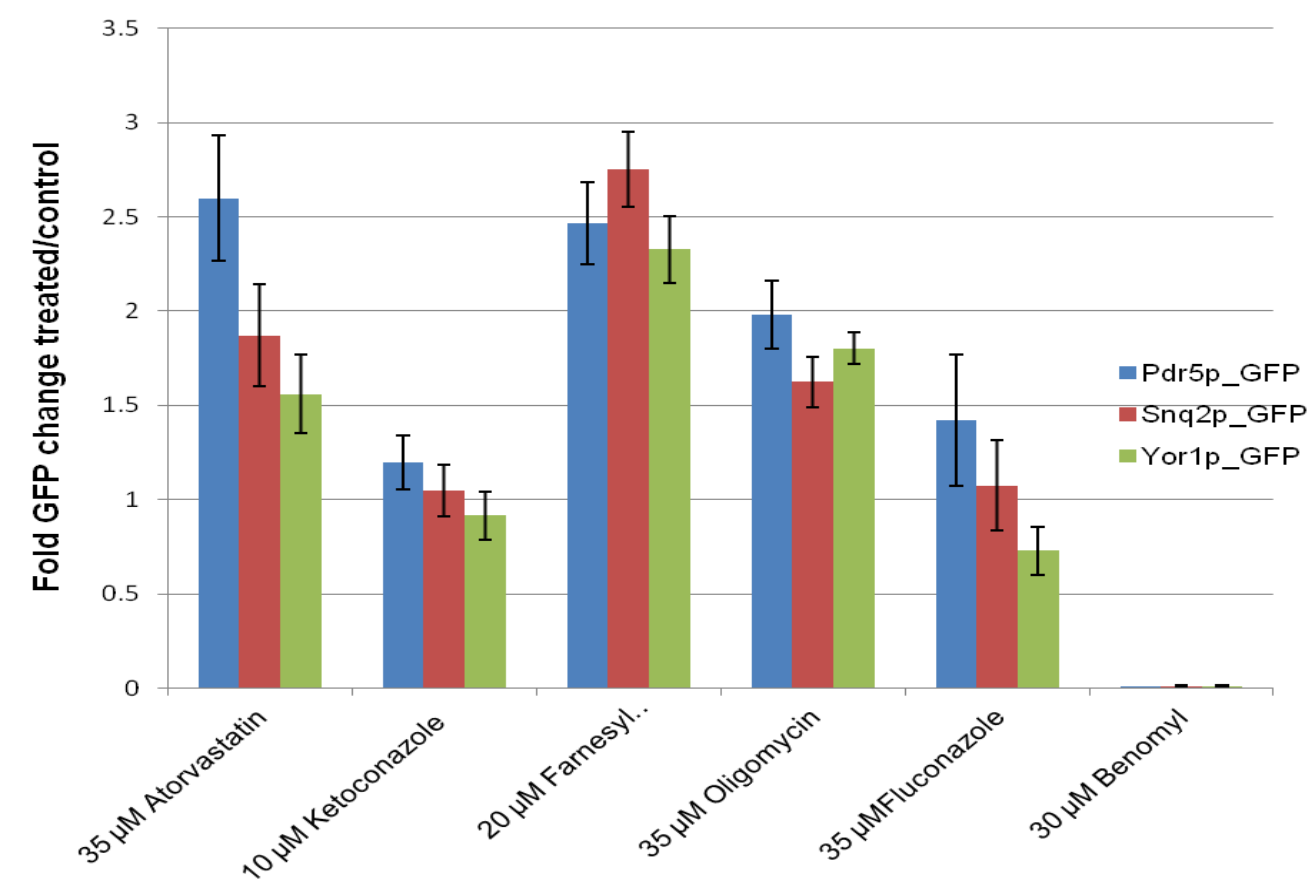

Figure 4-3: Fold change GFP reporters in different xenobiotic treatments (control/treated) Experiments were done in triplicate. 


\section{Selection of Xenobiotics Which Activate PDR Response}

To select xenobiotics that activate the PDR response, a variety of compounds were tested. The compounds used, their concentration, the response to them measuring fold change in GFP between treated/control, in different reporters are shown in Figure 4-3. We found that $35 \mu \mathrm{M}$ atorvastatin, $20 \mu \mathrm{M}$ farnesylthiosalicylic acid (farnesyl), and $35 \mu \mathrm{M}$ oligomycin caused at least a 1.5 fold increased in all GFP reporter expression. With the exception of farnesyl, Pdr5p-GFP reporter expression increased the most among three GFP reporters. Surprisingly, ketoconazole, which is a known Pdr5p substrate, did not cause up-regulation of Pdr5p-GFP.

Reporters were also screened against a library of pharmacologically active compounds (LOPAC ${ }^{1280}$ TM). LOPAC is a library of 1280 high purity, small organic ligands with well-documented pharmacological activities including antibiotics, compounds involved in lipid signalling, gene regulators, phosphorylation etc. All LOPAC compounds were used at a concentration of $27 \mu \mathrm{M}$. To make sure that the used concentration only triggers the PDR response and not other stress responses such as the Unfolded Protein Response (UPR), a reporter strain with a promoter consisting of four tandem repeats of the unfolded protein response element (UPRE) driving GFP expression was screened alongside the PDR reporters. UPR reporter strain was generated by Peter Bircham; SBS, VUW. Only compounds that induced the PDR GFP reporter at least 2-fold over controls and did not up-regulate the UPR reporter were counted as "hits". For example, cerevistatin upregulated the UPR reporter at the concentration used and therefore was not used for future screening. Compounds that induced Pdr5p, Yor1p and Snq2p are listed in Table 4-1.

We found that there was a large collection of compounds that upregulated our PDR reporters. Some upregulated all the $\mathrm{ABC}$-transporter and some were more specific to one or two transporters. We found that more compounds upregulated Pdr5p-GFP reporter than the other two reporters.

Owing to availability and cost efficiency, atorvastatin was used as the compound to activate the PDR response in the primary screen. Atorvastatin has a number of useful attributes including that its target, HMG-CoA reductase, and its associated 
pathway is well known in yeast and higher eukaryotes. Furthermore, the network of the genes sensitive to this drug is available in the lab (chemical-genetic interaction of atorvastatin (Bede Busy personal communication and Master Thesis 2009).

Table 4-1: Compounds from LOPAC Library which upregulated ABC pumps with C-terminal GFP tagged reporters

\begin{tabular}{|c|c|}
\hline & Description \\
\hline \multicolumn{2}{|l|}{ Overlap 3 pumps } \\
\hline Z-L-Phe chloromethyl ketone & Bovine chymotrypsin A-gamma inhibitor \\
\hline 3,4-Dichloroisocoumarin & Serine protease inhibitor \\
\hline Farnesylthiosalicylic acid & Non-toxic Ras inhibitor \\
\hline Phenylbutazone & $\begin{array}{l}\text { Anti-inflammatory; substrate for } \\
\text { prostaglandin peroxidase }\end{array}$ \\
\hline $\begin{array}{l}\text { 3-alpha,21-Dihydroxy-5-alpha- } \\
\text { pregnan-20-one }\end{array}$ & $\begin{array}{l}\text { Positive allosteric modulator of GABA-A } \\
\text { receptors }\end{array}$ \\
\hline Meclofenamic acid sodium & $\begin{array}{l}\text { Cyclooxygenase and 5-Lipoxygenase } \\
\text { inhibitor }\end{array}$ \\
\hline \multicolumn{2}{|l|}{ Overlap Pdr5p and Yor1p } \\
\hline Lonidamine & $\begin{array}{l}\text { Inhibits the energy metabolism of } \\
\text { neoplastic cells by interfering with } \\
\text { mitochondrial hexokinase, cellular } \\
\text { respiration, and glycolysis; damages cell } \\
\text { and mitochondrial membranes }\end{array}$ \\
\hline 5alpha-Pregnan-3alpha-ol-20-one & $\begin{array}{l}\text { Positive allosteric modulator of GABA-A } \\
\text { chloride channels }\end{array}$ \\
\hline L-655,240 & $\begin{array}{l}\text { Thromboxane A2 (TXA2) receptor } \\
\text { antagonist }\end{array}$ \\
\hline \multicolumn{2}{|l|}{ Overlap Pdr5p and Snq2p } \\
\hline Dequalinium analog, C-14 linker & $\begin{array}{l}\text { Protein kinase C-alpha (PKC-alpha) } \\
\text { inhibitor }\end{array}$ \\
\hline Parthenolide & $\begin{array}{l}\text { Inhibits serotonin release from platelets; } \\
\text { inhibits production of leukotriene B4 and } \\
\text { thromboxane B2 }\end{array}$ \\
\hline YC-1 & NO-independent guanylyl cyclase activator \\
\hline
\end{tabular}




\section{Overlap Snq2p and Yor1p}

\begin{tabular}{ll}
\hline Amfonelic acid & $\begin{array}{l}\text { Increases dopamine release during } \\
\text { electrical stimulation; appears to mobilize } \\
\text { dopamine release from storage } \\
\text { compartments }\end{array}$ \\
Cl- channel blocker \\
N-Phenylanthranilic acid & $\begin{array}{l}\text { Blocks LPS- or cytokine-induced activation } \\
\text { of nuclear factor kB (NFkB), which, blocks } \\
\text { the induction of iNOS and COX-2 } \\
\text { transcription; blocks activation of pp70s6k } \\
\text { by all mitogens }\end{array}$ \\
& $\begin{array}{l}\text { Ca2+ ionophenthere used to potentiate } \\
\text { responses to NMDA, but not quisqualate } \\
\text { glutamate receptors }\end{array}$ \\
\end{tabular}

\section{Pdr5p alone}

$\mathrm{N}$-p-Tosyl-L-phenylalanine chloromethyl ketone

Nifedipine

$R(+)$ - Butylindazone

WIN 62577

7-cyclopentyl-5-(4-phenoxy) phenyl7H-pyrolo

DL-stearoylcarnitine chloride

5alpha-pregnan-3alpha-ol-20-one

Diclofenac sodium

Androsterone

Fusidic acid sodium

(Z)-Gugglesterone
Blocks LPS- or cytokine-induced activation of nuclear factor $\mathrm{kB}$ (NFkB), which, blocks the induction of iNOS and COX-2 transcription; blocks activation of pp70s6k by all mitogens

L-type $\mathrm{Ca} 2+$ channel blocker; vasodilator

Potent $[\mathrm{K}+, \mathrm{Cl}-]$-cotransport inhibitor that shows no side effects on the bumetanimide-sensitive [Na+, $\mathrm{K}+, \mathrm{Cl}-]$ cotransport system

NK1 tachykinin receptor antagonist

Potent and selective Ick (src family tyrosine kinase) inhibitor.

Protein kinase $C(P K C)$ inhibitor

Positive allosteric modulator of GABA-A chloride channels

Cyclooxygenase inhibitor; anti-inflammatory

Anabolic steroid

Inhibits protein synthesis in prokaryotes

Bile acid receptor (FRX) antagonist. Has cholesterol lowering activity. 
SU4312

Vascular endothelial growth factor (VEGF) receptor protein tyrosine kinase 1/2 (KDR) and platelet derived growth factor (PDGF) receptor inhibitor

CR2945

Selective gastrin/CCK(B) receptor antagonist

\section{Yor1p alone}

SU 6656

U0126

Bupropion hydrochloride

Actinonin

\section{Snq2p alone}

Bay 11-7085

Nalidixic acid sodium

AIDA

SB-366791

McN-A-343

Salmeterol

\section{Selective Src family kinase inhibitor.}

Specific inhibitor of MEK1 and MEK2 (MAP kinase kinase; MAPKK); also inhibits a constitutively active, mutant form of MEK

Selective dopamine reuptake inhibitor; antidepressant

Leucine aminopeptidase inhibitor
Inhibits cytokine induced IkB (Inhibitor of NFkB) phosphorylation

Inhibits bacterial DNA polymerase (DNA gyrase)

Selective mGluR1 metabotropic glutamate receptor antagonist

Vanilloid receptor-1 (VR1) antagonist

M1 muscarinic acetylcholine receptor agonist.

beta2 Adrenoceptor agonist

\section{Primary Screen Results}

A key question in this section of the dissertation is whether the same set of genes regulates the PDR response when different downstream reporters are used. Two reporters were used namely Pdr5p-GFP and Yor1p-GFP reporters. These two reporters were selected owing to their differences in Pdr1p/Pdr3p dependency. The genotype of these two reporters was adjusted, according to Strains Constructions in Materials and Methods $\mathrm{p}$ 67, for the appropriate selection markers and then each mated against the mutant deletion array to provide the genome-wide deletion background. Because Pdr5p is highly expressed during 
exponential growth and Yor1p is not, both reporter strains were grown for at least 21 hours to make sure that they were in stationary phase and basal expression of both proteins was achieved.

Five screens were conducted; three with the Pdr5p reporter and two with the Yor $1 p$ reporter. In the primary screens, genes of interest are those showing no difference between the mean of the control condition and drug treated condition as identified with Z-scores below 5 . These are the genes that when deleted result in an inability to upregulate PDR5 and YOR1 upon drug treatment, and therefore should be the common modulators of the PDR response. As stated in the introduction of this chapter, genes involved in protein synthesis and protein folding which also came up in Mrh1p control screens were considered as false positives, and were removed from the list. Each screen was analysed independently. Lists of hits from each screen were then compared and gene deletions which came up as hits in at least 4 out of 5 screens were counted toward the final hit list.

From the screens, 54 gene deletions appeared as "hits' (Figure 4-4) i.e. gene deletions that prevented the expected significant increase in Pdr5p and Yor $1 p$ expression upon treatment with atorvastatin. Some imaging methods of were more effective than others in quantifying plasma membrane GFP-reporters. We found that the Acapella "texture analysis" (key components being "ridges", "edges', "bright"; see Materials \& Methods page 71) around the plasma membrane region was the most effective. A "hit" is defined as gene deletions, which showed no significant difference in plasma membrane fluorescence between the control and drug-treated cells. That is to say, such deletions must be affecting PDR (see Figure 4-5). The systematic name, the common name and the description of these "hit" genes are listed in Appendix ITable A-1. 


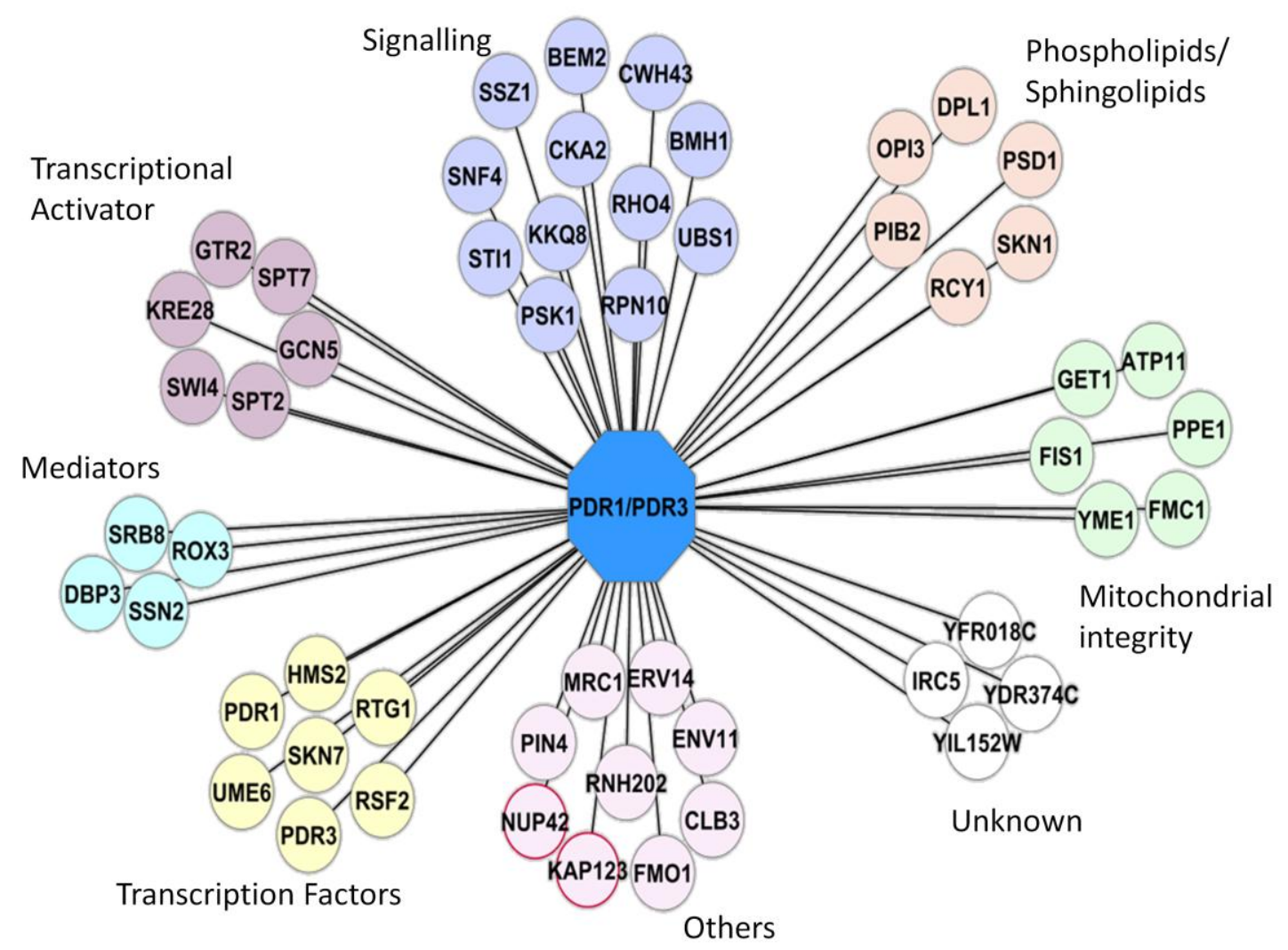

Figure 4-4: PDR Primary screen network.

Gene deletions that prevented normal up-regulation of Pdr5p-GFP and Yor1p-GFP upon atorvastatin treatment.PDR1 /PDR3 are in the middle to represent the known major control of PDR phenotype. Each lines of connection represent the possible regulation relationship between gene $X X X$ and PDR1/PDR3. NUP42 and KAP123 are both nuclear pore complex which have been known to interact with other stress response transcription factors (www.yeastgenome.org)

In analyzing the "hits", Gene Ontology (GO) slim-analysis in the function category showed enrichment of 11 gene deletions in transcription activation and coactivation ( $p$-value 0.05 Table 4-2). Also there was clustering of gene-deletions that are involved in kinase activity ( $P S K 1, S N F 4, K K Q 8$ and $B M H 1)$, genes that are involved in sphingolipid and phospholipid biosynthesis (DPL1, SKN1, PIB2), and genes that are involved in mitochondria integrity (PSD1, FMC1, PPE1, FIS1, $A T P 11$, and YME1), but the transcription cluster was the only one showing GO enrichment with statistical significance.

Transcription factors and mediators relating to RNA-polymerase-II and SAGA complexes associated with regulation of PDR5 were enriched although this is not 
an entirely new finding in the PDR field. Gal11p and Ngg1p have been shown to regulate Pdr1p but enrichment of transcription factors and activators in regulating PDR5 has not yet been described. Such an observation suggests a program of gene transcription is switched on in response to xenobiotics, maybe as part of a stress response.

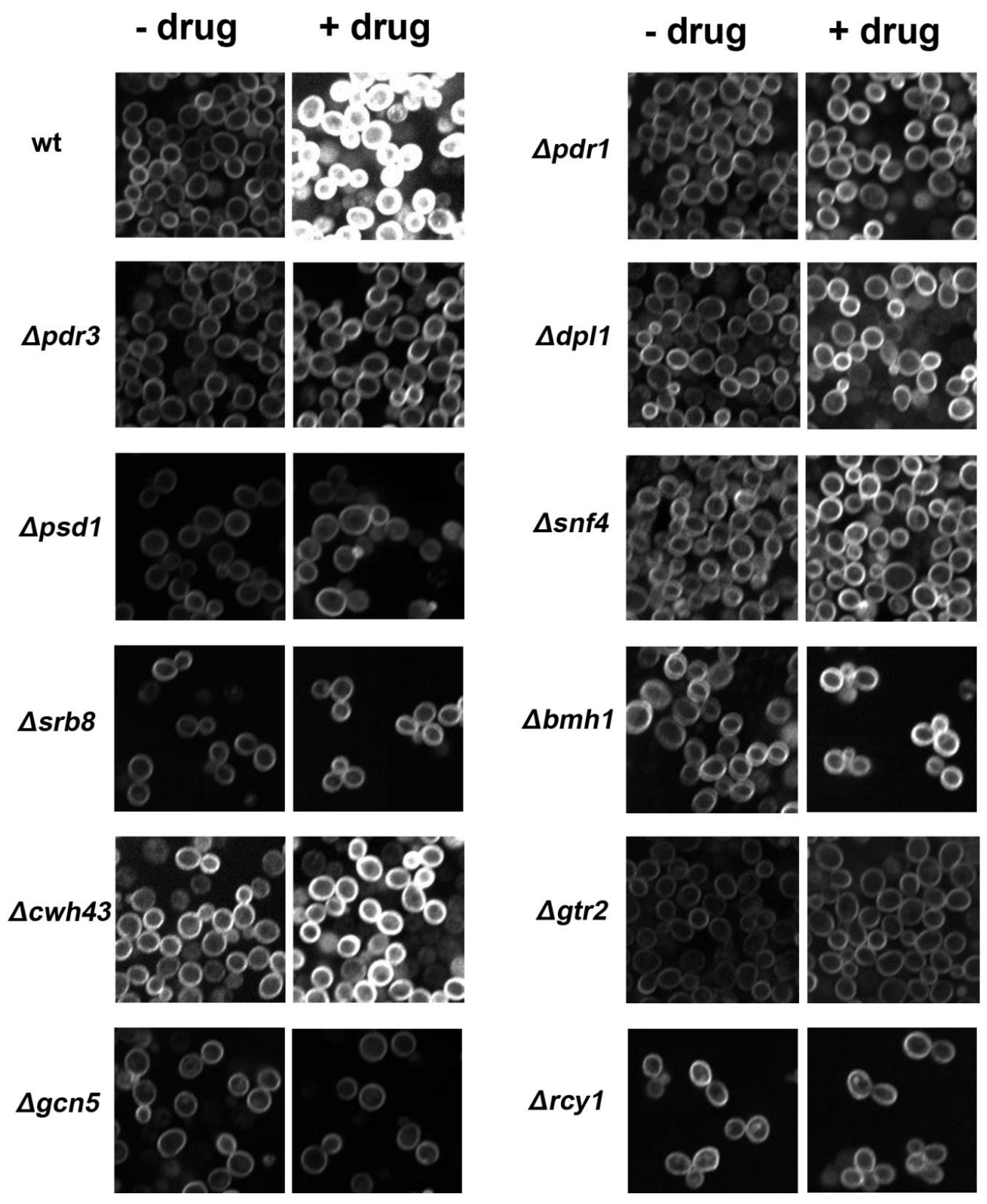

Figure 4-5: Examples of gene deletions that prevented normal upregulation of Pdr5p-GFP and Yor1p-GFP reporters.

The images are of the Pdr5p-GFP reporter in the named gene deletion backgrounds. The images were adjusted so that fluorescence levels of DMSO control and drug treatment of each of the deletions (in $35 \mu \mathrm{M}$ atorvastatin) are directly comparable as to quantity. 


\section{Table 4-2: Eleven Genes relating to RNA transcription were GO enriched as revealed in the PDR primary screens.}

\begin{tabular}{lll}
\hline GO-term & P-value & Gene(s) annotated to the term \\
\hline sequence-specific DNA & 0.00588 & PDR3/YBL005W: \\
binding transcription & & UME6/YDR207C: \\
factor activity & SWI4/YER111C: \\
& & PDR1/YGL013C: \\
& & SKN7/YHR206W: \\
& RSF2/YJR127C: \\
& & RTG1/YOL067C \\
nucleic acid binding & 0.00613 & PDR3/YBL005W \\
transcription factor & & UME6/YDR207C: \\
activity & & SWI4/YER111C: \\
& & PDR1/YGL013C: \\
& & SKN7/YHR206W: \\
& & RSF2/YJR127C: \\
transcription factor & & RTG1/YOL067C \\
binding transcription & 0.04211 & ROX3/YBL093C: \\
factor activity & & SRB8/YCR081W: \\
& & UME6/YDR207C: \\
transcription coactivator & 0.04829 & SSN2/YDR443C: \\
activity & & GCN5/YGR252W \\
& & SRB/YCR081W: \\
\hline
\end{tabular}

\section{Validation of the Primary Screen Hits}

All 54 gene deletion from the primary screens were subjected to further validation using spot dilution assays tested against six known PDR substrates (oligomycin, cycloheximide, fluconazole, ketoconazole, atorvastatin, benomyl). If these genes were important to the cell's survival because of a role in the PDR response it would be expected that their deletion would show increased PDR-drug sensitivity in all cases. At the concentrations used, none of the drugs were lethal to wild-type cells. Strains with double deletion of PDR1/PDR3 were used as a positive control, and showed sensitivity to all the compounds. Gene deletions which showed increased sensitivity or resistance towards compound(s) compared to wild-type were subjected to further studies and were listed in Table 4-3. All experiments were repeated 3 times. We found that 17 gene deletions out of 54 showed an increase in sensitivity toward one or more compounds, 8 of these were sensitive to 
atorvastatin. Interestingly deletion of PDR1 only caused sensitivity toward atorvastatin but none of the other compounds. Examples of genes conferring sensitivity in spot dilutions are shown in Figure 4-6.

\begin{tabular}{|c|c|c|c|c|}
\hline $\begin{array}{l}\text { Gene } \\
\text { (ORF) }\end{array}$ & $\begin{array}{l}\text { Sensitivity in } \\
\Delta x x x\end{array}$ & $\begin{array}{l}\text { Sensitivity in } \\
\Delta x x \times \Delta p d r 1 \Delta p d r 3\end{array}$ & $\begin{array}{l}\text { Reduction } \\
\text { of Pdr5p } \\
\text { in } \\
\text { Western } \\
\text { Blot }\end{array}$ & Description \\
\hline $\begin{array}{l}\text { BEM2 } \\
\text { (YER155C) }\end{array}$ & $\begin{array}{l}\text { Atorvastaytin } \\
\text { Benomyl } \\
\text { Cycloheximide } \\
\text { Ketoconazole }\end{array}$ & $\begin{array}{l}\text { Atorvastaytin } \\
\text { Cycloheximide } \\
\text { Fluconazole } \\
\text { Ketoconazole } \\
\text { Oligomycin }\end{array}$ & - & $\begin{array}{l}\text { Rho GTPase activating } \\
\text { protein (RhoGAP) }\end{array}$ \\
\hline $\begin{array}{l}\text { BMH1 } \\
\text { (YER177W) }\end{array}$ & - & $\begin{array}{l}\text { Fluconazole } \\
\text { Ketoconazole }\end{array}$ & Yes & $\begin{array}{l}\text { 14-3-3 protein regulation of } \\
\text { exocytosis, vesicle } \\
\text { transport, Ras/MAPK } \\
\text { signalling, signalling }\end{array}$ \\
\hline $\begin{array}{l}\text { CWH43 } \\
\text { (YCR017C) }\end{array}$ & - & Oligomycin & - & $\begin{array}{l}\text { Putative sensor/transporter } \\
\text { protein involved in cell wall } \\
\text { biogenesis; }\end{array}$ \\
\hline $\begin{array}{l}\text { DPL1 } \\
\text { (YDR294C) }\end{array}$ & Cycloheximide & - & - & $\begin{array}{l}\text { Dihydrosphingosine } \\
\text { phosphate lyase, regulates } \\
\text { intracellular levels of } \\
\text { sphingolipid }\end{array}$ \\
\hline $\begin{array}{l}\text { FMC1 } \\
\text { (YIL098C) }\end{array}$ & $\begin{array}{l}\text { Cycloheximide } \\
\text { Oligomycin }\end{array}$ & $\begin{array}{l}\text { (Atorvastatin?) } \\
\text { Fluconazole } \\
\text { Ketoconazole } \\
\text { Oligomycin }\end{array}$ & - & $\begin{array}{l}\text { Required for assembly or } \\
\text { stability at high temperature } \\
\text { of the F1 sector of } \\
\text { mitochondrial F1F0 ATP } \\
\text { synthase }\end{array}$ \\
\hline $\begin{array}{l}\text { GCN5 } \\
\text { (YGR252W) }\end{array}$ & - & $\begin{array}{l}\text { Atorvastatin } \\
\text { Cycloheximide } \\
\text { Fluconazole } \\
\text { Ketoconazole } \\
\text { Oligomycin }\end{array}$ & - & $\begin{array}{l}\text { Catalytic subunit of the } \\
\text { ADA and SAGA histone } \\
\text { acetyltransferase } \\
\text { complexes }\end{array}$ \\
\hline $\begin{array}{l}\text { GTR2 } \\
\text { (YGR163W) }\end{array}$ & - & $\begin{array}{l}\text { Fluconazole } \\
\text { Ketoconazole }\end{array}$ & Yes & $\begin{array}{l}\text { Putative GTP binding } \\
\text { protein that negatively } \\
\text { regulates Ran/Tc4 GTPase } \\
\text { cycle; activates transcription }\end{array}$ \\
\hline $\begin{array}{l}\text { KAP123 } \\
\text { (YER110C) }\end{array}$ & - & $\begin{array}{l}\text { Negative genetic } \\
\text { interaction }\end{array}$ & - & $\begin{array}{l}\text { Karyopherin beta, mediates } \\
\text { nuclear import of ribosomal } \\
\text { proteins prior to assembly } \\
\text { into ribosomes and import }\end{array}$ \\
\hline
\end{tabular}




\begin{tabular}{|c|c|c|c|}
\hline $\begin{array}{l}\text { MDM39 } \\
\text { (GET1) } \\
\text { (YGL020C) }\end{array}$ & - & $\begin{array}{l}\text { Fluconazole } \\
\text { Ketoconazole }\end{array}$ & - \\
\hline $\begin{array}{l}\text { OPI3 } \\
\text { (YJR073C) }\end{array}$ & - & Oligomycin & - \\
\hline $\begin{array}{l}\text { PDR1 } \\
\text { (YGL013C) }\end{array}$ & Atorvastatin & $\begin{array}{l}\mathrm{N} / \mathrm{A} \\
\text { (Negative genetic } \\
\text { interaction) }\end{array}$ & Yes \\
\hline $\begin{array}{l}\text { PIB2 } \\
\text { (YGL023C) }\end{array}$ & $\begin{array}{l}\text { Cycloheximide } \\
\text { Ketoconazole }\end{array}$ & $\begin{array}{l}\text { Fluconazole } \\
\text { Ketoconazole }\end{array}$ & Yes \\
\hline $\begin{array}{l}\text { PIN4 } \\
\text { (YBL051C) }\end{array}$ & $\begin{array}{l}\text { Atorvastatin } \\
\text { Benomyl } \\
\text { Cycloheximide }\end{array}$ & - & - \\
\hline $\begin{array}{l}\text { PSD1 } \\
\text { (YNL169C) }\end{array}$ & $\begin{array}{l}\text { Benomyl } \\
\text { Ketoconazole }\end{array}$ & - & - \\
\hline
\end{tabular}

\begin{tabular}{|c|c|c|}
\hline $\begin{array}{l}\text { RCY1 } \\
\text { (YJL204C) }\end{array}$ & $\begin{array}{l}\text { Atorvastatin } \\
\text { Ketoconazole }\end{array}$ & $\begin{array}{l}\text { Negative genetic } \\
\text { interaction }\end{array}$ \\
\hline $\begin{array}{l}\mathrm{RHO4} \\
(\mathrm{YKR055W})\end{array}$ & $\begin{array}{l}\text { Benomyl } \\
\text { Cycloheximide } \\
\text { Fluconazole } \\
\text { Ketoconazole } \\
\text { Oligomycin }\end{array}$ & - \\
\hline $\begin{array}{l}\text { ROX3 } \\
\text { (YBL093C) }\end{array}$ & $\begin{array}{l}\text { Atorvastatin } \\
\text { Cycloheximide } \\
\text { Fluconazole } \\
\text { Ketoconazole } \\
\text { Oligomycin }\end{array}$ & $\begin{array}{l}\text { Negative genetic } \\
\text { interaction }\end{array}$ \\
\hline $\begin{array}{l}\text { RPN10 } \\
\text { (YHR200W) }\end{array}$ & $\begin{array}{l}\text { Cycloheximide } \\
\text { Ketoconazole }\end{array}$ & $\begin{array}{l}\text { Cycloheximide } \\
\text { (Oligomycin?) }\end{array}$ \\
\hline
\end{tabular}

SNF4

(YGL155W) Oligomycin of histones $\mathrm{H} 3$ and $\mathrm{H} 4$

Subunit of the GET complex; involved in insertion of proteins into the ER membrane

Phospholipid methyltransferase catalyzes the last two steps in phosphatidylcholine biosynthesis

Master regulator involved in recruiting other zinc cluster proteins to pleiotropic drug response elements (PDREs)

Protein binding phosphatidylinositol 3phosphate

G2/M phase progression contains an RNA recognition motif, NLS
Mitochondrial inner membrane, converts phosphatidylserine to phosphatidylethanolamine

F-box protein involved in recycling plasma membrane proteins

Small GTPase of the Rho/Rac subfamily of Raslike proteins

Subunit of the RNA polymerase II mediator complex

Non-ATPase base subunit of the 19S regulatory particle (RP) of the $26 \mathrm{~S}$ proteasome

Activating gamma subunit of the AMP-activated Snf1p 


\begin{tabular}{|c|c|c|c|c|}
\hline $\begin{array}{l}\text { SPT7 } \\
\text { (YBR081C) }\end{array}$ & $\begin{array}{l}\text { Cycloheximide } \\
\text { Fluconazole } \\
\text { Ketoconazole } \\
\text { Oligomycin }\end{array}$ & $\begin{array}{l}\text { Cycloheximide } \\
\text { Fluconazole } \\
\text { Ketoconazole } \\
\text { Oligomycin }\end{array}$ & Yes & $\begin{array}{l}\text { kinase complex } \\
\text { Subunit of the SAGA } \\
\text { transcriptional regulatory } \\
\text { complex, }\end{array}$ \\
\hline $\begin{array}{l}\text { SRB8 } \\
\text { (YCR018W) }\end{array}$ & $\begin{array}{l}\text { Atorvastatin } \\
\text { Cycloheximide } \\
\text { Fluconazole } \\
\text { Oligomycin }\end{array}$ & $\begin{array}{l}\text { Atorvastatin } \\
\text { Cycloheximide }\end{array}$ & Yes & $\begin{array}{l}\text { Subunit of the RNA } \\
\text { polymerase II mediator } \\
\text { complex for Pol-II? }\end{array}$ \\
\hline $\begin{array}{l}\text { SSZ1 } \\
\text { (YHR064C) }\end{array}$ & $\begin{array}{l}\text { Cycloheximide } \\
\text { Oligomycin }\end{array}$ & $\begin{array}{l}\text { Cycloheximide } \\
\text { Fluconazole } \\
\text { Ketoconazole } \\
\text { Oligomycin }\end{array}$ & - & $\begin{array}{l}\text { Hsp70 protein; also involved } \\
\text { in pleiotropic drug } \\
\text { resistance via activation of } \\
\text { PDR1 and PDR5 }\end{array}$ \\
\hline $\begin{array}{l}\text { SWI4 } \\
\text { (YER111C) }\end{array}$ & Cycloheximide & $\begin{array}{l}\text { Cycloheximide } \\
\text { Fluconazole } \\
\text { Ketoconazole }\end{array}$ & - & $\begin{array}{l}\text { DNA binding component of } \\
\text { the SBF complex (Swi } 4 p- \\
\text { Swi6p), a transcriptional } \\
\text { activator }\end{array}$ \\
\hline $\begin{array}{l}\text { UME6 } \\
\text { (YDR207C) }\end{array}$ & $\begin{array}{l}\text { Atorvastatin } \\
\text { Benomyl } \\
\text { Cycloheximide }\end{array}$ & $\begin{array}{l}\text { Atorvastatin } \\
\text { Cycloheximide } \\
\text { Fluconazole } \\
\text { Ketoconazole } \\
\text { Oligomycin }\end{array}$ & - & $\begin{array}{l}\text { Key transcriptional regulator } \\
\text { of early meiotic genes, }\end{array}$ \\
\hline $\begin{array}{l}\text { YME1 } \\
\text { (YPR024W) }\end{array}$ & - & $\begin{array}{l}\text { Atorvastatin } \\
\text { Cycloheximide } \\
\text { Fluconazole } \\
\text { Ketoconazole } \\
\text { Oligomycin }\end{array}$ & - & $\begin{array}{l}\text { Mitochondrial inner } \\
\text { membrane i-AAA protease } \\
\text { complex, responsible for } \\
\text { degradation of unfolded or } \\
\text { misfolded proteins }\end{array}$ \\
\hline \multicolumn{5}{|c|}{$\begin{array}{l}\text { Foot note: Sensitivity of } \Delta x x x \text { was compared to wild-type cells. Sensitivity of } \\
\Delta x x x \Delta p d r 1 \Delta p d r 3 \text { was compared to } \Delta p d r 1 \Delta p d r 3 \text {. Different compound concentrations were } \\
\text { used in } \Delta x x x \text { and } \Delta x x x \Delta p d r 1 \Delta p d r 3, \text { as stated in Materials and Methods Table } 2-1 \text {. The } \\
\text { concentrations used in the } \Delta x x x \Delta p d r 1 \Delta p d r 3 \text { strains were adjusted so that the } \Delta p d r 1 \Delta p d r 3 \\
\text { strain grows normally. Genes listed above cause increase in sensitivity to drug(s) in the } \\
\Delta p d r 1 \Delta p d r 3 \text { background. The symbol "-" means } \Delta x x x \text { caused no sensitivity towards } \\
\text { compounds tested in wild-type or extra sensitivity in } \Delta p d r 1 \Delta p d r 3 \text { background, or no } \\
\text { reduction in Pdr5p abundance observed in Western blot. }\end{array}$} \\
\hline
\end{tabular}




\section{Genes which Increase Sensitivity in the double mutant $\Delta p d r 1 \Delta p d r 3$ background}

To investigate if any of the genes identified in the primary screens caused extra sensitivity in double knockout of transcription factors $P D R 1$ and $P D R 3$, we mated the 54 hit strains with strains lacking both $P D R 1$ and PDR3 (genotype: pdr1 $\triangle:$ :

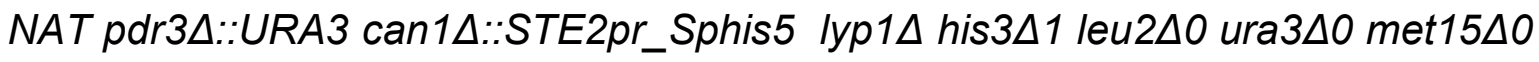
$L Y S 2+)$. The $P D R$ deletion strains were a gift from Namal Coorey; SBS, VUW. The rationale behind this was that gene deletions that conferred extra sensitivity in (already sensitive) the $\Delta p d r 1 \Delta p d r 3$ background strain should in theory, be the genes that are upstream of Pdr1p/Pdr3p and control other compensatory pathways.

We found that most genes that caused sensitivity when deleted by themselves also caused extra sensitivity to one or more drugs when tested in the $\Delta p d r 1 \Delta p d r 3$ background. Furthermore, we found that deletion of CWH43, YDR532C, BMH1, GET1, OPI3, GTR2, YME1, and GCN5 only caused sensitivity in the $\triangle p d r 1 \Delta p d r 3$ background. Deletion of RCY1,KAP123, and ROX3 were synthetic lethal with $\Delta p d r 1 \Delta p d r 3$, while single deletions of these genes individually were viable (Table 4-3). Interestingly, while single $\Delta r c y 1$, and $\Delta r o x 3$ increased sensitivity to compounds tested above (Table 4-3), $\Delta$ kap123 did not cause increased sensitivity in the first spot dilution assays. Examples of gene deletions which conferred sensitivity in this background are shown in Figure 4-7 (gene deletions which conferred sensitivity in both $\Delta x x x$ and $\Delta x x x \Delta p d r 1 \Delta p d r 3$ ), and Figure 4-8 (gene deletions which conferred sensitivity in $\Delta p d r 1 \Delta p d r 3$ background alone). The network of gene deletions that conferred sensitivity in spot dilution assays grouped by GO function is shown in Figure 4-9. 


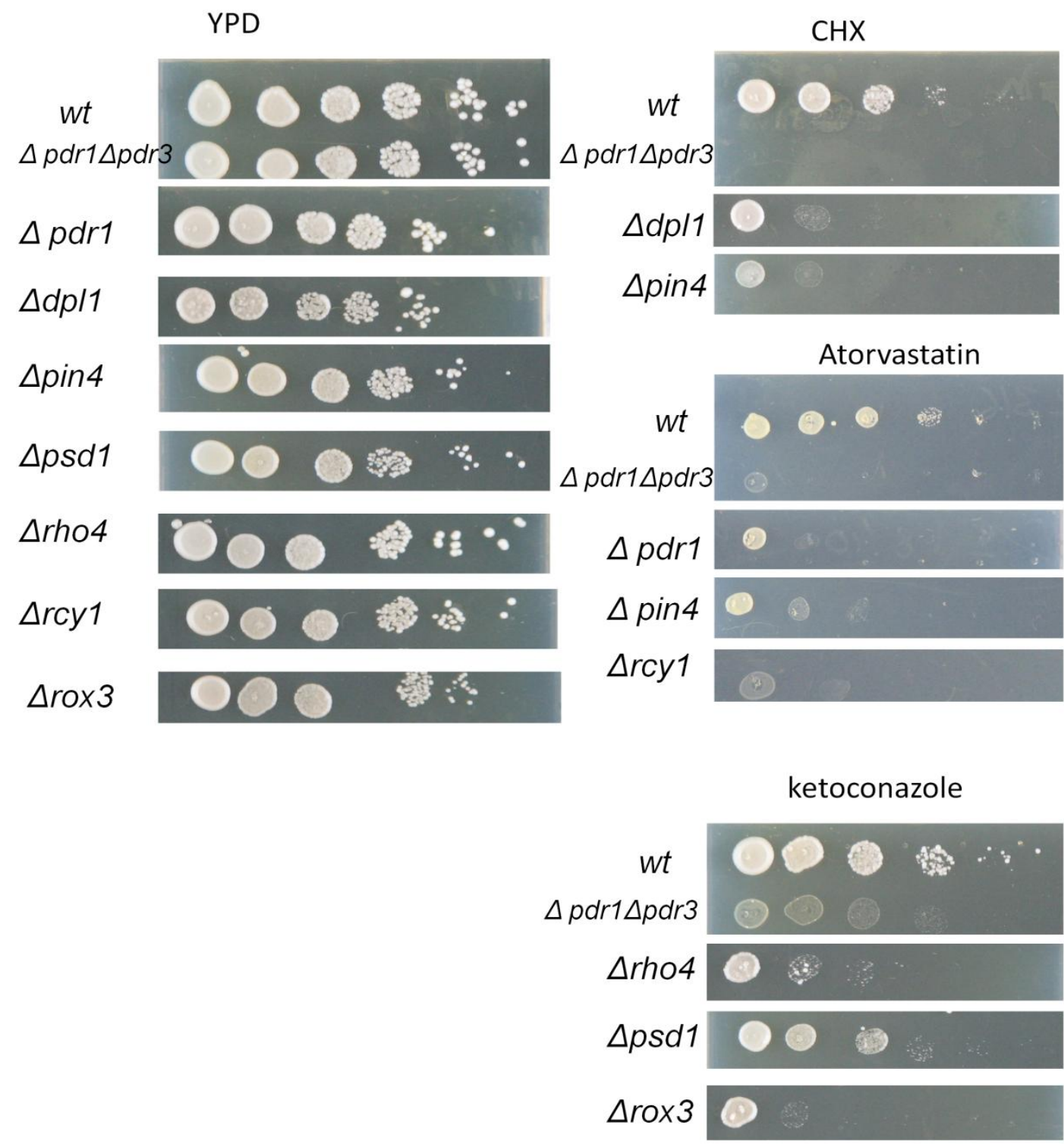

Figure 4-6: Examples of gene deletions from primary screens that conferred sensitivity to compound(s) tested.

Concentrations used in spot dilution assays were listed in Materials and Methods Table 2-1. YPD was used as a control condition. CHX: Cycloheximide 

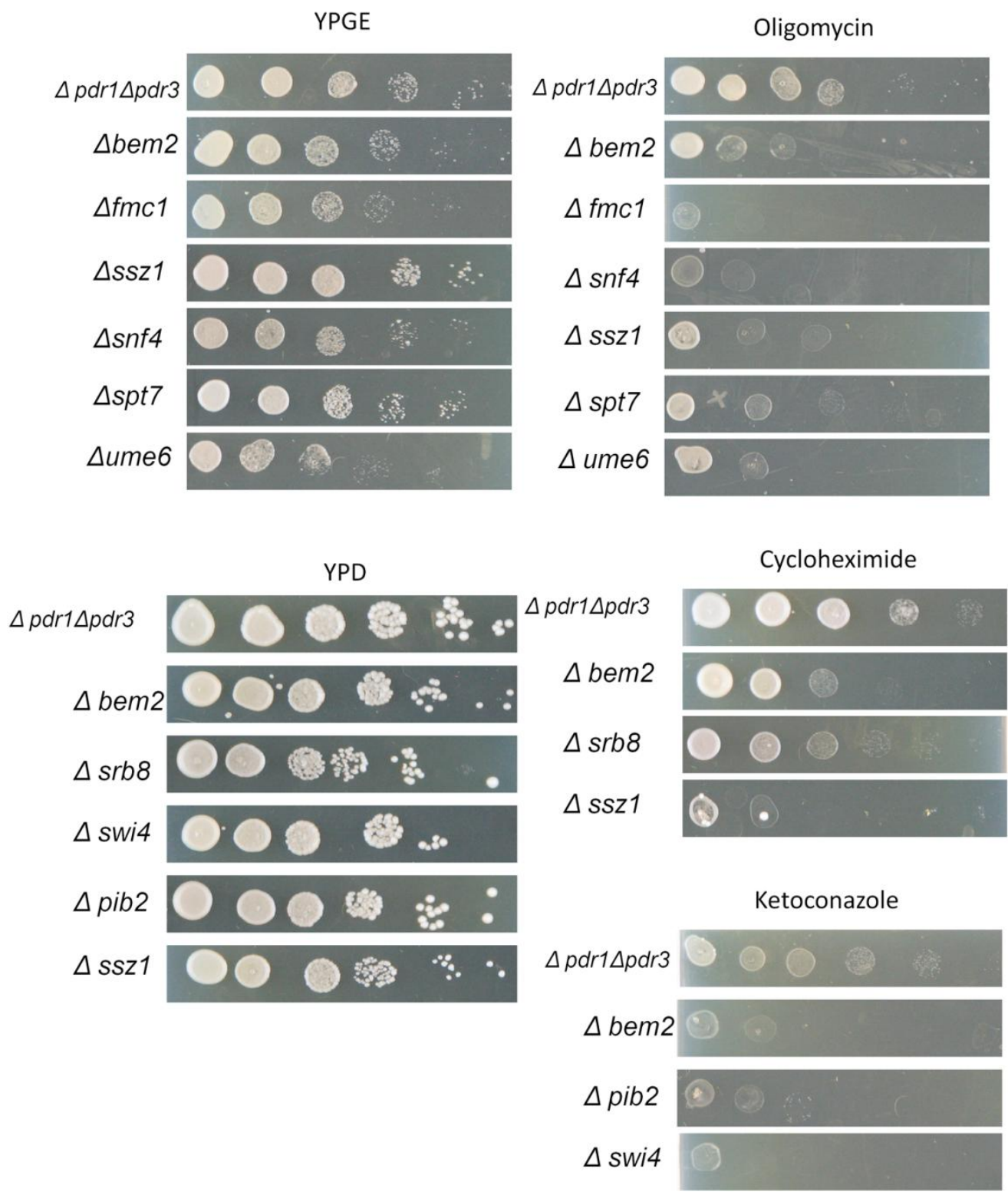

Figure 4-7: Examples of gene deletions which result in an increase in sensitivity toward compound(s) tested as both single deletions $(\Delta x x x)$ and in the $\Delta p d r 1 \Delta p d r 3$ background ( $\Delta x \times x \Delta p d r 1 \Delta p d r 3)$.

The spot dilutions above were carried out with gene deletions in $\Delta p d r 1 \Delta p d r 3$ background. Concentrations used in spot dilution assays were listed in Materials and Methods Table 2-1. YPD was used as a control condition for cycloheximide and ketoconazole. YPGE was used as a control condition for oligomycin. 

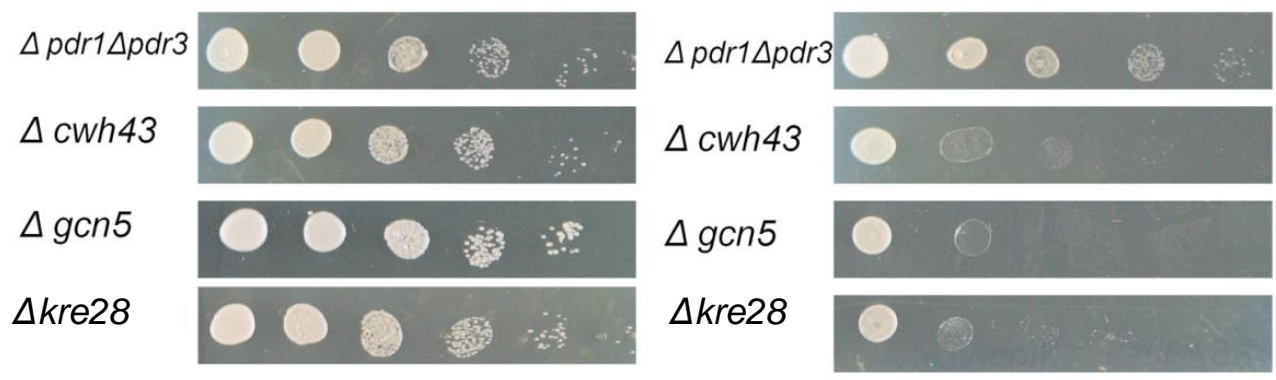

YPD

Ketoconazole
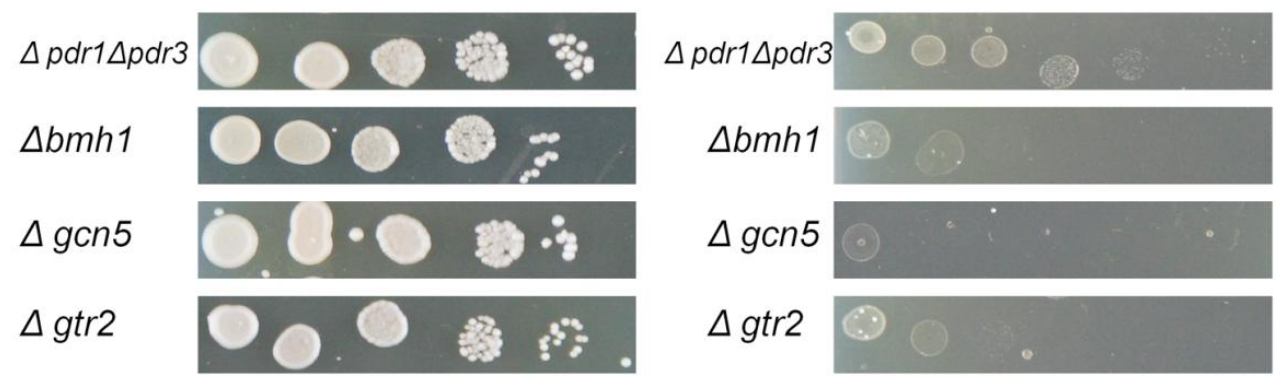

Figure 4-8: Example of gene deletions which result in increase in sensitivity toward compound(s) tested only when in $\Delta p d r 1 \Delta p d r 3$ background.

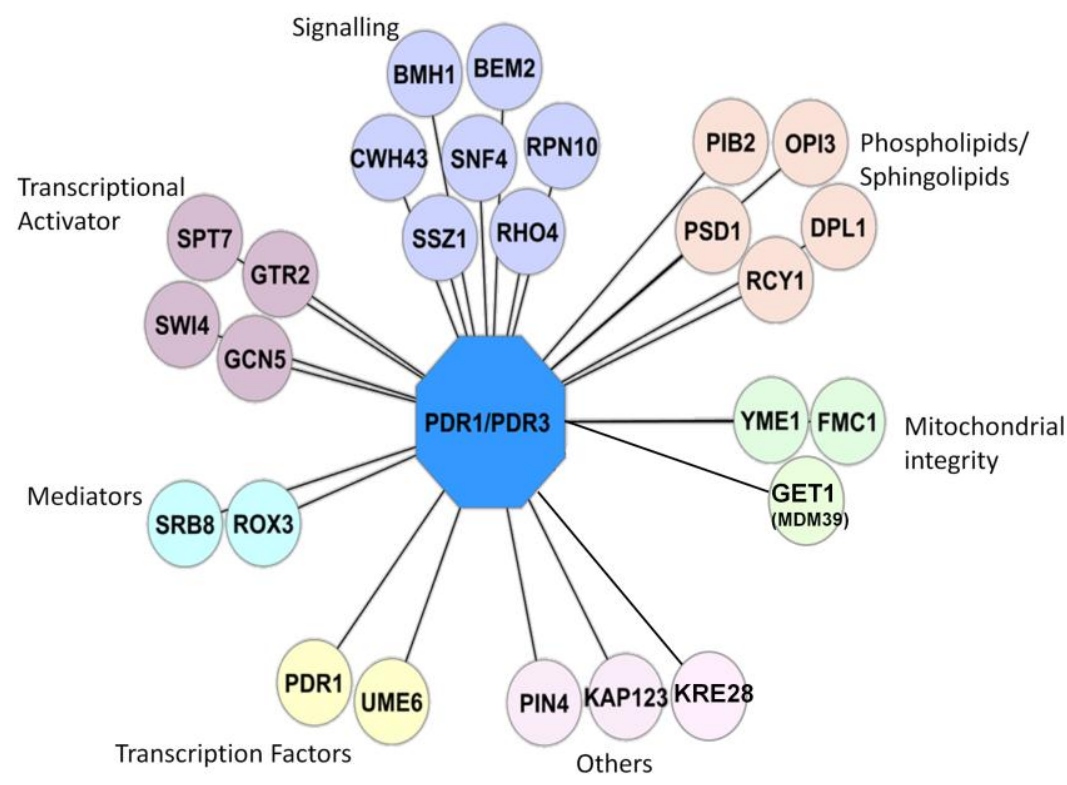

Figure 4-9: Network of genes from PDR secondary screen.

Gene deletions from the primary screens were subjected to spot dilution assay as $\Delta x x x$, and $\Delta x x x \Delta p d r 1 \Delta p d r 3$. Deletions which confer sensitivity toward at least one the six test compounds tested on the genome-wide deletion background are shown here. PDR1/PDR3 are shown as the hub connecting known regulators of the PDR. Each connection line represents a possible regulatory relationship between gene $X X X$ and PDR1/PDR3. 


\section{Gene deletions and effect on Pdr5p abundance}

Twenty-six gene deletions that increased xenobiotics sensitivity were subjected to Western analysis for Pdr5p. This is to investigate if increase in xenobiotic sensitivity in these gene deletion backgrounds was due to changes in Pdr5p abundance (Figure 4-10 and Table 4-3). We found that in addition to $\Delta p d r 1$ and $\Delta p d r 3$, several other genes reduced Pdr5p relative to the Pma1p control, which showed no alteration in abundance on Western blots. These genes were $\Delta$ rox3, $\Delta s p t 7, \Delta g t r 2$, and $\Delta b m h 1$. Table 4-3 also lists other gene deletions that did not result in reduction of Pdr5p abundance measured by Western blot.

Since the expression of another plasma membrane, Pma1p, internal control, did not change upon deletions of the above genes, this suggested that the effect of these various gene deletions was to be specific and not a pleiotropic effect. Of particular interest are those that also group significantly by GO analysis as transcription factors and RNA-polymerase-II mediators, namely ROX3 and SPT7. 

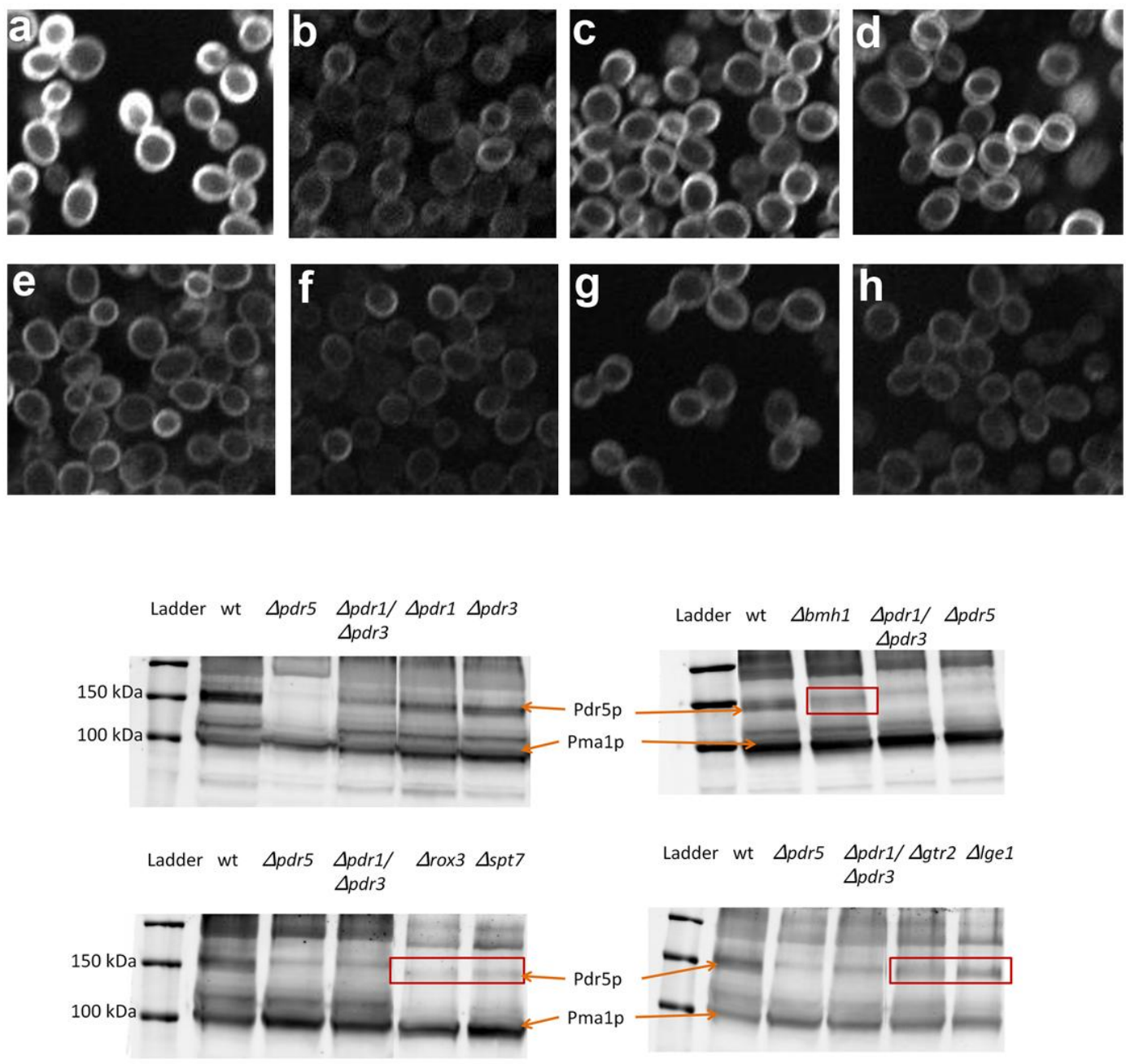

Pdr5p_GFP $\Delta p d r 1 \Delta p d r 3$

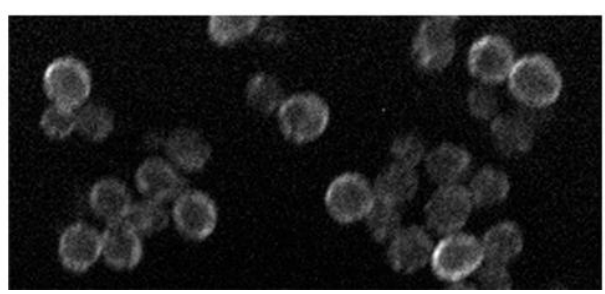

cytoplasmic RFP mCherry $\Delta p d r 1 \Delta p d r 3$

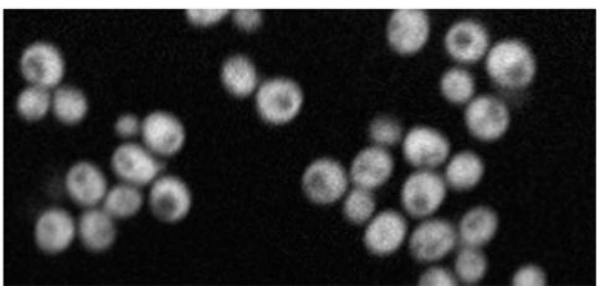

Figure 4-10: Gene deletions that reduce Pdr5p abundance

Images from the primary screen (top panels) and Western blots (middle panels) showing gene deletions which reduced Pdr5p abundance. Top panels a) wild-type; b) $\Delta p d r 1$; c) $\Delta$ pdr3; d) $\Delta b m h 1$; e) $\Delta g t r 2$; f) $\Delta(g e 1$; g) $\Delta s p t 7$; h) $\Delta$ rox3. The cell images were adjusted so that fluorescent intensities can be directly compared. Bottom panel showed Pdr5p-GFP expression in $\Delta p d r 1 \Delta p d r 3$ background with cytoplasmic RFP mCherry internal control. 


\section{Network analysis predicts gene deletions contribute to PDR phenotype.}

From the results reported here, the network of genes that affect the PDR response in S. cerevisiae can be seen to include PDR1 PDR3, as well as gene deletions that showed GO enrichment such as mediators, the SAGA complex, and the genes involved in signalling.

To make this network more comprehensible, and give better idea of how these 26 genes from the secondary validation work together, we literature searched for both physical and genetic interactions among these 26 genes (http://thebiogrid.org/). We then identified the most highly connected genes ("hubs") among these 26 genes (Figure 4-11and Figure 4-12).

We then extended this network of genes affecting the PDR phenotypes by reinterrogating the primary screen in a specific way as follows: we made a list of gene deletions in the published genetic and physical interaction data that interact with PDR1 and PDR3 and with the highly connected "hubs" (http://thebiogrid.org/). This curated list of genes was then used to "screen" the genes that registered in our primary screen but missed the cutoff used in this study for declaring them as "hits". A list of a further 18 gene deletions was obtained that were then tested to see if they showed sensitivity in spot dilution assays or reduced Pdr5p abundance by Western blot. We found that 10 out of 18 gene deletions tested were either showed sensitivity toward compounds tested or decreased Pdr5p abundance in Western blot, or both. The results are shown in Table 4-4.These results suggested that these genes contribute to the PDR network.

Again, we found that most of the genes that displayed sensitivity in the spot dilution assay or reductions of Pdr5p in Western blots were enriched in transcription factors, the SAGA/transcriptional activator and chromatin remodelling (DEP1, NGG1, SPT8, ADA2) by GO analysis. 


\begin{tabular}{|c|c|c|c|}
\hline Gene & $\begin{array}{l}\text { Sensitivity in single } \\
\text { deletion }\end{array}$ & $\begin{array}{l}\text { Sensitivity in } \\
\Delta p d r 1 \\
\Delta p d r 3 \\
\text { background }\end{array}$ & Description \\
\hline $\begin{array}{l}\text { ADA2 } \\
\text { (YDR448W) }\end{array}$ & Cycloheximide & $\begin{array}{l}\text { Not } \\
\text { conclusive }\end{array}$ & $\begin{array}{l}\text { Transcription coactivator, } \\
\text { component of the ADA and } \\
\text { SAGA transcriptional } \\
\text { adaptor/HAT (histone } \\
\text { acetyltransferase) complexes }\end{array}$ \\
\hline $\begin{array}{l}\text { DEP1 } \\
\text { (YAL013W) }\end{array}$ & Cycloheximide & $\begin{array}{l}\text { Fluconazole } \\
\text { Oligomycin }\end{array}$ & $\begin{array}{l}\text { Transcriptional modulator } \\
\text { involved in regulation of } \\
\text { structural phospholipid } \\
\text { biosynthesis }\end{array}$ \\
\hline $\begin{array}{l}\text { ERG3 } \\
\text { (YLR056W) }\end{array}$ & $\begin{array}{l}\text { Atorvastatin } \\
\text { Cycloheximide } \\
\text { Oligomycin }\end{array}$ & no & $\begin{array}{l}\text { C- } 5 \text { sterol desaturase, } \\
\text { catalyzes the introduction of a } \\
\text { C-5(6) double bond into } \\
\text { episterol, a precursor in } \\
\text { ergosterol biosynthesis }\end{array}$ \\
\hline $\begin{array}{l}\text { HEK2 } \\
\text { (YBL032W) }\end{array}$ & no & $\begin{array}{l}\text { Ketoconazole } \\
\text { Oligomycin }\end{array}$ & RNA binding protein \\
\hline $\begin{array}{l}\text { HEX3 (SLX5) } \\
\text { (YDL013W) }\end{array}$ & $\begin{array}{l}\text { Atorvastatin } \\
\text { Oligomycin? }\end{array}$ & $\begin{array}{l}\text { Fluconazole } \\
\text { Ketoconazole }\end{array}$ & $\begin{array}{l}\text { Subunit of the SIx5-SIx8 } \\
\text { SUMO-targeted ubiquitin ligase } \\
\text { (STUbL) complex, }\end{array}$ \\
\hline $\begin{array}{l}\text { HSP82 } \\
\text { (YPL240C) }\end{array}$ & $\mathrm{N} / \mathrm{T}$ & $\mathrm{N} / \mathrm{T}$ & $\begin{array}{l}\text { Hsp90 chaperone required for } \\
\text { pheromone signalling }\end{array}$ \\
\hline $\begin{array}{l}\text { LGE1 } \\
\text { (YPL055C) }\end{array}$ & no & no & Protein of unknown function \\
\hline $\begin{array}{l}\text { MNN10 } \\
\text { (YDR245W) }\end{array}$ & $\begin{array}{l}\text { Atorvastatin } \\
\text { Benomyl }\end{array}$ & $\begin{array}{l}\text { Atorvastatin } \\
\text { Cycloheximide } \\
\text { Fluconazole } \\
\text { Ketoconazole }\end{array}$ & $\begin{array}{l}\text { Subunit of a Golgi } \\
\text { mannosyltransferase complex. }\end{array}$ \\
\hline $\begin{array}{l}\text { NGG1 } \\
\text { (YDR176W) }\end{array}$ & $\begin{array}{l}\text { Atorvastatin } \\
\text { Cycloheximide } \\
\text { Ketoconazole } \\
\text { Oligomycin }\end{array}$ & $\mathrm{N} / \mathrm{T}$ & $\begin{array}{l}\text { Transcriptional regulator; } \\
\text { component of transcriptional } \\
\text { adaptor and histone } \\
\text { acetyltransferase complexes, } \\
\text { the ADA complex, the SAGA } \\
\text { complex, and the SLIK complex }\end{array}$ \\
\hline $\begin{array}{l}\text { SPT8 } \\
\text { (YLR055C) }\end{array}$ & $\begin{array}{l}\text { Atorvastatin } \\
\text { Fluconazole } \\
\text { Ketoconazole }\end{array}$ & $\begin{array}{l}\text { Fluconazole } \\
\text { Ketoconazole }\end{array}$ & $\begin{array}{l}\text { Subunit of the SAGA } \\
\text { transcriptional regulatory } \\
\text { complex but not present in } \\
\text { SAGA-like complex }\end{array}$ \\
\hline
\end{tabular}




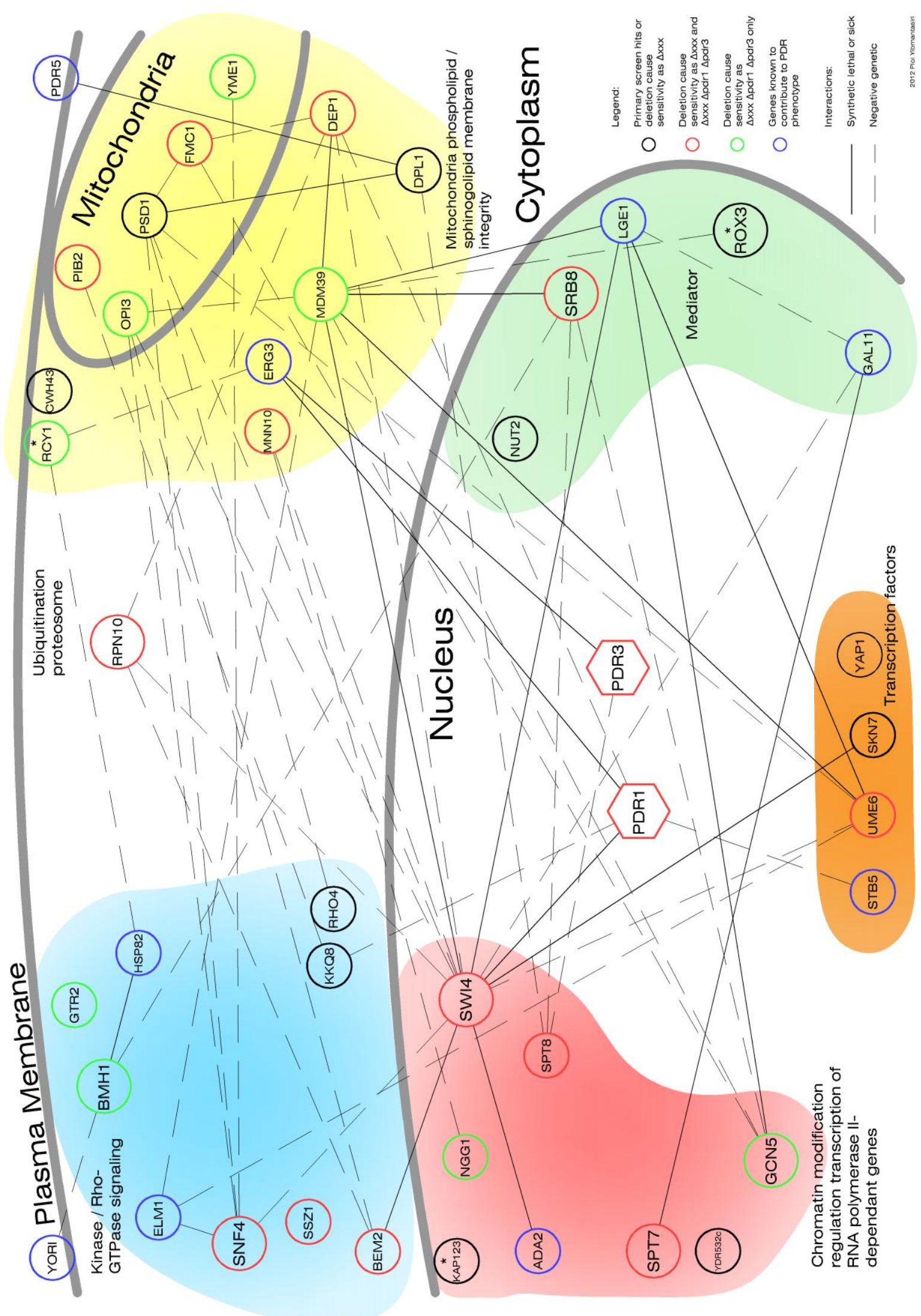

Figure 4-11: Published genetic (http://thebiogrid.org/) networks among our screen results and some of the known PDR-contributing genes. 


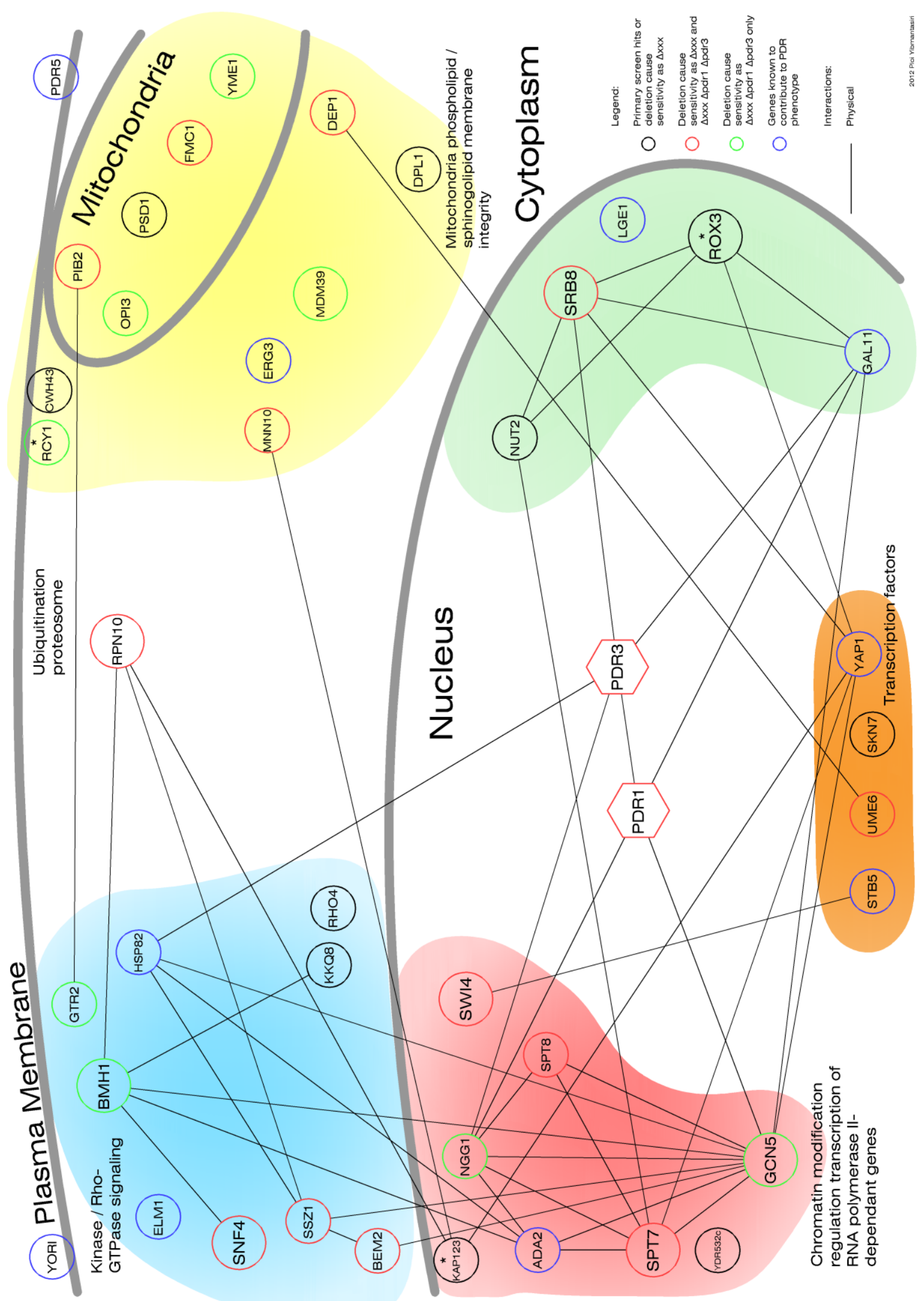

Figure 4-12: Published physical interaction (http://thebiogrid.org/) networks among our screen results and some of the known PDR-contributing genes. 


\section{Discussion}

Section I: Methodologies, validation and limitations of the reporter screens

(a). Reporters for Investigating Possible PDR Modulators: Primary Screen with Pdr5p and Yor1p Reporters

We developed a Green Fluorescent Protein (GFP)-based reporter system utilizing Pdr5p, and Yor1p in order to identify other possible modulators of the PDR response in addition to the known regulators Pdr1p/Pdr3p.

When we screened the LOPAC library with our GFP reporters (Pdr5p, Snq2p, and Yor1p), we found that some compounds with different analogues of some compounds activate different sets of pumps. For example, phenylalanine chloromethyl ketone, the Z-L analogue activated all three pumps, while the N-pTosyl-L analogue activated only Snq2p, and Yor1p. There are two explanations for this. First is that, different analogues interact differently with different pumps. The second explanation is that the N-p-Tosyl-L analogue activates Yrm1p, whose recognition sites are found only in SNQ2 and YOR1 promoter region and not in $P D R 5$, whilst the Z-L analogue activates TFs for all three pumps. This result is the first evidence that the PDR phenotype has back up pathways which are independent of Pdr1p/Pdr3p.

The results reported as "hits" in this chapter are gene deletions that resulted in a constant ABC-GFP reporter fluorescence level in the presence of xenobiotic compounds that otherwise would result in increased expression of ABC-GFPreporter. Gene deletions that scored this way but that also caused the UPRE-GFP assay to switch on were eliminated as false positive. Hits were recorded when a particular gene deletion scored in 4 out of the 5 screens of which 3 repeats were for Pdr5p-GFP and 2 repeats for Yor1p-GFP. Although Pdr5p and Yor $1 p$ are Pdr1p/Pdr3p targets, their expression and degree of dependency on these two transcription factors are different. PDR5 is more dependent on Pdr1p/Pdr3p than YOR1 (Kolaczkowska and Goffeau 1999). PDR5 also contains recognition sequences belonging to other transcription factors such as Yap1p and Cad1p. These latter both contribute to the oxidative stress response (Miyahara, Hirata et al. 1996). Expression of YOR1 can also be controlled by Yrr1p another transcription factor known to contribute to the PDR phenotype. Because we do not 
know the extent of the PDR network and therefore the extent of commonality of modulators, using these two pumps as reporters increased the chances of identifying common genes that affect both $A B C$ transporters.

(b). Does the primary screen specifically identify genes needed for increased expression of $A B C$ transporters in the plasma membrane?

Some positive controls of expected hits are built into the reporter screens, namely that deletions of PDR1 and PDR3 were expected hits and indeed were obtained from all the screens. Some of the genes have also been shown to effect drug resistance such as PSD1, and SSZ1 also were identified as hits (Hallstrom, Katzmann et al. 1998). This was proof-of-principle that using $A B C$ transporter levels in the plasma membrane is a valid screening methodology.

\section{(c). Lack of appearance of putative positive controls}

It is noteworthy that some positively controlled genes did not come up as hits in our screen. These were GAL11, YRR1, and ELM1. Gal11p is a subunit of the RNA polymerase II mediator complex and is known to be a mediator required for Pdr1p and Pdr3p function in the PDR response (Thakur, Arthanari et al. 2008; Shahi, Gulshan et al. 2010) and to have physical interactions with both transcription factors. In the current studies, some mediators such as ROX3 and SRB8 scored as hits as expected. The reason why GAL11 did not appear as a hit neither in the primary screen nor in the spot dilution assays might be due to alternative pathways and/or replacement of Gal11p by other mediators. The possible alternative pathways will be discussed below.

YRR1 is $\mathrm{Zn}_{2} \mathrm{Cys}_{6}$ zinc-finger transcription factor and was shown to contribute to multidrug resistance via binding to the promoter of Snq2p and Yor1p. A mutant in YRR1 was shown to confer resistance to a variety of compounds such as revoromycin A, 4-NQO, and oligomycin (Cui, Shiraki et al. 1998). The YRR1 deletion was expected to record as a hit in the Yor1p reporter screen but not in the Pdr5p reporter screen. However, we did not get YRR1 as a hit in the Yor1p reporter screen against atorvastatin. One explanation for this might be that Yrr1p is not needed in the atorvastatin response or it could be that Yrr1p acts downstream of Pdr1p/Pdr3p and the requirement of Yrr1p can be bypassed as long as Pdr1p/Pdr3p are present. YRR1 is autoregulated and contains the PDRE 
in its promoter. It has been suggested that Pdr1p controls the expression of YRR1 and might use Yrr1p as a way to amplify its signal to the overlap targets between Pdr1p/Pdr3p and Yrr1p (Zhang, Cui et al. 2001; Gavin, Aloy et al. 2006).

The lack of appearance of some genes expected as positive controls means that the set of genes recorded as hits will be a minimal list only, suggesting some other condition may also necessary in our screens to pick up all the genes involved. Another explanation is that these gene functions can be bypassed by alternative parallel pathways.

\section{(d). Validation of primary screen hits using spot dilution growth assays}

All the 54 primary screen hits were subjected to spot dilution assays with various compounds to make sure that the 54 deletion genes (Table 4-3, Figure 4-6, Figure 4-7, and Figure 4-8) we obtained as hits were not specific to the atorvastatin response alone. It must be emphasised that spot dilution assays measure growth sensitivity rather than Pdr5p abundance as is measured with the PDR5-GFP and Western blot analyses. Growth analysis therefore measures the overall ability of cells to deal with xenobiotics, which may be affected by factors other than Pdr5p abundance, and the ability to pump out the xenobiotics. Additional factors might include other $A B C$ pumps such that gene deletions in the spot dilution assays might not be affecting Pdr5p but might be increasing sensitivity towards compounds because of decrease in abundance of other pumps such as Snq2p, Yor1p. Relating to Pdr5p, however, $\Delta b m h 1, \Delta g t r 2, \Delta p d r 1, \Delta r o x 3, \Delta s p t 7$ all showed reduction in Pdr5p abundance in Western blots (Table 4-3) these genes will be further discussed below in the Western blot results. With these reservations, we conclude that the spot dilution assays did in general validate the primary screen.

In the spot dilution assays 26 gene deletions out of the 54 identified in the primary screens showed some form of xenobiotic sensitivity. Of these, 5 genes displayed sensitivity when deleted as single genes $(\Delta x x x), 10$ displayed sensitivity as both single deletion and extra sensitivity in $\Delta p d r 1 \Delta p d r 3$ background ( $\Delta x x x \Delta p d r 1 \Delta p d r 3)$. Eight genes showed sensitivity only in the absence of $P D R 1$ and PDR3 ( $\triangle x x x$ $\Delta p d r 1 \Delta p d r 3$ ) and 3 genes displayed negative genetic interactions when deleted in $\Delta p d r 1 \Delta p d r 3$ background ( $\Delta x x x \Delta p d r 1 \Delta p d r 3$, did not grow in rich media) (Table 4-3). It can be concluded that some gene deletions caused extra sensitivity in the 
$\triangle p d r 1 \Delta p d r 3$ background, which by definition must be a PDR response that is different to that controlled, by $P D R 1$ and PDR3.

When spot dilution assays were carried out to test for xenobiotic sensitivity in these single deletion backgrounds, we were surprised to see that $\Delta p d r 1$, a deletion in a main PDR transcription factor, only caused sensitivity to atorvastatin. By contrast, $\Delta$ ume 6 was sensitive to nearly all xenobiotics including atorvastatin, similarly for $\Delta s r b 8$, and $\Delta s p t 7$. Some genes such as $\Delta r h o 4$, and $\Delta b e m 2$ which have no known function on transcriptional activity themselves conferred sensitivity to a greater variety of compounds than did $\Delta p d r 1$. Deletion of PDR3 alone did not show sensitivity toward any compounds in the concentrations used. Perhaps, this is because Pdr1p and Pdr3p functions overlap and compensate for each other's activity. Our results clearly suggest that PDR1 and PDR3 are not the sole contributors to the PDR phenotype and there are upstream modulators required for a PDR phenotype to be fully developed.

Other noteworthy points are, first, that genes that are involved in a pathway that is affected by the xenobiotics will show sensitivity toward those xenobiotics when deleted (Table 4-3). For example, gene deletions that conferred sensitivity toward oligomycin by inhibiting ATPsynthase in the electron transport chain tend to involve in mitochondrial function, and metabolism (SNF4, YME1, and OP/3). Again, this result reinforces the importance of chemical-genetic interactions as a powerful tool to identify the drug-target pathway and drug mode-of-action as previously mentioned in Chapter 3. In that chapter, we concluded that neo affected vacuole formation and the endocytosis pathway as indicated by enrichment of ESCRT complex in the neo chemical-genetic profile. Secondly, gene deletions, which displayed sensitivity toward multiple xenobiotics, tend to involve signal transduction and transcriptional control (Table 4-3) (e.g. BEM2, RHO4, GCN5, SPT7, SRB8, and UME6). This result strongly suggests an involvement of upstream signalling pathway in PDR response.

\section{(e). Western blot analysis of reporter protein abundance.}

Gene deletions that reduced the abundance of Pdr5p were $\Delta p d r 1$ and $\Delta p d r 3$, $\Delta$ rox3, $\Delta s p t 7, \Delta g t r 2, \Delta b m h 1, \Delta l g e 1, \Delta s p t 8, \Delta$ dep1, $\Delta h s p 82$. These reductions were specific to the particular gene deletions and not a pleiotropic effect as Pma1p, 
another plasma membrane protein, displayed no alteration as a function of these gene deletions. With the exception of $\Delta h s p 82$, all of these gene deletions appeared in the list of hits in the primary screen (Pdr5p-GFP/Yor1p-GFP) albeit $\Delta l g e 1$ and $\Delta$ spt8 at a slightly lower confidence level in only 2 screens out of 3 and $\triangle d e p 1$ appearing only in the Pdr5p-GFP screen. All of these genes, except LGE1, are transcription factors, have role in transcriptional activators or as chromatin remodelling complexes affecting transcription. Such observations lead to the suggestion that the PDR phenotype involves a specialised transcriptional regulatory unit. The fact that $L G E 1$, whose function is unknown and HSP82 also appear in this list of gene deletions suggest they must have an allied function. The notion that the PDR response involves a specialised transcription unit as distinct from say simple interaction of xenobiotics with Pdr1p/Pdr3p transcription factors is discussed further below when evidence of signalling gene involvement is also taken into account.

It is of note that $\Delta \mathrm{pdr} 1$ or $\Delta \mathrm{pdr} 3$ alone reduced but did not abolish the amount of Pdr5p as seen by the Pdr5p/Yor1-GFP intensity screen and from Western blots (Figure 4-10). Deletion of $P D R 1$ had a stronger effect on the reduction of expression of $P D R 5$ than did the deletion of PDR3. This effect is due to the fact that PDR5 expression is known to be more Pdr1p-dependent than Pdr3p (see Introduction) and that Pdr1p also has transcriptional control over the expression of PDR3. However, the combined deletion of both PDR1 and PDR3 reduced cellular abundance of Pdr5p to non-detectable levels by Western Blot but still could be detected using PDR5-GFP in the $\Delta p d r 1 \Delta p d r 3$ background (Figure 4-10). The difference might be because Western Blots are not as sensitive as the GFP intensity quantitation in the microscopy screen.

\section{Section II: Gene deletions that show negative genetic interaction with $\Delta p d r 1 \Delta p d r 3$}

A number of gene deletions appeared as negative genetic interactions with $\Delta p d r 1 \Delta p d r 3$ suggesting that the deletions are components of a different pathway to that controlled by PDR1/PDR3. As an example, supporting this interpretation $\Delta r o x 3$ resulted in negative genetic interaction with $\Delta p d r 1 \Delta p d r 3$ as well as reducing Pdr5p in the Western blot analysis while deletion of genes in the general SAGA complex such as SPT7 and GCN5 did not. However, deletion of other mediators 
such as SRB8 and GAL11 which directly interact with Pdr1p/Pdr3p (Thakur, Arthanari et al. 2008; Shahi, Gulshan et al. 2010) did not result in negative genetic interaction with $\Delta p d r 1 \Delta p d r 3$ implying that $R O X 3$ mediates a compensatory pathway for PDR1/PDR3 different to the Gal11p involvement.

We also found that deletion of $R C Y 1$ resulted in a negative genetic interaction in the $\Delta p d r 1 \Delta p d r 3$ background suggesting that Rcy1p compensate for the loss of function of Pdr1p/Pdr3p. Rcy1p is an F-box protein involved in recycling plasma membrane proteins and signal transduction (Craig and Tyers 1999). Since Rcy1p is involved in recycling plasma membrane protein involving SNAREs and endocytosis, deletion of $R C Y 1$ would have an effect on the localisation of any signal receptors on plasma membrane as well as the $A B C$ transporters. Preventing internalisation of receptors and recycling of transporters might ablate a putative signal from toxic compounds to Pdr1p/Pdr3p use. This would result in a build-up of lethal toxic metabolites inside the cells (Wolfger, Mahe' et al. 1997; Rogers, Decottignies et al. 2001; Jungwirth and Kuchler 2006; Sipo and Kuchler 2006; Kuchler and Schüller 2007; Paumi, Chuk et al. 2009). Rcy1p function in endocytosis mi

Negative genetic interactions observed between $\Delta k a p 123$ and $\Delta p d r 1 \Delta p d r 3$ was by comparison unexpected because while deletion of both $R O X 3$ and $R C Y 1$ alone result in increased sensitivity towards many compounds tested, deletion of KAP123 alone did not result in increase of sensitivity toward any compounds tested. KAP123 encodes for karyopherin beta, which mediates nuclear import of ribosomal proteins prior to assembly into ribosomes and is involved in import of histones H3 and H4 (Mosammaparast, Guo et al. 2002; Sydorskyy, Dilworth et al. 2003).

The negative genetic interaction might be due to the fact that Kap123p is required for mediating nuclear import of many proteins. KAP123 shows genetic interaction with the essential gene PSE1, where a double mutant $\triangle$ kap123 $\triangle p s e 1$ blocks mRNA export from the nucleus. PSE1 encodes another karyopherin/importin that interacts with the nuclear pore complex and acts as a nuclear import receptor for specific proteins, including Pdr1p, Yap1p, Ste12p, and Aft1p (Seedorf and Silver 1997; Delahodde, Pandjaitan et al. 2001; Isoyama, Murayama et al. 2001; Ueta, 
Fukunaka et al. 2003). All of these proteins are transcription factors. Pdr1p and Yap1p as discussed previously are involved in multidrug resistance, Ste12p is transcription factor activating the kinase-signalling cascade controlling growth and mating and Aft $1 \mathrm{p}$ is an iron-responsive transcription factor.

Though the negative genetic interactions reported here resulted in a no-growth phenotype, tetrad dissections need to be conducted to confirm the synthetic lethality of these genes with $\Delta p d r 1 \Delta p d r 3$.

\section{Section III. Signalling pathway related genes that were identified by the screens}

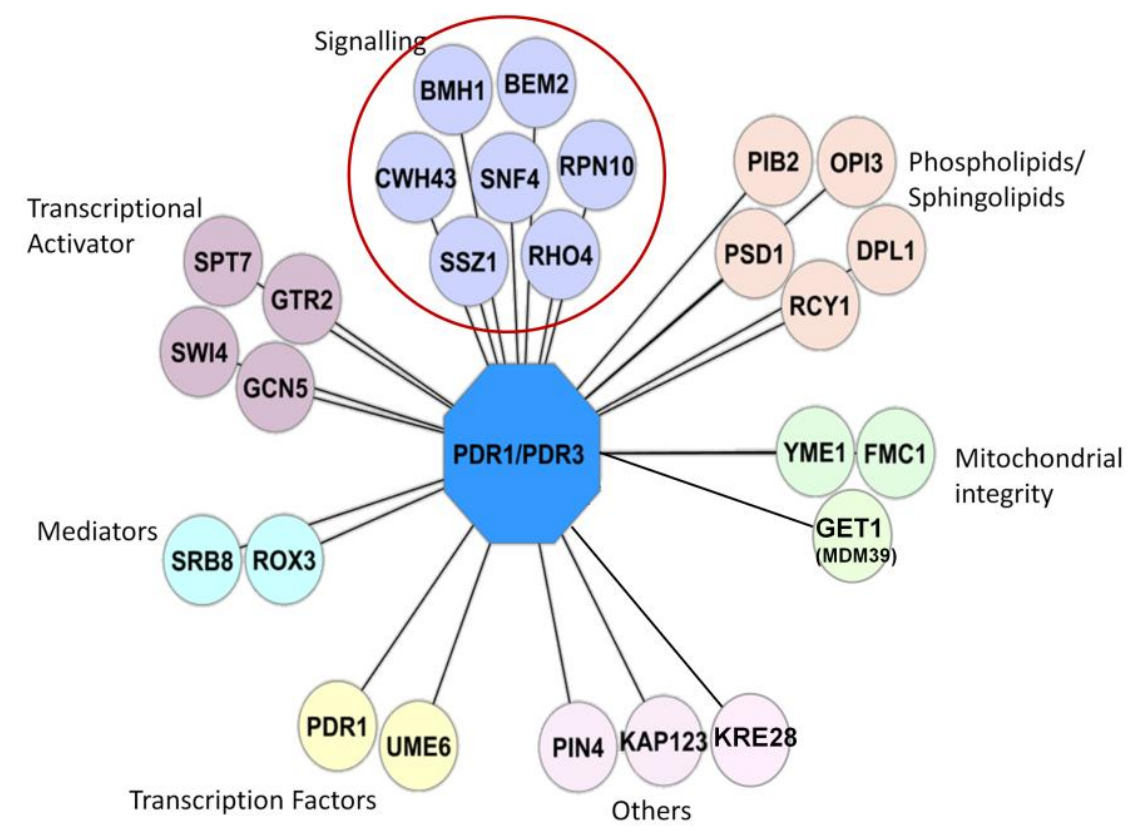

Figure 4-13: Signalling genes in the network of genes for PDR secondary validation

Genes involved in signalling pathways, such as Rho-GTPase, Ras, and MAPK were also noticeably enriched in our screens (Figure 4-13). This finding complements our LOPAC screen result in which many of the compounds that upregulated our $\mathrm{ABC}$-reporters are also known signalling and kinase inhibitors (listed below). Similar complementary results have been observed in $C$. albicans. LaFayette et al. (2010) showed that protein kinase inhibitors in a LOPAC screen which have little toxicity on their own, enhanced the toxicity of fluconazole in $C$. albicans, leading them to propose that kinase signalling regulates multidrug resistance in this pathogenic yeast species (LaFayette, Collins et al. 2010). These 
data combined with the screening results reported here strongly suggest that kinase-signalling pathways are involved in controlling the PDR phenotype.

The observations from both LOPAC and PDR screens further support the notion that there are alternative pathways contributing to the PDR phenotype beside PDR1 and PDR3. These compounds (Table 4-5) might either amplify the "signal" of Pdr1p/Pdr3p or relay the signal in the presence of xenobiotics, or may be involved as general stress responders when PDR1 and PDR3 are absent.

\section{Table 4-5: LOPAC compounds that activate PDR reporters and inhibit signalling-related pathways or molecules}

\section{Compounds}

Farnesylthiosalicylic acid

3-alpha,21-dihydroxy-5-alphapregnan-20-one

Dequalinium analog, C-14 linker

$\mathrm{N}$-phenylanthranilic acid

7-cyclopentyl-5-(4-phenoxy) phenyl7H-pyrolo

DL-stearoylcarnitine chloride

SU 6656

U0126

\section{Mode-of-action}

Non-toxic Ras inhibitor

Positive allosteric modulator of GABA-A receptors

Protein kinase C-alpha (PKC-alpha) inhibitor

$\mathrm{Cl}$ - channel blocker

Potent and selective Ick (src family tyrosine kinase) inhibitor.

Protein kinase $\mathrm{C}$ (PKC) inhibitor

Selective Src family kinase inhibitor

Specific inhibitor of MEK1 and MEK2 (MAP kinase kinase; MAPKK)

All genes that are involved in the signalling category except $\mathrm{RHO} 4$ upregulated the Pdr5p (and Yor1p) pumps independent of Pdr1p/Pdr3p function.

Most of the genes in this category seen in the primary screen are involved in RhoGTPase activating kinases (BEM2, RHO4, SNF4), PKA, Ras-MAPK (BMH1). GO analysis performed on the gene deletions that caused sensitivity in the spot dilution assay showed significant enrichment in genes involved in enzyme activation and protein binding. These genes are BEM2, BMH1, and SNF4 (p-value 0.03599 ). This adds to the suggestion that a signalling pathway, probably kinase proteins (RAS/ MAPK), are required to relay the xenobiotic information sensed at the plasma membrane to the Pdr1p/Pdr3p in the nucleus. 
PKC and MAPK kinases are involved in the regulation of many stress responses and transcription factors such as Msn2p, Skn7p are involved in osmolarity, general stress, salt stress and oxidative stress (Ikner and Shiozaki 2005). However, Ras and PKC have not yet been shown to regulate the PDR response. It is likely that PKC and MAPK can also activate Pdr1p/Pdr3p directly or via other stress transcription factors. For example, Ssz1p is shown to positively regulate the function of Pdr1p but not Pdr3p. It also regulates PDR1-dependent PDR5 and YOR1 expression (Hallstrom 1998). However, the exact mechanism by which cytoplasmic Ssz1p could control Pdr1p in the nucleus is not clear. Hallstrom and Moye-Rowley 1998 suggested that there might be a signalling pathway between Ssz1p and Pdr1p. Gavin et al 2006 showed that Ssz1p physically interacts with Bem2p, a Rho GTPase activating protein (Gavin, Aloy et al. 2006). It is likely that Bem2p relays the regulatory signal from Ssz1p to the subunit of chromatin modification complexes, Gcn5p with which it physically interacts (Lee, Sardiu et al. 2011).

Another mechanism by which signalling could contribute to PDR is by providing backup mechanisms to detoxify xenobiotics. It is possible that Cwh1p and Bmh1p will play this role. Our reasoning is as follows: Cwh1p is a putative sensor/transporter, and deletion of this gene is synthetic lethal with $\Delta p k c 1$, suggesting that Cwh1p is involved in PKC1 signalling. Protein Bmh1p is a 14-3-3 protein that in yeast is thought to function in signal transduction, via Ras/MAPK signalling (Burbelo and Hall 1995; Bruckmann, Hensbergen et al. 2007). We propose that Cwh1p might sense the signal and relay it to Bmh1p. This Bmh1p could be a signal switcher acting to distinguish the signalling response in cells encountering $A B C$-transporter substrates from those that do not. Bmh1p also plays a role in vesicle transport activating the process of compartmental detoxification where toxins are sequestered into the vacuole. In the presence of xenobiotics, Bmh1p may relay signals via Ras/MAPK which autophosphorylates the transcription factors or chromatin modification network and activates the classic PDR response upregulating pumps or other stress response transcription factors such as Msn2p. and Skn7p. Both of these transcription factors have recognition sequences in the promoter regions of both PDR5 and SNQ2 as well as 
other major facilitators (Harbison, Gordon et al. 2004; Venters and Pugh 2009; Biggin 2011).

Section IV: Genes involved in RNA-transcription activation (transcription factors, mediator subunits, chromatin modification complexes)

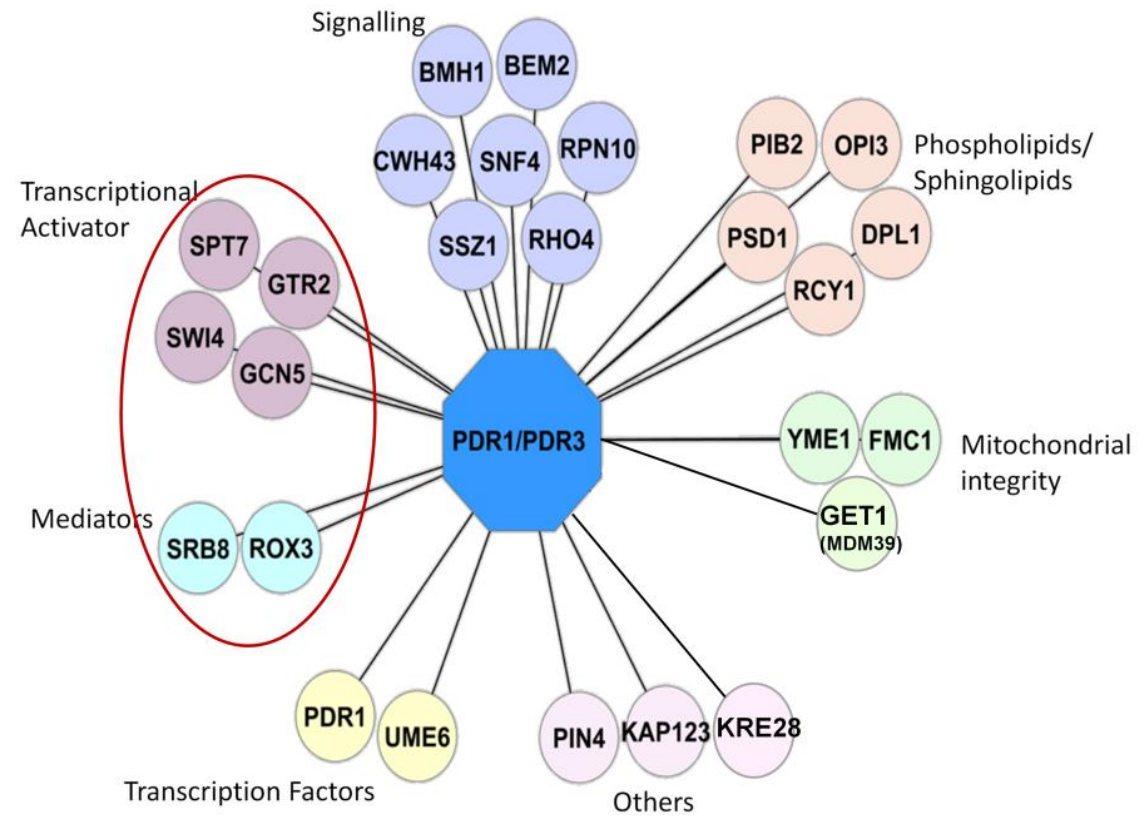

Figure 4-14: transcriptional controlling genes in the network of genes for PDR secondary validation

Additional genes that work independently of PDR1 and PDR3 but that also contribute to the PDR phenotypes were revealed quite unexpectedly. These genes mainly involve transcriptional regulation, either as transcription factors, mediators, via SAGA transcriptional regulatory complex, or in chromatin modification, and seemed to be activated in response to stress. GO function analysis of the genes shown in Table 4-2 using the SGD GO analysis tool (www.yeastgenome.org) showed significant enrichment in transcriptional activity and co-activation which were PDR1, PDR3, UME6, SWI4, SKN7, RSF2, RTG1, ROX3, SRB8, SSN2, and GCN5.

Out of these genes, 3 of them (ROX3, SRB8 and SSN2) function as subunits of the RNA polymerase II mediator complex. Srb8p physically interacts with Pdr3p and deletion of SRB8 $(\Delta s r b 8)$ renders cells that have lost the mitochondrial genome ( $\rho^{0}$ cells) to become sensitive to cycloheximide (Shahi, Gulshan et al. 2010). We found that deletion of SRB8 also resulted in hypersensitivity in strains 
with intact mitochondrial genome $\left(\rho^{+}\right)$and not just in $\rho^{0}$ cells as reported previously (Shahi, Gulshan et al. 2010), suggesting that Srb8p might have more roles in the PDR phenotype than anticipated.

In the primary screen of deletions that prevented up-regulation of ABC-pumps, there were 7 regulatory transcription factors (PDR1, UME6, SKN7, PDR3, HMS2, RTG1 and RSF2 see Figure 4-4) all of which have potential in controlling the PDR response. However, only deletion of PDR1 and UME6 resulted in an increase in growth sensitivity towards one or more of the 6 compounds at the concentration we tested. Deletion of PDR1 caused a reduction of Pdr5p in the Western blot assay while $\Delta$ ume6 did not. The presence wild-type copy of PDR1 PDR3 might mask the effect of these transcription factor gene deletions, and /or they might be regulated by Pdr1p/Pdr3p which would explain why no additional sensitivity to the compounds was seen.

Ume6p is key transcriptional regulator of early meiotic genes. Deletion of UME6 causes a decrease in resistance toward many compounds such as rapamycin, ethanol, cycloheximide, sulfometuron methyl, lovastatin (www.yeastgenome.org) but the link between UME6 and drug resistance has not been shown previously.

Deletion of UME6 ( $\triangle$ ume6) resulted in a more severe growth defect toward many more compounds than did deletion of PDR1. In fact $\Delta$ ume6 $\Delta p d r 1 \Delta p d r 3$ was more sensitive towards all the compounds tested than $\Delta p d r 1 \Delta p d r 3$. However, $\Delta$ ume6 did not cause a reduction in Pdr5p as detected by Western blot analysis probably because Ume6p controls other ABC pumps than Pdr5p. Indeed Ume6p has a recognition sequence in front of the YOR1 promoter (Harbison, Gordon et al. 2004).

Already discussed above are other major components of this group namely chromatin modification complexes SAGA/ADA subunits as well as subunits of the RNA polymerase II mediator complex and a transcriptional activator cimplex (SPT7, GCN5, ROX3, SRB8, and SWI4) where we discussed compelling evidence that their involvement is PDR-specific and not a global response.

The recognition site of Swi4p has been found in the promoters of SNQ2 and PDR5, and some of the major facilitators. Skn7p which is controlled by the SWI complex 
and MAPK also has recognition sites on SNQ2, PDR5 and YOR1 (Harbison, Gordon et al. 2004; Workman, Mak et al. 2006; Venters and Pugh 2009). Although, we cannot exclusively said that Swi4p control these pumps based on the recognition sites on the promoters alone, it give us a good hint of what might actually happen. The SAGA, SWI/SNF chromatin modification complex though often global in function may provide cells with a specific minor backup pathway for Pdr1p/PDr3p. We propose that in the absence of the major regulator Pdr1p/Pdr3p these minor regulator components become essential. Both SPT8 and SWI4 show negative genetic interactions with GAL11, strongly supporting our proposal that the SAGA and SWI complexes provide cells with an independent alternative pathway to achieve the PDR phenotype, maybe by bypassing the requirement of Gal11p or Pdr1p/PDr3p altogether. This could explain why GAL11 does not appear as a hit in our primary screen or spot dilution as expected. This hypothesis also explains the observed results that most of the strains containing deletion of genes in this category were sensitive to xenobiotics and become hypersensitive in $\Delta p d r 1 \Delta p d r 3$ background.

Venters and Pugh 2009 found that spatial assembly of the transcription machinery and chromatin regulators at promoters can result in altered gene expression dependant on the signal received (Venters and Pugh 2009) (Figure 4-15). It seems likely to us that interactions of such major and minor contributors with spatial variation could be the basis of transcription factors selectivity in responses to different xenobiotics.

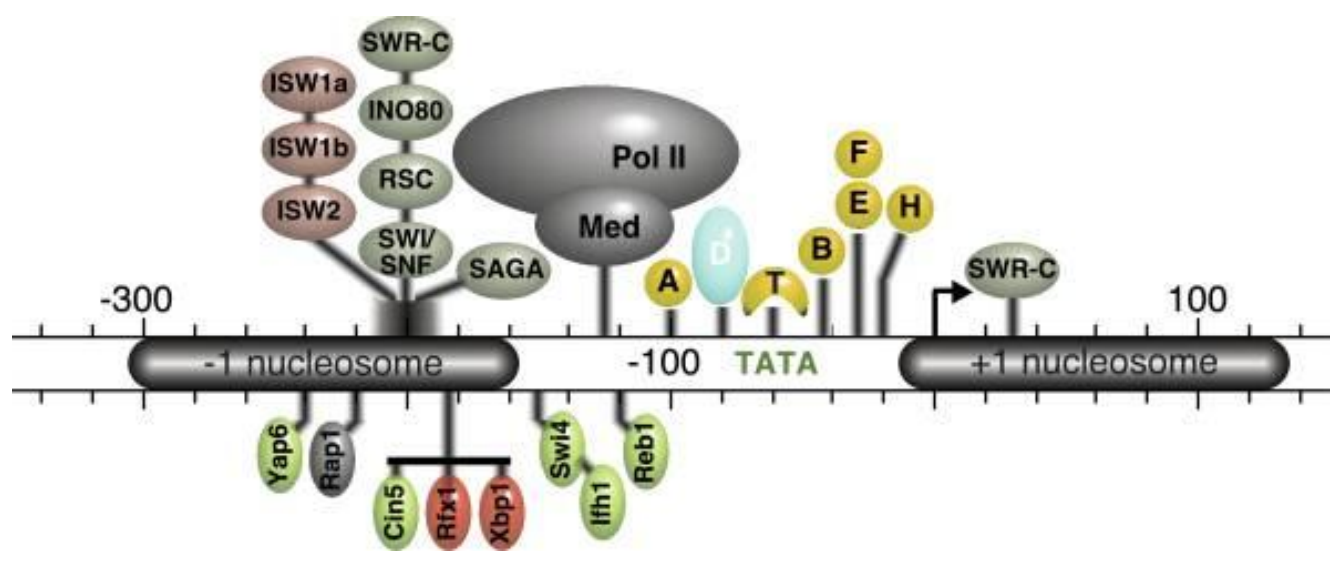

Figure 4-15: Spatial assembly of the transcription machinery

Spatial assembly of the transcription machinery and chromatin regulators has major effect on gene expression and transcriptional activity. Figure from (Venters and Pugh 2009) 


\section{Section V: Lipid pathway genes}

In describing the GO category groups emerging from the screens described here, it became apparent that the genes most dependent on Pdr1p/Pdr3p are involved in sphingolipid and phospholipid homeostasis. Besides structural functions sphingolipids and phospholipids are known to be involved in signalling (Sun, Taniguchi et al. 2000; Friant, Lombardi et al. 2001; Hannun and Obeid 2008). Another category is mitochondrial status which can be linked to stress response signalling (Manoli, Alesci et al. 2007). Mammalian cell lines which display a multidrug resistant phenotype show an increase in sphingolipid in the cell membrane and these cell lines show a change in ceremide regulation, a proapoptotic signal (Sietsma, Veldman et al. 2001). However, the link between sphingolipid/phospholipid signalling and PDR is less well understood in yeast. Liu and Sharom 1998 also showed that there is energy transfer between the ATPbinding domain of the $\mathrm{ABC}$-transporter and phosphatidylethanolamine derivatives which is sphingolipid ceremide-based using fluorescence resonance (Liu and Sharom 1998). Our results suggest that the link between sphingolipid and PDR , and genes like DPL1 which encode dihydrosphingosine phosphate lyase should be further investigated.

\section{Section VI: Genes predicted to be involved in the mediation/ modulation of the PDR response via network interaction}

It has been shown that genes which interact with one another in the network tend to work in the similar pathway or have similar function and can buffering each other mutation effects (Tong, Evangelista et al. 2001; Tong, Lesage et al. 2004). To investigate if genes that interact (physically and genetically) with our "hit" genes might be involved in modulating PDR phenotype, we performed xenobiotics spot dilutions and Western Blots. To maximise the chance of identifying genes that have major effect on the PDR phenotype, and not just downstream transporters, we only tested deletion of genes that interact with our enrichment genes (e.g. Chromatin remodelling complex, signalling genes), and with PDR1 PDR3. Most of these genes also have just missed the "cut off" of being identify as hits in the primary screen.

Out of the 18 gene deletions we tested 10 displayed either increases in xenobiotic sensitivity when deleted or reduced Pdr5p abundance in Western blot or both. 
These genes are ADA2, DEP1, ERG3, HEK2, HEX3, HSP82, LGE1, MNN10, NGG1, and SPT8. Six of these 10 genes showed xenobiotic hypersensitivity when deleted, and 4 of the 10 (with all but one overlap) caused a reduction in Pdr5p abundance when deleted (Table 4-4). Ngg1p has been shown to interact with the transcriptional activation domains of Pdr1p and Prd3p (Martens, Genereaux et al. 1996) and partially requires Ada2p. Nevertheless, this result comes from a prediction of PDR-functionality and strongly supports the role of transcription factors other than $P D R 1$ and PDR3, regulators, mediators in providing mechanisms for PDR response. These results also highlight chromatin modification as a specific response being not just a global pleiotropic transcriptional effect.

One gene that we found to be the odd one out was LGE1. Deletion of this gene in any background did not increase sensitivity towards any of the compounds tested but reduced Pdr5p abundance (Figure 4-10). LGE1 is an uncharacterised gene but may have a role in conveying a message between damaged mitochondrial and the nucleus in $\rho^{0}$ cells (Gulshan, Schmidt et al. 2008) and in histone H2B ubiquitination. Deletion of $L G E 1$ also causes a decrease in $P D R 3$ expression in $\rho^{0}$ cells. Importantly, LGE1 displays phenotypic suppression with PDR3. Overexpression of $P D R 3$ can bypass the requirement of $L G E 1$ for $P D R 5$ activation in $\rho^{0}$ cells (Zhang, Kolaczkowska et al. 2005). More importantly, LGE1 displays negative genetic interaction with GAL11, suggesting that Lge1p can compensate for the loss of GAL11, or provide an alternative pathway to achieve PDR phenotype. This might be the reason why GAL11 did not come up as positive hit in the primary screen. Together this evidence points towards an important role of Lge1p in the PDR response that might include the link between mitochondrial stress and activation of PDR by upregulation of PDR5 in a Pdr3p-dependent manner. We also show here the power of our genome-wide screen and interaction network in helping to ascertain the function of novel genes.

\section{Conclusion}

In conclusion, we used ABC-transporter proteins labelled with GFP as real-time reporters of the activation of Pdr1p and Pdr3p. The reporters were expressed in 
the S. cerevisiae genome deletion array genetic background (about 4500 strains), then treated with atorvastatin as a quintessential xenobiotic activator of PDR. We assayed for gene deletions that prevented normal up-regulation of Pdr5p-GFP and Yor1p-GFP in the presence of the drug by high-throughput quantitative confocal microscopy. From this primary screen, we observed that 54 genes deletions caused failure to upregulate our reporters. When we further tested these mutants in spot dilution assays 26 showed sensitivity towards the panel of 6 compounds tested. When we assessed the known functions of these 26 genes we found four different groups into which these genes fell. These are kinase/ Rho-GTPase signalling (BMH1, SNF4, SSZ1, BEM2, RHO4, GTR2, RPN10), mitochondria/phospholipid/sphingolipid and membrane integrity $(R C Y 1, C W H 43$, MDM39, PIB2, OPI3, PSD1, FMC1, YME1, DPL1), mediators (SRB8, ROX3), and chromatin modification/transcriptional activators/regulators (UME6, PDR1, PDR3, SWI4, KAP123, SPT7, GCN5, PIN4). Our results show that there must be other pathways, which can compensate for the loss of PDR1 and PDR3 and contribute to the PDR phenotype. For one, a signalling pathway and a context-specific chromatin remodelling must be involved, demonstrating an underappreciated role of signalling pathways in the PDR response (Figure 4-16). Presence of xenobiotics could possibly activate the kinase/GTPase MAPK stress response signalling. This signalling is then received by the SAGA/ chromatin remodelling complex as well as RNA polymerase II mediator complex. These complexes then recruit onto the PDR1 PDR3 promoters and switch on their expression. Pdr1p/Pdr3p can then activate many $A B C$-transporters and major facilitator genes. Xenobiotics are then pumped out of the cell. At the same time that this happens or when PDR1 and PDR3 are absent, the alternative pathways can be switched on as backups. Different stress-response transcription factors can be switched on in the presence of xenobiotics depending on the modifications to the SAGA complex or what subunits of RNA Pol II are recruited (represented by different colours in Figure 4-16). These TFs can then switch on the same target genes as Pdr1p/Pdr3p or the new sets of genes such as lipid, sterol, phospholipid/sphingolipid which can then change the membrane integrity and affect the xenobiotics uptake. Loss of mitochondrial genome $\left(\rho^{0}\right)$ can also activate the PDR response probably involving Lge1p (retrograde response). Other modulators in the PDR response also include members of the Hsp70 and Hsp90 protein families as well as ubiquitination 
proteins. Here, we have shown that it is very likely that there are upstream signalling pathway(s) to Pdr1p/Pdr3p, and that PDR responses can be achieved via compensatory pathway that are independent of Pdr1p/Pdr3p.

Many other open questions still remain about what may regulate Pdr1p/Pdr3p and contribute to PDR response. We found many proteins with unknown molecular function such as Cwh43p, a putative sensor/transporter containing several glycosylation and phosphorylation sites, which potentially can be used as regulatory sites during signal transduction. In the future we would like to generate hyperactive mutant form of PDR1 ( $p d r 1-3$ ) that increase Pdr5p expression, and see if any of the hit genes reported will reduce Pdr5p expression in this mutant background. In theory those genes should be up-stream of Pdr1p.

Our method can be adapted to many other cellular pathway systems to identify potential regulators, both activators and suppressors. This assay, done in live cells, can be used to investigate morphological changes due to mutations or chemicals per cells and quantify them. 


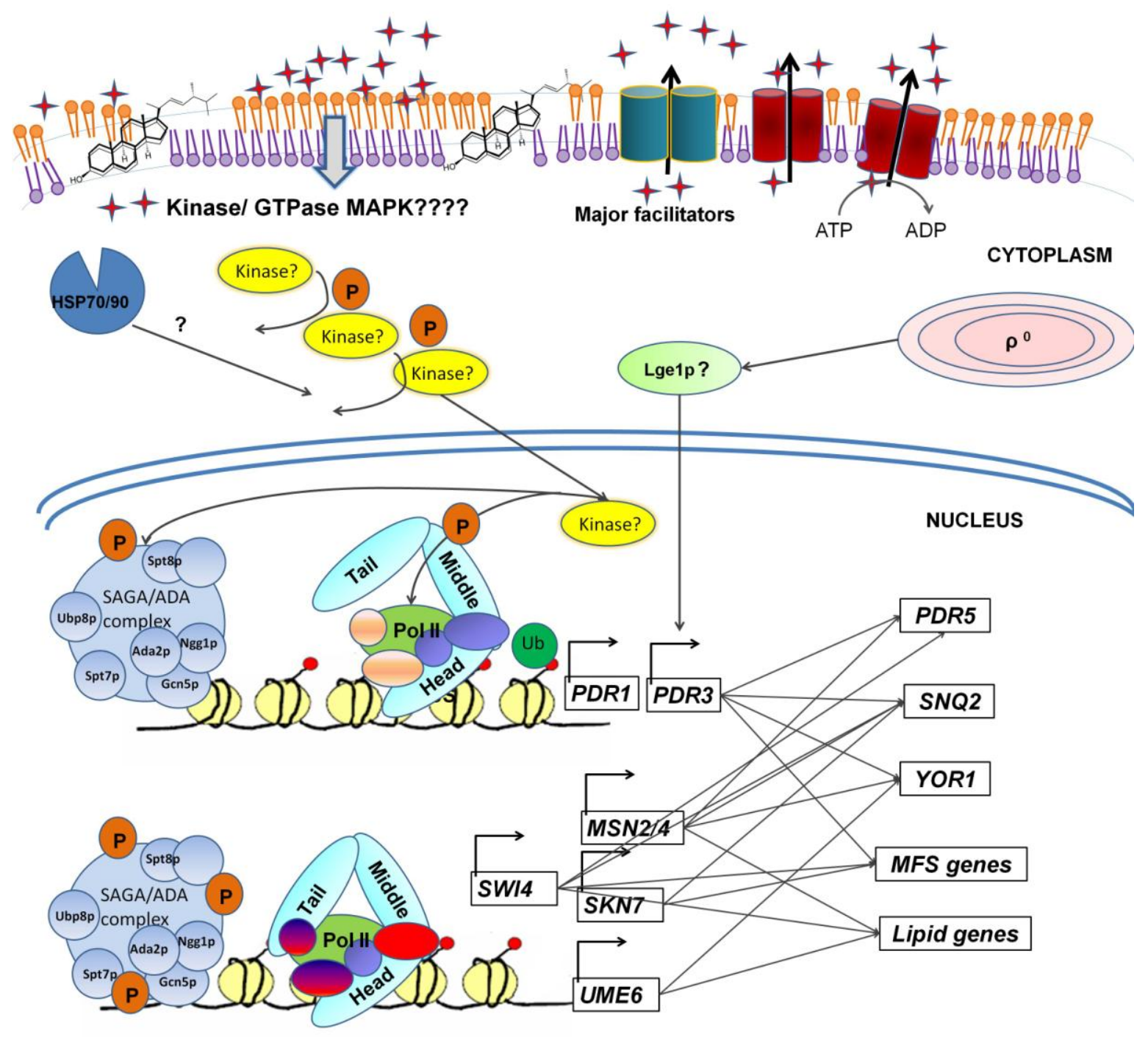

Figure 4-16: Possible alternative pathways to activate the PDR response in S. cerevisiae. 


\section{Chapter 5. Use of a Yap1p-GFP reporter to investigate relationships between oxidative stress and PDR}

\section{Introduction}

As described in the Introduction (Chapter 1), there is good reason to search for overlap of regulatory and other genes between stress pathways-such as the PDR pathways and the oxidative stress pathways. We investigated whether different stress inducing agents activated the same "sensors", or whether "sensors" are specific to different agents. As outlined, YAP1 is a key oxidative response gene to study from this standpoint.

Normally, oxidative stress response is assessed in three major ways. First, by directly measure cellular ROS such as nitric oxide. Second, by measure the resulting damage of oxidative stress to biomolecules, such as DNA damage or mitochondrial dysfunction (Halliwell and Whiteman 2004). Third, by detect the level of antioxidants such as Glutathione (GSH), the most abundant antioxidant in aerobic cells. However, ROS are hard to detect as they are highly reactive and short lived, thus making these methods problematic. Although mitochondrial dysfunction and DNA damage phenotypes provide a good oxidative stress indicator, they can be caused by many other stresses. Finally, acidification often used during protein purification, may also contribute to oxidation of GSH and other antioxidant enzymes, resulting in overestimation of the oxidized form of GSH. None of these methods, however will directly implicate genes involved in sensing, and regulating an oxidative stress response.

The aim of this chapter was to identify genes involved in Yap1p activation during oxidative stress induced by different oxidants using genome-wide, high content imaging. To do this, gene deletions were sought that prevent the normal (wild-type) nuclear re-localisation of a Yap1p-GFP reporter upon treatment with oxidants. Gene deletions that prevented the re-localisation of Yap1p-GFP from the cytoplasm to the nucleus in the presence of oxidative stress-inducing agents $\mathrm{H}_{2} \mathrm{O}_{2}$ and/or diethyl maleate were sought, as these are potentially involved in Yap1p regulation. The approach in this chapter, therefore, was to compare the results from the Yap1p-GFP screens with the results from the PDR screen to assess 
commonalities and overlaps between these two screens. It should be noted that we also initially tested Yap1p-GFP induction with diamide, but due to greater availability and a better phenotype that was obtained with diethyl maleate; we chose to use diethyl maleate for the further studies.

As with the PDR studies, searching for these genes using a genome-wide approach means we can explore all the non-essential genes which might buffer pathways related to Yap1p. In addition, using Yap1p-GFP as reporter meant we can directly observe the real-time transcriptional activation of the oxidative stress response, and explore upstream signalling genes that are regulating the process. This chapter describes the first genome-wide, high-content microscopy study of possible regulators of the oxidative stress response in S. cerevisiae, and how they might link Yap1p and the PDR response.

\section{Materials and Methods}

\section{Compounds and Concentration used}

Hydrogen peroxide, diamide and diethyl maleate were all purchased from Sigma Aldrich.

Hydrogen peroxide was diluted to $10 \mathrm{mM}$ stock in sterile distilled water on the day of use. The final concentration in the media was $1 \mathrm{mM}$. Diamide and diethyl maleate stock was made on the day of use in sterile distilled water, with the final concentration in the media of $1.5 \mathrm{mM}$ and $2 \mathrm{mM}$ respectively. Cells were incubated in 384 well PekinElmer Cell carrier plates with and without drugs at $30^{\circ} \mathrm{C}$ for 90 $\min$.

\section{Yap1p GFP Reporter Strain/ Screens}

Commercial YAP1 with the C-terminal GFP fluorescent tag from Invitrongen was used. This strain was mated against YCG253 (Table 2-2 page 66) and sporulated for 7 days at $20^{\circ} \mathrm{C}$. Tetrad dissection and haploid selection was conducted according to General Materials and Methods (page 67). The resultant strain was YCG379 (Table 2-2 page 66). Cytoplasmic RFP mCherry and RFP with NLS were used as internal controls.

To investigate the modulators controlling Yap1p transcriptional activity, YCG379 was mated against the deletion mutant array as described in the PDR screen 
according to the General Materials and Methods page 67. After final haploid selection, the cells carrying the deletion and the Yap1p-GFP reporter were then subjected to $1 \mathrm{mM}$ hydrogen peroxide, or $2 \mathrm{mM}$ diethyl maleate and incubated for $90 \mathrm{~min}$ at $30^{\circ} \mathrm{C}$ before imaging in the PekinElmer Opera ${ }^{\mathrm{R}}$ High content microscope with the settings as described (General Material and Methods page 71)

\section{Data analysis}

As with the PDR screen, images were analysed using Acapella software (script modified by Peter Bircham). For the peroxide screen (in which nuclear localisation was very clearly observed in the treated experiments), the ratio between $\frac{\text { NuclearGFP }}{\text { Nucleated }_{\text {rearGFP }} \text { control }}$ was calculated. A ratio of 1 means that there was no difference between the $\mathrm{H}_{2} \mathrm{O}_{2}$ treated cells and the control. Deletion of HIS3 (Dhis3) is a control commonly used in gene deletion screens as it has no effect on the process of interest. The deletion set contains a border of $\Delta h i s 3$ colonies on each plate as a control. The ratios of nuclear GFP between control and treated cells (equation above) of all $\Delta$ his 3 colonies were averaged and the standard deviation was calculated. Gene deletions were considered "hits" if the nuclear GFP ratio was less than 2 standard deviations from the mean ratio of the average control $\Delta$ his3 (That is, deletion of the gene prevented normal "wild-type" nuclear localisation of Yap1p following $\mathrm{H}_{2} \mathrm{O}_{2}$-induced-oxidative stress response). If there was no nuclear localisation of Yap1p-GFP in treated cells, it is suggested that the deletion these cells are carrying is a modulator of the transcriptional activity of Yap1p.

For the diethyl maleate screen, Texture Analysis using the Acapella Software was used for analysis, as treatments resulted in the nuclear localisation of Yap1p-GFP as well as punctate staining in the cytoplasm (Texture analysis page 71 ). The ratio of $\frac{\text { NuclearGFP }}{\text { CytoplasmGFP }}$ the treated and control samples for each gene deletion was calculated. The Z-score of the difference between the ratio of treated and control was then calculated (Z-score formula was given in the General Material and Methods page 71). A Z-score of zero mean there is no difference between cells treated with diethyl maleate and the untreated control cells. Gene deletions resulting in Z-scores below 4.5 were considered as "hits" and correspond to genes 
which modulate the transcriptional activity of Yap1p in the diethyl maleate-induced oxidative stress response.

\section{Yap1p-GFP pulse chase experiment}

Cells containing the Yap1p-GFP reporter were subjected to $1 \mathrm{mM} \mathrm{H}_{2} \mathrm{O}_{2}$ for 10 minutes then $100 \mu \mathrm{g} / \mathrm{ml}$ cycloheximide was added to stop protein synthesis of new Yap1p-GFP i.e. the protein synthesis effects of the oxidation were "chased". Under chase conditions, cell images were taken every 15 min for $6 \mathrm{~h}$ using the automated PerkinElmer Opera® High Content confocal microscope. These images were analysed using Acapella software to measure GFP intensity in the cytoplasm and the nucleus. 


\section{Results}

\section{Yap1p-GFP reporter behaviour in the presence of oxidants}

We found that Yap1p with C-terminal GFP reporter localised to the cytoplasm in non-treated cells and localised to the nucleus upon $\mathrm{H}_{2} \mathrm{O}_{2}$ treatment (Figure 5-1). Therefore, it may be concluded that the GFP-tag at the Yap1p C-terminus does not interfere with the normal localisation of the transcription factor. The nuclear localisation was specific to the oxidative stress response since Yap1p-GFP treated with atorvastatin (PDR-stress) did not result in nuclear localisation.

In diamide and diethyl maleate treatments, (both of which are free thiol oxidizing agents) the Yap1p-GFP reporter localised to the nucleus with some punctate spots in the cytoplasm. Diethyl maleate resulted in more complete Yap1p nuclear localisation than diamide (Figure 5-2). Due to both the availability and diethyl maleate resulted in more complete nuclear localisation of the Yap1p-GFP reporter, diethyl maleate, and not diamide, was used for screening in this dissertation. 


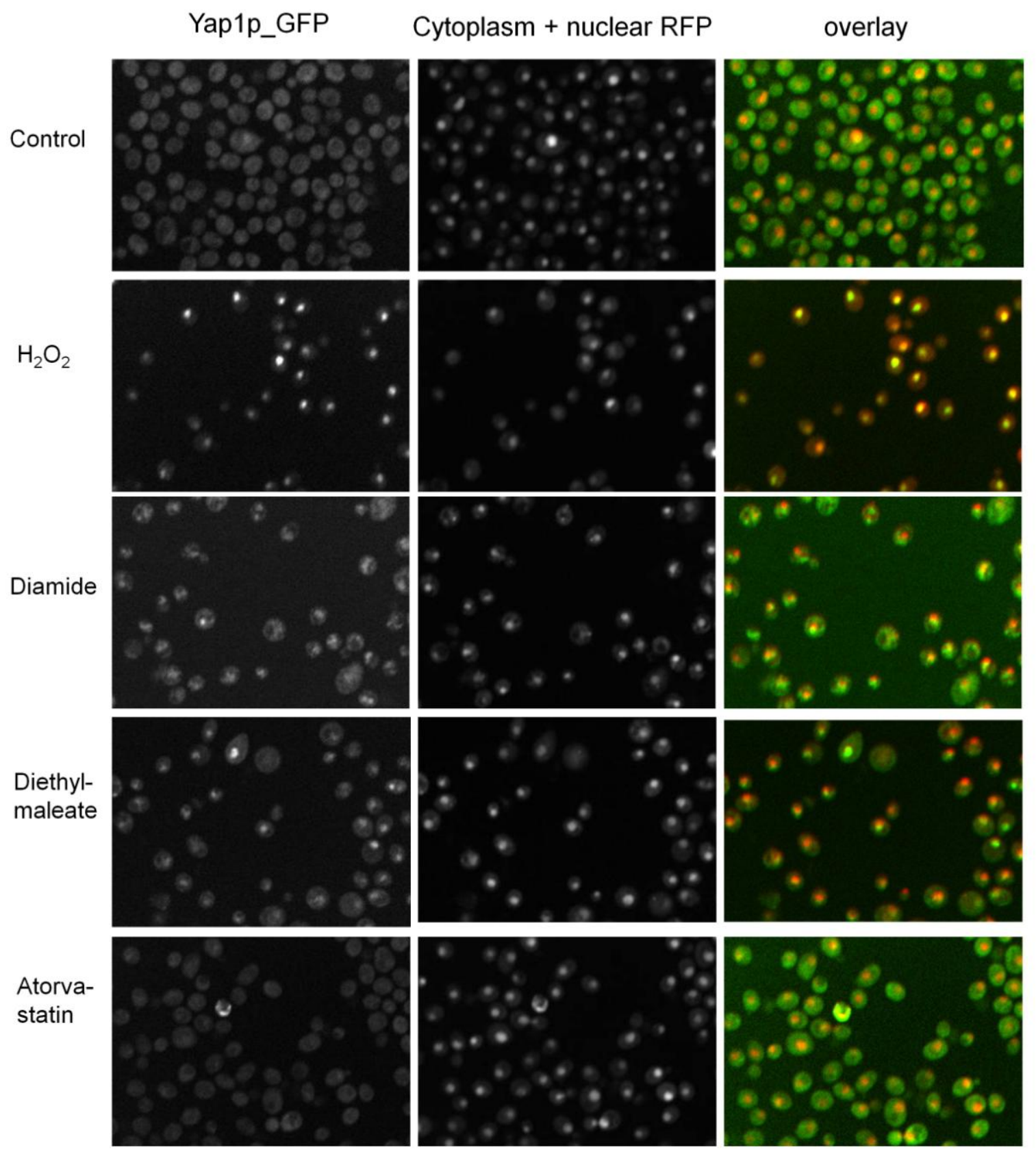

Figure 5-1: Yap1p-GFP reporter treated with different oxidants.

Yap1p-GFP with no oxidant treatment localised to cytoplasm. With oxidants, Yap1p-GFP localised to nucleus. The reporter contains cytoplasmic mCherry and a RedStar2-nuclear localisation signal (NLS) as an internal control. Atorvastatin (35 $\mu \mathrm{M})$ was used asnegative control. 


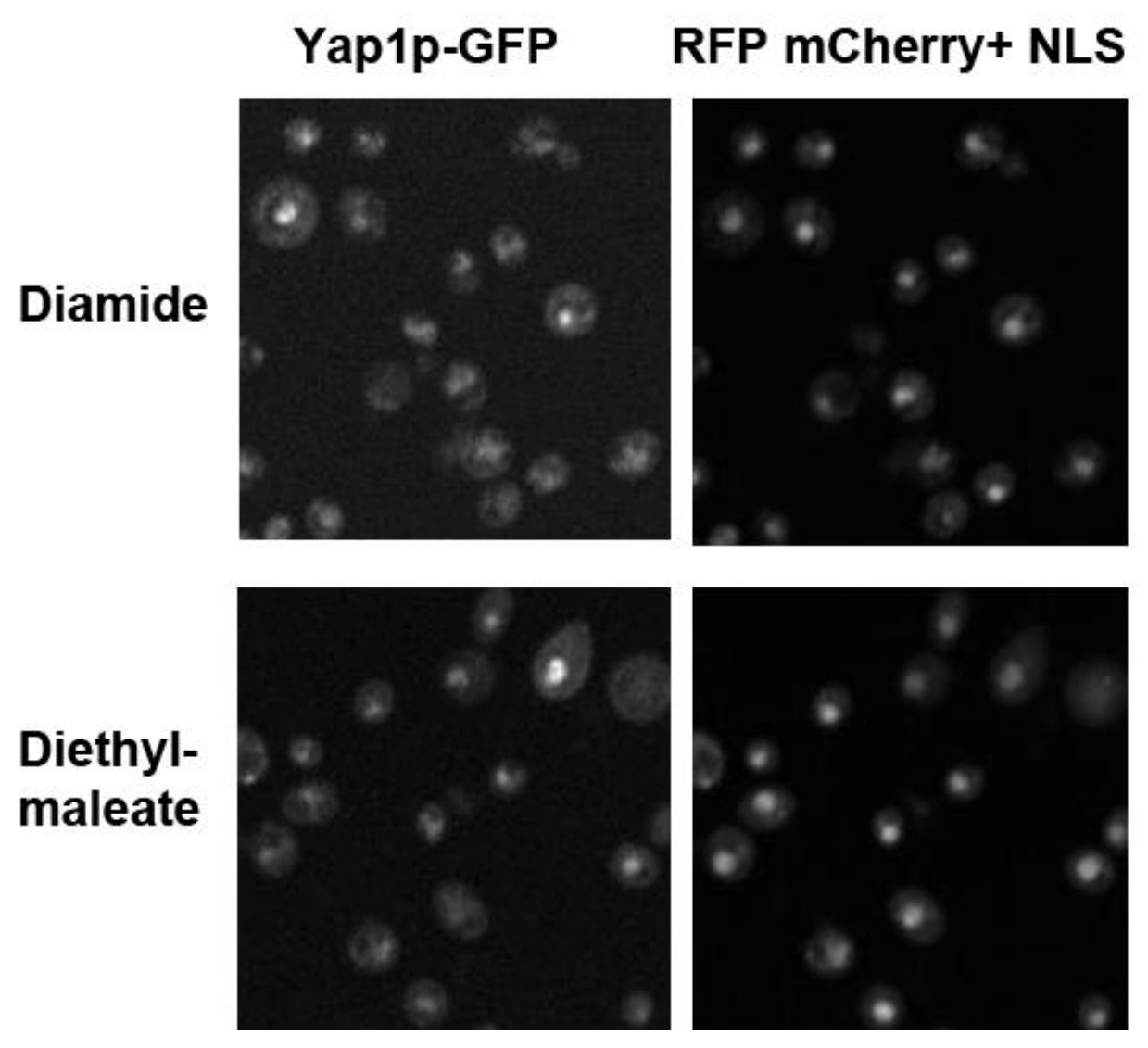

Figure 5-2: The punctate pattern of Yap1p-GFP observed when cells were treated with diamide or diethyl maleate.

\section{Yap1p -GFP reporter peroxide $\left(\mathrm{H}_{2} \mathrm{O}_{2}\right)$ and diethyl maleate screens}

In order to identify possible regulators/modulators of Yap1p in the oxidative stress response, we screened the $S$. cerevisiae deletion set for deletions that prevent the normal Yap1p-GFP nuclear localisation upon treatment with oxidants.

Example of gene deletions which resulted in cytoplasmic retention of Yap1p upon peroxide treatment i.e. "hits" are shown in Figure 5-3 and Table A-2 (Appendix I), and for diethylmaleate in Figure 5-4 and Table A-3 (Appendix I). The quantitative changes caused by the deletions shown in these figures are seen by comparing the gene deletion images with the wild-type control (Panel (a) in each figure). Corresponding panels show nuclear localisation distinguished by the red fH2Ouorescent dye mCherry attached to the nuclear localisation signal (NLS). Each oxidant screen was repeated twice and the results were reproducible. There were 56 genes recorded as "hits" in the $\mathrm{H}_{2} \mathrm{O}_{2}$ treatment and 51 in the diethyl maleate treatment. There was no significant overlap between genes identified in the two oxidants screens. Hits from both screens were enriched in genes involved 
in chromosome/DNA integrity, transcriptional activator/modulation, and genes that respond to environmental stresses as showed in Figure 4-5, Figure 4-6, Table 4-1, and Table 4-2.

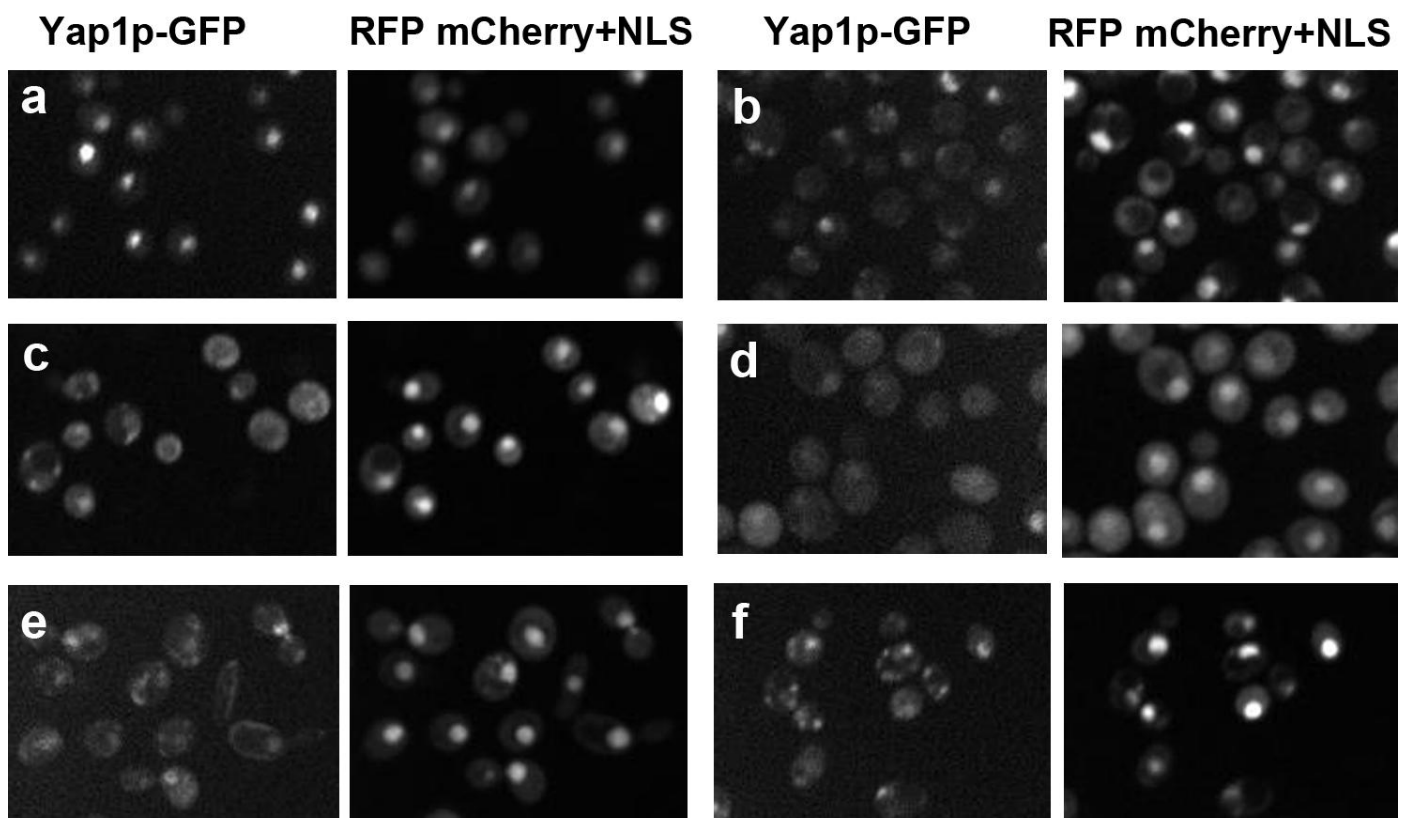

Figure 5-3 : Examples of gene deletions that prevented normal nuclear localisation of Yap1p-GFP upon treatment with $1 \mathrm{mM} \mathrm{H}_{2} \mathrm{O}_{2}$.

a) wt Yap1p-GFP, b) $\Delta a d a 2$, c) $\Delta g p x 3$ d) $\Delta k a p 123$, e) $\Delta m m s 1$, f) $\Delta p d e 2$. The reporter contains cycloplasmic mCherry and NLS as internal controls.

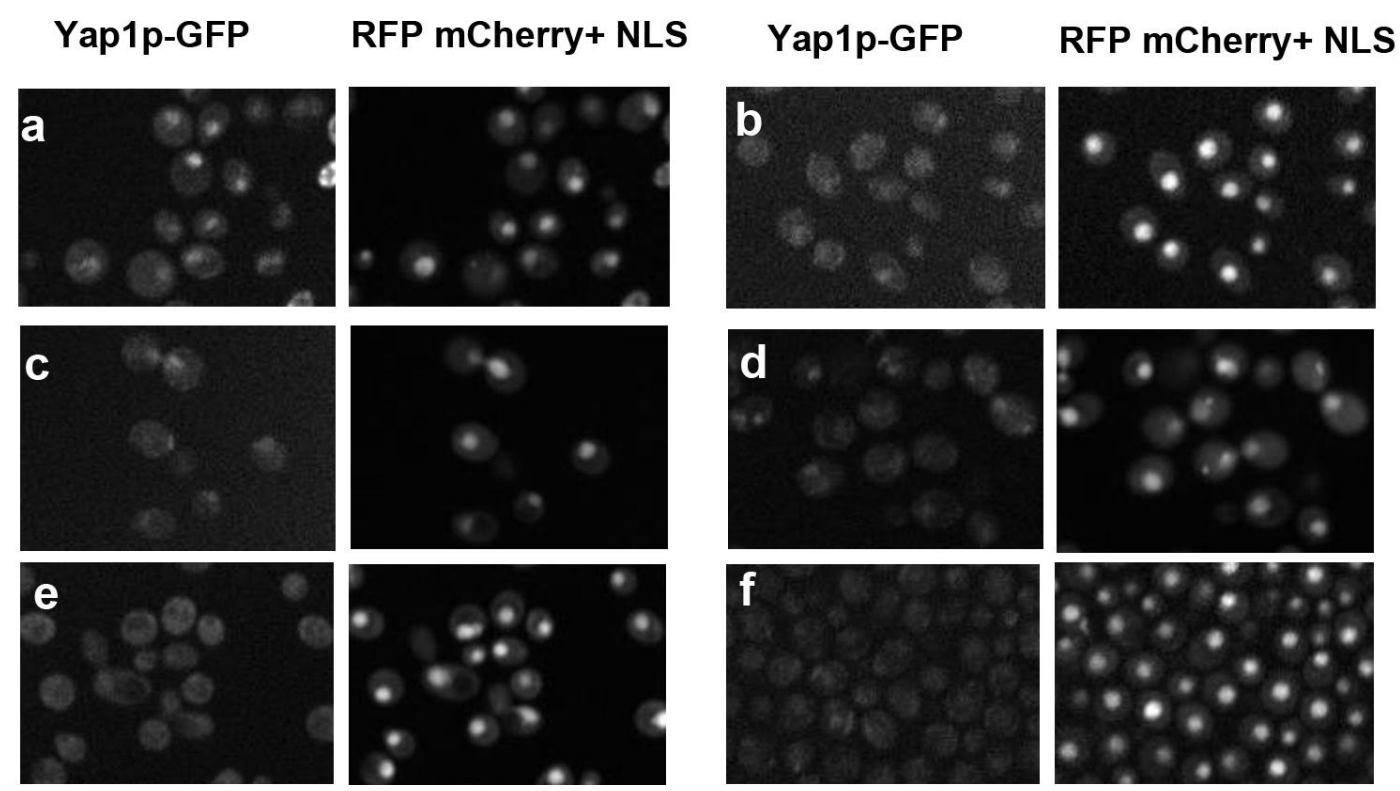

Figure 5-4 : Examples of gene deletions that prevented normal nuclear localisation of Yap1p-GFP upon treatment with $2 \mathrm{mM}$ diethyl maleate.

a) wt Yap1p-GFP, b) $\Delta g p x 2$, c) $\Delta m m s 1$ d) $\Delta r a d 54$, e) $\Delta t s a 1$, f) $\Delta y b p 1$. The reporter contains cycloplasmic mCherry and NLS as internal controls. 


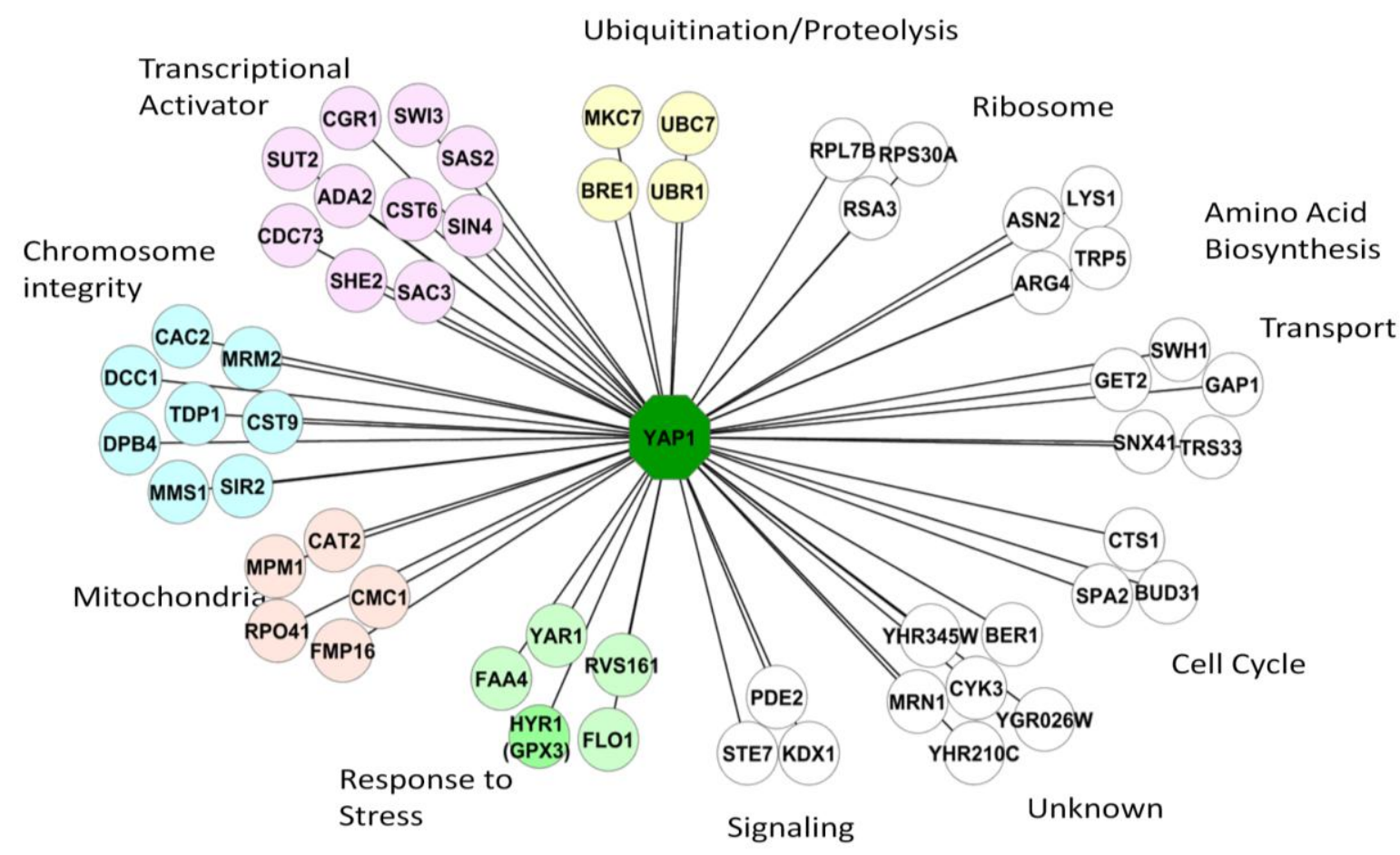

Figure 5-5 : Gene deletions that prevent normal nuclear localisation of Yap1p-GFP upon $\mathrm{H}_{2} \mathrm{O}_{2}$ treatment.

Each connection represents a possible regulatory relationship between YAP1 and the associated gene. Darker green node represents known regulator of Yap1p.

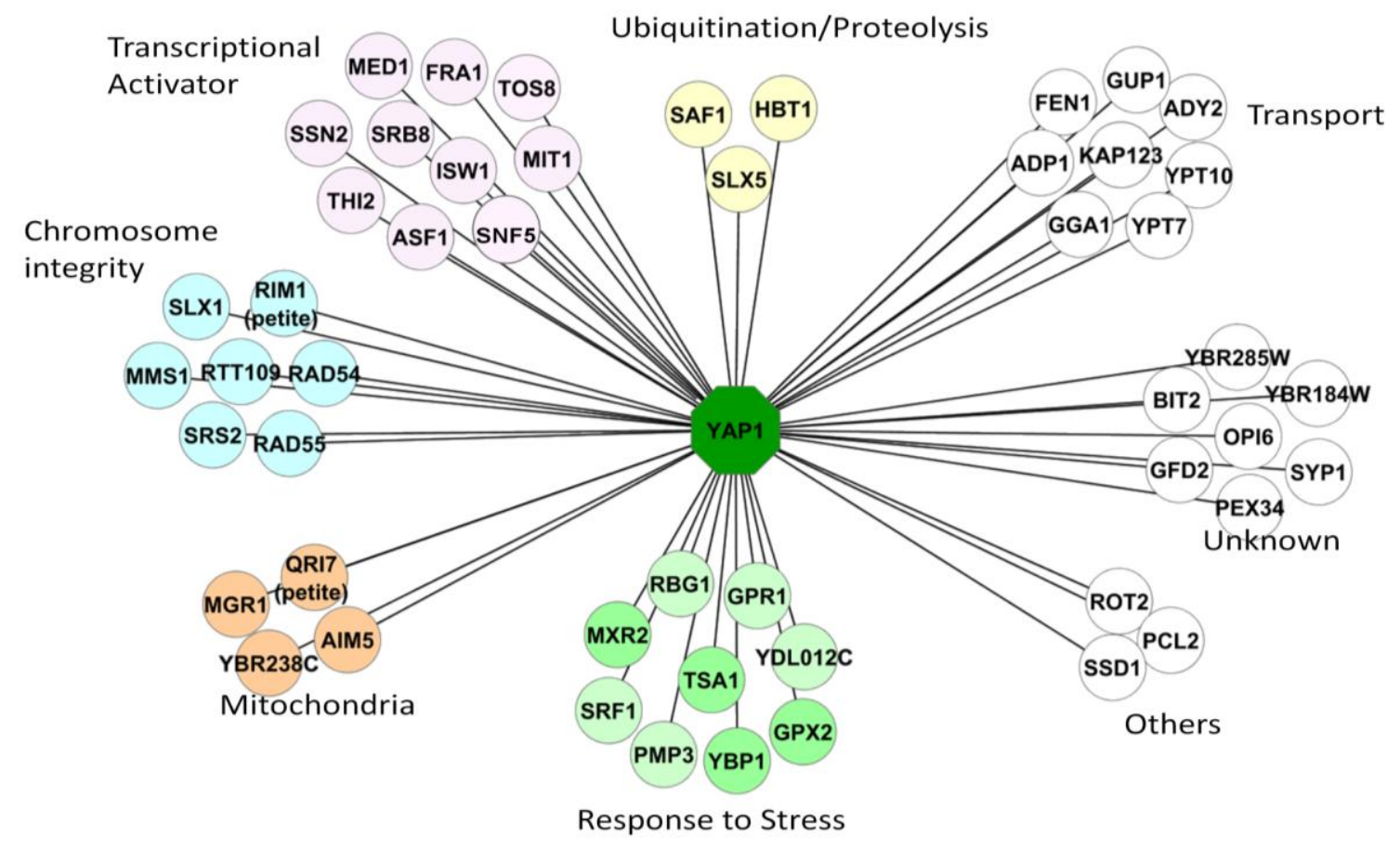

Figure 5-6 : Gene deletions that prevent normal nuclear localisation of Yap1p-GFP upon diethyl maleate treatment.

Annotations are as described in Figure 4-5. 
Table 5-1 :GO function enrichment of gene deletions preventing normal nuclear localisation of Yap1p-GFP reporter upon $\mathrm{H}_{2} \mathrm{O}_{2}$ treatment

\begin{tabular}{|c|c|c|}
\hline $\begin{array}{l}\text { GO term } \\
\text { histone acetyltransferase activity }\end{array}$ & $\begin{array}{l}\text { p-values } \\
0.01693\end{array}$ & $\begin{array}{l}\text { Genes/ORF } \\
\text { ADA2/YDR448W } \\
\text { SAS2/YMR127C }\end{array}$ \\
\hline $\mathrm{N}$-acetyltransferase activity & 0.04278 & $\begin{array}{l}\text { ADA2/YDR448W } \\
\text { SAS2/YMR127C }\end{array}$ \\
\hline ubiquitin-protein ligase activity & 0.02439 & $\begin{array}{l}\text { BRE1/YDL074C } \\
\text { UBR1/YGR184C } \\
\text { CST9/YLR394W } \\
\text { UBC7/YMR022W }\end{array}$ \\
\hline DNA replication & 0.0381 & $\begin{array}{l}\text { DCC1/YCL016C } \\
\text { DPB4/YDR121W } \\
\text { MMS1/YPR164W } \\
\text { SIR2/YDL042C }\end{array}$ \\
\hline phosphoric diester hydrolase activity & 0.04813 & $\begin{array}{l}\text { TDP1/YBR223C } \\
\text { PDE2/YOR360C }\end{array}$ \\
\hline
\end{tabular}

Table 5-2 :GO function enrichment of gene deletions preventing normal nuclear localisation of Yap1p-GFP reporter upon diethyl maleate treatment

\begin{tabular}{lll}
\hline GO term & p-values & Genes/ORF \\
\hline RNA polymerase II transcription factor & 0.0026 & THI2/YBR240C \\
binding transcription factor activity involved & & SRB8/YCR081W \\
in positive regulation of transcription & & SSN2/YDR443C \\
& & MMS1/YPR164W \\
response to DNA damage stimulus/DNA 0.0069 & RAD54/YGL163C \\
repair & & RAD55/YDR076W \\
& RTT109/YLL002W \\
& SLX1/YBR228W \\
& SLX5/YDL013W \\
& SRS2/YJL092W \\
& TSA1/YML028W \\
cellular response to oxidative stress & & GPX2/YBR244W \\
& & MXR2/YCL033C \\
& 0.0511 & TSA1/YML028W \\
& & YBP1/YBR216C \\
\hline
\end{tabular}

Note: Some of the gene deletions show GO enrichment in stress response but with p-value more than 0.1 (for example GPX3 from $\mathrm{H}_{2} \mathrm{O}_{2}$ screen) 


\section{Pulse chase experiments of Yap1p-GFP Reporter}

From the $\mathrm{H}_{2} \mathrm{O}_{2}$ screen, we identified $\mathrm{GO}$ enrichment of genes involved in ubiquitination and proteolysis. To further test if the ubiqutination process is involved in Yap1p regulation, we conducted pulse chase experiments as described. Cells labeled with Yap1p-GFP in the cytoplasm were "pulsed" with $\mathrm{H}_{2} \mathrm{O}_{2}$ for 10 minutes and then "chased" in the presence of cycloheximide - inhibiting further protein synthesis under these conditions. Thus, all the changes in GFP localisation should be correspond to re-localisation of pre-existing Yap1p-GFP.

If Yap1p transcriptional activity is only controlled by subcellular localisation (cytoplasm and nucleus), we should see a decline of Yap1p-GFP intensity inside the nucleus and increase of Yap1p-GFP intensity in the cytoplasm over time as cells adapt to the oxidative stress. However, if proteolysis is involved either in the nucleus or in cytoplasm, we should see Yap1p-GFP intensity decline in these subcellular locations over time.

We found that cytoplasmic Yap1p-GFP stayed constant throughout the experiment except in SC (media) controls where cycloheximide was not added. Nuclear Yap1p-GFP intensity started to decline 105 minutes after cycloheximide was added. The overall Yap1p-GFP (Nucleus+cytoplasm) in the experiment with cycloheximide treatment decreased slightly but not significantly over time (Figure 5-7) 


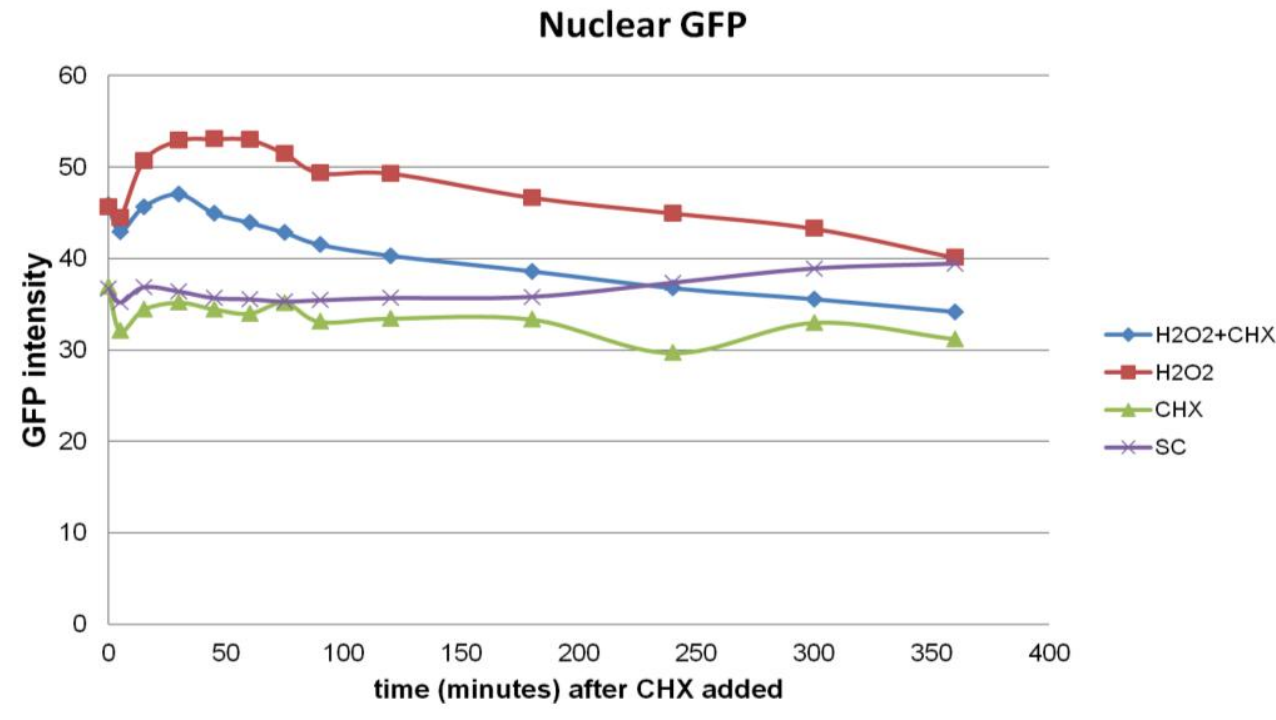

Cytoplasm GFP

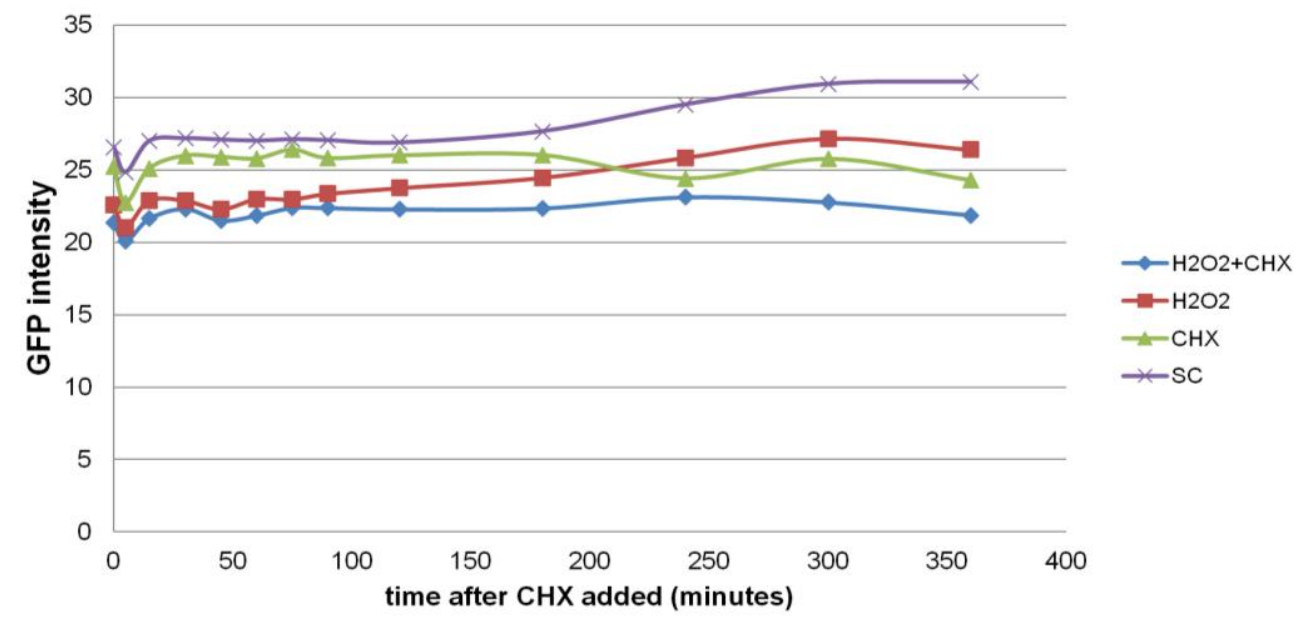

Total Yap1_GFP protein (nucleus+cytoplasm)

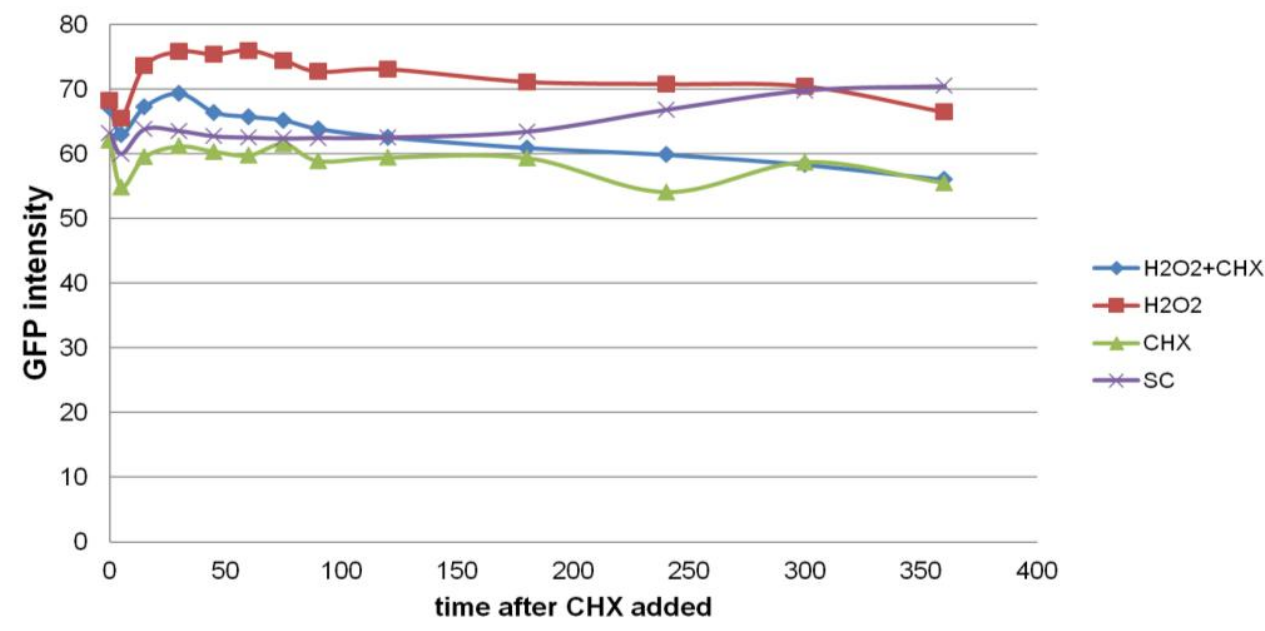

Figure 5-7 : Pulse chase experiment of Yap1p-GFP treated with $\mathrm{H}_{2} \mathrm{O}_{2}$ 


\section{Discussion}

\section{Section I: The validity of the reporter}

The Yap1p-GFP localised to the nucleus in an oxidative stress specific fashion. In screens for both oxidants, some of the known regulators of Yap1p nuclear localisation appeared as "hits", indicating that the screen is functioning correctly. The genes were GPX3 in the $\mathrm{H}_{2} \mathrm{O}_{2}$ screen and YBP1 in diethyl maleate screen, proving that our reporter was valid.

An interesting result we found was the punctate pattern and partial nuclear localisation of Yap1p-GFP in diethyl maleate and diamide-treated cells (Figure 5-1) not previously described in the literature. An explanation for this might be that the commercial Yap1p reporter in this study has GFP linked to the C-terminus of the proteins, whilst in most published studies GFP was linked to the N-terminus. The C-terminal of Yap1p is known to contain a cysteine-rich domain and NLS, which controls nuclear localisation of the protein upon encountering oxidative stress. Disulfide bonds between cysteine residues in the $\mathrm{C}$-terminus are required for a normal response to diamide (Kuge, Arita et al. 2001). Thus, GFP linked to the Cterminus might have influenced the formation of disulfide bonds in the $\mathrm{C}$-terminus during diamide and diethyl maleate response, resulting in partial nuclear localisation and the punctate pattern of Yap1p.

It is noteworthy that $\mathrm{H}_{2} \mathrm{O}_{2}$ causes oxidative stress by generating ROS while both diamide and diethyl maleate are weaker oxidizers and cause oxidative stress by depleting the antioxidant GSH, (Bauman, McKim Jr et al. 1992). Nevertheless, the mechanisms of actions for these two thiol-oxidant compounds are different. Diamide causes disulfide formation between itself and GSH, resulting in GS-SH, which then can modify other proteins (C-GS-SH). On the other hand diethyl maleate chemically modifies GSH. This might be another explanation for why different phenotypes, with regard to Yap1p nuclear localisation, were observed when cells were treated with different oxidants.

The C-terminal disruption might also be the reason why we did not observe the expected lack of nuclear localisation of Yap1p-GFP in $\Delta y b p 1$ cells in the $\mathrm{H}_{2} \mathrm{O}_{2}$ treatment. It may be that the C-terminus GFP has an effect on the minor conformational change during the interaction between Ybp1p and Yap1p in the 
presence of $\mathrm{H}_{2} \mathrm{O}_{2}$. This effect seemed to be more specific to $\mathrm{H}_{2} \mathrm{O}_{2}$, where disulfide bond formation between $\mathrm{N}$ - and $\mathrm{C}$ - terminus are needed for Yap1p nuclear localisation(Delaunay, Pflieger et al. 2002). While in diethyl maleate, no disulfide bridge between these two terminals is needed and minor disruption causing by $\mathrm{C}$ terminal GFP tagged is more forgiving. We think C-terminal GFP-Yap1p can still be used as a valid oxidative stress reporter because Yap1p-GFP can still respond to oxidants as $\triangle y b p 1$ still prevented Yap1p-GFP nuclear localisation in diethyl maleate, and $\Delta g p \times 3$ still came up as hit in $\mathrm{H}_{2} \mathrm{O}_{2}$ screen as expected. As mentioned in the introduction, the position of Yap1p disulfide bonds formed during oxidative stress is oxidant dependent. Furthermore, the correct conformation is essential for nuclear localisation of Yap1p (Coleman, Epping et al. 1999; Kuge, Arita et al. 2001; Gulshan, Rovinsky et al. 2005). However, the role of the Cterminus and $\mathrm{N}$-terminus cysteine rich in Yap1p regulation are still not entirely clear.

We found no significant overlap in hits from these two oxidants screens $(\Delta m m s 1$ was the only overlap), leading us to believe that cells use different sets of genes to regulate Yap1p transcriptional activity in the presence of different oxidants. Nevertheless, the general functions of the genes involved in Yap1p regulation are similar between the two oxidants screens, with genes functioning mainly in DNA repair and genome integrity (mitochrondrial), and mediator and stress responses (Table 5-1and Table 5-2). These will be discussed below.

\section{Section II. Yap1p regulation is part of the DNA repair and DNA integrity network.}

Many of the hit genes from both oxidant screens were involved in chromosome integrity or DNA repair and showed GO enrichment for these processes (Table 5-1 and Table 5-2). This might be due to the fact that ROS, which cause oxidative stress are a major cause of DNA damage. Pan et al. (2006) showed that the DNA integrity network consists of several principal modules involved in DNA replication, DNA-replication checkpoints, and the oxidative stress response. All three major modules act as guardians against lethal spontaneous DNA damage (Pan, Ye et al. 2006). The hits identified in this study- CAC2, DCC1, DPB4, TDP1, SIR2, MMS1, RTT109, SLX1, SLX5, SRS2, RAD54, RAD55, and TSA1 all have been shown to involved in such DNA damage pathways and DNA integrity networks (Pan, Ye et 
al. 2006; Workman, Mak et al. 2006). Therefore, regulation of Yap1p is linked with the chromosome integrity network.

In addition, Kuge et al. (1997) showed that Yap1p DNA binding capacity was moderately increased in the response to DNA damage. They suggested that this might involve post-translational modification within the DNA-binding domain in the $\mathrm{N}$-terminus of Yap1p (Kuge, Jones et al. 1997). Rowe et al. (2012) also suggested that DNA damage might activate transcriptional activity of Yap1p (Rowe, Degtyareva et al. 2012). The importance of the DNA integrity network as part of Yap1p regulation was also emphasized by the fact that our only overlap in the two different oxidants screens was MMS1. Mms1p is a subunit of an E3 ubiquitin ligase complex involved in replication repair (Hryciw, Tang et al. 2002). Additionally, Tsa1p which is a known Yap1p target and oxidative stress modulator (Inoue, Matsuda et al. 1999), has been shown to involved in the DNA damage stress response and DNA repair and might be involved in a negative feedback loop controlling ROS, cellular redox state and Yap1p (Pan, Ye et al. 2006).

\section{Section III: A different transcriptional activation complex and different subunits of RNA polymerase II mediator complex are used for Yap1p regulation when exposed to different oxidants}

GO term analysis showed significant enrichment in genes involved in chromatin remodelling and the RNA polymerase II mediator complex in both $\mathrm{H}_{2} \mathrm{O}_{2}$ and diethyl maleate screens. Though the genes mostly differ, this is a similar functional enrichment seen with the PDR response. Two genes that overlap in both sets of responses are SRB8 and KAP123. In the $\mathrm{H}_{2} \mathrm{O}_{2}$ screen, most of the genes identified as hits are involved in transcriptional activation and chromatin remodelling such as $A D A 2$ and $S W I 3$, and SAS2 and one subunit of RNA polymerase II mediator, SIN4. On the other hand, more RNA polymerase II mediator subunits were identified in the diethyl maleate screen, (SSN2, SRB8, and MED1) along with the chromatin remodelling complexes (SNF5, ISW1, ASF1) and transcription factors (TOS8, MIT1, THI2, and FRA1).

The SWI/SNF nucleosome-remodelling complex is known to activate genes in the stress response and might be controlling the oxidative stress response in the MAP kinase pathway (Ikner and Shiozaki 2005). As mentioned in the introduction, ADA/ 
SAGA complexes have been shown to control transcription of many stress response genes, including $P D R 1$ in the multidrug resistance response. Interestingly, $R O X 3$, which had been shown to result in $\mathrm{H}_{2} \mathrm{O}_{2}$ sensitivity when deleted (Gulshan, Rovinsky et al. 2005), was not identified as a hit in our screens, but SIN4 which is another mediator in the same complex was. Our explanation is that other mediator subunits like Sin $4 p$ and Ssn2p might be more specific to the oxidative stress response as $\operatorname{Sin} 4 p$ has been shown to physically interact with Yap1p (Gavin, Aloy et al. 2006). Another explanation is that other transcription factors or chromatin remodelling complexes can provide the compensatory pathway which can bypass the requirement of Rox3p.

The fact that we identified a different set of genes with similar functions when cells were challenged with different oxidants suggested to us that different subsets of chromatin remodeling and mediator complexes are used for different oxidants, and hence result in a different subset of Yap1p targets being expressed.

\section{Section IV: Genes involved in ubiquitination might regulate Yap1p and are more $\mathrm{H}_{2} \mathrm{O}_{2}$ specific}

We also observed that our screens identified many of the genes involved in the ubiquitination process. This observation was more pronounced in the $\mathrm{H}_{2} \mathrm{O}_{2}$ screen than in the diethyl maleate screen. These genes were BRE1 (E3 ubiquitin ligase), UBR1 (E3 ubiquitin ligase), and UBC7 (ubiquitin conjugating enzyme). The enrichment of ubiquitination genes was also confirmed by GO enrichment analysis (5\% level of significance).

Ubiquitination is used for protein regulation by either tagging them for degradation, or modifying them to perform certain functions, such as removing the suppressor proteins from active protein complexes (Ciechanover 1994; Ciechanover and Iwai 2004). One of the hits identified in the $\mathrm{H}_{2} \mathrm{O}_{2}$ screen, Ubr1p has been shown to control the oxidative stress response via degradation of active Pap1p, a homologue of Yap1p in S. pombe (Kitamura, Taki et al. 2011). Constitutively active Pap1p in the nucleus can result in growth inhibition in a ubr1 mutant (Kitamura, Taki et al. 2011). Ubr1p has also been found to regulate nitric oxide sensing in mammalian cells (Hu, Sheng et al. 2005). In mammals, b-ZIP transcription factors 
functioning in oxidative stress are regulated via ubiquitination proteolysis in the cytoplasm (Kitamura, Taki et al. 2011).

Our results are consistent with the literature and strongly suggest that Yap1p is regulated by Ubr1p, and possibly by other ubiquitination-related proteins. This result was also supported by the pulse chase experiment, where we observed the decline in Yap1p-GFP intensity over time in the nucleus, but no change in cytoplasm. Since cytoplasmic Yap1p-GFP did not significantly change over time, we speculated that Yap1p-GFP in the nucleus might be degraded, presumably after all the oxidative stress response genes had already been activated. This degradation process might involve Yap1p being ubiquitinated before entering the nucleus. Nonetheless, ubiquitination seems to give cells another way to regulate Yap1p in addition to nuclear transport.

Our results suggest ubiquitination (involved in protein degradation) plays a role in Yap1p regulation and is consistent with Gulshan et al. 2012, whose group recently showed that Yap1 degradation is accelerated in response to oxidative stress induced by both $\mathrm{H}_{2} \mathrm{O}_{2}$ and diethyl maleate (Gulshan, Thommandru et al. 2012). Not consistent with their results however, we could see no GO enrichment for ubiquitination genes in the diethyl maleate screen. Gulshan et al 2012 looked at degradation after Yap1p had localised to the nucleus and it could be that nuclear degradation of Yap1p is a common response toward all oxidants. On the other hand, ubiquitination and controlling of Yap1p nuclear localisation is more specific to Yap1p regulation in the presence of $\mathrm{H}_{2} \mathrm{O}_{2}$. In the future, to test this hypothesis, a pulse chase and Yap1p-GFP microscopy experiments using other oxidants or conducted in different proteolysis-related mutation backgrounds would be useful.

\section{Section V: Stress response genes which are targets of Yap1p might provide a feedback loop for Yap1p regulation}

Some genes appearing as hits i.e. causing localisation of Yap1p-GFP in the nucleus from the diethyl maleate screen were also genes that are regulated by Yap1p, namely TSA1 (thioredoxin peroxidase) and GPX2 (glutathione peroxidase).

Tsa1p is a thioredoxin peroxidise. It may catalyze the conversion of $\mathrm{ROS}$ to $\mathrm{H}_{2} \mathrm{O}$ and the corresponding alcohol, and thioredoxin facilitates the reduction of other proteins by cysteine thiol-disulfide exchange (Inoue, Matsuda et al. 1999). Tsa1p 
was shown to play an important role in the DNA integrity network (Pan, Ye et al. 2006). It is also essential for the transcriptional induction of other components of the thioredoxin system, TRX2 and TRR1, in response to $\mathrm{H}_{2} \mathrm{O}_{2}$ in a Yap1p/Skn7pdependent manner (Ross, Findlay et al. 2000). In fact, Fomenko et al. (2011) showed that thiol peroxidases play a role in regulating global gene expression in response to $\mathrm{H}_{2} \mathrm{O}_{2}$ by stimulating signalling through a MAP kinase pathway. They proposed that thiol peroxidases, including Tsa1p, act as redox sensors and oxidative stress signalling-transfer proteins. Therefore, Tsa1p contributes to the oxidative stress response by acting as a sensor and relaying the stress signal, as well as acting as a ROS scavenger (Fomenko, Koc et al. 2011).

Another Yap1p target gene that prevented Yap1p-GFP nuclear localisation in the presence of diethyl maleate when deleted is GPX2. GPX2 encodes phospholipid hydroperoxide glutathione peroxidase, which is a peroxide-scavenging enzyme induced by Yap1p and Skn7p (Ikner and Shiozaki 2005). GPX3 which is another glutathione peroxidase is involved in regulation of Yap1p in response to $\mathrm{H}_{2} \mathrm{O}_{2}$ induced oxidative stress but does not respond to either diamide or diethyl maleate. Gpx2p might act very similarly to Gpx3p in Yap1p regulation because overexpression of GPX2 can compensate for the loss of GPX3 to a certain extent and thioredoxin (TRX) is an endogenous electron donor for Gpx2p, similar to Gpx3p (Tanaka, Izawa et al. 2005). If this is true, then Gpx2p is possibly used by cells to distinguish between different oxidants by acting as a thiol-specific peroxidase in regulating Yap1p, probably via specific disulfide bonding in the $\mathrm{C}$ terminus of Yap1p.

Besides being involved in diamide, and diethyl maleate, GPX2 expression is greatly induced by calcineurin-mediated $\mathrm{Ca}^{2+}$ signalling (Tanaka, Izawa et al. 2005). A rise in intracellular $\mathrm{Ca}^{2+}$ and the presence of the oxidative stress-inducing $\mathrm{H}_{2} \mathrm{O}_{2}$, leads to leakage of mitochondrial content which in mammals results in induction of apoptosis (Valle, Fagian et al. 1993). In S. cerevisiae, it has been reported that Tsa1p (mentioned above as having a role in Yap1p regulation), and catalase help protect leakage of mitochondria induced by $\mathrm{Ca}^{2+}$ and $\mathrm{H}_{2} \mathrm{O}_{2}$ (Kowaltowski, Vercesi et al. 2000). In addition, Inoue et al., (1999) had shown that GPX2 and other glutathione gene expressions are increased in cells lacking Tsa1p. They proposed that increased glutathione was used as a backup system in the 
$\Delta t s a 1$ mutant, as well maintaining the catalytic cycle of glutathione peroxidase reaction (Inoue, Matsuda et al. 1999). It is therefore likely that Gpx2p, which can be used as a thioredoxin peroxidase backup system contributes to Yap1p regulation, especially in the presence of thiol oxidizing agents and $\mathrm{Ca}^{2+}$.

\section{Section VI: Yap1p-GFP screens and PDR screens}

Much literature has been devoted to showing crosstalk between the YAP1 oxidative stress response transcription factors and PDR ABC transporter genes (Wu, Wemmie et al. 1993; Miyahara, Hirata et al. 1996; Wendler, Bergler et al. 1997; Moye-Rowley 2003). However, it is still not clear how Yap1p is linked to PDR and if there is cross talk in regulation between these two stress responses.

From this point of view, we assessed the screen results from the primary PDR screen and Yap1p-GFP screens. We found that both PDR and Yap1p screens are enriched in genes functioning in transcriptional activation. However, the actual genes were different. PDR screens also contained more "hits" involved in kinasemediated signalling than the Yap1p screen. There was little overlap between redox sensitive screens and PDR screens, and none of the gene deletions identified from the PDR screen conferred any sensitivity toward 1, 1.5, 2 or $2.5 \mathrm{mM}$ of $\mathrm{H}_{2} \mathrm{O}_{2}$. One exception is deletion of KAP123, which showed slight sensitivity toward $2.5 \mathrm{mM} \mathrm{H}_{2} \mathrm{O}_{2}$. This observation suggested that different stress might utilise different "sensor" or regulators.

Three gene deletions were found in common for the PDR screens (i.e. preventing normal up-regulation of $A B C$-transporter Pdr5p and Yor1p) and the Yap1p diethyl maleate screen (i.e. preventing normal nuclear localisation of Yap1p). These were SSN2, SRB8 and KAP123. These genes function in gene regulation and nuclear transport. Both Ssn2p and Srb8p are mediator subunits which physically interact with Yap1p and with one another (Collins, Miller et al. 2007). However, the SSN2 deletion did not confer any sensitivity towards any compounds tested in the spot dilution assay (probably due to high redundancy in the PDR network). Srb8p, on the other hand, acts as a mediator for Pdr3p (Shahi, Gulshan et al. 2010), and causes an increase in xenobiotic sensitivity when deleted. The fact that SRB8 affects Yap1p nuclear localisation suggests that Srb8p might be a link between the PDR response and Yap1p mediated oxidative stress response. This point is 
possibly strengthened by the known negative genetic interaction with $B M H 1$ - one of the regulatory genes shown in the current work to be at the heart of the PDR response. Recent literature is now explicit that the post-translational modification of histones are part of specific signalling pathways triggered by specific stimuli, resulting in "context-dependent and specific" transcriptional activation of genes (Henry, Wyce et al. 2003; Smith and Shilatifard 2010; Bhaumik 2011). Therefore, we proposed that the chromatin remodelling complex and mediator complexes identified in the PDR and Yap1p screens have specific functions and are induced in PDR or oxidative stress-specific manner.

Based on the function of overlapped gene result, we propose that Yap1p may contribute to the PDR phenotype via the activation of other transcription factors, or through chromatin remodeling acting as a signal amplifier of an alternative pathway to PDR.

Another interesting point was that all the hits in common between the PDR and Yap1p screens were observed in the diethyl maleate screen. One explanation for this observation might be that diethyl maleate is a xenobiotic compared to $\mathrm{H}_{2} \mathrm{O}_{2}$, which is also produced internally. At the same time diethyl maleate also activates the oxidative stress response due to its ability to disrupt the cellular redox state resulting in oxidative stress response (Castillo, Ayté et al. 2002; Fomenko, Koc et al. 2011). We have not tried to use diethyl maleate to upregulate a PDR reporter, but it would be interesting to test this to see if the PDR response also is upregulated by this oxidant.

Another gene, PDE2, appeared in the GO enrichments of the $\mathrm{H}_{2} \mathrm{O}_{2}$ screens and might play role in both oxidative stress and the PDR response. PDE2 encodes a high-affinity cyclic AMP (CAMP) phosphodiesterase which inhibits protein kinase $A$ (PKA) (Sass, Field et al. 1986). The Ras-cAMP pathway regulates cAMPdependent PKA activity, which in turn regulates many cellular responses such as Skn7-mediated oxidative stress and hyperosmotic stress (Park, Grant et al. 2005). Overexpression of $P D E 2$ can restore oxidative stress tolerance to $\triangle y a p 1$ in the presence of $\mathrm{H}_{2} \mathrm{O}_{2}$ as well as stress tolerance in $\Delta m s n 2 \Delta m s n 4$ (Park, Grant et al. 2005). It seems likely that Pde2p might be involved in regulation of Yap1p and its alternative pathway involving Skn7p. The deletion of PDE2 also increases the 
Pdr5p-GFP reporter intensity in the PDR screens in non-stress conditions, nearly as much as RDR1 deletion which is a known suppressor of PDR5 (Hellauer, Akache et al. 2002) ( the data and discussion of deletions which upregulated Pdr5p is beyond the scope of this dissertation).

Taken together we suggest that Pde2p controls Yap1p via the PKA signalling pathway, perhaps assisting stress tolerance in the stationary phase (Pdr5p is found to be used for toxin elimination during exponential phase and is not highly expressed in the stationary phase). Since PDE2 only appeared as a hit under $\mathrm{H}_{2} \mathrm{O}_{2}$ exposure, $P D E 2$ might specifically regulate/modulate Yap1p in a $\mathrm{H}_{2} \mathrm{O}_{2}$ specific manner.

\section{Conclusion}

The fact that we found different sets of genes in the screens performed with $\mathrm{H}_{2} \mathrm{O}_{2}$ and with diethyl maleate, and no significant overlapped with the PDR screens, suggested that different regulators and/or modulators are required for Yap1p regulation in response to different oxidants or different type of stresses. These genes might also help cells to distinguish different oxidants depending on their mechanism of actions (Figure 5-8). Ross et al. (2000) showed that TRX2 seems to respond differently between low and high concentration of $\mathrm{H}_{2} \mathrm{O}_{2}$. The $\mathrm{C}$-terminal region of Yap1p is the major nuclear localisation control but other regions of the protein have also been suggested to play role in the regulation of Yap1p in oxidative stress responses (Wemmie, Steggerda et al. 1997). The C-terminal, with the combination with different subunits of RNA polymerase II mediator complex, chromatin modification complex, and redox target genes (Tsa1p, Gpx2p, Gpx3p) might serve as sensors for different oxidants. The role of the Yap1p N-terminus and $\mathrm{C}$-terminus and their interaction with any regulatory proteins as a way to regulate Yap1p still needed further study. In addition, we identified PDE2 as a potential regulator of Yap1p in the presence of $\mathrm{H}_{2} \mathrm{O}_{2}$, and deletion of this gene also increased Pdr5-GFP reporter intensity. Pde2p might play role as a negative regulator for the PDR response and positive regulator in the Yap1p response to $\mathrm{H}_{2} \mathrm{O}_{2}$. 


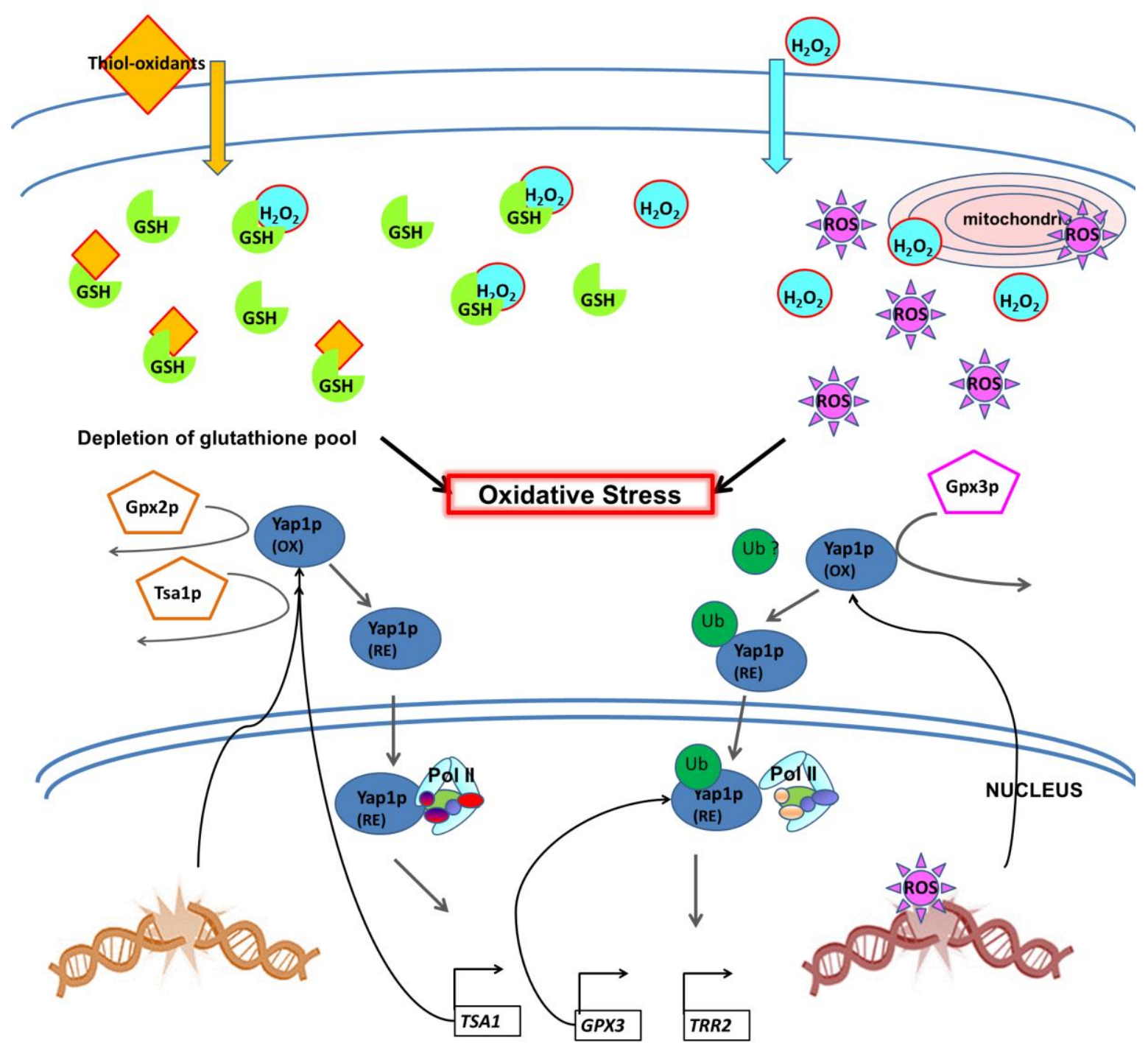

Figure 5-8: The diagram shows that different sets of genes may be used to regulate the Yap1p response to different oxidative stress-inducing agents.

Depending on how oxidative stress is generated, different sets of regulators are activated. $\mathrm{H}_{2} \mathrm{O}_{2}$ directly generates ROS and seems to be regulated via ubiquitination. Diamide and diethyl maleate cause oxidative stress by depleting GSH pool and seem to be regulated by Gpx2p and Yap1p target gene products (Tsa1p) i.e. negative feedback. 


\section{Chapter 6. Final Discussion and Conclusion}

\section{Section I: New Methodologies}

We have generated genomic tools which allowed us to investigate a set of biological processes (and complex traits) relating to environmental bioctives, namely, multidrug resistance mechanisms, oxidative stress response and drug mode-of-action.

\section{(a) Modification of SGAM and application of chemical-genetic profile to identify drug target and mode-of-action}

The first two processes we explored to do with xenobiotics, namely, PDR and oxidative stress, overlap in function and are used to export xenobiotics by ABCtransporters conferring multi drug resistance (Balzi and Goffeau 1995; Gulshan and Moye-Rowley 2007; Kuchler and Schüller 2007). This approach leaves open questions about xenobiotics/drugs that are not $\mathrm{ABC}$-transporter substrates. How do cells detoxify these compounds? Many well-known antifungal drugs such as amphotericin and nystatin, are not ABC-transporter substrates and yet resistance against these two drugs also has been observed whose targets are complex and resistance not easy to explain. In Chapter 3, we identified a drug target mechanism in part by modifying the SGAM method (Jorgensen, Nelson et al. 2002) in combination with the information obtained from the genome-wide chemicalgenetic profiling to identify the target and study the mode-of action of a non-efflux pump substrate with antifungal property, neothyonidioside (neo).

In our use of SGAM methodology (described in Yibmantasiri et al 2012), we treated a drug resistance locus as an antibiotic marker and used the deletion mutant array as a high density genetic map. Although SGAM has been used to identify a genetic mutation (Jorgensen, Nelson et al. 2002), it has not been applied to identify a drug resistant locus as we have done. We narrowed down the potential neo resistant genes to five essential genes which appeared in the centre of the linkage group of gene deletions that surrounded the neo (drug) resistant locus. Based on the enrichment of ESCRT and sterol genes from the neo chemical genetic profile, we applied a candidate gene approach and sequenced NCP1, an NADP-cytochrome P450 reductase involved in ergosterol biosynthesis which showed a key mutation blocking ergosterol synthesis at lanosterol (Yibmantasiri et 
al 2012). In that paper, we presented good evidence that neo must disruptively bind to egosterol but only above certain threshold concentrations of ergosterol. Because of the ESCRT gene involvement we hypothesized that neo disrupts vesicle formation, which is an essential process of cells. We used confocal microscopy to investigate the mode-of-action of neo in more detail. We found that a sublethal concentration of neo $(0.8 \mu \mathrm{M})$ prevents normal vacuole formation (Figure 3-2). Given the fact the neo resistant strain has less cellular ergosterol (Yibmantasiri, Leahy et al. 2012) and that there is a threshold effect of egosterol at which neo is toxic we proposed that neo disrupts vacuole formation by disrupting membrane fluidity when it binds to ergosterol in plasma membrane. Identifying the neo resistance mutation was a central part of the logic leading to this conclusion and central to the mutation identification was the modified use of the genomic tool, SGAM. It is unlikely the conclusion on the target of neo, i.e. membrane fluidity via egosterol binding, could have been reached without the use of genomic tools as described here.

\section{(b) Development of genome-wide high content microscopy screen with fluorescent reporters}

The first genomic tool we generated was the reporter gene system linked with green fluorescent protein (GFP) which was then introduced into the $S$. cerevisiae non-essential gene deletion set. This genome-wide deletion-reporter system was then used to systematically explore biological processes of interest by measuring fluorescence intensity and object texture at subcellular locations from images captured by an automatic high-content fluorescent microscope (Opera).

In the first reporter system, Pdr5p and Yor1p, ABC transporters found in many cells showing the multidrug resistant phenotype (Kuchler and Schüller 2007), were expressed as fusion proteins linked to GFP and served as real-time indicators of transcriptional activity of Pdr1p and Pdr3p. Both Pdr1p and Pdr3p are the main transcription factors controlling the multidrug resistant response to xenobiotics, known as pleiotropic drug resistance (PDR) in yeast. We used this reporter to search for "upstream" genes involved in sensing xenobiotics thence delivering signals to the Pdr1p/Pdr3p transcription factors. It became clear during this search that modulators and/or genes, which provide alternative pathway for the PDR phenotype, also were identified. An assumption behind this reporter-based search 
was that deletions that failed to up-regulate both reporters at the cell surface in the presence of atorvastatin could be identified as potential modulators of Pdr1p/Pdr3p.

The second genome-wide high-content microscopy reporter system we developed was the Yap1p-GFP reporter, a basic leucine zipper (bZIP) transcription factor required for oxidative stress tolerance (Moye-Rowley, Harshman et al. 1989). In order to identify genes involved in Yap1p regulation in response to different oxidants, we screened for gene deletions that prevented the normal nuclear localization of the reporters upon oxidative stress encounter.

These two reporter systems are, to our knowledge, the first reporters which combine high-content microscopy with genome wide screening such that far more resolution of the cell reactions during stress, beside growth, can be studied. These screens thus allowed us to identify deletions that only give subtle changes that probably would not be detected by a growth assay alone. Both reporter systems provided reproducible results over numerous screen repeats. The screens received independent validation when deletion mutants from the PDR primary screens were subjected to spot dilution and Western blot assays.

\section{Section II: New gene functions identified in these studies by genomic approaches}

\section{(a) Enrichment of ESCRT genes in the non-efflux-pump substrate neothyonidioside}

In our published study, we performed a chemical-genetic homozygous deletion profiling (HOP) assay (Yibmantasiri, Leahy et al. 2012). Analysis of GO term enrichment of the neo profile, pinpointed the ESCRT complexes as essential components for neo resistance. All of the members of ESCRT-II (VPS25, VPS36, SNF8), some components of ESCRT-0 (VPS27), ESCRT-I (STP22, VPS28), and ESCRT-III (SNF7, VPS20) and VPS4, an ATP-ase that drives the activity of the ESCRT-III complex as well as deletion mutants of the GARP complex (VPS51 and VPS52) and vesicle transport genes (VPS5, VPS41, VPS17), also endocytosis gene deletions (END3, CAP1, RVS167, RVS161) become essential in the 
presence of neo (Figure 3-1). We proposed that Neo blocks multivesicular body and vacuole formation via disruption of membrane fluidity.ESCRT genes compensate Neo toxicity by providing aid to membrane inward budding

The enrichment of vesicle-related genes and ESCRT complex genes from the chemical genetic profile was also observed with polyenes, nystatin and amphotericin (Parsons, Brost et al. 2004; Parsons, Lopez et al. 2006) but not as strongly as with neo. It has been shown that cells use lysosomes to detoxify drugs that can lead to PDR phenotypes (Kuchler and Schüller 2007). We propose that disruption of plasma membrane sterol distribution by these compounds leads to initiation of signal transduction, which in turn activates the PDR response. The suggestion here is that this response might also involve ESCRT complex and vacuole formation, especially when cells encounter drugs that are not $A B C$ transporter substrates.

\section{(b) Enrichment of genes involved in chromatin modification, RNA} polymerase II mediator complexes, and transcription factors in PDR screens Chromatin remodelling complexes and co-activators such as RNA polymerase II mediator subunits, affect transcriptional activity of gene expression globally (Hahn and Young 2011; Niederacher, Klopf et al. 2011). However, these functions also occur for specific gene expression ("chromatin signalling pathway") (Smith and Shilatifard 2010; Hnisz, Tscherner et al. 2011). Chromatin modification/remodelling complexes have been associated with the drug resistant phenotype in $S$. cerevisiae, C. albicans, and mammalian breast cancer cell lines (Gao, Wang et al. 2004; Morschhäuser, Barker et al. 2007; Toth, Boros et al. 2012). In the current studies we identified such genes encoding components of SAGA complexes and transcriptional activators relating to the PDR response. Since none of the deletions conferred sensitivity toward $\mathrm{H}_{2} \mathrm{O}_{2}$, we suggest that these genes are likely to be PDR specific modulators and different mediators and chromatin modifications are used to differentiate different stress responses. These gene activities are likely to be specific and not global. Deletions of SPT7, ROX3, SPT8, and DEP1 for example decrease Pdr5p abundance in Western blots without affecting another plasma membrane protein Pma1p. 
Such "chromatin signalling pathways" discussed above can regulate gene expression in host-pathogen interactions as well as modulate drug resistance trait by changing histone modification status (Lopez-Rubio, Riviere et al. 2007; Hnisz, Tscherner et al. 2011). It has been shown that the histone acetylase complex can alter cAMP/PKA signalling which then affect the virulence of $C$. albicans (Hnisz, Tscherner et al. 2011). Recent studies show that novel histone acetylase /decetylase inhibitors reduce virulence of many pathogenic fungi (Simonetti, Passariello et al. 2007; Agbor-Enoh, Seudieu et al. 2009; Wurtele, Tsao et al. 2010). These pathways may provide new targets for overcoming drug resistance, especially if the drug resistant phenotype is achieved from mechanisms other than hyperactive Pdr1p/Pdr3p. Compound library screening using these deletion backgrounds and looking for compound sensitivity should provide more insight of how these genes might work together to influence PDR phenotype.

(c) Different genes involved in regulating Yap1p nuclear localization in different oxidants

Our results show that different sets of genes control Yap1p upon application of different oxidants. Nevertheless, the broad functions of these genes were very similar. Genes that are involved in Yap1p regulation in both $\mathrm{H}_{2} \mathrm{O}_{2}$ and diethyl maleate treatments were enriched in DNA integrity, and stress response. We suggest that Yap1p might be regulated by ubiquitination in an $\mathrm{H}_{2} \mathrm{O}_{2}$-specific manner since ubiquitination genes were enriched in this oxidant screen. We also showed that the known Yap1p targets such as TSA1 (encodes thiol peroxidase), and GPX2 (encodes glutathione peroxidise) were also involved in Yap1p regulation, probably via their ability to sense and regulate the redox state of the cells which in turn change the redox state of Yap1p (Yap1p in the reduced state is localized to the nucleus). We propose that Gpx2p assists oxidation and activation of Yap1p in a thiol-oxidant specific manner. In a similar way, Gpx3p activates Yap1p when exposed to $\mathrm{H}_{2} \mathrm{O}_{2}$.

\section{Section III: Predicting and verifying genes from network information}

As is implicit in much of the above discussion, genomic approaches to defining gene function are hypothesis forming. A testable one is that known negative 
genetic and physical interactions of specific genes arising from our PDR screen "hits" that fall into similar GO categories will affect down-stream reporters in a similar way. If in fact this can be shown, the prediction and its verification serves to validate the supposed function of the original hits and better defines the interaction-associated genes. To this end we curated interacting genes from SGD that interact with $P D R 1$ and PDR3 (both physically and genetically) and with highly-interacted GO nodes from our results (SAGA, kinase, lipids). We tested these genes by spot dilution of specific deletions both as $\Delta x x x$ and in the $\Delta p d r 1$ $\Delta p d r 3$ background in our xenobiotic compound panel and by abundance of Pdr5p in Western blots. We found that 10/18 mutants appeared as additional hits by one or other of these assays. These genes, listed in Table 4-4, are enriched in chromatin remodelling. The "hits' were gene specific i.e. likely to be non-global as their deletion did not affect the abundance of Pma1p in plasma membrane in Western blots; neither did they cause sensitivity toward $\mathrm{H}_{2} \mathrm{O}_{2}$. Unexamined in the foregoing discussion is the question of the function of genetic networks. Indeed this comment may apply to the now growing literature on genetic interaction networks, starting with yeast but increasingly seen in other species as key genetic tools such as genome-scale deletion sets of genes become available. The results reported here do, in fact, begin to answer that question by experimentally showing that gene deletions additional to primary hubs of networked genes can also affect the phenotypes controlled by the hubs. One example from our results that utilised network function is $L G E 1$, a gene that encodes a protein of unknown function. $L G E 1$ fell short of the "cut off" to be identified as a "hit" in the primary screen. We supposed that deletion of LGE1 might affect the PDR phenotype because LGE1 displayed phenotypic suppression PDR3 and is synthetic lethal with SWI4, and Ume6p. As shown in Chapter 4, deletion of LGE1 did not increase xenobiotic sensitivity in any deletion backgrounds. However, it did reduce Pdr5p abundance. This effect might due to an LGE1 function being associated with the network hub $P D R 3$, SWI4 and UME6, engaged in transcriptional regulation. By assessing the network, we can ascertain the pathway in which Lge1p might work. This adds a dimension to understanding complex phenotypes, which usually have been couched in classical genetic terms of the "additive" and "epistatic" contributions of multiple genes measured by the tools of quantitative genetics. 


\section{Section IV: New pathways contribute to PDR network}

\section{(a) Kinase signalling pathway relay xenobiotics information and activate PDR response}

We identified 26 gene deletions that prevented normal up-regulation of Pdr5p and Yor1p and conferred sensitivity to several xenobiotic compounds. Many of these genes are involved in signalling (Rho-GTPase, MAPK). Since the gene deletions appeared not to be involved in transporter location we propose that there is signalling "upstream" of Pdr1p/Pdr3p which might also be involved in other stress responses and work in combination with chromatin remodelling complexes and transcriptional activators.

\section{(b) Lipids, mitochondria and PDR}

The screening results reported in this thesis also highlighted the importance of the lipid homeostasis and mitochondria in PDR responses. Deletion of mitochondria matrix proteins such as FMC1 or genes encoding phospholipid or sphingolipid DEP1, YME1, PIB2 resulted in hypersensitivity to many of the xenobiotic compounds greater than that displayed by the $\Delta p d r 1 \Delta p d r 3$ strain showing these were additional effects to those regulated by Pdr1p/Pdr3. This result is seemingly at odds with the known upregulation of Pdr5p in $\rho^{0}$ (mitochondria genomedeleted/defected) cells that is Pdr3p-dependent (Hallstrom and Moye-Rowley 2000; Moye-Rowley 2005; Shahi, Gulshan et al. 2010). Were they involved in the same pathway deletion of these mitochondrial genes should not cause extra sensitivity in the $\Delta p d r 1 \Delta p d r 3$ strain. An explanation may lie with sphingolipid precursors that play a role in signalling and link with mitochondrial status and stress tolerance (Sietsma, Veldman et al. 2001), suggesting that some of the genes involved in sphingo/phospholipid synthesis might also play roles in different (to Pdr1p/Pdr3) stress responses that can also upregulate $A B C$-transporter gene expression.

The importance of lipid composition and structure also emerged from our study of neo dose response in different erg mutants (Kristina Boeger's summer research report). We showed in chapter 5 that neo, a sterol binder, can have "preferable" substrate(s). Ergosterol and some precursors bind to neo whilst episterol (substrate for Erg3p) does not. This conclusion can be deduced from the fact that 
only the deletion of ERG3 amongst erg mutants caused increase in resistance toward neo. It could be useful to use this information in the design of compounds that target more specific sterols. Then, used in combination with known clinical drugs to increase cell susceptibility to combat drug resistance in both pathogenic fungi as well as in cancer cells.

\section{(c) Possible pathways to achieve PDR phenotypes. Lessons from our genomic studies}

From our combined results, we propose that cells encountering xenobiotics or other drug-like compounds (Figure 6-1) initiate a signalling pathway. The initiation might arise from compounds directly binding to sterol in the plasma membrane as in the case of neo and polyenes, or maybe via interaction with phospholipid or other protein (e.g. Chw43p, our hit) in lipid rafts (Hallstrom, Lambert et al. 2001). From our results, signalling pathways might involve PKC and/or Ras/MAPK which have been shown to play a role in stress responses by activation of Skn7p (another TF for oxidative stress) and Msn2p/Msn4p in translocating these transcription factors to the nucleus on encountering stress (Krems, Charizanis et al. 1996; Görner, Durchschlag et al. 1998; Hanlon, Rizzo et al. 2011). This signalling cascade might also activate other proteins involved in post translational modification such as Bmh1p, an important hit and one of the main network hubs from our PDR screens. Bmh1p (14-3-3 protein) controls many processes including exocytosis, vesicle transport, and Ras/MAPK signalling (van Heusden, Wenzel et al. 1992; Bruckmann, Hensbergen et al. 2007). Bmh1p is found in both the nucleus and plasma membrane and can bind both proteins and DNA (www.yeastgenome.org). We suggest it is a "decision junction" distinguishing xenobiotics as pump and non- pump substrates. In this view, compounds can either be pumped out of the cell using ABC-transporters and major facilitators (MFS), or, alternatively, transported to vacuoles and lysosome. Especially, when the compounds are not PDR substrates (e.g polyenes, neo). It has been shown that 14-3-3 proteins are required as a scaffold for the enhancer proteins to bind at the promoter (Kellner, Ramos et al. 2012). This activation process involves the mediator complex and chromatin modification complexes which might act as sensors for signalling (kinases) and responses of chromatin-signalling pathways (Smith and Shilatifard 2010). 
It is possible different subunits of the mediator complex as well as chromatin modification complex and transcription activators are used to activate transcription factors other than PDR1/PDR3 depends on compounds mechanism of actions. It is also possible that other such transcription factors function as amplifiers for Pdr1p/Pdr3p signal. Zheng et al. 2010 showed that genes can contain many recognition sequences belonging to different transcription factors. Some of these are acting as major regulators and some as minor regulators. In the absence of major regulators, the minor regulators could become more important and deletion of minor regulators could then cause more severe phenotypic effects (Zheng, Benschop et al. 2010). The same authors also showed that there is a strong preference for negative genetic interaction between genes encoding specific DNAbinding transcription factors (STFs), especially between STFs which show overlapping targets (Zheng, Benschop et al. 2010). These findings and interpretations are consistent with our PDR screen results. Many gene deletions that showed more severe sensitivity towards many xenobiotics in the absence of PDR1 PDR3 clearly implicate that they are involved drug response processes that are not the classical PDR response (or Pdr1p/Pdr3p-dependent). To be considered also is that activation of transcription factors can also lead to activation of other transcription factors, for example, Pdr1p activates Pdr3p, and Yrr1p; $P D R 3$ and YRR1 also are autoregulated.

The targets of the transcription factors can also act as regulators of transcription factors as can be seen from the diethyl maleate screen result. Tsa1p and Gpx2, both Yap1p targets, can also act as sensors for the change of cellular redox state and relay information to Yap1p, which leads to nuclear localization of Yap1p. Chromatin modification, SAGA, and members of mediator complexes provide cells with another way to sense and relay stress signals besides post-translational modification of proteins. 


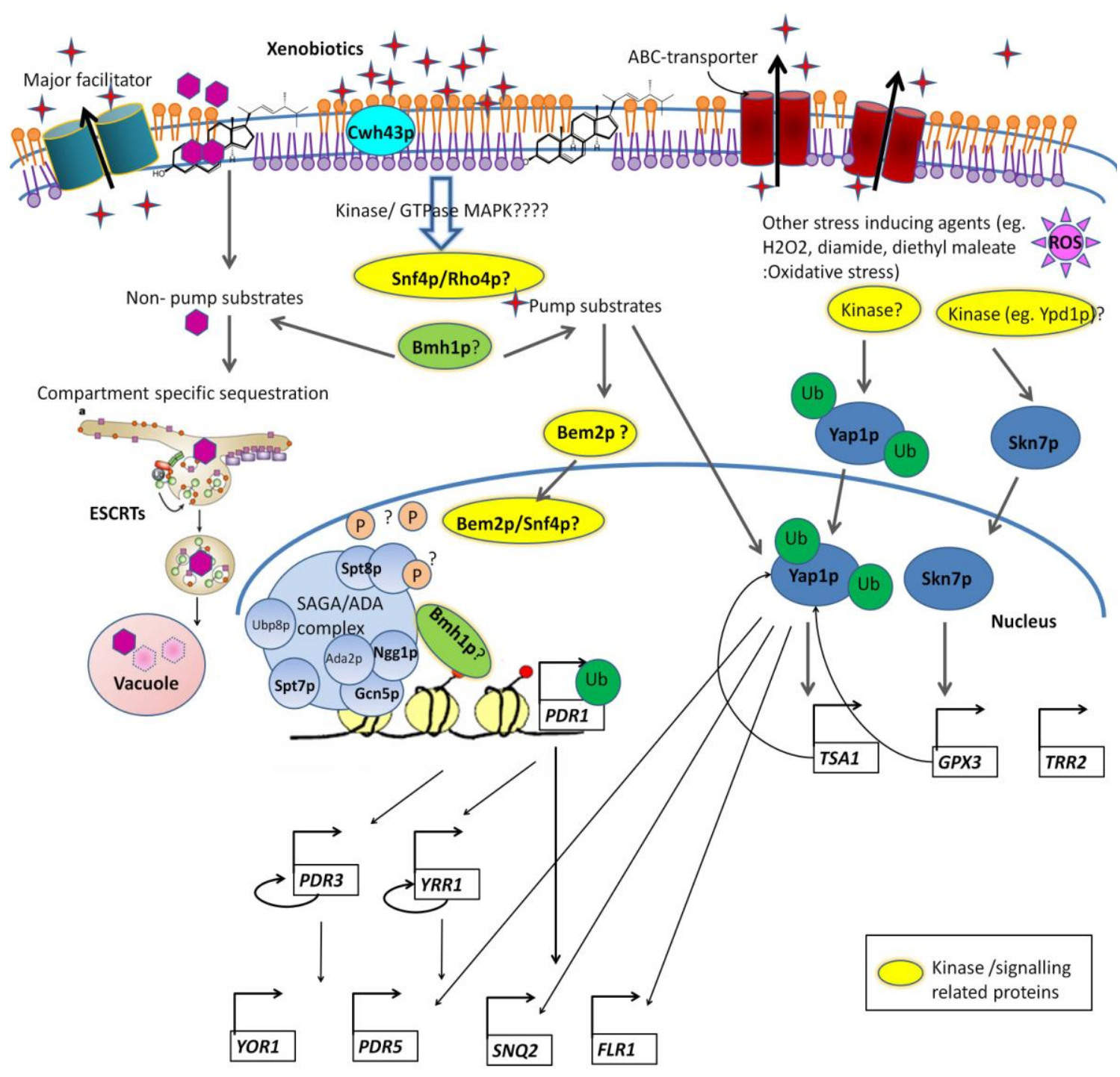

Figure 6-1 : Diagram representing possible pathways in S.cerevisiae activated by xenobiotics

It is very likely that kinase signalling cascade, ubiquitination and change in context-specific chromatin modification are involved in distinguishing between different substrates.

\section{Section V: Comparison of our results to relevant key papers}

If our hits act in the same pathway as Pdr1p/Pdr3p, then our gene deletions should also appeared in the MDR list of Hillenmeyer chemical-genetic screens of 178 drugs (Hillenmeyer, Fung et al. 2008). We found that out of 54 gene deletions we identified as causing hypersensitivity to drugs, 9 of them overlapped with the Hillenmeyer MDR list (deletions of BMH1, PDR, SKN7, RCY1, PIN4, CWH43, ERV14, FIS1, and OPI3). If pathways we proposed above are correct, Bmh1p 
plays a major role as a sensor and signalling, and therefore acts upstream of Pdr1p/Pdr3p and should contribute to the PDR phenotype; BMH1 was a hit in the Hillenmyer screen. However, none of our chromatin remodelling complex candidates, RNA polymerase II mediator candidate genes, and kinase genes overlapped with the Hillenmeyer et al. MDR list. This discrepancy suggests that, either we have characterised alternative pathways that have not yet been identified or that different screening systems inherently produce different results. The latter is a possibility because Hillenmeyer et al. used diploid cells which could lead to epistatic effects between genes masking gene deletions. Furthermore, the Hillenmeyer et al. screens were growth screens, whilst ours are reporter systems which probably can screen for more subtle changes. Another difference is that our reporter systems are set up so that Pdr1p/Pdr3p and their targets remain intact and that the effect of each gene deletion is individually assessed, whilst the Hillenmeyer et al. screen is based on competitive fitness of all the deletion mutants assayed together.

Another paper key paper for discussion is Barreto et al 2011, who used genome wide screens for changed tolerance to cationic drugs that affect potassium homeostasis (Barreto, Canadell et al. 2011). They found nearly the same set of SAGA complex genes as described here (SPT7 GCN5, NGG1), however they thought the genes were part of a pleiotropic "global" effect and did not discussed this result further. We, however, think that the effects of these SAGA gene deletions are not merely a global effect since they affected one plasma membrane protein (Pdr5p) and not another (Pma1p).

\section{Section VI: Power of Genomics}

We showed here that unbiased genome-wide approaches can identify previously unsuspected regulators of complex traits of interest, namely drug mode-of-action and development of drug resistance and stress response.

The techniques of SGAM and chemical-genetic profiling generate results allowing specific hypotheses to test for the mode-of-action of a drug (neo in this study) could be. Without these hypothesis-generating methodologies we would have no clue where to start and it would take much longer to come up with the significant results - if at all. 
By using the genome-wide reporter system that we developed we could explore all the non-essential gene contributions to most biological processes of interest. In the case of PDR and Yap1p, the reporters provide reproducible results validated by other tests and can be used to develop networks of gene functionality around the biological process in question. We found that there are signalling pathways upstream of Pdr1p/Pdr3p and Yap1p. Additionally, we found that different "sensors" and regulators are induced upon exposure to different xenobiotic stresses. To our To our knowledge, it is the first genome-wide reporter system that allows us to measure intra-cellular phenotypes as well as the localization of the reporters in large numbers of individual cells in a genome-wide manner for the PDR response and the oxidative stress response. In a methodological advance, texture analysis from Acapella software allowed us to look at texture around the cellular organelle of interest (here plasma membrane in PDR reporter, cytoplasm and nucleus in Yap1p) providing greater quantitative accuracy than just measuring GFP intensity. By observing intracellular morphology of the cells in the deletion screens, we were able to distinguish between gene deletions that have "upstream" effects on Pdr1pPdr3p TF activity (and hence Pdr5p expression), and gene deletions that affect PDR GFP reporter location internally. Examples of the latter are gene deletions that disrupt protein folding or that Pdr $5 p / Y o r 1 p$ reporters are localized in cytoplasm or in the ER instead of plasma membrane. Without microscopy, these deletions would present as false positives in our screen. This is another advantage of high-content microscopy over FACS.

\section{Future Direction}

A useful line of enquiry would be to study genes involved in a pre-PDR1/PDR3 signalling pathway, as well as some SAGA complex genes. Subunits of SAGASWI complexes have been shown to have negative genetic interactions with many of the RNA polymerase II mediator complex genes, suggesting that they can compensate one another. For example, GAL11 genetically interacts with SPT8 and SWI4 (Collins, Miller et al. 2007), hence, to a certain extent, the requirement of Gal11p for PDR can be bypassed. In vitro biochemical assays could be conducted to test this hypothesis in which all the components of transcriptional activation are added except Gal11p. In theory, this should reduce the expression of Pdr5p. This defect should be able to be rescued by adding Swi4p and/or Spt8p 
back unless Swi4p and Spt8p compensate the loss of Gal11p by alternative pathways involving other $\mathrm{ABC}$-transporters all together, or independent of Pdr1p/Pdr3p.

We would also like to look at ubiquination as a regulator for Yap1p and stress response both oxidative stress and possible crosstalk with PDR.

We would like to look at the gene deletions which in normal growth conditions upregulate Pdr5p and Yor1p relative to wild-type level such as PDE2, which has been mentioned briefly in Chapter 5 . These genes should, in theory, be the negative regulator of the PDR pathway. We obtained these data, however due to the time limitation we did not validate these data. From the GO analysis at $5 \%$ level of significance, we found enrichment in processes of oxidation-reduction, chemical homeostasis, response to stimuli and xenobiotic stimulus and regulation of protein kinase activity. We would like to validate these data with spot dilution assay and western blot and if any genes show particular interest, to investigate them in more detail. A major question arising out of this work is, do Pdr1p/Pdr3p act at the same time or sequentially because Pdr1p can form both homodimers and heterodimers? Will mediators determine the sequence and/or what subsets of target genes are turned on? 


\section{References}

Agbor-Enoh, S., C. Seudieu, et al. (2009). "Novel Inhibitor of Plasmodium Histone Deacetylase That Cures P. berghei-Infected Mice." Antimicrobial Agents and Chemotherapy 53(5): 1727-1734.

Akache, B., S. MacPherson, et al. (2004). "Complex Interplay Among Regulators of Drug Resistance Genes in Saccharomyces cerevisiae." Journal of Biological Chemistry 279(27): 27855-27860.

Alarco, A.-M., I. Balan, et al. (1997). "AP1-mediated Multidrug Resistance in Saccharomyces cerevisiae Requires FLR1 Encoding a Transporter of the Major Facilitator Superfamily." Journal of Biological Chemistry 272(31): 19304-19313.

Albertsen, M., I. Bellahn, et al. (2003). "Localization and Function of the Yeast Multidrug Transporter Tpo1p." Journal of Biological Chemistry 278(15): 12820-12825.

Artal-Sanz, M., L. de Jong, et al. (2006). "Caenorhabditis elegans: A versatile platform for drug discovery." Biotechnology Journal 1(12): 1405-1418.

Arthington, B. A., L. G. Bennett, et al. (1991). "Cloning, disruption and sequence of the gene encoding yeast C-5 sterol desaturase." Gene 102(1): 39-44.

Avilov, S. A., A. I. Kalinovskii, et al. (1990). "New triterpene glycoside from the holothurian\&lt;i\&gt;Neothyonidium magnum\&lt;/i\&gt." Chemistry of Natural Compounds 26(1): 42-45.

Babiychuk, E., S. Kushnir, et al. (1995). "Arabidopsis thaliana NADPH Oxidoreductase Homologs Confer Tolerance of Yeasts toward the Thioloxidizing Drug Diamide." Journal of Biological Chemistry 270(44): 2622426231.

Baetz, K., L. McHardy, et al. (2004). "Yeast genome-wide drug-induced haploinsufficiency screen to determine drug mode of action." Proceedings of the National Academy of Sciences of the United States of America 101(13): 4525-4530.

Balkis, M. M., S. D. Leidich, et al. (2002). "Mechanisms of Fungal Resistance: An Overview." Drugs 62(7): 1025-1040.

Balzi, E., W. Chen, et al. (1987). "The Multidrug resistance Gene PDR1 from Saccharomyces cerevisiae." The Journal of Biological Chemistry 262(December 15): 16871-16879.

Balzi, E. and A. Goffeau (1995). "Yeast multidrug resistance: The PDR network." Journal of Bioenergetics and Biomembranes 27(1): 71-76.

Barreto, L., D. Canadell, et al. (2011). "A Genomewide Screen for Tolerance to Cationic Drugs Reveals Genes Important for Potassium Homeostasis in Saccharomyces cerevisiae." Eukaryotic Cell 10(9): 1241-1250.

Bauer, B. E., D. Rossington, et al. (2003). "Weak organic acid stress inhibits aromatic amino acid uptake by yeast, causing a strong influence of amino acid auxotrophies on the phenotypes of membrane transporter mutants." European Journal of Biochemistry 270(15): 3189-3195.

Bauer, B. E., H. Wolfger, et al. (1999). "Inventory and function of yeast ABC proteins: about sex, stress, pleiotropic drug and heavy metal resistance." Biochimica et Biophysica Acta (BBA) - Biomembranes 1461(2): 217-236.

Bauman, J. W., J. M. McKim Jr, et al. (1992). "Induction of metallothionein by diethyl maleate." Toxicology and Applied Pharmacology 114(2): 188-196. 
Bhaumik, S. R. (2011). "Distinct regulatory mechanisms of eukaryotic transcriptional activation by SAGA and TFIID." Biochimica et Biophysica Acta (BBA) - Gene Regulatory Mechanisms 1809(2): 97-108.

Biggin, Mark D. (2011). "Animal Transcription Networks as Highly Connected, Quantitative Continua." Developmental Cell 21(4): 611-626.

Bircham, P. W., D. R. Maass, et al. (2011). "Secretory pathway genes assessed by high-throughput microscopy and synthetic genetic array analysis." Molecular BioSystems 7(9): 2589-2598.

Björklund, S. and C. M. Gustafsson (2005). "The yeast Mediator complex and its regulation." Trends in Biochemical Sciences 30(5): 240-244.

Bossche, H. V., M. Engelen, et al. (2003). "Antifungal agents of use in animal health - chemical, biochemical and pharmacological aspects." Journal of Veterinary Pharmacology and Therapeutics 26(1): 5-29.

Botstein, D., S. A. Chervitz, et al. (1997). "Yeast as a Model Organism." Science 277(5330): 1259-1260.

Boutros, M. and J. Ahringer (2008). "The art and design of genetic screens: RNA interference." Nat Rev Genet 9(7): 554-566.

Bradner, J. E., O. M. McPherson, et al. (2006). "A Robust Small-Molecule Microarray Platform for Screening Cell Lysates." Chemistry \&amp; Biology 13(5): 493-504.

Brôco, N., S. Tenreiro, et al. (1999). "FLR1 gene (ORF YBR008c) is required for benomyl and methotrexate resistance in Saccharomyces cerevisiae and its benomyl-induced expression is dependent on Pdr3 transcriptional regulator." Yeast 15(15): 1595-1608.

Brown, J. L., S. North, et al. (1993). "SKN7, a yeast multicopy suppressor of a mutation affecting cell wall beta-glucan assembly, encodes a product with domains homologous to prokaryotic two-component regulators and to heat shock transcription factors." Journal of Bacteriology 175(21): 6908-6915.

Bruckmann, A., P. J. Hensbergen, et al. (2007). "Post-Transcriptional Control of the Saccharomyces cerevisiae Proteome by 14-3-3 Proteins." Journal of Proteome Research 6(5): 1689-1699.

Burbelo, P. D. and A. Hall (1995). "14-3-3 Proteins: Hot numbers in signal transduction." Current Biology 5(2): 95-96.

Burston, H. E., L. Maldonado-Báez, et al. (2009). "Regulators of yeast endocytosis identified by systematic quantitative analysis." The Journal of Cell Biology 185(6): 1097-1110.

Carpenter, A. E. and D. M. Sabatini (2004). "Systematic genome-wide screens of gene function." Nat Rev Genet 5(1): 11-22.

Carvajal, E., H. B. van den Hazel, et al. (1997). "Molecular and phenotypic characterization of yeast PDR1 mutants that show hyperactive transcription of various ABC multidrug transporter genes." Molecular and General Genetics MGG 256(4): 406-415.

Casamassimi, A. and C. Napoli (2007). "Mediator complexes and eukaryotic transcription regulation: An overview." Biochimie 89(12): 1439-1446.

Castillo, E. A., J. Ayté, et al. (2002). "Diethylmaleate activates the transcription factor Pap1 by covalent modification of critical cysteine residues." Molecular Microbiology 45(1): 243-254.

Causton, H. C., B. Ren, et al. (2001). "Remodeling of Yeast Genome Expression in Response to Environmental Changes." Molecular Biology of the Cell 12(2): 323-337. 
Chen, K.-H., T. Miyazaki, et al. (2007). "The bZip transcription factor Cgap1p is involved in multidrug resistance and required for activation of multidrug transporter gene CgFLR1 in Candida glabrata." Gene 386(1-2): 63-72.

Chung, K., M. M. Crane, et al. (2008). "Automated on-chip rapid microscopy, phenotyping and sorting of C. elegans." Nat Meth 5(7): 637-643.

Ciechanover, A. (1994). "The ubiquitin-mediated proteolytic pathway: mechanisms of action and cellular physiology." Biological chemistry Hoppe-Seyler 375(9): 565-581.

Ciechanover, A. and K. Iwai (2004). "The Ubiquitin System: From Basic Mechanisms to the Patient Bed." IUBMB Life 56(4): 193-201.

Cohen, B. A., Y. Pilpel, et al. (2002). "Discrimination between Paralogs using Microarray Analysis: Application to the Yap1p and Yap2p Transcriptional Networks." Molecular Biology of the Cell 13(5): 1608-1614.

Coleman, S. T., E. A. Epping, et al. (1999). "Yap1p Activates Gene Transcription in an Oxidant-Specific Fashion." Molecular and Cellular Biology 19(12): 8302-8313.

Collins, S. R., K. M. Miller, et al. (2007). "Functional dissection of protein complexes involved in yeast chromosome biology using a genetic interaction map." Nature 446(7137): 806-810.

Costanzo, M., A. Baryshnikova, et al. (2010). "The Genetic Landscape of a Cell." Science 327(5964): 425-431.

Craig, K. L. and M. Tyers (1999). "The F-box: a new motif for ubiquitin dependent proteolysis in cell cycle regulation and signal transduction." Progress in Biophysics and Molecular Biology 72(3): 299-328.

Cui, Z., D. Hirata, et al. (1996). "The Multidrug Resistance-associated Protein (MRP) Subfamily (Yrs1/Yor1) of Saccharomyces cerevisiae Is Important for the Tolerance to a Broad Range of Organic Anions." Journal of Biological Chemistry 271(25): 14712-14716.

Cui, Z., T. Shiraki, et al. (1998). "Yeast gene YRR1, which is required for resistance to 4-nitroquinoline $\mathrm{N}$-oxide, mediates transcriptional activation of the multidrug resistance transporter gene SNQ2." Molecular Microbiology 29(5): 1307-1315.

Davidson, E. H. (2010). "Emerging properties of animal gene regulatory networks." Nature 468(7326): 911-920.

Davis, N., J. Horecka, et al. (1993). "Cis- and trans-acting functions required for endocytosis of the yeast pheromone receptors." The Journal of Cell Biology 122(1): 53-65.

De Freitas, J., H. Wintz, et al. (2003). "Yeast, a model organism for iron and copper metabolism studies." BioMetals 16(1): 185-197.

Decottignies, A., A. M. Grant, et al. (1998). "ATPase and Multidrug Transport Activities of the Overexpressed Yeast ABC Protein Yor1p." Journal of Biological Chemistry 273(20): 12612-12622.

Decottignies, A., M. Kolaczkowski, et al. (1994). "Solubilization and characterization of the overexpressed PDR5 multidrug resistance nucleotide triphosphatase of yeast." Journal of Biological Chemistry 269(17): 12797-12803.

Delahodde, A., T. Delaveau, et al. (1995). "Positive autoregulation of the yeast transcription factor Pdr3p, which is involved in control of drug resistance." Molecular and Cellular Biology 15(8): 4043-4051. 
Delahodde, A., R. Pandjaitan, et al. (2001). "Pse1/Kap121-dependent nuclear localization of the major yeast multidrug resistance (MDR) transcription factor Pdr1." Molecular Microbiology 39(2): 304-313.

Delaunay, A., D. Pflieger, et al. (2002). "A Thiol Peroxidase Is an H2O2 Receptor and Redox-Transducer in Gene Activation." Cell 111(4): 471-481.

Delaveau, T., A. Delahodde, et al. (1994). "PDR3, a new yeast regulatory gene, is homologous to PDR1 and controls the multidrug resistance phenomenon." Molecular and General Genetics MGG 244(5): 501-511.

DeRisi, J., B. van den Hazel, et al. (2000). "Genome microarray analysis of transcriptional activation in multidrug resistance yeast mutants." FEBS Letters 470(2): 156-160.

Devaux, F., E. Carvajal, et al. (2002). "Genome-wide studies on the nuclear PDR3-controlled response to mitochondrial dysfunction in yeast." FEBS Letters 515(1-3): 25-28.

Dickson, R. C. and R. L. Lester (2002). "Sphingolipid functions in Saccharomyces cerevisiae." Biochimica et Biophysica Acta (BBA) - Molecular and Cell Biology of Lipids 1583(1): 13-25.

Egner, R., Y. Mahe, et al. (1995). "Endocytosis and vacuolar degradation of the plasma membrane-localized Pdr5 ATP-binding cassette multidrug transporter in Saccharomyces cerevisiae." Molecular and Cellular Biology 15(11): 5879-5887.

Eisenman, H. C. and E. A. Craig (2004). "Activation of pleiotropic drug resistance by the J-protein and Hsp70-related proteins, Zuo1 and Ssz1." Molecular Microbiology 53(1): 335-344.

Emter, R., A. Heese-Peck, et al. (2002). "ERG6 and PDR5 regulate small lipophilic drug accumulation in yeast cells via distinct mechanisms." FEBS Letters 521(1-3): 57-61.

Fardeau, V., G. I. Lelandais, et al. (2007). "The Central Role of PDR1 in the Foundation of Yeast Drug Resistance." Journal of Biological Chemistry 282(7): 5063-5074.

Fernandes, L., C. Rodrigues-Pousada, et al. (1997). "Yap, a novel family of eight bZIP proteins in Saccharomyces cerevisiae with distinct biological functions." Molecular and Cellular Biology 17(12): 6982-6993.

Fomenko, D. E., A. Koc, et al. (2011). "Thiol peroxidases mediate specific genome-wide regulation of gene expression in response to hydrogen peroxide." Proceedings of the National Academy of Sciences 108(7): 27292734.

Forsburg, S. L. (2001). "The art and design of genetic screens: yeast." Nat Rev Genet 2(9): 659-668.

Friant, S., R. Lombardi, et al. (2001). "Sphingoid base signaling via Pkh kinases is required for endocytosis in yeast." EMBO J 20(23): 6783-6792.

Furuchi, T., H. Ishikawa, et al. (2001). "Two Nuclear Proteins, Cin5 and Ydr259c, Confer Resistance to Cisplatin in Saccharomyces cerevisiae." Molecular Pharmacology 59(3): 470-474.

Gao, C., L. Wang, et al. (2004). "On the Mechanism of Constitutive Pdr1 Activatormediated PDR5 Transcription in Saccharomyces cerevisiae." Journal of Biological Chemistry 279(41): 42677-42686.

Garcia-Gimeno, M. A. and K. Struhl (2000). "Aca1 and Aca2, ATF/CREB Activators in Saccharomyces cerevisiae, Are Important for Carbon Source 
Utilization but Not the Response to Stress." Molecular and Cellular Biology 20(12): 4340-4349.

Gasch, A. P., P. T. Spellman, et al. (2000). "Genomic Expression Programs in the Response of Yeast Cells to Environmental Changes." Molecular Biology of the Cell 11(12): 4241-4257.

Gavin, A.-C., P. Aloy, et al. (2006). "Proteome survey reveals modularity of the yeast cell machinery." Nature 440(7084): 631-636.

Gbelska, Y., J.-J. Krijger, et al. (2006). "Evolution of gene families: the multidrug resistance transporter genes in five related yeast species." FEMS Yeast Research 6(3): 345-355.

Giaever, G., D. D. Shoemaker, et al. (1999). "Genomic profiling of drug sensitivities via induced haploinsufficiency." Nat Genet 21(3): 278-283.

Golin, J., S. V. Ambudkar, et al. (2003). "Studies with Novel Pdr5p Substrates Demonstrate a Strong Size Dependence for Xenobiotic Efflux." Journal of Biological Chemistry 278(8): 5963-5969.

Gömpel-Klein, P. and M. Brendel (1990). "Allelism of SNQ1 and ATR1, genes of the yeast Saccharomyces cerevisiae required for controlling sensitivity to 4nitroquinoline-N-oxide an aminotriazole." Current Genetics 18(1): 93-96.

Gondré-Lewis, M. C., H. I. Petrache, et al. (2006). "Abnormal sterols in cholesterol-deficiency diseases cause secretory granule malformation and decreased membrane curvature." Journal of Cell Science 119(9): 18761885.

Görner, W., E. Durchschlag, et al. (1998). "Nuclear localization of the C2H2 zinc finger protein Msn2p is regulated by stress and protein kinase A activity." Genes \& Development 12(4): 586-597.

Gottesman, M. M., T. Fojo, et al. (2002). "Multidrug resistance in cancer: role of ATP-dependent transporters." Nat Rev Cancer 2(1): 48-58.

Gruenberg, J. and H. Stenmark (2004). "The biogenesis of multivesicular endosomes." Nat Rev Mol Cell Biol 5(4): 317-323.

Gueldry, O., M. Lazard, et al. (2003). "Ycf1p-dependent Hg(II) detoxification in Saccharomyces cerevisiae." European Journal of Biochemistry 270(11): 2486-2496.

Gullo, V., J. McAlpine, et al. (2006). "Drug discovery from natural products." Journal of Industrial Microbiology \& Biotechnology 33(7): 523-531.

Gulshan, K. and W. S. Moye-Rowley (2007). "Multidrug Resistance in Fungi." Eukaryotic Cell 6(11): 1933-1942.

Gulshan, K., S. A. Rovinsky, et al. (2005). "Oxidant-specific Folding of Yap1p Regulates Both Transcriptional Activation and Nuclear Localization." Journal of Biological Chemistry 280(49): 40524-40533.

Gulshan, K., J. A. Schmidt, et al. (2008). "Evidence for the Bifunctional Nature of Mitochondrial Phosphatidylserine Decarboxylase: Role in Pdr3-Dependent Retrograde Regulation of PDR5 Expression." Molecular and Cellular Biology 28(19): 5851-5864.

Gulshan, K., B. Thommandru, et al. (2012). "Proteolytic Degradation of the Yap1 Transcription Factor Is Regulated by Subcellular Localization and the E3 Ubiquitin Ligase Not4." Journal of Biological Chemistry 287(32): 2679626805.

Hahn, S. and E. T. Young (2011). "Transcriptional Regulation in Saccharomyces cerevisiae: Transcription Factor Regulation and Function, Mechanisms of 
Initiation, and Roles of Activators and Coactivators." Genetics 189(3): 705736.

Halliwell, B. and M. Whiteman (2004). "Measuring reactive species and oxidative damage in vivo and in cell culture: how should you do it and what do the results mean?" British Journal of Pharmacology 142(2): 231-255.

Hallstrom, T. C., D. J. Katzmann, et al. (1998). "Regulation of Transcription Factor Pdr1p Function by an Hsp70 Protein in Saccharomyces cerevisiae." Molecular and Cellular Biology 18(3): 1147-1155.

Hallstrom, T. C., L. Lambert, et al. (2001). "Coordinate Control of Sphingolipid Biosynthesis and Multidrug Resistance in Saccharomyces cerevisiae." Journal of Biological Chemistry 276(26): 23674-23680.

Hallstrom, T. C. and W. S. Moye-Rowley (2000). "Multiple Signals from Dysfunctional Mitochondria Activate the Pleiotropic Drug Resistance Pathway in Saccharomyces cerevisiae." Journal of Biological Chemistry 275(48): 37347-37356.

Hanlon, S. E., J. M. Rizzo, et al. (2011). "The Stress Response Factors Yap6, Cin5, Phd1, and Skn7 Direct Targeting of the Conserved Co-Repressor Tup1-Ssn6 in S. cerevisiae." PLoS ONE 6(4): e19060.

Hannun, Y. A. and L. M. Obeid (2008). "Principles of bioactive lipid signalling: lessons from sphingolipids." Nat Rev Mol Cell Biol 9(2): 139-150.

Harbison, C. T., D. B. Gordon, et al. (2004). "Transcriptional regulatory code of a eukaryotic genome." Nature 431(7004): 99-104.

Harshman, K. D., W. S. Moye-Rowley, et al. (1988). "Transcriptional activation by the SV40 AP-1 recognition element in yeast is mediated by a factor similar to AP-1 that is distinct from GCN4." Cell 53(2): 321-330.

Hellauer, K., B. Akache, et al. (2002). "Zinc Cluster Protein Rdr1p Is a Transcriptional Repressor of thePDR5 Gene Encoding a Multidrug Transporter." Journal of Biological Chemistry 277(20): 17671-17676.

Henriksen, J., A. C. Rowat, et al. (2006). "Universal Behavior of Membranes with Sterols." Biophysical Journal 90(5): 1639-1649.

Henry, K. W., A. Wyce, et al. (2003). "Transcriptional activation via sequential histone H2B ubiquitylation and deubiquitylation, mediated by SAGAassociated Ubp8." Genes \& Development 17(21): 2648-2663.

Hertle, K., E. Haase, et al. (1991). "The SNQ3 gene of Saccharomyces cerevisiae confers hyper-resistance to several functionally unrelated chemicals." Current Genetics Jun19(6): 429-433.

Hillenmeyer, M. E., E. Fung, et al. (2008). "The Chemical Genomic Portrait of Yeast: Uncovering a Phenotype for All Genes." Science 320(5874): 362365.

Hnisz, D., M. Tscherner, et al. (2011). "Targeting chromatin in fungal pathogens as a novel therapeutic strategy: histone modification gets infectious." Epigenomics 3(2): 129-132.

Hryciw, T. H., M. T. Tang, et al. (2002). "MMS1; protects against replicationdependent DNA damage in Saccharomyces cerevisiae." Molecular Genetics and Genomics 266(5): 848-857.

Hsuchen, C. C. and D. S. Feingold (1974). "Two types of resistance to polyene antibiotics in Candida albicans." Nature 251(5476): 656-659.

Hu, R.-G., J. Sheng, et al. (2005). "The $\mathrm{N}$-end rule pathway as a nitric oxide sensor controlling the levels of multiple regulators." Nature 437(7061): 981 986. 
Huang, J., H. Zhu, et al. (2004). "Finding new components of the target of rapamycin (TOR) signaling network through chemical genetics and proteome chips." Proceedings of the National Academy of Sciences of the United States of America 101(47): 16594-16599.

Hwang, W. W., S. Venkatasubrahmanyam, et al. (2003). "A Conserved RING Finger Protein Required for Histone H2B Monoubiquitination and Cell Size Control." Molecular Cell 11(1): 261-266.

Ikner, A. and K. Shiozaki (2005). "Yeast signaling pathways in the oxidative stress response." Mutation Research/Fundamental and Molecular Mechanisms of Mutagenesis 569(1-2): 13-27.

Inoue, Y., T. Matsuda, et al. (1999). "Genetic Analysis of Glutathione Peroxidase in Oxidative Stress Response of Saccharomyces cerevisiae." Journal of Biological Chemistry 274(38): 27002-27009.

Isoyama, T., A. Murayama, et al. (2001). "Nuclear Import of the Yeast AP-1-like Transcription Factor Yap1p Is Mediated by Transport Receptor Pse1p, and This Import Step Is Not Affected by Oxidative Stress." Journal of Biological Chemistry 276(24): 21863-21869.

Jamieson, D. J. (1998). "Oxidative stress responses of the yeast Saccharomyces cerevisiae." Yeast 14(16): 1511-1527.

Janke, C., M. M. Magiera, et al. (2004). "A versatile toolbox for PCR-based tagging of yeast genes: new fluorescent proteins, more markers and promoter substitution cassettes." Yeast 21(11): 947-962.

Jia, L., M. Dienhart, et al. (2003). "Yeast Oxa1 interacts with mitochondrial ribosomes: the importance of the C-terminal region of Oxa1." EMBO J 22(24): 6438-6447.

Jia, X.-M., Z.-P. Ma, et al. (2008). "RTA2, a novel gene involved in azole resistance in Candida albicans." Biochemical and Biophysical Research Communications 373(4): 631-636.

Jonikas, M. C., S. R. Collins, et al. (2009). "Comprehensive Characterization of Genes Required for Protein Folding in the Endoplasmic Reticulum." Science 323(5922): 1693-1697.

Jorgensen, P., B. Nelson, et al. (2002). "High-Resolution Genetic Mapping With Ordered Arrays of Saccharomyces cerevisiae Deletion Mutants." Genetics 162(3): 1091-1099.

Jungwirth, $H$. and K. Kuchler (2006). "Yeast ABC transporters - A tale of sex, stress, drugs and aging." FEBS Letters 580(4): 1131-1138.

Kanafani, Z. A. and J. R. Perfect (2008). "Resistance to Antifungal Agents: Mechanisms and Clinical Impact." Clinical Infectious Diseases 46(1): 120128.

Kanazawa, S., M. Driscoll, et al. (1988). "ATR1, a Saccharomyces cerevisiae gene encoding a transmembrane protein required for aminotriazole resistance." Molecular and Cellular Biology 8(2): 664-673.

Katzmann, D. J., T. C. Hallstrom, et al. (1996). "Multiple Pdr1p/Pdr3p Binding Sites Are Essential for Normal Expression of the ATP Binding Cassette Transporter Protein-encoding Gene PDR5." Journal of Biological Chemistry 271(38): 23049-23054.

Katzmann, D. J., T. C. Hallstrom, et al. (1995). "Expression of an ATP-binding cassette transporter-encoding gene (YOR1) is required for oligomycin resistance in Saccharomyces cerevisiae." Molecular and Cellular Biology 15(12): 6875-6883. 
Kavallaris, M. (2010). "Microtubules and resistance to tubulin-binding agents." Nat Rev Cancer 10(3): 194-204.

Kean, L. S., A. M. Grant, et al. (1997). "Plasma Membrane Translocation of Fluorescent-labeled Phosphatidylethanolamine Is Controlled by Transcription Regulators, PDR1 and PDR3." The Journal of Cell Biology 138(2): 255-270.

Kellner, W. A., E. Ramos, et al. (2012). "Genome-wide phosphoacetylation of histone $\mathrm{H} 3$ at Drosophila enhancers and promoters." Genome Research 22(6): 1081-1088.

Kihara, A. and Y. Igarashi (2002). "Identification and Characterization of aSaccharomyces cerevisiae Gene, RSB1, Involved in Sphingoid Longchain Base Release." Journal of Biological Chemistry 277(33): 3004830054.

Kihara, A. and Y. Igarashi (2004). "Cross Talk between Sphingolipids and Glycerophospholipids in the Establishment of Plasma Membrane Asymmetry." Molecular Biology of the Cell 15(11): 4949-4959.

Kitamura, K., M. Taki, et al. (2011). "Fission yeast Ubr1 ubiquitin ligase influences the oxidative stress response via degradation of active Pap1 bZIP transcription factor in the nucleus." Molecular Microbiology 80(3): 739-755.

Kolaczkowska, A. and A. Goffeau (1999). "Regulation of pleiotropic drug resistance in yeast." Drug Resistance Updates 2(6): 403-414.

Kolaczkowska, A., M. Kolaczkowski, et al. (2008). "Compensatory activation of the multidrug transporters Pdr5p, Snq2p, and Yor1p by Pdr1p in Saccharomyces cerevisiae." FEBS Letters 582(6): 977-983.

Kowaltowski, A. J., A. E. Vercesi, et al. (2000). "Catalases and thioredoxin peroxidase protect Saccharomyces cerevisiae against $\mathrm{Ca2+-induced}$ mitochondrial membrane permeabilization and cell death." FEBS Letters 473(2): 177-182.

Krems, B., C. Charizanis, et al. (1996). "The response regulator-like protein Pos9/Skn7 ofSaccharomyces cerevisiae is involved in oxidative stress resistance." Current Genetics 29(4): 327-334.

Kuchler, K. and C. Schüller (2007). ABC Transporters in Yeast - Drug Resistance and Stress Response in a Nutshell. Yeast as a Tool in Cancer Research. J. Nitiss and J. Heitman, Springer Netherlands: 289-314.

Kuge, S., M. Arita, et al. (2001). "Regulation of the Yeast Yap1p Nuclear Export Signal Is Mediated by Redox Signal-Induced Reversible Disulfide Bond Formation." Molecular and Cellular Biology 21(18): 6139-6150.

Kuge, S., N. Jones, et al. (1997). "Regulation of yAP-1 nuclear localization in response to oxidative stress." EMBO J 16(7): 1710-1720.

Kurimoto, K. and M. Saitou (2010). "Single-cell cDNA microarray profiling of complex biological processes of differentiation." Current Opinion in Genetics \&amp; Development 20(5): 470-477.

Kwok, T. C. Y., N. Ricker, et al. (2006). "A small-molecule screen in C. elegans yields a new calcium channel antagonist." Nature 441(7089): 91-95.

LaFayette, S. L., C. Collins, et al. (2010). "PKC Signaling Regulates Drug Resistance of the Fungal Pathogen Candida albicans via Circuitry Comprised of Mkc1, Calcineurin, and Hsp90." PLoS Pathog 6(8): e1001069.

Larochelle, M., S. Drouin, et al. (2006). "Oxidative Stress-Activated Zinc Cluster Protein Stb5 Has Dual Activator/Repressor Functions Required for Pentose 
Phosphate Pathway Regulation and NADPH Production." Molecular and Cellular Biology 26(17): 6690-6701.

Lazard, M., N.-T. Ha-Duong, et al. (2011). "Selenodiglutathione uptake by the Saccharomyces cerevisiae vacuolar ATP-binding cassette transporter Ycf1p." FEBS Journal 278(21): 4112-4121.

Le Crom, S., F. Devaux, et al. (2002). "New Insights into the Pleiotropic Drug Resistance Network from Genome-Wide Characterization of the YRR1 Transcription Factor Regulation System." Molecular and Cellular Biology 22(8): 2642-2649.

Lee, K. K., M. E. Sardiu, et al. (2011). "Combinatorial depletion analysis to assemble the network architecture of the SAGA and ADA chromatin remodeling complexes." Mol Syst Biol 7.

Lee, T. I., N. J. Rinaldi, et al. (2002). "Transcriptional Regulatory Networks in Saccharomyces cerevisiae." Science 298(5594): 799-804.

Li, Z.-S., Y.-P. Lu, et al. (1997). "A new pathway for vacuolar cadmium sequestration in Saccharomyces cerevisiae: YCF1-catalyzed transport of bis(glutathionato)cadmium." Proceedings of the National Academy of Sciences 94(1): 42-47.

Li, Z.-S., M. Szczypka, et al. (1996). "The Yeast Cadmium Factor Protein (YCF1) Is a Vacuolar Glutathione S-Conjugate Pump." Journal of Biological Chemistry 271(11): 6509-6517.

Lin, Y.-y., Y. Qi, et al. (2008). "A comprehensive synthetic genetic interaction network governing yeast histone acetylation and deacetylation." Genes \& Development 22(15): 2062-2074.

Liu, R. and F. J. Sharom (1998). "Proximity of the Nucleotide Binding Domains of the P-glycoprotein Multidrug Transporter to the Membrane Surface: A Resonance Energy Transfer Study." Biochemistry 37(18): 6503-6512.

Liu, Z. (2011). "Molecular mechanisms of yeast tolerance and in situ detoxification of lignocellulose hydrolysates." Applied Microbiology and Biotechnology 90(3): 809-825.

Lobo, I. (2008). "Epistasis: Gene interaction and the phenotypic expression of complex diseases like Alzheimer's." Nature Education 1(1).

Lopez-Rubio, J. J., L. Riviere, et al. (2007). "Shared epigenetic mechanisms control virulence factors in protozoan parasites." Current Opinion in Microbiology 10(6): 560-568.

Lucau-Danila, A., T. Delaveau, et al. (2003). "Competitive Promoter Occupancy by Two Yeast Paralogous Transcription Factors Controlling the Multidrug Resistance Phenomenon." Journal of Biological Chemistry 278(52): 5264152650.

Lum, P. Y., C. D. Armour, et al. (2004). "Discovering Modes of Action for Therapeutic Compounds Using a Genome-Wide Screen of Yeast Heterozygotes." Cell 116(1): 121-137.

MacPherson, S., M. Larochelle, et al. (2006). "A Fungal Family of Transcriptional Regulators: the Zinc Cluster Proteins." Microbiology and Molecular Biology Reviews 70(3): 583-604.

Mahé, Y., Y. Lemoine, et al. (1996). "The ATP Binding Cassette Transporters Pdr5 and Snq2 of Saccharomyces cerevisiae Can Mediate Transport of Steroids in Vivo." Journal of Biological Chemistry 271(41): 25167-25172. 
Mamnun, Y. M., R. Pandjaitan, et al. (2002). "The yeast zinc finger regulators Pdr1p and Pdr3p control pleiotropic drug resistance (PDR) as homo- and heterodimers in vivo." Molecular Microbiology 46(5): 1429-1440.

Mamnun, Y. M., C. Schuller, et al. (2004). "Expression regulation of the yeast PDR5 ATP-binding cassette (ABC) transporter suggests a role in cellular detoxification during the exponential growth phase." FEBS Letters 559(1-3): 111-117.

Manoli, I., S. Alesci, et al. (2007). "Mitochondria as key components of the stress response." Trends in Endocrinology \&amp; Metabolism 18(5): 190-198.

Martens, J. A., J. Genereaux, et al. (1996). "Transcriptional Activation by Yeast PDR1p Is Inhibited by Its Association with NGG1p/ADA3p." Journal of Biological Chemistry 271(27): 15884-15890.

Martínez-Pastor, M., G. Marchler, et al. (1996). "The Saccharomyces cerevisiae zinc finger proteins Msn2p and Msn4p are required for transcriptional induction through the stress response element (STRE)." EMBO J May1;15(9): 2227-2235.

Mathew, B. P. and M. Nath (2009). "Recent Approaches to Antifungal Therapy for Invasive Mycoses." ChemMedChem 4(3): 310-323.

Miyahara, K., D. Hirata, et al. (1996). "yAP-1- and yAP-2-mediated, heat shockinduced transcriptional activation of the multidrug resistance $A B C$ transporter genes in Saccharomyces cerevisiae." Current Genetics 29(2): 103-105.

Miyamoto, Y., K. Machida, et al. (2002). "Identification of Saccharomyces cerevisiae Isoleucyl-tRNA Synthetase as a Target of the G1-specific Inhibitor Reveromycin A." Journal of Biological Chemistry 277(32): 2881028814.

Moraes, G., P. C. Norhcote, et al. (2004). "Structure of the major triterpene glycoside from the sea cucumber Stichopus mollis and evidence to reclassify this species into the new genus Australostichopus." Biochemical Systematics and Ecology 32(7): 637-650.

Morrissey, J. P. and A. E. Osbourn (1999). "Fungal Resistance to Plant Antibiotics as a Mechanism of Pathogenesis." Microbiology and Molecular Biology Reviews 63(3): 708-724.

Morschhäuser, J., K. S. Barker, et al. (2007). "The Transcription Factor Mrr1p Controls Expression of the MDR1 Efflux Pump and Mediates Multidrug Resistance in Candida albicans." PLoS Pathog 3(11): e164.

Mosammaparast, N., Y. Guo, et al. (2002). "Pathways Mediating the Nuclear Import of Histones H3 and H4 in Yeast." Journal of Biological Chemistry 277(1): 862-868.

Moye-Rowley, S. (2003). "Transcriptional control of multidrug resistance in the yeast Saccharomyces" Progress in Nucleic Acid Research \& Molecular Biology73: 251-279.

Moye-Rowley, W. S. (2005). "Retrograde regulation of multidrug resistance in Saccharomyces cerevisiae." Gene 354(0): 15-21.

Moye-Rowley, W. S., K. D. Harshman, et al. (1989). "Yeast YAP1 encodes a novel form of the jun family of transcriptional activator proteins." Genes \& Development 3(3): 283-292.

Munn, A. L. (2001). "Molecular requirements for the internalisation step of endocytosis: insights from yeast." Biochimica et Biophysica Acta (BBA) Molecular Basis of Disease 1535(3): 236-257. 
Ni, L., C. Bruce, et al. (2009). "Dynamic and complex transcription factor binding during an inducible response in yeast." Genes \& Development 23(11): 1351-1363.

Niederacher, G., E. Klopf, et al. (2011). "Interplay of Dynamic Transcription and Chromatin Remodeling: Lessons from Yeast." International Journal of Molecular Sciences 12(8): 4758-4769.

Nourani, A., M. Wesolowski-Louvel, et al. (1997). "Multiple-drug-resistance phenomenon in the yeast Saccharomyces cerevisiae: involvement of two hexose transporters." Molecular and Cellular Biology 17(9): 5453-5460.

Nunes, P. A., S. Tenreiro, et al. (2001). "Resistance and Adaptation to Quinidine in Saccharomyces cerevisiae: Role of QDR1 (YIL120w), Encoding a Plasma Membrane Transporter of the Major Facilitator Superfamily Required for Multidrug Resistance." Antimicrobial Agents and Chemotherapy 45(5): 1528-1534.

Ozcan, S. and M. Johnston (1999). "Function and Regulation of Yeast Hexose Transporters." Microbiology and Molecular Biology Reviews 63(3): 554-569.

Pan, X., P. Ye, et al. (2006). "A DNA Integrity Network in the Yeast Saccharomyces cerevisiae." Cell 124(5): 1069-1081.

Paran, Y., M. Ilan, et al. (2007). "High-throughput screening of cellular features using high-resolution light-microscopy; Application for profiling drug effects on cell adhesion." Journal of Structural Biology 158(2): 233-243.

Park, J.-I., C. M. Grant, et al. (2005). "The high-affinity cAMP phosphodiesterase of Saccharomyces cerevisiae is the major determinant of cAMP levels in stationary phase: involvement of different branches of the Ras-cyclic AMP pathway in stress responses." Biochemical and Biophysical Research Communications 327(1): 311-319.

Parsons, A. B., R. L. Brost, et al. (2004). "Integration of chemical-genetic and genetic interaction data links bioactive compounds to cellular target pathways." Nat Biotech 22(1): 62-69.

Parsons, A. B., A. Lopez, et al. (2006). "Exploring the Mode-of-Action of Bioactive Compounds by Chemical-Genetic Profiling in Yeast." Cell 126(3): 611-625.

Paumi, C. M., M. Chuk, et al. (2009). "ABC Transporters in Saccharomyces cerevisiae and Their Interactors: New Technology Advances the Biology of the ABCC (MRP) Subfamily." Microbiology and Molecular Biology Reviews 73(4): 577-593.

Pepperkok, R. and J. Ellenberg (2006). "High-throughput fluorescence microscopy for systems biology." Nat Rev Mol Cell Biol 7(9): 690-696.

Perlstein, E. O., D. M. Ruderfer, et al. (2007). "Genetic basis of individual differences in the response to small-molecule drugs in yeast." Nat Genet 39(4): 496-502.

Piper, P., Y. Mahe, et al. (1998). "The Pdr12 ABC transporter is required for the development of weak organic acid resistance in yeast." EMBO J 17(15): 4257-4265.

Pohl, A., P. F. Devaux, et al. (2005). "Function of prokaryotic and eukaryotic ABC proteins in lipid transport." Biochimica et Biophysica Acta (BBA) - Molecular and Cell Biology of Lipids 1733(1): 29-52.

Pomorski, T., J. C. M. Holthuis, et al. (2004). "Tracking down lipid flippases and their biological functions." Journal of Cell Science 117(6): 805-813.

Rapaport, D., M. Brunner, et al. (1998). "Fzo1p Is a Mitochondrial Outer Membrane Protein Essential for the Biogenesis of Functional Mitochondria 
in Saccharomyces cerevisiae." Journal of Biological Chemistry 273(32): 20150-20155.

Raymond, C. K., C. J. Roberts, et al. (1992). Biogenesis of the Vacuole in Saccharomyces cerevisiae. International Review of Cytology. W. J. Kwang and F. Martin, Academic Press. Volume 139: 59-120.

Richardson, M. D. (2005). "Changing patterns and trends in systemic fungal infections." Journal of Antimicrobial Chemotherapy 56(suppl 1): i5-i11.

Rockwell, N., H. Wolfger, et al. (2009). "ABC Transporter Pdr10 Regulates the Membrane Microenvironment of Pdr12 in Saccharomyces cerevisiae." Journal of Membrane Biology 229(1): 27-52.

Rogers , B., A. Decottignies, et al. (2001). "The pleitropic drug ABC transporters from Saccharomyces cerevisiae" Journal of Molecular Microbiology and Biotechnology Apr 3(2): 207-214.

Ross, S. J., V. J. Findlay, et al. (2000). "Thioredoxin Peroxidase Is Required for the Transcriptional Response to Oxidative Stress in Budding Yeast." Molecular Biology of the Cell 11(8): 2631-2642.

Rowe, L. A., N. Degtyareva, et al. (2012). "Yap1: A DNA damage responder in Saccharomyces cerevisiae." Mechanisms of Ageing and Development 133(4): 147-156.

Rutledge, R. M., L. Esser, et al. (2011). "Toward understanding the mechanism of action of the yeast multidrug resistance transporter Pdr5p: A molecular modeling study." Journal of Structural Biology 173(2): 333-344.

Sá-Correia, I. and S. Tenreiro (2002). "The multidrug resistance transporters of the major facilitator superfamily, 6 years after disclosure of Saccharomyces cerevisiae genome sequence." Journal of Biotechnology 98(2-3): 215-226.

Sass, P., J. Field, et al. (1986). "Cloning and characterization of the high-affinity cAMP phosphodiesterase of Saccharomyces cerevisiae." Proceedings of the National Academy of Sciences 83(24): 9303-9307.

Schjerling, P. and S. Holmberg (1996). "Comparative Amino Acid Sequence Analysis of the C6 Zinc Cluster Family of Transcriptional Regulators." Nucleic Acids Research 24(23): 4599-4607.

Schmitt, A. P. and K. McEntee (1996). "Msn2p, a zinc finger DNA-binding protein, is the transcriptional activator of the multistress response in Saccharomyces cerevisiae." Proceedings of the National Academy of Sciences 93(12): 5777-5782.

Schuldiner, M., S. R. Collins, et al. (2005). "Exploration of the Function and Organization of the Yeast Early Secretory Pathway through an Epistatic Miniarray Profile." Cell 123(3): 507-519.

Schüller, C., Y. M. Mamnun, et al. (2007). "Membrane-active Compounds Activate the Transcription Factors Pdr1 and Pdr3 Connecting Pleiotropic Drug Resistance and Membrane Lipid Homeostasis in Saccharomyces cerevisiae." Molecular Biology of the Cell 18(12): 4932-4944.

Seedorf, M. and P. A. Silver (1997). "Importin/karyopherin protein family members required for mRNA export from the nucleus." Proceedings of the National Academy of Sciences 94(16): 8590-8595.

Servos, J., E. Haase, et al. (1993). "Gene SNQ2 of Saccharomyces cerevislae which confers resistance to 4-nitroquinoline-N-oxide and other chemicals, encodes a $169 \mathrm{kDa}$ protein homologous to ATP-dependent permeases." Molecular and General Genetics MGG 236(2): 214-218. 
Shahi, P., K. Gulshan, et al. (2007). "Negative Transcriptional Regulation of Multidrug Resistance Gene Expression by an Hsp70 Protein." Journal of Biological Chemistry 282(37): 26822-26831.

Shahi, P., K. Gulshan, et al. (2010). "Differential Roles of Transcriptional Mediator Subunits in Regulation of Multidrug Resistance Gene Expression in Saccharomyces cerevisiae." Molecular Biology of the Cell 21 (14): 24692482.

Shahi, P. and W. S. Moye-Rowley (2009). "Coordinate control of lipid composition and drug transport activities is required for normal multidrug resistance in fungi." Biochimica et Biophysica Acta (BBA) - Proteins \&amp; Proteomics 1794(5): 852-859.

Shimazu, M., T. Sekito, et al. (2005). "A Family of Basic Amino Acid Transporters of the Vacuolar Membrane from Saccharomyces cerevisiae." Journal of Biological Chemistry 280(6): 4851-4857.

Sietsma, H., R. J. Veldman, et al. (2001). "The Involvement of Sphingolipids in Multidrug Resistance." Journal of Membrane Biology 181(3): 153-162.

Simon, J. A. and A. Bedalov (2004). "Yeast as a model system for anticancer drug discovery." Nat Rev Cancer 4(6): 481-487.

Simonetti, G., C. Passariello, et al. (2007). "Histone deacetylase inhibitors may reduce pathogenicity and virulence in Candida albicans." FEMS Yeast Research 7(8): 1371-1380.

Sipo, G. and K. Kuchler (2006). "Fungal ATP-Binding Cassette (ABC) Transporters in Drug Resistance \& Detoxification." 7(4): 471-481.

Smith, E. and A. Shilatifard (2010). "The Chromatin Signaling Pathway: Diverse Mechanisms of Recruitment of Histone-Modifying Enzymes and Varied Biological Outcomes." Molecular Cell 40(5): 689-701.

Šubik, J., S. Ulaszewski, et al. (1986). "Genetic mapping of nuclear mucidin resistance mutations in \&lt;i\&gt;Saccharomyces cerevisiae\&lt;/i\&gt." Current Genetics 10(9): 665-670.

Sun, Y., R. Taniguchi, et al. (2000). "Sli2 (Ypk1), a Homologue of Mammalian Protein Kinase SGK, Is a Downstream Kinase in the Sphingolipid-Mediated Signaling Pathway of Yeast." Molecular and Cellular Biology 20(12): 44114419.

Sydorskyy, Y., D. J. Dilworth, et al. (2003). "Intersection of the Kap123p-Mediated Nuclear Import and Ribosome Export Pathways." Molecular and Cellular Biology 23(6): 2042-2054.

Tanaka, T., S. Izawa, et al. (2005). "GPX2, Encoding a Phospholipid Hydroperoxide Glutathione Peroxidase Homologue, Codes for an Atypical 2-Cys Peroxiredoxin in Saccharomyces cerevisiae." Journal of Biological Chemistry 280(51): 42078-42087.

te Welscher, Y. M., L. Jones, et al. (2010). "Natamycin Inhibits Vacuole Fusion at the Priming Phase via a Specific Interaction with Ergosterol." Antimicrobial Agents and Chemotherapy 54(6): 2618-2625.

te Welscher, Y. M. t., H. H. t. Napel, et al. (2008). "Natamycin Blocks Fungal Growth by Binding Specifically to Ergosterol without Permeabilizing the Membrane." Journal of Biological Chemistry 283(10): 6393-6401.

Tenreiro, S., P. C. Rosa, et al. (2000). "Expression of the AZR1 gene (ORF YGR224w), encoding a plasma membrane transporter of the major facilitator superfamily, is required for adaptation to acetic acid and 
resistance to azoles in Saccharomyces cerevisiae." Yeast 16(16): 14691481.

Tenreiro, S., R. C. Vargas, et al. (2005). "The yeast multidrug transporter Qdr3 (Ybr043c): localization and role as a determinant of resistance to quinidine, barban, cisplatin, and bleomycin." Biochemical and Biophysical Research Communications 327(3): 952-959.

Thakur, J. K., H. Arthanari, et al. (2008). "A nuclear receptor-like pathway regulating multidrug resistance in fungi." Nature 452(7187): 604-609.

Thevissen, K., B. P. A. Cammue, et al. (2000). "A gene encoding a sphingolipid biosynthesis enzyme determines the sensitivity of Saccharomyces cerevisiae to an antifungal plant defensin from dahlia (Dahlia merckii)." Proceedings of the National Academy of Sciences 97(17): 9531-9536.

Toda, T., M. Shimanuki, et al. (1991). "Fission yeast genes that confer resistance to staurosporine encode an AP-1-like transcription factor and a protein kinase related to the mammalian ERK1/MAP2 and budding yeast FUS3 and KSS1 kinases." Genes \& Development 5(1): 60-73.

Tong, A. H. Y., M. Evangelista, et al. (2001). "Systematic Genetic Analysis with Ordered Arrays of Yeast Deletion Mutants." Science 294(5550): 2364-2368.

Tong, A. H. Y., M. Evangelista, et al. (2001). "Systematic Genetic Analysis with Ordered Arrays of Yeast Deletion Mutants." Science 294(5550): 2364-2368.

Toth, M., I. M. Boros, et al. (2012). "Elevated level of lysine 9-acetylated histone $\mathrm{H} 3$ at the MDR1 promoter in multidrug-resistant cells." Cancer Science 103(4): 659-669.

Ueta, R., A. Fukunaka, et al. (2003). "Pse1p Mediates the Nuclear Import of the Iron-responsive Transcription Factor Aft1p in Saccharomyces cerevisiae." Journal of Biological Chemistry 278(50): 50120-50127.

Valle, V. G. R., M. M. Fagian, et al. (1993). "The Participation of Reactive Oxygen Species and Protein Thiols in the Mechanism of Mitochondrial Inner Membrane Permeabilization by Calcium plus Prooxidants." Archives of Biochemistry and Biophysics 307(1): 1-7.

van den Hazel, H. B., H. Pichler, et al. (1999). "PDR16 and PDR17, Two Homologous Genes ofSaccharomyces cerevisiae, Affect Lipid Biosynthesis and Resistance to Multiple Drugs." Journal of Biological Chemistry 274(4): 1934-1941.

van Heusden, G. P. H., T. J. Wenzel, et al. (1992). "Characterization of the yeast $\mathrm{BMH} 1$ gene encoding a putative protein homologous to mammalian protein kinase II activators and protein kinase $C$ inhibitors." FEBS Letters 302(2): 145-150.

Veal, E. A., S. J. Ross, et al. (2003). "Ybp1 Is Required for the Hydrogen Peroxide-induced Oxidation of the Yap1 Transcription Factor." Journal of Biological Chemistry 278(33): 30896-30904.

Venters, B. J. and B. F. Pugh (2009). "A canonical promoter organization of the transcription machinery and its regulators in the Saccharomyces genome." Genome Research 19(3): 360-371.

Vida, T. A. and S. D. Emr (1995). "A new vital stain for visualizing vacuolar membrane dynamics and endocytosis in yeast." The Journal of Cell Biology 128(5): 779-792.

Vizeacoumar, F. J., N. van Dyk, et al. (2010). "Integrating high-throughput genetic interaction mapping and high-content screening to explore yeast spindle morphogenesis." The Journal of Cell Biology 188(1): 69-81. 
Wachtler, V. and M. K. Balasubramanian (2006). "Yeast lipid rafts? - An emerging view." Trends in Cell Biology 16(1): 1-4.

Wacker, S. A., B. R. Houghtaling, et al. (2012). "Using transcriptome sequencing to identify mechanisms of drug action and resistance." Nat Chem Biol 8(3): 235-237.

Wehrschütz-Sigl, E., H. Jungwirth, et al. (2004). "The transporters Pdr5p and Snq2p mediate diazaborine resistance and are under the control of the gain-of-function allele PDR1-12" Eurepean Journal of Biochemistry 271(6): 1145-1152.

Wemmie, J. A., S. M. Steggerda, et al. (1997). "The Saccharomyces cerevisiae AP-1 Protein Discriminates between Oxidative Stress Elicited by the Oxidants H2O2 and Diamide." Journal of Biological Chemistry 272(12): 7908-7914.

Wendler, F., H. Bergler, et al. (1997). "Diazaborine Resistance in the Yeast Saccharomyces cerevisiae Reveals a Link between YAP1 and the Pleiotropic Drug Resistance Genes PDR1 andPDR3." Journal of Biological Chemistry 272(43): 27091-27098.

Winzeler, E. A., D. D. Shoemaker, et al. (1999). "Functional Characterization of the S. cerevisiae Genome by Gene Deletion and Parallel Analysis." Science 285(5429): 901-906.

Wolfger, H., Y. Mahe', et al. (1997). "The yeast ATP binding cassette (ABC) protein genes PDR10 and PDR15 are novel targets for the Pdr1 and Pdr3 transcriptional regulators." FEBS Letters 418(3): 269-274.

Wolfger, H., Y. M. Mamnun, et al. (2001). "Fungal ABC proteins: pleiotropic drug resistance, stress response and cellular detoxification." Research in Microbiology 152(3-4): 375-389.

Wolfger, H., Y. M. Mamnun, et al. (2004). "The Yeast Pdr15p ATP-binding Cassette $(A B C)$ Protein Is a General Stress Response Factor Implicated in Cellular Detoxification." Journal of Biological Chemistry 279(12): 1159311599.

Wollert, T. and J. H. Hurley (2010). "Molecular mechanism of multivesicular body biogenesis by ESCRT complexes." Nature 464(7290): 864-869.

Woods, R. A., M. Bard, et al. (1974). "Resistance to Polyene Antibiotics and Correlated Sterol Changes in Two Isolates of Candida tropicalis from a Patient with an Amphotericin B-resistant Funguria." Journal of Infectious Diseases 129(1): 53-58.

Workman, C. T., H. C. Mak, et al. (2006). "A Systems Approach to Mapping DNA Damage Response Pathways." Science 312(5776): 1054-1059.

Wu, A., J. A. Wemmie, et al. (1993). "Yeast bZip proteins mediate pleiotropic drug and metal resistance." Journal of Biological Chemistry 268(25): 1885018858.

Wurtele, H., S. Tsao, et al. (2010). "Modulation of histone H3 lysine 56 acetylation as an antifungal therapeutic strategy." Nat Med 16(7): 774-780.

Xia, X., J. Yang, et al. (2010). "Image-Based Chemical Screening Identifies Drug Efflux Inhibitors in Lung Cancer Cells." Cancer Research 70(19): 7723-7733.

Yan, C., L. H. Lee, et al. (1998). "Crm1p mediates regulated nuclear export of a yeast AP-1-like transcription factor." EMBO J 17(24): 7416-7429. 
Yibmantasiri, P., D. C. Leahy, et al. (2012). "Molecular basis for fungicidal action of neothyonidioside, a triterpene glycoside from the sea cucumber, Australostichopus mollis." Molecular BioSystems 8(3): 902-912.

Zemanová, L., A. Schenk, et al. (2003). "Confocal optics microscopy for biochemical and cellular high-throughput screening." Drug Discovery Today 8(23): 1085-1093.

Zhang, X., Z. Cui, et al. (2001). "Cross-talk between Transcriptional Regulators of Multidrug Resistance in Saccharomyces cerevisiae." Journal of Biological Chemistry 276(12): 8812-8819.

Zhang, X., A. Kolaczkowska, et al. (2005). "Transcriptional Regulation by Lge1p Requires a Function Independent of Its Role in Histone H2B Ubiquitination." Journal of Biological Chemistry 280(4): 2759-2770.

Zhao, R., M. Davey, et al. (2005). "Navigating the Chaperone Network: An Integrative Map of Physical and Genetic Interactions Mediated by the Hsp90 Chaperone." Cell 120(5): 715-727.

Zheng, J., J. J. Benschop, et al. (2010). "Epistatic relationships reveal the functional organization of yeast transcription factors." Mol Syst Biol 6. 


\section{Appendix I}

Table A-1:List of gene deletions which cause insignificant change in PDR reporters upon atorvastatin treatment

\begin{tabular}{lll}
\hline $\begin{array}{l}\text { Systematic } \\
\text { Name }\end{array}$ & $\begin{array}{l}\text { Standard } \\
\text { Name }\end{array}$ & Description \\
\hline YDR374C & Putative protein of unknown function \\
YFR018C & Putative protein of unknown function \\
YIL152W & Putative protein of unknown function \\
YNL315C & ATP11 & $\begin{array}{l}\text { Molecular chaperone, required for the assembly of } \\
\text { alpha and beta subunits into the F1 sector of } \\
\text { mitochondrial F1F0 ATP synthase }\end{array}$
\end{tabular}

YER155C BEM2 Rho GTPase activating protein (RhoGAP) involved in the control of cytoskeleton organization and cellular morphogenesis; required for bud emergence

YER177W BMH1 14-3-3 protein, major isoform; controls proteome at post-transcriptional level, binds proteins and DNA, involved in regulation of many processes including exocytosis, vesicle transport, Ras/MAPK signaling, and rapamycin-sensitive signalling

YOR061W CKA2 Alpha' catalytic subunit of casein kinase 2 (CK2), a Ser/Thr protein kinase with roles in cell growth and proliferation; CK2, comprised of CKA1, CKA2, CKB1 and CKB2, has many substrates including transcription factors and all RNA polymerase

YDL155W CLB3 B-type cyclin involved in cell cycle progression; activates Cdc28p to promote the G2/M transition; may be involved in DNA replication and spindle assembly; accumulates during $S$ phase and G2, then targeted for ubiquitin-mediated degradation

YCR017C CWH43 Putative sensor/transporter protein involved in cell wall biogenesis; contains 14-16 transmembrane segments and several putative glycosylation and phosphorylation sites; null mutation is synthetically lethal with pkc1 deletion

YGL078C DBP3 Putative ATP-dependent RNA helicase of the DEADbox family involved in ribosomal biogenesis

YDR294C DPL1 Dihydrosphingosine phosphate lyase, regulates intracellular levels of sphingolipid long-chain base phosphates (LCBPS), degrades phosphorylated long 


\section{YGR071C ENV11 Protein proposed to be involved in vacuolar functions; mutant shows defect in CPY processing and fragmented vacuoles; deletion mutant has increased glycogen accumulation and displays elongated buds; green fluorescent protein (GFP)-fusion protein localizes to the nucleus \\ YGL054C ERV14 Protein localized to COPII-coated vesicles, involved in vesicle formation and incorporation of specific secretory cargo; required for the delivery of bud-site selection protein $A x \mid 2 p$ to cell surface; related to Drosophila cornichon}

YIL065C FIS1 Protein involved in mitochondrial membrane fission and peroxisome abundance; required for localization of Dnm1p and Mdv1p during mitochondrial division; mediates ethanol-induced apoptosis and ethanolinduced mitochondrial fragmentation

YIL098C FMC1 Mitochondrial matrix protein, required for assembly or stability at high temperature of the F1 sector of mitochondrial F1F0 ATP synthase; null mutant temperature sensitive growth on glycerol is suppressed by multicopy expression of Odc1p

YHR176W FMO1 Flavin-containing monooxygenase, localized to the cytoplasmic face of the ER membrane; catalyzes oxidation of biological thiols to maintain the ER redox buffer ratio for correct folding of disulfide-bonded proteins

YGR252W GCN5 Acetyltransferase, modifies N-terminal lysines on histones H2B and H3; acetylates Rsc4p, a subunit of the RSC chromatin-remodeling complex, altering replication stress tolerance; catalytic subunit of the ADA and SAGA histone acetyltransferase complexes; founding member of the Gcn5p-related Nacetyltransferase superfamily; mutant displays reduced transcription elongation in the G-less-based run-on (GLRO) assay

GET1 Subunit of the GET complex; involved in insertion of YGL020C (MDM39) proteins into the ER membrane; required for the retrieval of HDEL proteins from the Golgi to the ER in an ERD2 dependent fashion and for normal mitochondrial morphology and inheritance 
YGR163W GTR2 Putative GTP binding protein that negatively regulates Ran/Tc4 GTPase cycle; activates transcription; subunit of EGO and GSE complexes; required for sorting of Gap1p; localizes to cytoplasm and to chromatin; homolog of human RagC and RagD

YJR147W HMS2 Protein with similarity to heat shock transcription factors; overexpression suppresses the pseudohyphal filamentation defect of a diploid mep1 mep2 homozygous null mutant

YFR038W IRC5 Putative ATPase containing the DEAD/H helicaserelated sequence motif; null mutant displays increased levels of spontaneous Rad52p foci

YER110C KAP123 Karyopherin beta, mediates nuclear import of ribosomal proteins prior to assembly into ribosomes and import of histones $\mathrm{H} 3$ and $\mathrm{H} 4$; localizes to the nuclear pore, nucleus, and cytoplasm; exhibits genetic interactions with RAl1

YKL168C KKQ8 Putative serine/threonine protein kinase with unknown cellular role

YDR532C KRE28 Subunit of a kinetochore-microtubule binding complex with Spc105p that bridges centromeric heterochromatin and kinetochore MAPs and motors, and is also required for sister chromatid bi-orientation and kinetochore binding of SAC components

YCL061C MRC1 S-phase checkpoint protein required for DNA replication; interacts with and stabilizes Pol2p at stalled replication forks during stress, where it forms a pausing complex with Tof1p and is phosphorylated by Mec1p; protects uncapped telomeres

YDR192C NUP42 Subunit of the nuclear pore complex (NPC) that localizes exclusively to the cytoplasmic side; involved in RNA export, most likely at a terminal step; interacts with Gle1p

YJR073C OPI3 Phospholipid methyltransferase (methylene-fatty-acylphospholipid synthase), catalyzes the last two steps in phosphatidylcholine biosynthesis

YGL013C PDR1 Zinc cluster protein that is a master regulator involved in recruiting other zinc cluster proteins to pleiotropic drug response elements (PDREs) to fine tune the regulation of multidrug resistance genes 
YBL005W PDR3 Transcriptional activator of the pleiotropic drug resistance network, regulates expression of ATPbinding cassette $(A B C)$ transporters through binding to cis-acting sites known as PDREs (PDR responsive elements); post-translationally upregulated in cells lacking a functional mitochondrial genome

YGL023C PIB2

Protein binding phosphatidylinositol 3-phosphate, involved in telomere-proximal repression of gene expression; similar to Fab1 and Vps27

YBL051C PIN4 Protein involved in G2/M phase progression and response to DNA damage, interacts with Rad53p; contains an RNA recognition motif, a nuclear localization signal, and several SQ/TQ cluster domains; hyperphosphorylated in response to DNA damage

YHR075C PPE1 Protein with carboxyl methyl esterase activity that may have a role in demethylation of the phosphoprotein phosphatase catalytic subunit; also identified as a small subunit mitochondrial ribosomal protein

YNL169C PSD1 Phosphatidylserine decarboxylase of the mitochondrial inner membrane, converts phosphatidylserine to phosphatidylethanolamine

YAL017W PSK1 One of two (see also PSK2) PAS domain containing $\mathrm{S} / \mathrm{T}$ protein kinases; coordinately regulates protein synthesis and carbohydrate metabolism and storage in response to a unknown metabolite that reflects nutritional status

YJL204C RCY1 F-box protein involved in recycling plasma membrane proteins internalized by endocytosis; localized to sites of polarized growth

YKR055W RHO4 Non-essential small GTPase of the Rho/Rac subfamily of Ras-like proteins, likely to be involved in the establishment of cell polarity

YDR279W RNH202 Ribonuclease H2 subunit, required for RNase H2 activity; related to human AGS2 that causes AicardiGoutieres syndrome

YBL093C ROX3 Subunit of the RNA polymerase II mediator complex; associates with core polymerase subunits to form the RNA polymerase II holoenzyme 
YHR200W RPN10 Non-ATPase base subunit of the 19S regulatory particle (RP) of the $26 \mathrm{~S}$ proteasome; $\mathrm{N}$-terminus plays a role in maintaining the structural integrity of the RP; binds selectively to polyubiquitin chains; homolog of the mammalian S5a protein

YJR127C RSF2 Zinc-finger protein involved in transcriptional control of both nuclear and mitochondrial genes, many of which specify products required for glycerol-based growth, respiration, and other functions

YOL067C RTG1 Transcription factor (bHLH) involved in interorganelle communication between mitochondria, peroxisomes, and nucleus

YGR143W SKN1 Protein involved in sphingolipid biosynthesis; type II membrane protein with similarity to Kre6p

YHR206W SKN7 Nuclear response regulator and transcription factor; physically interacts with the Tup1-Cyc8 complex and recruits Tup1p to its targets; part of a branched twocomponent signaling system; required for optimal induction of heat-shock genes in response to oxidative stress; involved in osmoregulation

YGL115W SNF4 Activating gamma subunit of the AMP-activated Snf1p kinase complex (contains Snf1p and a Sip1p/Sip2p/Gal83p family member); activates glucose-repressed genes, represses glucose-induced genes; role in sporulation, and peroxisome biogenesis

YER161C SPT2 Protein involved in negative regulation of transcription; required for RNA polyadenylation; exhibits regulated interactions with both histones and SWI-SNF components, has similarity to mammalian HMG1 proteins

YBR081C SPT7 Subunit of the SAGA transcriptional regulatory complex, involved in proper assembly of the complex; also present as a C-terminally truncated form in the SLIK/SALSA transcriptional regulatory complex

YCR081W SRB8 Subunit of the RNA polymerase II mediator complex; associates with core polymerase subunits to form the RNA polymerase II holoenzyme; essential for transcriptional

regulation; involved in glucose repression

YDR443C SSN2 Subunit of the RNA polymerase II mediator complex; associates with core polymerase subunits to form the RNA polymerase II holoenzyme; required for stable 
association of Srb10p-Srb11p kinase; essential for transcriptional regulation

YHR064C SSZ1 Hsp70 protein that interacts with Zuo1p (a DnaJ homolog) to form a ribosome-associated complex that binds the ribosome via the Zuo1p subunit; also involved in pleiotropic drug resistance via sequential activation of PDR1 and PDR5; binds ATP

YOR027W STI1 Hsp90 cochaperone, interacts with the Ssa group of the cytosolic Hsp70 chaperones and activates Ssa1p ATPase activity; interacts with Hsp90 chaperones and inhibits their ATPase activity; homolog of mammalian Hop

YER111C SWI4 DNA binding component of the SBF complex (Swi4pSwi6p), a transcriptional activator that in concert with MBF (Mbp1-Swi6p) regulates late G1-specific transcription of targets including cyclins and genes required for DNA synthesis and repair

YBR165W UBS1 Ubiquitin-conjugating enzyme suppressor that functions as a general positive regulator of Cdc34p activity; nuclear protein that may represent a link between nucleocytoplasmic transport and ubiquitin ligase activity

YDR207C UME6 Key transcriptional regulator of early meiotic genes, binds URS1 upstream regulatory sequence, couples metabolic responses to nutritional cues with initiation and progression of meiosis, forms complex with Ime1p, and also with Sin3p-Rpd3p

YPR024W YME1 Catalytic subunit of the mitochondrial inner membrane i-AAA protease complex, which is responsible for degradation of unfolded or misfolded mitochondrial gene products; mutation causes an elevated rate of mitochondrial turnover 


\section{Table A-2:Hit genes from Yap1p-GFP screen with $\mathrm{H}_{2} \mathrm{O}_{2}$ treatment \\ Systematic Standard Description name Name \\ YHR210C \\ YLR345W \\ Putative protein of unknown function; non- essential gene; highly expressed under anaeorbic conditions; sequence similarity to aldose 1-epimerases such as GAL10 \\ Similar to 6-phosphofructo-2-kinase/fructose- 2,6-bisphosphatase enzymes responsible for the metabolism of fructoso-2,6-bisphosphate; mRNA expression is repressed by the Rfx1p-Tup1p- Ssn6p repressor complex; YLR345W is not an essential gene}

YDR448W* ADA2 Transcription coactivator, component of the ADA and SAGA transcriptional adaptor/HAT (histone acetyltransferase) complexes

YHR018C ARG4 Argininosuccinate lyase, catalyzes the final step in the arginine biosynthesis pathway

YGR124W ASN2 Asparagine synthetase, isozyme of Asn1p; catalyzes the synthesis of L-asparagine from Laspartate in the asparagine biosynthetic pathway

YLR412W BER1 Protein involved in microtubule-related processes, N-acetylation; GFP-fusion protein localizes to the cytoplasm and is induced in response to the DNA-damaging agent MMS; YLR412W is not an essential gene; similar to Arabidopsis SRR1 gene

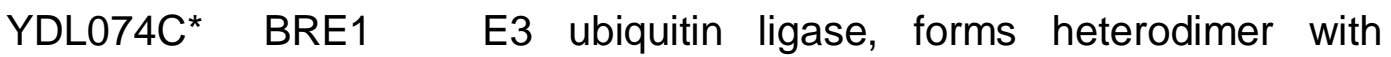
Rad6p to monoubiquinate histone H2B-K123, which is required for the subsequent methylation of histone $\mathrm{H} 3-\mathrm{K} 4$ and $\mathrm{H} 3-\mathrm{K} 79$; required for DSBR, transcription, silencing, and checkpoint control

YCR063W* BUD31 Component of the SF3b subcomplex of the U2 snRNP; diploid mutants display a random budding pattern instead of the wild-type bipolar pattern

YML102W* CAC2 Subunit of chromatin assembly factor I (CAF-1), with RIf2p and Msi1p; chromatin assembly by CAF-1 is important for multiple processes including silencing at telomeres, mating type 
loci, and rDNA; maintenance of kinetochore structure; deactivation of the DNA damage checkpoint after DNA repair; and chromatin dynamics during transcription

YML042W CAT2 Carnitine acetyl-CoA transferase present in both mitochondria and peroxisomes, transfers activated acetyl groups to carnitine to form acetylcarnitine which can be shuttled across membranes

YLR418C* CDC73 Component of the Paf1p complex; binds to and modulates the activity of RNA polymerases I and II; required for expression of certain genes, modification of some histones, and telomere maintenance; involved in transcription elongation as demonstrated by the G-less-based run-on (GLRO) assay

YGL029W* CGR1 Protein involved in nucleolar integrity and processing of the pre-rRNA for the 60S ribosome subunit; transcript is induced in response to cytotoxic stress but not genotoxic stress

YKL137W CMC1 Evolutionarily conserved copper-binding protein of the mitochondrial intermembrane space, may be involved in delivering copper from the matrix to the cytochrome $c$ oxidase complex; contains a twin CX9C motif

YIL036W $^{\star}$ CST6 Basic leucine zipper (bZIP) transcription factor, in ATF/CREB family; mediates transcriptional activation of NCE103 (encoding carbonic anhydrase) in response to low $\mathrm{CO} 2$ levels such as in the ambient air; proposed to be a regulator of oleate responsive genes; involved in utilization of non-optimal carbon sources and chromosome stability

YLR394W CST9 SUMO E3 ligase; required for synaptonemal complex formation; localizes to synapsis initiation sites on meiotic chromosomes; potential Cdc28p substrate

YLR286C CTS1 Endochitinase, required for cell separation after mitosis; transcriptional activation during the G1 phase of the cell cycle is mediated by transcription factor Ace2p 
YDL117W* CYK3

YCL016C DCC1 Subunit of a complex with Ctf8p and Ctf18p that shares some components with Replication Factor $\mathrm{C}$, required for sister chromatid cohesion and telomere length maintenance

YDR121W* DPB4 Shared subunit of DNA polymerase (II) epsilon and of ISW2/yCHRAC chromatin accessibility complex; involved in both chromosomal DNA replication and in inheritance of telomeric silencing

YMR246W FAA4 Long chain fatty acyl-CoA synthetase, activates imported fatty acids with a preference for $\mathrm{C} 12: 0$ C16:0 chain lengths; functions in long chain fatty acid import; important for survival during stationary phase; localized to lipid particles

YAR050W FLO1 Lectin-like protein involved in flocculation, cell wall protein that binds to mannose chains on the surface of other cells, confers floc-forming ability that is chymotrypsin sensitive and heat resistant; similar to Flo5p

YDR070C FMP16 Putative protein of unknown function; proposed to be involved in responding to conditions of stress; the authentic, non-tagged protein is detected in highly purified mitochondria in highthroughput studies

YKR039W GAP1 General amino acid permease; Gap1p senses the presence of amino acid substrates to regulate localization to the plasma membrane when needed

YER083C GET2 Subunit of the GET complex; involved in insertion of proteins into the ER membrane; required for the retrieval of HDEL proteins from the Golgi to the ER in an ERD2 dependent fashion and for meiotic nuclear division

YIR037W* HYR1 Thiol peroxidase that functions as a (GPX3) hydroperoxide receptor to sense intracellular hydroperoxide levels and transduce a redox signal to the Yap1p transcription factor 
YKL161C KDX1 Protein kinase implicated in the Slt2p mitogenactivated (MAP) kinase signaling pathway; interacts with numerous components in the mating pheromone and CWI MAPK pathways; associates with $\mathrm{R} / \mathrm{m} 1 \mathrm{p}$

YIR034C LYS1 Saccharopine dehydrogenase (NAD+, L-lysineforming), catalyzes the conversion of saccharopine to L-lysine, which is the final step in the lysine biosynthesis pathway; also has mRNA binding activity

YDR144C MKC7 GPI-anchored aspartyl protease, member of the yapsin family of proteases involved in cell wall growth and maintenance; shares functions with Yap3p and Kex2p

YPR164W MMS1 Subunit of an E3 ubiquitin ligase complex involved in resolving replication intermediates or preventing the damage caused by blocked replication forks; regulates Ty1 transposition; involved with Rtt101p in nonfunctional rRNA decay

YJL066C MPM1 Mitochondrial membrane protein of unknown function, contains no hydrophobic stretches

YGL136C* MRM2 Mitochondrial 2' O-ribose methyltransferase, required for methylation of $\mathrm{U}(2791)$ in $21 \mathrm{~S}$ rRNA; MRM2 deletion confers thermosensitive respiration and loss of mitochondrial DNA; has similarity to Spb1p and Trm7p, and to E. coli FtsJ/RrmJ

YPL184C* MRN1 RNA-binding protein proposed to be involved in translational regulation; binds specific categories of mRNAs, including those that contain upstream open reading frames (uORFs) and internal ribosome entry sites (IRES)

YOR360W PDE2 High-affinity cyclic AMP phosphodiesterase, component of the cAMP-dependent protein kinase signaling system, protects the cell from extracellular cAMP, contains readthrough motif surrounding termination codon

YPL198W RPL7B Protein component of the large (60S) ribosomal subunit, nearly identical to Rpl7Ap and has similarity to E. coli L30 and rat L7 ribosomal proteins; contains a conserved C-terminal 
Nucleic acid Binding Domain (NDB2)

YFL036W RPO41 Mitochondrial RNA polymerase; single subunit enzyme similar to those of T3 and T7 bacteriophages; requires a specificity subunit encoded by MTF1 for promoter recognition; binding of Mtf1p to the Rpo41p-promoter complex stabilizes the binding and induces DNA bending

YLR287C- RPS30A Protein component of the small (40S) ribosomal A subunit; nearly identical to Rps30Bp and has similarity to rat $\mathrm{S} 30$ ribosomal protein

YLR221C* RSA3 Protein with a likely role in ribosomal maturation, required for accumulation of wild-type levels of large (60S) ribosomal subunits; binds to the helicase Dbp6p in pre-60S ribosomal particles in the nucleolus

YCR009C RVS161 Amphiphysin-like lipid raft protein; interacts with Rvs167p and regulates polarization of the actin cytoskeleton, endocytosis, cell polarity, cell fusion and viability following starvation or osmotic stress

YDR159W SAC3 Nuclear pore-associated protein; required for biogenesis of the small ribosomal subunit; component of TREX-2 complex (Sac3p-Thp1pSus1p-Cdc31p) involved in transcription elongation and mRNA export from the nucleus; involved in post-transcriptional tethering of active genes to the nuclear periphery and to nonnascent mRNP; mutant displays reduced transcription elongation in the G-less-based runon (GLRO) assay

YMR127C* SAS2 Histone acetyltransferase (HAT) catalytic subunit of the SAS complex (Sas2p-Sas4p-Sas5p), which acetylates free histones and nucleosomes and regulates transcriptional silencing; member of the MYSTacetyltransferase family

YKL130C SHE2 RNA-binding protein that binds specific mRNAs and interacts with She3p; part of the mRNA localization machinery that restricts accumulation of certain proteins to the bud

YNL236W SIN4 Subunit of the RNA polymerase II mediator complex; associates with core polymerase 
YDL042C SIR2

YDR425W SNX41

YLL021W SPA2 Component of the polarisome, which functions in actin cytoskeletal organization during polarized growth; acts as a scaffold for Mkk1p and Mpk1p cell wall integrity signaling components; potential Cdc28p substrate

YDL159W STE7 Signal transducing MAP kinase kinase involved in pheromone response, where it phosphorylates Fus3p, and in the pseudohyphal/invasive growth pathway, through phosphorylation of Kss1p; phosphorylated by Ste11p, degraded by ubiquitin pathway

YPR009W SUT2 Putative transcription factor; multicopy suppressor of mutations that cause low activity of the cAMP/protein kinase A pathway; highly similar to Sut $1 p$

YAR042W SWH1 Protein similar to mammalian oxysterol-binding protein; contains ankyrin repeats; localizes to the Golgi and the nucleus-vacuole junction

YJL176C SWI3 Subunit of the SWI/SNF chromatin remodeling complex, which regulates transcription by remodeling chromosomes; required for transcription of many genes, including $\mathrm{ADH} 1$, ADH2, GAL1, HO, INO1 and SUC2

YBR223C TDP1 Tyrosyl-DNA Phosphodiesterase I, hydrolyzes 3' and 5'-phosphotyrosyl bonds, involved in the repair of DNA lesions created by topoisomerase $\mathrm{I}$ and topoisomerase $\mathrm{II}$; mutations in human homolog result in the neurodegenerative disease SCANI 
YGL026C TRP5 Tryptophan synthase, catalyzes the last step of tryptophan biosynthesis; regulated by the general control system of amino acid biosynthesis

YOR115C TRS33 One of 10 subunits of the transport protein particle (TRAPP) complex of the cis-Golgi which mediates vesicle docking and fusion; involved in endoplasmic reticulum (ER) to Golgi membrane traffic

YMR022W UBC7 Ubiquitin conjugating enzyme, involved in the ER-associated protein degradation pathway; requires Cue $1 \mathrm{p}$ for recruitment to the ER membrane; proposed to be involved in chromatin assembly

YGR184C UBR1 E3 ubiquitin ligase ( $N$-recognin), forms heterodimer with Rad6p to ubiquitinate substrates in the $\mathrm{N}$-end rule pathway; regulates peptide transport via Cup9p ubiquitination; mutation in human UBR1 causes JohanssonBlizzard Syndrome (JBS)

YPL139W YAR1 Cytoplasmic ankyrin-repeat containing protein of unknown function, proposed to link the processes of $40 \mathrm{~S}$ ribosomal subunit biogenesis and adaptation to osmotic and oxidative stress; expression repressed by heat shock

*gene deletions that prevent Yap1p_GFP to localise to nucleus upon $\mathrm{H}_{2} \mathrm{O}_{2}$ treatment. The rest of the list were genes which $50 \%$ of cells population Yap1p_GFP localized to nucleus upon $\mathrm{H} 2 \mathrm{O} 2$ treatment and $50 \%$ of the population did not. 
Table A-3 :Hit genes from Yap1p-GFP screen with diethyl maleate treatment

\begin{tabular}{lll}
\hline $\begin{array}{l}\text { Systematic } \\
\text { Name }\end{array}$ & $\begin{array}{l}\text { Standard } \\
\text { Name }\end{array}$ & Description \\
\hline YBR184W & $\begin{array}{l}\text { Putative protein of unknown function; YBR184W is } \\
\text { not an essential gene }\end{array}$ \\
YBR238C & $\begin{array}{l}\text { Mitochondrial membrane protein with similarity to } \\
\text { Rmd9p; not required for respiratory growth but } \\
\text { causes a synthetic respiratory defect in } \\
\text { combination with rmd9 mutations; transcriptionally } \\
\text { upregulated by TOR; deletion increases life span }\end{array}$
\end{tabular}

YBR285W

Putative protein of unknown function; YBR285W is not an essential gene

YDL012C

Tail-anchored plasma membrane protein containing a conserved CYSTM module, possibly involved in response to stress; may contribute to non-homologous end-joining (NHEJ) based on ydl012c htz1 double null phenotype

YCR011C ADP1 Putative ATP-dependent permease of the ABC transporter family of proteins

YCR010C ADY2 Acetate transporter required for normal sporulation; phosphorylated in mitochondria

YBR262C AIM5 Mitochondrial inner membrane protein; subunit of the mitochondrial inner membrane organizing system (MitOS, MICOS, or MINOS), a scaffold-like structure on the intermembrane space side of the inner membrane which has a role in the maintenance of crista junctions and inner membrane architecture

YJL115W ASF1 Nucleosome assembly factor, involved in chromatin assembly and disassembly, antisilencing protein that causes derepression of silent loci when overexpressed; plays a role in regulating Ty1 transposition

YBR270C BIT2 Subunit of TORC2, a membrane-associated complex that regulates actin cytoskeletal dynamics during polarized growth and cell wall integrity; interacts with SIm1p and SIm2p, homologous $\mathrm{PH}$ domain-containing TORC2 substrates; similar to Bit61p

YCR034W FEN1 Fatty acid elongase, involved in sphingolipid 
biosynthesis; acts on fatty acids of up to 24 carbons in length; mutations have regulatory effects on 1,3-beta-glucan synthase, vacuolar ATPase, and the secretory pathway

YLL029W FRA1 Protein involved in negative regulation of transcription of iron regulon; forms an iron independent complex with Fra2p, Grx3p, and Grx4p; cytosolic; mutant fails to repress transcription of iron regulon and is defective in spore formation

YCL036W GFD2 Protein of unknown function, identified as a highcopy suppressor of a dbp5 mutation

YDR358W GGA1 Golgi-localized protein with homology to gammaadaptin, interacts with and regulates Arf1p and Arf2p in a GTP-dependent manner in order to facilitate traffic through the late Golgi

YDL035C GPR1 Plasma membrane $G$ protein coupled receptor (GPCR) that interacts with the heterotrimeric $G$ protein alpha subunit, Gpa2p, and with Plc1p; sensor that integrates nutritional signals with the modulation of cell fate via PKA and cAMP synthesis

YBR244W GPX2 Phospholipid hydroperoxide glutathione peroxidase induced by glucose starvation that protects cells from phospholipid hydroperoxides and nonphospholipid peroxides during oxidative stress

YGL084C GUP1 Plasma membrane protein involved in remodeling GPI anchors; member of the MBOAT family of putative membrane-bound O-acyltransferases; proposed to be involved in glycerol transport

YDL223C HBT1 Substrate of the Hub1p ubiquitin-like protein that localizes to the shmoo tip (mating projection); mutants are defective for mating projection formation, thereby implicating Hbt1p in polarized cell morphogenesis

YBR245C ISW1 Member of the imitation-switch (ISWI) class of ATP-dependent chromatin remodeling complexes; ATPase that forms a complex with loc2p and loc4p to regulate transcription elongation, and a complex with loc3p to repress transcription initiation 
YER110C KAP123 Karyopherin beta, mediates nuclear import of ribosomal proteins prior to assembly into ribosomes and import of histones $\mathrm{H} 3$ and $\mathrm{H} 4$; localizes to the nuclear pore, nucleus, and cytoplasm; exhibits genetic interactions with RAl1

YPR070W MED1 Subunit of the RNA polymerase II mediator complex; associates with core polymerase subunits to form the RNA polymerase II holoenzyme; essential for transcriptional regulation

YCL044C MGR1 Subunit of the mitochondrial (mt) i-AAA protease supercomplex, which degrades misfolded mitochondrial proteins; forms a subcomplex with Mgr3p that binds to substrates to facilitate proteolysis; required for growth of cells lacking mtDNA

YEL007W MIT1 Transcriptional regulator of pseudohyphal growth; protein with sequence similarity to $S$. pombe gti $1+$ (gluconate transport inducer 1) and C. albicans Wor1

YPR164W MMS1 Subunit of an E3 ubiquitin ligase complex involved in resolving replication intermediates or preventing the damage caused by blocked replication forks; regulates Ty1 transposition; involved with Rtt101p in nonfunctional rRNA decay

YCL033C MXR2 Methionine-R-sulfoxide reductase, involved in the response to oxidative stress; protects iron-sulfur clusters from oxidative inactivation along with MXR1; involved in the regulation of lifespan

YDL096C OPI6 Dubious open reading frame unlikely to encode a protein, based on available experimental and comparative sequence data; partially overlaps verified gene PMT1/YDL095W; YDL096C is not essential

YDL127W PCL2 Cyclin, interacts with cyclin-dependent kinase Pho85p; member of the Pcl1,2-like subfamily, involved in the regulation of polarized growth and morphogenesis and progression through the cell cycle; localizes to sites of polarized cell growth

YCL056C PEX34 Peroxisomal integral membrane protein that regulates peroxisome populations; interacts with Pex11p, Pex25p, and Pex27p to control both 
constitutive peroxisome division and peroxisome morphology and abundance during peroxisome proliferation

YDR276C PMP3 Small plasma membrane protein related to a family of plant polypeptides that are overexpressed under high salt concentration or low temperature, not essential for viability, deletion causes hyperpolarization of the plasma membrane potential

YDL104C QRI7 Highly conserved mitochondrial protein, essential (petite) for t6A modification of mitochondrial tRNAs that decode ANN codons; similar to Kae1p and E. coli YgjD, both of which are also required for tRNA t6A modification

YGL163C RAD54 DNA-dependent ATPase, stimulates strand exchange by modifying the topology of doublestranded DNA; involved in the recombinational repair of double-strand breaks in DNA during vegetative growth and meiosis; member of the SWI/SNF family

YDR076W RAD55 Protein that stimulates strand exchange by stabilizing the binding of Rad51p to singlestranded DNA; involved in the recombinational repair of double-strand breaks in DNA during vegetative growth and meiosis; forms heterodimer with Rad57p

YAL036C RBG1 Member of the DRG family of GTP-binding proteins; associates with translating ribosomes; interacts with Tma46p, Ygr250cp, Gir2p and Yap1p via two-hybrid

YCR028C- RIM1 Single-stranded DNA-binding protein essential for A (petite) mitochondrial genome maintenance; involved in mitochondrial DNA replication

YBR229C ROT2 Glucosidase II catalytic subunit required for normal cell wall synthesis; mutations in rot2 suppress tor2 mutations, and are synthetically lethal with rot 1 mutations

YLL002W RTT109 Histone acetyltransferase critical for cell survival in the presence of DNA damage during $S$ phase; acetylates $\mathrm{H} 3-\mathrm{K} 56$ and $\mathrm{H} 3-\mathrm{K} 9$; involved in nonhomologous end joining and in regulation of Ty1 transposition; interacts physically with Vps75p 
YBR280C SAF1 F-Box protein involved in proteasome-dependent degradation of Aah1p during entry of cells into quiescence; interacts with Skp1

YBR228W SLX1 Subunit of a complex, with SIx4p, that hydrolyzes 5 ' branches from duplex DNA in response to stalled or converging replication forks; function overlaps with that of Sgs1p-Top3p

YDL013W SLX5 Subunit of the SIx5-SIx8 SUMO-targeted ubiquitin ligase (STUbL) complex, stimulated by SUMOmodified substrates; contains a RING domain and two SIMs (SUMO-interacting motifs); forms SUMO-dependent nuclear foci, including DNA repair centers

YBR289W SNF5 Subunit of the SWI/SNF chromatin remodeling complex involved in transcriptional regulation; functions interdependently in transcriptional activation with Snf2p and Snf6p

YCR081W SRB8 Subunit of the RNA polymerase II mediator complex; associates with core polymerase subunits to form the RNA polymerase II holoenzyme; essential for transcriptional regulation; involved in glucose repression

YDL133W SRF1 Regulator of phospholipase D (Spo14p); interacts with Spo14p and regulates its catalytic activity; capable of buffering the toxicity of C16:0 platelet activating factor, a lipid that accumulates intraneuronally in Alzheimer's patients

YJL092W SRS2 DNA helicase and DNA-dependent ATPase involved in DNA repair, needed for proper timing of commitment to meiotic recombination and transition from Meiosis I to II; blocks trinucleotide repeat expansion; affects genome stability

YDR293C SSD1 Translational repressor with a role in polar growth and wall integrity; regulated by Cbk1p phosphorylation to effect bud-specific translational control and localization of specific mRNAs; interacts with TOR pathway components; contains a functional $\mathrm{N}$-terminal nuclear localization sequence and nucleocytoplasmic shuttling appears to be critical to Ssd1p function

YDR443C SSN2 Subunit of the RNA polymerase II mediator 
complex; associates with core polymerase subunits to form the RNA polymerase II holoenzyme; required for stable association of Srb10p-Srb11p kinase; essential for transcriptional regulation

YCR030C SYP1 Protein of unknown function that is involved in endocytic site formation; may regulate assembly and disassembly of the septin ring; colocalizes and interacts with septin subunits; potential role in actin cytoskeletal organization

YBR240C THI2 Transcriptional activator of thiamine biosynthetic genes; interacts with regulatory factor Thi3p to control expression of thiamine biosynthetic genes with respect to thiamine availability; acts together with Pdc2p to respond to thiaminediphosphate demand, possibly as related to carbon source availability; zinc finger protein of the $\mathrm{Zn}(\mathrm{II}) 2 \mathrm{Cys} 6$ type

YGL096W TOS8 Homeodomain-containing protein and putative transcription factor found associated with chromatin; target of SBF transcription factor; induced during meiosis and under cell-damaging conditions; similar to Cup9p transcription factor

YML028W TSA1 Thioredoxin peroxidase, acts as both a ribosomeassociated and free cytoplasmic antioxidant; selfassociates to form a high-molecular weight chaperone complex under oxidative stress; deletion results in mutator phenotype

YBR216C YBP1 Protein required for oxidation of specific cysteine residues of the transcription factor Yap1p, resulting in the nuclear localization of Yap1p in response to stress

YBR264C YPT10 Rab family GTP-binding protein that contains the PEST signal sequence specific for proteolytic enzymes; may be involved in vesicular transport; overexpression leads to accumulation of Golgi-like cisternae with budding vesicles

YML001W YPT7 Rab family GTPase; GTP-binding protein of the rab family; required for homotypic fusion event in vacuole inheritance, for endosome-endosome fusion, similar to mammalian Rab7 


\section{Appendix II}

Yibmantasiri, P., D. C. Leahy, et al. (2012). "Molecular basis for fungicidal action of neothyonidioside, a triterpene glycoside from the sea cucumber, Australostichopus mollis." Molecular BioSystems 8(3): 902-912. 